Rolf Hagedorn

\title{
Steuerhinterziehung und Finanzpolitik
}




\section{Rolf Hagedorn}

\section{Steuerhinterziehung und Finanzpolitik}

Die Arbeit enthält nach einem Überblick zur ökonomischen Theorie der Steuerhinterziehung eine Anwendung dieser Theorie auf die Hinterziehung von Steuern auf Zinserträge privater Haushalte. Dabei wird auch auf die Wirkungen einer Quellensteuer eingegangen. Zur derzeit praktizierten Besteuerung von Zinserträgen wird eine Alternative skizziert. Die in der Arbeit dargestellten mikroökonomischen Modelle gehen teils von einer einmaligen, teils von periodisch wiederholten Veranlagungen zur Einkommensteuer aus. Im letzten Fall ist die bei der Steuerhinterziehung zu erwartende Strafe von der Vergangenheit des Hinterziehers abhängig.

Rolf Hagedorn wurde 1958 in Rheda, Westfalen, geboren. Er studierte Volkswirtschaftslehre an den Universitäten Köln und Bielefeld. Von 1986 bis 1990 war er Assistent bei Prof. Dr. V. Arnold an der FernUniversität Hagen. Seit 1990 ist er Referent im Bundesministerium der Finanzen, Bonn. Promotion 1991 an der FernUniversität. 
Steuerhinterziehung und Finanzpolitik

Ein theoretischer Beitrag unter besonderer Berücksichtigung der Hinterziehung von Zinsertrăgen 


\section{FINANZWISSENSCHAFTLICHE SCHRIFTEN}

Herausgegeben von den Professoren

Albers, Krause-Junk, Littmann, Oberhauser, Pohmer, Schmidt

Band 44

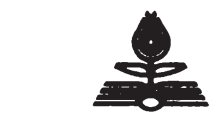

PETER LANG

Frankfurtam Main · Bern - New York · Paris 


\section{Rolf Hagedorn}

\section{Steuerhinterziehung und Finanzpolitik}

Ein theoretischer Beitrag unter besonderer Berücksichtigung der Hinterziehung von Zinserträgen

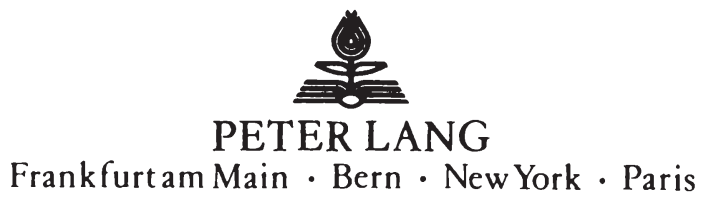


Die Deutsche Bibliothek - CIP-Einheitsaufnahme

Hagedorn, Rolf:

Steuerhinterziehung und Finanzpolitik : ein theoretischer

Beitrag unter besonderer Berūcksichtigung der Hinterziehung von Zinserträgen / Rolf Hagedom. - Frankfurt am Main ; Berm ;

Now York ; Paris : Lang, 1991

(Finanzwissenschaftliche Schriften ; Bd. 44)

Zugl.: Hagen, Fernuniv., Diss., 1991

ISBN 3-631-44228-9

NE: GT

Open Access: The online version of this publication is published on www.peterlang.com and www.econstor.eu under the international Creative Commons License CC-BY 4.0. Learn more on how you can use and share this work: http://creativecommons. org/licenses/by/4.0.

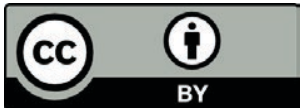

This book is available Open Access thanks to the kind support of ZBW - Leibniz-Informationszentrum Wirtschaft.

\author{
D 708 \\ ISSN 0170-8252 \\ ISBN 3-631-44228-9 \\ ISBN 978-3-631-75230-2 (eBook) \\ (c) Verlag Peter Lang GmbH, Frankfurt am Main 1991 \\ Alle Rechte vorbehalten.
}

Das Werk einschlieBlich aller seiner Teile ist urheberrechtlich geschützt. Jede Verwertung außerhalb der engen Grenzen des Urheberrechtsgesetzes ist ohne Zustimmung des Verlages unzulässig und strafbar. Das gilt insbesondere fūr Vervielfăltigungen, Übersetzungen, Mikroverfilmungen und die Einspeicherung und Verarbeitung in elektronischen Systemen.

Printed in Germany 123467 
Sin impuestos ni el sueño.

(Spanisches Sprichwort)

Alles sollte so einfach wie möglich gemacht werden, aber nicht einfacher.

(Albert Einstein zugeschrieben) 
Rolf Hagedorn - 978-3-631-75230-2

Downloaded from PubFactory at 01/11/2019 06:51:51AM

via free access 


\section{Inhaltsverzeichnis}

Einleitung 1

1 Die ökonomische Theorie der Steuerhinterziehung: Das Grundmodell 5

1.1 Veranlagung und Quellenabzug im Besteuerungsverfahren . . . . . 5

1.2 Das Modell . . . . . . . . . . . . . . . . . 7

1.3 Hinterziehung von Einkommensteuern . . . . . . . . . . 10

1.4 Steuerhinterziehung und Steuervermeidung . . . . . . . . . . . 19

1.5 Die Prämissen des Grundmodells - eine Diskussion . . . . . . . . 23

1.6 Zusammenfassung . . . . . . . . . . . . . 26

2 Erweiterungen des Grundmodells 28

2.1 Variationen des Steuersystems . . . . . . . . . . . . . . . 28

2.1.1 Alternative Geldstrafen . . . . . . . . . . . . . . 28

2.1.2 Variable Entdeckungswahrscheinlichkeit. . . . . . . . . 42

2.1.3 Progressiver Tarif . . . . . . . . . . . . . . . 43

2.2 Endogene Steuerbemessungsgrundlage . . . . . . . . . . 46

2.2.1 Modelle der Steuerhinterziehung mit endogener Steuerbemessungsgrundlage . . . . . . . . . . . . 47

2.2.2 Umsatzsteuerhinterziehung . . . . . . . . . . . . . . . 49

2.2.3 Hinterziehung von Arbeitseinkommen . . . . . . . . . 50

2.3 Interdependenzen zwischen Steuerhinterziehern . . . . . . . . 53

2.3.1 Steuerhinterziehung und Arbeitsmarktgleichgewicht . . . . 54

2.3.2 Steuerhinterziehung und die Bereitstellung öffentlicher Güter 56

2.3.3 Steuerhinterziehung als Epidemie . . . . . . . . . 59

2.3.4 Versicherung der Folgen einer Steuerprüfung . . . . . . . 62

2.4 Zusammenfassung . . . . . . . . . . . . . 63

3 Hinterziehung von Zinseinkommen 65

3.1 Einleitung . . . . . . . . . . . . . . . . 65

3.2 Das Modell . . . . . . . . . . . . . . . . . 69 
3.3 Komparative Statik bei einer inneren Lösung . . . . . . . . . . . 72

3.3.1 Strafen und Kontrollen bei endogenem Zinseinkommen . . . 72

3.3.2 Zinsniveau und die Hinterziehung von Kapitalerträgen . . . 75

3.4 Komparative Statik bei einer Ecklösung . . . . . . . . . . . . 77

3.5 Ergebnisse bei proportionalem Tarif und der Strafe $\Phi_{1} \ldots \ldots \ldots 79$

3.6 Die Strafe nach deutschem Recht . . . . . . . . . . . 81

3.7 Direkt progressiver Tarif . . . . . . . . . . . . . 82

3.8 Zusammenfassung . . . . . . . . . . . . . . 84

4 Die Quellensteuer auf Zinserträge und die Hinterziehung $\begin{array}{ll}\text { von Einkommensteuern } & \mathbf{8 7}\end{array}$

4.1 Einleitung . . . . . . . . . . . . . . . . . . 87

4.2 Das Modell . . . . . . . . . . . . . . . . . . . . . 89

4.3 Die Hinterziehung von Zinseinkommen . . . . . . . . . . . 92

4.4 "The Net Taxation Case" . . . . . . . . . . . . . . . . . 96

4.5 Variable Zinserträge . . . . . . . . . . . . . . . 98

4.6 Die Aufkommenswirkung der Quellensteuer und ihr Beitrag zu einer gleichmäßigeren Besteuerung . . . . . . . . . . . 102

4.7 Progressiver Tarif . . . . . . . . . . . . . . . . 105

4.8 Abschließende Bemerkungen . . . . . . . . . . . 107

5 Steuerhinterziehung und Bankgeheimnis unter einer

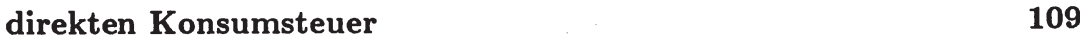

5.1 Einleitung . . . . . . . . . . . . . . . . . 109

5.2 Erfassung der Zinserträge oder direkte Besteuerung des Konsums? . 110

5.3 Konsumsteuer und Bankgeheimnis . . . . . . . . . . 113

5.3.1 Die Konsumsteuer bei vollständiger Information des Fiskus . 113

5.3.2 Hinterziehung von Konsumsteuern und Gleichmäßigkeit der Besteuerung . . . . . . . . . . . . . . 117

5.3.3 Zwei alternative Verfahren des Progressionsausgleichs . . . . 125

5.4 Verzicht auf die Einsichtnahme in Sparkonten? . . . . . . . . . . . 127

5.4.1 Kontrollmitteilungen und Quellenabzug zur Erhebung einer Konsumsteuer . . . . . . . . . . . . . . 127

5.4 .2 Unsicherheit . . . . . . . . . . . . . . . 128

5.4 .3 Auslandsanlagen . . . . . . . . . . . . . 131

5.4.4 Die Administration einer Konsumsteuer . . . . . . . . . 132

5.5 Abschließende Bemerkungen . . . . . . . . . . . . 135

ii 
6 Steuerhinterziehung bei rückwirkenden Kontrollen und einer verschärften Prüfung Verdächtiger: Modelle mit wiederholter Veranlagung

6.1 Einleitung . . . . . . . . . . . . . . . . . 137

6.2 Rückwirkende Kontrollen . . . . . . . . . . . . . . . . . 140

6.2.1 Das Modell mit einer Verjährungsfrist von einem Jahr . . . 142

6.2.2 Das Markov-Entscheidungsproblem des Steuerpflichtigen . . 147

6.2.3 Der Einfluß der Verjährungsfrist auf die Steuerhinterziehung 154

6.2.4 Stochastisches Einkommen . . . . . . . . . . . . . 162

6.2.5 Der Nettoeffekt rückwirkender Kontrollen . . . . . . . . 165

6.3 Verhaltensabhängige Entdeckungswahrscheinlichkeiten . . . . . 169

6.3.1 Ein Modell mit zwei Gruppen: Verdächtige und weniger verdächtige Steuerpflichtige . . . . . . . . . . 170

6.3 .2 Drei Gruppen . . . . . . . . . . . . . . . 173

6.3.3 Gruppenbildung und Steueraufkommen . . . . . . . 175

6.4 Zusammenfassung und Diskussion . . . . . . . . . . . 179

Zusammenfassung: Steuerhinterziehung und Finanzpolitik $\quad 185$

$\begin{array}{ll}\text { Anhang } & 191\end{array}$

Symbolverzeichnis 203

$\begin{array}{ll}\text { Literaturverzeichnis } & 205\end{array}$ 
Rolf Hagedorn - 978-3-631-75230-2

Downloaded from PubFactory at 01/11/2019 06:51:51AM

via free access 


\section{Einleitung}

\section{Abgrenzung}

Diese Arbeit ist eine positive mikroökonomische Partialanalyse der Entscheidungen eines Steuerzahlers, dessen Steuerschuld von seinen Angaben in seiner Steuererklärung abhängt. Sie zeigt, wie ein Steuerpflichtiger, der nicht freiwillig einen Teil seines Einkommens an den Staat abtritt, sondern nur an dem Erwartungsnutzen seines Nettoeinkommens interessiert ist, unter verschiedenen steuerlichen Rahmenbedingungen seine Steuererklärung ausfüllt. Auf Ansätze, die den partialanalytischen Rahmen überschreiten, und normative Fragen - wie sollte der Staat die für einen Steuerhinterzieher relevanten Rahmenbedingungen gestalten ? - wird eingegangen. Sie stehen jedoch nicht im Mittelpunkt der Arbeit.

Die Beiträge dreier wirtschaftswissenschaftlicher Disziplinen, die sich ebenfalls mit der Steuerhinterziehung befassen, werden nicht oder nur am Rande behandelt:

- der Makroökonomie,

- der empirischen Forschung (statistische Analysen und experimentelle Wirtschaftsforschung)

- und der betriebswirtschaftlichen Steuerlehre.

Das heißt aber nicht, daß gesamtwirtschaftliche Implikationen der mikroökonomischen Modelle, Ergebnisse empirischer Arbeiten und steuerrechtliche Details nicht beachtet würden.

In einer umfassenden Untersuchung der Steuerhinterziehung wären die genannten Disziplinen unverzichtbar. Der Anspruch dieser Arbeit ist jedoch bescheidener.

\section{Aufgabenstellung}

Die Ziele dieser Arbeit sind

- die systematische Darstellung der positiven Theorie der Steuerhinterziehung 
- und die Weiterentwicklung dieser Theorie und ihre Anwendung auf die "Hinterziehung von Zinserträgen" privater Haushalte.

Die ersten Modelle zur Analyse der Entscheidungen eines Steuerhinterziehers sind 1972 und 1973 von Allingham/Sandmo bzw. Srinivasan veröffentlicht worden. Seitdem sind eine Vielzahl weiterer Arbeiten erschienen. Übersichtsdarstellungen zur Theorie der Steuerhinterziehung sind rar, in Lehrbücher ist sie noch nicht eingegangen. Es lohnt sich daher, das vorhandene Wissen systematisch aufzuarbeiten.

Innerhalb der positiven Theorie der Steuerhinterziehung gibt es noch viele Lücken. Ich versuche in dieser Arbeit, einige von ihnen zu verkleinern. Gleichzeitig geht es mir um eine Anwendung der Modelle der Steuerhinterziehung auf ein Thema, das in der wirtschaftspolitischen Diskussion der letzten Jahre eine gewisse Rolle gespielt hat: die Hinterziehung von Zinserträgen privater Haushalte vor der Einkommensteuer.

Zu welchen Fragen trägt die Theorie der Steuerhinterziehung Antworten bei? Die drei Fragen, die in dieser Arbeit behandelt werden, sollen hier kurz angegeben werden. Erst soll jedoch auf eine Vorfrage hingewiesen werden, die nicht behandelt wird. Warum Steuern hinterzogen werden, wird per Annahme beantwortet: um Geld zu sparen. Neben Steuerpflichtigen, die von diesem Motiv getrieben werden, gibt es vielleicht auch Steuerzahler, die freiwillig ihren Beitrag zur Finanzierung öffentlicher Leistungen erbringen. Diese stehen jedoch verständlicherweise nicht im Mittelpunkt einer Theorie der Steuerhinterziehung. Die drei Fragen sind:

a) Unter welchen Bedingungen werden Steuern hinterzogen?

b) Führt die Entwicklung zu höheren (Brutto-) Einkommen zu einer größeren oder kleineren Steuerehrlichkeit?

c) Wie beeinflußt der Einsatz finanzpolitischer Instrumente die Steuerhinterziehung, das Steueraufkommen und die Steuergerechtigkeit?

Bei dem letzten, zentralen Punkt ist zunächst an die Steuerpolitik im geläufigen Sinne zu denken: Wie wirken sich eine Änderung des Steuertarifs, die Einführung einer Quellensteuer oder ein Wechsel der Bemessungsgrundlage direkter Steuern aus? Neben diesen Variablen gibt es eine Gruppe finanzpolitischer Parameter, die die Häufigkeit von Steuerprüfungen und die zu erwartenden Strafen für die Steuerhinterziehung betreffen. Diese Parameter sind zentrale Bestandteile der in dieser Arbeit dargestellten Modelle, da diese davon ausgehen, daß niemand freiwillig Steuern zahlt.

Eine Antwort auf die genannten Fragen ist für eine "gerechte und ökonomisch rationale Finanzpolitik" (F. Neumark) unverzichtbar, da sie davon ausgehen muß, 
daß zumindest ein Teil der Steuerpflichtigen versucht, seine Abgabenlast zu verringern, statt die gesetzlich fällige Steuerschuld zu begleichen, und auf diesem Wege die Ziele der Finanzpolitik durchkreuzen kann. Zudem stellt $\S 85$ Abs. 1 der Abgabenordnung ausdrücklich fest, daß "die Finanzbehörden die Steuern nach Maßgabe der Gesetze gleichmäßig festsetzen und zu erheben" haben. "Insbesondere haben sie sicherzustellen, daß Steuern nicht verkürzt ... werden". Den Sinn dieser Vorschrift kann man leicht begründen, wenn man davon ausgeht, daß die Besteuerung die wichtigste Finanzierungsart öffentlicher Leistungen ist und die einzelnen Steuerpflichtigen ein nicht zu ungleiches Gewicht in der "gesellschaftlichen Wohlfahrtsfunktion" haben, so daß Steuerhinterzieher nicht einfach auf Kosten anderer ihren Finanzierungsbeitrag kürzen können. Es stellen sich daher die Fragen, welche Mittel tauglich und welche Mittel am tauglichsten sind, um die Steuerhinterziehung zu begrenzen und zu einer gleichmäßigen Besteuerung beizutragen, ohne mit allzu großen Nebenwirkungen verbunden zu sein.

$\mathrm{Zu}$ diesen Fragen bietet diese Arbeit Antworten an. Nicht beantwortet wird die Frage nach einer optimalen Finanzpolitik im Sinne der Optimalsteuertheorie. Die wohlfahrtsmaximierende Wahl aller (vieler) finanzpolitischer Parameter ist eine formidable Aufgabe, wenn mit der Steuerhinterziehung gerechnet werden muß. Da das Verhalten der Steuerpflichtigen in der Optimalsteuertheorie als Nebenbedingung beachtet werden muß, kann diese Arbeit jedoch als Grundlage für zukünftige Forschung in dieser Richtung dienen.

\section{Aufbau}

Die Arbeit ist in formaler Hinsicht wie folgt aufgebaut: Kapitel 1 enthält das Grundmodell, auf dem alle mikroökonomischen Analysen der Steuerhinterziehung basieren. Wer dieses Kapitel gelesen hat, kann seine Lektüre mit jedem der nächsten fünf Kapitel fortsetzen, ohne Gefahr zu laufen, mehr als einmal zurückblättern zu müssen. Jedes Kapitel ist mit einer Einleitung und Zusammenfassung versehen. Die den einzelnen Kapiteln zugrundeliegenden Modellvarianten werden (samt Notation) stets Schritt für Schritt erläutert. Wer die ganze Arbeit liest, wird Wiederholungen feststellen. Sie sind der Preis dafür, daß die Eintrittsbarrieren zu den einzelnen Kapiteln so niedrig wie möglich gehalten worden sind.

Trotz der formalen Unverbundenheit der Kapitel $2-6$ gibt es inhaltlich einen roten Faden. Die Kapitel 1 und 2 enthalten eine Darstellung des Standes der positiven ökonomischen Theorie der Steuerhinterziehung. Während Kapitel 1 grundlegende Aspekte behandelt, geht es in Kapitel 2 um eine systematische Darstellung der Varianten des Grundmodells, die in der Literatur zu finden sind. In den Kapiteln 3 - 5 wird der Zusammenhang von Steuerhinterziehung und Spar- 
entscheidungen thematisiert. In Kapitel 3 geht es um die Hinterziehung von Zinserträgen privater Haushalte vor der Einkommensteuer, wobei eine Quellensteuer auf Zinserträge nicht berücksichtigt wird. Kapitel 4 behandelt den Einfluß einer Quellensteuer auf Zinserträge auf die Steuerhinterziehung ${ }^{1}$. Kapitel 5 untersucht die Konsequenzen der Steuerhinterziehung, wenn nicht das Einkommen, sondern der Konsum Bemessungsgrundlage der direkten Besteuerung privater Haushalte wäre, und enthält einen Vorschlag zur Administration einer Ausgabensteuer. In Kapitel 6 wird ein Merkmal der Steuererhebung in den Mittelpunkt gestellt, das auch in Kapitel 5 schon eine Rolle spielt: Ein Steuerpflichtiger wird nicht einmal in seinem Leben, sondern immer wieder (jedes Jahr) zur Steuer veranlagt. Dieses Merkmal ist dann von Bedeutung, wenn die Häufigkeit von Steuerprüfungen und die Strafen von dem Verhalten eines Steuerpflichtigen in der Vergangenheit abhängig sind.

\footnotetext{
${ }^{1}$ Kapitel 4 liegt ein Aufsatz zugrunde, der im Finanzarchiv N.F., Band 47, Heft 1 von 1989, erschienen ist.
} 


\section{Kapitel 1}

\section{Die ökonomische Theorie der Steuerhinterziehung: Das Grundmodell}

Zielsetzung der ersten zwei Kapitel ist es, den Stand der positiven ökonomischen Theorie der Steuerhinterziehung darzustellen. Sie sind ein systematischer Überblick, der den "roten Faden" in der Literatur deutlich machen und es ermöglichen soll, die folgenden Kapitel dieser Arbeit einzuordnen. Ein Überblick ist naturgemäß nicht vollständig. Auf einige Aspekte der Theorie der Steuerhinterziehung wird nur verwiesen. Andere werden erst in späteren Kapiteln dieser Arbeit aufgegriffen ${ }^{1}$.

In Kapitel 1 wird das Grundmodell dargestellt, auf dem alle Analysen der Steuerhinterziehung aufbauen. Darüber hinaus enthält es eine Einleitung zu Verfahren der Steuererhebung und die Abgrenzung der Steuerhinterziehung von anderen Aktivitäten, die auf eine Steuerersparnis abzielen. Kapitel 2 enthält einen Überblick zu Erweiterungen des Grundmodells, die die Literatur anbietet.

\subsection{Veranlagung und Quellenabzug im Besteuerungsverfahren}

Um Steuern zu erheben, gibt es für den Fiskus grundsätzlich zwei Möglichkeiten, die mit Veranlagungs- und Quellenabzugsverfahren bezeichnet werden. In dem Veranlagungsverfahren hat der Steuerzahler die Pflicht, dem Finanzamt in einer Steuererklärung alle für die Feststellung der Höhe und der Zusammensetzung

\footnotetext{
${ }^{1}$ Es gibt einen Übersichtsartikel zu der "Economic analysis of tax evasion" (Cowell, 1986). Der gut lesbare Artikel von Cowell ist aber zum Teil etwas ungenau und macht die Systematik und Lücken in der Literatur nicht sehr deutlich.
} 
der Steuerbemessungsgrundlage erheblichen Tatsachen mitzuteilen. Von Dritten erhält die Finanzbehörde zunächst keine Informationen. Auf der Grundlage der Angaben in der Steuererklärung wird die Steuerschuld festgesetzt und von dem Steuerzahler bezahlt. Bei einem reinen Abzugsverfahren muß der Steuerzahler nichts tun. Ein Dritter - der Arbeitgeber oder die Bank zum Beispiel - wird verpflichtet, den Fiskus über alle steuerlich relevanten Tatsachen in Kenntnis zu setzen und die Steuer auf Rechnung des Steuerzahlers abzuführen. Steuerzahler und -pflichtiger müssen also nicht identisch sein (vgl. §33 Abs. 1 AO). Nur bei dem reinen Veranlagungsverfahren ist der Steuerzahler, der mit der Abgabe belastet werden soll, gleichzeitig der einzige zur Zahlung Verpflichtete.

Die zwei Besteuerungsverfahren sind die beiden Eckpunkte einer Reihe von Alternativen, die sich nach dem Grad der Aktivität, zu der der Steuerzahler verpflichtet wird, unterscheiden (vgl. Shoup, 1969, S. 428). In dem reinen Veranlagungsverfahren muß zunächst nur der Steuerzahler dem Finanzamt Angaben machen. Das Abzugsverfahren weist ihm eine völlig passive Rolle zu. Dazwischen liegen Erhebungsformen, bei denen sowohl der Steuerzahler als auch ein Dritter Erklärungen abgeben und Zahlungen leisten müssen.

Die Organisation der Steuererhebung ist für die Frage, ob ein Steuerzahler sich dem Zugriff des Fiskus entziehen kann, von einiger Bedeutung. Ein Steuerzahler hat keine Möglichkeit, Steuern zu hinterziehen, wenn er der oben beschriebenen reinen Form des Abzugsverfahrens unterliegt. Bei dem Veranlagungsverfahren hat er jedoch die Möglichkeit, Steuern zu "verkürzen" und "nicht gerechtfertigte Steuervorteile zu erlangen", indem er "den Finanzbehörden ... über steuerlich erhebliche Tatsachen unrichtige oder unvollständige Angaben macht" oder "die Finanzbehörden pflichtwidrig über steuerlich erhebliche Tatsachen in Unkenntnis läßt ..." (§ $370 \mathrm{AO})^{2}$. Um eine Hinterziehung von Steuern zu begrenzen, ist das Veranlagungsverfahren mit steuerlichen Prüfungen und Strafen verbunden. Aber auch das Abzugsverfahren verzichtet nicht auf Kontrollen und Strafen. Hier wird der zum Steuerabzug verpflichtete Dritte durch eine mögliche Überprüfung seiner Angaben und Strafen für den Fall der Hinterziehung von Abgaben anderer zur Ehrlichkeit angehalten.

Die theoretische Literatur zur Steuerhinterziehung hat sich ausgehend von Allingham/Sandmo (1972) auf die reine Form des Veranlagungsverfahrens konzentriert. Erst in jüngster Zeit ist auch das reine Quellenabzugsverfahren untersucht worden, bei dem ausschließlich ein Dritter Steuerpflichtiger ist (Marelli, 1984). Neu sind dagegen Versuche, Besteuerungsverfahren zu untersuchen, die zwischen den beiden Extremen des reinen Abzugs- und Veranlagungsverfahrens liegen (Ya-

\footnotetext{
${ }^{2}$ Diese Definition der Steuerhinterziehung muß bis Abschnitt 1.4 ausreichen. Erst dann wird die gesetzliche Definition durch eine ökonomische Charakterisierung ergänzt.
} 
niv, 1988, und Kapitel 4 dieser Arbeit) ${ }^{3}$. Sie sind gerade für die Bundesrepublik von besonderem Interesse, da die Einkommensteuer sowohl durch Veranlagung als auch durch Quellenabzug erhoben wird.

Unabhängig von der Form des Besteuerungsverfahrens gilt für jede Steuer: Zumindest eine Wirtschaftseinheit hat die Möglichkeit der Hinterziehung, indem sie den Finanzbehörden falsche Angaben macht oder steuerlich erhebliche Tatsachen verschweigt ${ }^{4}$. Der Fiskus kann nicht bei jeder ökonomischen Transaktion zugegen sein und ist folglich auf die Mitarbeit der Zensiten angewiesen. Da aber viele nicht freiwillig Steuern zahlen, verwendet der Staat Zwangsmittel. Die Wirkungen verschiedener Kontrollen und Strafen stehen daher im Mittelpunkt der Theorie der Steuerhinterziehung.

\subsection{Das Modell}

Dieser Abschnitt stellt das Modell eines individuell-rational handelnden Steuerpflichtigen dar, das allen Analysen der Steuerhinterziehung zugrunde liegt. Die Auswertung des Modells enthält Abschnitt 1.3. Das Modell geht von dem Veranlagungsverfahren aus: Nur von dem Steuerzahler selbst erhält der Fiskus Angaben über die Steuerbemessungsgrundlage, solange er dessen Angaben keiner Überprüfung unterzieht. Ich folge Allingham/Sandmo (1972) und stelle das Modell anhand der Einkommensbesteuerung natürlicher Personen dar. Auf andere mögliche Interpretationen wird unten kurz eingegangen.

Der Haushalt ist verpflichtet, den Finanzbehörden sein Einkommen wahrheitsgemäß zu deklarieren und die dem deklarierten Einkommen entsprechende Steuer zu zahlen: Die Steuerveranlagung ist die "einzigartige Situation", in der "derjenige, der letztlich die 'Zeche' zahlt, auch noch selbst die Rechnung ... schreiben muß” (Bilsdorfer, 1989, S. 17). Wenn der Haushalt nicht sein gesamtes Einkommen angibt, kann er Steuern sparen. Wird er jedoch überprüft und wird festgestellt, daß er Steuern hinterzogen hat, muß er sie nachzahlen. Zusätzlich wird er mit einer Geldstrafe belegt. Gegenüber der Situation, in der der Steuerpflichtige (im folgenden Stpfl. genannt) ehrlich ist, kann er durch die Hinterziehung von Einkommen also entweder Geld sparen oder Geld verlieren: Die Steuerhinterziehung ist eine riskante Aktivität. Das im folgenden dargestellte Modell erfaßt diese Risikosituation eines Steuerhinterziehers mit Hilfe der mikroökonomischen Theorie unter Unsicherheit. Das Modell stammt von Allingham/Sandmo (1972), die verwendete Geldstrafe von Yitzhaki (1974).

\footnotetext{
${ }^{3}$ Verschiedene Zwischenformen beschreibt Shoup (1969, S. 428 - 430).

${ }^{4}$ Das gilt selbst für die Kopfsteuer: Der Steuerzahler kann die Meldung seiner Person unterlassen.
} 
Sei $Y$ das (legal verdiente) zu versteuernde Einkommen, $D$ das deklarierte und $H$ das hinterzogene Einkommen. Definitorisch gilt $H \equiv Y-D$. Es wird eine proportionale Steuer mit dem Satz $t$ angenommen $(0<t<1)$. Nach Erhalt des exogen bestimmten Einkommens am Anfang des betrachteten Zeitabschnitts deklariert der Stpfl. den Betrag $D$, der von $Y$ abweichen kann, wenn zu geringe Einkünfte oder zu hohe Abzüge angegeben werden. Werden seine Angaben von der Finanzbehörde nicht überprüft, ist sein Einkommen nach Steuer gleich

$$
G=Y-t D=Y(1-t)+t H
$$

Über eine mögliche Steuerprüfung werden folgende Annahmen getroffen: Sie führt stets zu einer Aufdeckung des tatsächlichen Einkommens; die Steuerhinterziehung zieht eine Verurteilung nach sich. Es ergeben sich zwei Konsequenzen. Erstens ist die hinterzogene Steuer $t H$ nachzuzahlen. Zweitens wird der Haushalt mit einer Geldstrafe belegt. Für das Grundmodell gehe ich von einer Geldstrafe in Form eines konstanten Vielfachen der hinterzogenen Steuer aus. Die Bemessungsgrundlage dieser Strafe ist einleuchtend: Die hinterzogene Steuer mißt die Schwere des Schadens, der dem Fiskus (ich sage absichtlich nicht der Gesellschaft) entsteht. Wenn die Strafe die Schwere des Vergehens berücksichtigen soll, muß sie von der hinterzogenen Steuer abhängig sein. Die Geldstrafe

$$
S_{1}=\alpha t H, \quad \alpha>0
$$

ist die einfachste Form, diese Abhängigkeit zu formulieren. Der Parameter $\alpha$ wird als Strafsatz bezeichnet ${ }^{5}$. Im Fall einer Prüfung ist das Einkommen nach Steuer und Strafe gleich

$$
\begin{aligned}
P & =Y-t D-(1+\alpha) t H \\
& =Y(1-t)-\alpha t H
\end{aligned}
$$

Das Nettoeinkommen ist nun gleich dem tatsächlichen Einkommen nach Steuer abzüglich der Geldstrafe ${ }^{6}$.

Wovon hängt es ab, ob der Stpfl. geprüft wird? Es gibt viele Gründe für eine steuerliche Prüfung: von eifrigen Beamten, die Kontrollmitteilungen auswerten und Miet- und Verkaufsangebote in Zeitungen studieren, bis hin zu einer Denunziation durch Dritte (siehe Bielsdorfer, 1989, zu den vielfältigen Informationsquellen der Finanzverwaltung). Ein Steuerhinterzieher wird versuchen, so wenig

\footnotetext{
${ }^{5}$ Die hinterzogene Steuer ist in vielen Ländern Ausgangsgröße der Strafe, so z.B. in den USA und der Bundesrepublik.

${ }^{6}$ In der Literatur wird die Steuernachzahlung vielfach unter die Strafe subsumiert. Yitzhaki (1974) z.b. bezeichnet $(1+\alpha) t H$ als Strafe und $1+\alpha$ als Strafsatz. Ich ziehe es vor, die Nachzahlung von der Strafe im strafrechtlichen Sinne zu trennen. Qualitativ andere Ergebnisse ergeben sich dadurch jedoch nicht.
} 
Verdachtsmomente wie möglich zu produzieren. Da er seine Umwelt letztlich jedoch nicht kontrollieren kann, bleibt eine gewisse Unsicherheit, die das Modell in folgende Annahme übersetzt: Der potentielle Hinterzieher ist zu der subjektiven Überzeugung gelangt, seine Steuererklärung werde mit einer Wahrscheinlichkeit $p, 0<p<1$, überprüft. Mit einer Wahrscheinlichkeit $q=1-p$ hat er Glück und entgeht einer Kontrolle. $p$ kann jedoch auch eine objektive Wahrscheinlichkeit sein, wenn die Finanzbehörde zufällig ausgewählte $p \%$ aller Steuererklärungen genauer prüft.

Wenden wir uns den Zielvorstellungen des repräsentativen Stpfl. zu. Seine Präferenzordnung über unsichere Konsummöglichkeiten genüge den Axiomen der Erwartungsnutzentheorie. Der Konsum $C$ sei das einzige Argument seiner von Neumann/Morgenstern-Nutzenfunktion $U(C)$. Für die Ableitungen dieser Funktion gelte $U^{\prime}(C)>0$ und $U^{\prime \prime}(C)<0$ : Der Stpfl. zeichnet sich durch Nichtsättigung und Risikoaversion aus ${ }^{7}$. Er wird das hinterzogene Einkommen so wählen, daß der Erwartungsnutzen $E(U(C))=q U(G)+p U(P)$ aus dem Konsum des Einkommens nach Steuer und Strafe maximal ist.

Das Problem des Stpfl. lautet damit

$$
\begin{array}{cl}
\max _{H} & q U(Y(1-t)+t H)+p U(Y(1-t)-\alpha t H) \\
\text { u.d.N. } & 0 \leqq H \leqq Y
\end{array}
$$

Statt $H$ könnte auch $D$ als Entscheidungsvariable gewählt werden. In (1.1) und (1.2) wäre $H$ dann durch $Y-D$ zu ersetzen. Anstelle von $0 \leqq H \leqq Y$ tritt $Y \geqq D \geqq 0$. Ich ziehe es vor, mit $H$ als Variable zu arbeiten, da sich damit an späterer Stelle Vereinfachungen ergeben.

Die Nebenbedingung $0 \leqq H \leqq Y$ bedarf eines Kommentars (vgl. Baldry, 1984, S. 159). Die Schranke $H \leqq Y$ besagt, daß der Stpfl. kein negatives Einkommen deklarieren kann. Sie ist sinnvoll, wenn der Steuertarif $T(B)$ der Bemessungsgrundlage $B$ keinen Verlustausgleich zuläßt, d.h. wenn (bei proportionalem Tarif) gilt:

$$
T(B)=\left\{\begin{array}{lll}
t B & \text { wenn } & B \geqq 0 \\
0 & \text { wenn } & B<0
\end{array}\right.
$$

Die Schranke $H \geqq 0$ kann entsprechend begründet werden. Ein Stpfl., der mehr als sein tatsächliches Einkommen deklariert $(H<0 \Leftrightarrow D>Y)$, wird dafür vom Fiskus im Fall einer Prüfung keine Prämie (negative Strafe) erhalten. Die Strafe als Funktion von $H$ hat daher dieselbe Struktur wie der Tarif ohne Verlustausgleich. Wenn es sich aber nicht lohnt, zuviel Steuern zu zahlen, wird ein rationaler Stpfl. sich auf $H \geqq 0$ beschränken.

\footnotetext{
${ }^{7}$ Eine kurze Einführung in die Erwartungsnutzentheorie findet sich z.B. in Varian (1984, S. 155 - 162) und Baumol (1972, S. 536 - 552).
} 
Das oben formulierte Optimierungsproblem kann durch

$$
\begin{array}{rl}
\max _{G, P} & q U(G)+p U(P) \\
\text { u.d.N. } & P=(1-t) Y-\alpha(G-(1-t) Y) \\
& (1-t) Y \leqq G \leqq Y
\end{array}
$$

ersetzt werden. (1.3) ergibt sich, indem man (1.1) in (1.2) einsetzt und $H$ eliminiert. (1.4) folgt aus

$$
0 \leqq H=\frac{G-(1-t) Y}{t} \leqq Y
$$

Aus der optimalen Lösung $G^{*}$ und $P^{*}$ und den Beschränkungen (1.1) und (1.2) kann man natürlich $H^{*}$, die Lösung der zuerst angegebenen Optimierungsaufgabe ableiten. Das zweite Problem wird hier angegeben, da es einer hilfreichen graphischen Darstellung zugänglich ist, in der man auch $H^{*}$ wiederfinden kann. Mit einem Stern gekennzeichnete Variablen sind im folgenden stets das Ergebnis einer Optimierung.

Weitere Varianten der Optimierungsaufgabe erhält man, wenn statt $H$ oder $D$ die Größen $t H$ oder $H / Y$ als Entscheidungsvariable verwendet werden. Dies soll hier nicht explizit geschehen. Die Auswertung des Modells wird jedoch die Beziehungen zwischen dem hinterzogen Einkommen, der hinterzogenen Steuer und dem deklarierten Einkommen, die sich bei Parameteränderungen ergeben, betonen.

Bevor wir zu der Auswertung des Grundmodells kommen, sei auf weitere Interpretationen hingewiesen. Bei $Y$ könnte es sich statt um das Einkommen eines einkommensteuerpflichtigen Haushalts auch um den körperschaftsteuerpflichtigen Gewinn eines Unternehmens handeln. $Y$ könnte auch der Wert von Importen sein, der bei Grenzübertritt zu deklarieren ist und einem Wertzoll mit dem Satz $t$ unterliegt. Das Grundmodell ist aufgrund seiner einfachen Struktur offen für verschiedene Deutungen. Erweiterungen des Modells werden diesen Interpretationsspielraum einschränken.

\subsection{Hinterziehung von Einkommensteuern}

Von welchen Faktoren ist die Steuerhinterziehung abhängig? Welcher Art sind diese Abhängigkeiten? Für das Grundmodell sollen diese Fragen im folgenden beantwortet werden. Die Erweiterungen des Grundmodells in den nächsten Kapiteln werden dann zeigen müssen, inwieweit es bei diesen zwei Antworten bleiben kann.

Die Bedingung erster Ordnung für eine innere Lösung $\left(0<H^{*}<Y\right)$ des zuerst formulierten Optimierungsproblems lautet:

$$
\frac{\partial E(U(C))}{\partial H}=E_{H}=q U^{\prime}(G) t-p U^{\prime}(P) t \alpha=0
$$


Die hinreichende Bedingung zweiter Ordnung

$$
E_{H H}=q U^{\prime \prime}(G) t^{2}+p U^{\prime \prime}(P) t^{2} \alpha^{2}<0
$$

ist wegen $U^{\prime \prime}<0$ erfüllt.

Unter welchen Voraussetzungen kommt eine innere Lösung zustande? Für $H^{*}>0 \mathrm{muB}$

$$
\left.E_{H}\right|_{H=0}=U^{\prime}(Y(1-t)) t(q-p \alpha)>0
$$

gelten. Da der Grenznutzen des Konsums und der Steuersatz positiv sind, ist

$$
q-p \alpha>0
$$

eine hinreichende und notwendige Bedingung für einen positiven Umfang des hinterzogenen Einkommens. $q-p \alpha$ ist der erwartete Ertrag pro DM hinterzogener Steuern. Daß dieser Ertrag für eine Wahl $H^{*}>0$ positiv sein muß, kann nicht überraschen. Denn die Risikoaversion impliziert, daß eine Lotterie einem sicheren Einkommen $(1-t) Y$ nur dann vorgezogen wird, wenn ihr Erwartungswert größer als das sichere Einkommen ist. Überraschend ist, daß es nicht von der Höhe der Steuerbelastung abhängt, ob überhaupt hinterzogen wird. In welchem Umfang hinterzogen wird, ist jedoch abhängig von der Besteuerung, wie sich unten noch zeigen wird. Interessant ist noch folgende Beobachtung: Wenn alle Haushalte mit derselben Entdeckungswahrscheinlichkeit kalkulieren und (1.6) erfüllt ist, gibt es den vielzitierten ehrlichen Steuerzahler nicht.

Mit $q-p \alpha \leqq 0$ lohnt sich die Hinterziehung nicht. Werte der Entdeckungswahrscheinlichkeit und des Strafsatzes, die zu

$$
q-p \alpha=1-p(1+\alpha)=0 \quad \Leftrightarrow \quad p=\frac{1}{1+\alpha}
$$

führen, wären gerade ausreichend für eine prohibitive Wirkung der Kontrollen und Strafen. Wenn die Rechtsprechung z.B. einen Wert von $\alpha=1$ vorsieht ${ }^{8}$, errechnet sich eine Kontrollwahrscheinlichkeit von 1/2! Die folgende Analyse, die das Verhalten eines Steuerhinterziehers analysieren will, wird von $q-p \alpha>0$ ausgehen.

Die Bedingung für eine Lösung $H^{*}<Y$ ist

$$
\left.E_{H}\right|_{H=Y}=q U^{\prime}(Y) t-p U^{\prime}(Y(1-t(1+\alpha))) t \alpha<0
$$

Faßt man (1.6) und (1.7) zusammen, ergibt sich

$$
\frac{U^{\prime}(Y)}{U^{\prime}(Y(1-t(1+\alpha)))}<\frac{p \alpha}{q}<1
$$

8 "Für die Bemessung der Hinterziehungsstrafe ... hat sich ein Geldbetrag in Höhe von 50 bis $100 \%$ der verkürzten Steuer eingebürgert” (Kühn/Kutter/Hofmann, 1987, Anm. 12 a) zu $§ 370$ AO, S. 800). 


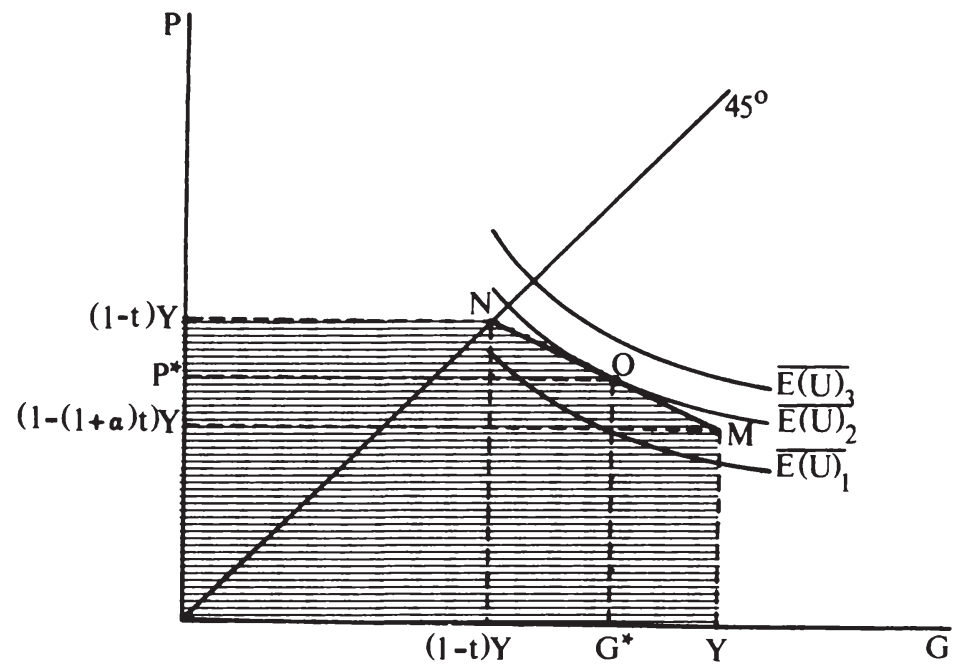

$\mathrm{Da}$ die linke Seite dieser Bedingung kleiner eins ist, gibt es Werte der Parameter $p$ und $\alpha$, die den Stpfl. zu einer Wahl $0<H^{*}<Y$ veranlassen (Allingham/Sandmo, 1972, S. 326). Es kann für gegebene Parameterwerte jedoch auch zu einer Ecklösung kommen. Die Ungleichung zeigt, daß dies einerseits von der Krümmung der Nutzenfunktion, zum anderen von dem erwarteten Ertrag der Steuerhinterziehung abhängt. In folgendem Fall ist die Wahl $H^{*}=Y$ ausgeschlossen: Mit $\alpha=(1-t) / t$ führt sie zu $P=0$. Wenn die Nutzenfunktion die Eigenschaft $U^{\prime}(0)=\infty$ hat, ist (1.7) dann immer erfüllt, da der Stpfl. nicht riskieren wird, bei einer Entdeckung ohne ein positives Nettoeinkommen zu bleiben.

Die Wahl des Stpfl. kann graphisch dargestellt werden. In Abbildung 1.1 ist die Budgetbeschränkung (1.3) als Gerade $N M$ eingezeichnet. Die Eckpunkte $N$ und $M$ sind durch (1.4) festgelegt. Die Steigung von $N M$ ist $d P / d G=-\alpha$. In $N$ ist der Stpfl. ehrlich und hat unabhängig von der Steuerprüfung ein verfügbares Einkommen $(1-t) Y$. In $M$ hinterzieht er sein gesamtes Einkommen. Die hinterzogene Steuer $t Y$ ist gleich dem horizontalen Abstand von $M$ zu $N$. Die maximale Strafe $\alpha t Y$ ist gleich dem vertikalen Abstand $N M$. Der Haushalt kann jede Kombination von $P$ und $G$ auf der schraffierten Fläche erreichen. Da sein Nutzen mit dem Konsum zunimmt, wird er sich auf die Gerade $N M$ beschränken. Der Punkt $N$ kann wegen $q-p \alpha>0$ ausgeschlossen werden. Das läßt sich wie folgt zeigen. Die Präferenzen des Stpfl. bezüglich $P$ und $G$ lassen sich durch ein 
Abbildung 1.2: Die Ecklösung

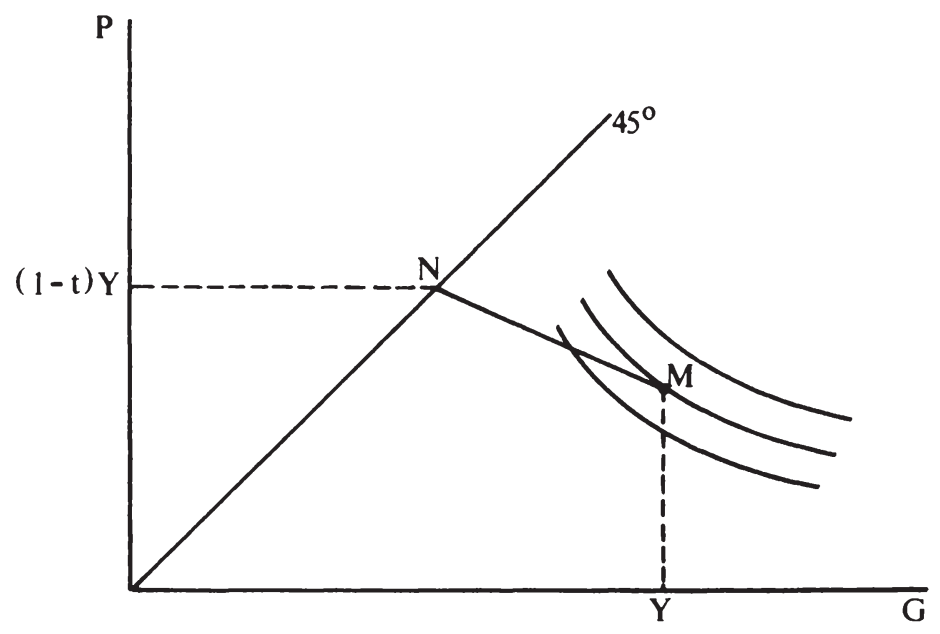

System von strikt konvexen Indifferenz-Kurven $\overline{E(U)}=q U(G)+p U(P)$ beschreiben, wobei $\overline{E(U)}$ jeweils ein gegebener Erwartungsnutzen ist. Die Steigung der Indifferenzkurve in Punkt $N$ ist gleich

$$
\frac{d P}{d G}=-\frac{q U^{\prime}(G)}{p U^{\prime}(P)}=-\frac{q}{p}
$$

da auf der $45^{\circ}$-Linie $P=G$ gilt. $q-p \alpha>0$ ist gleichbedeutend mit $-\alpha>$ $-q / p$. Die Steigung der Indifferenzkurve in $N$ ist somit kleiner als die Steigung der Budgetgeraden. Es gibt daher Kombinationen von $P$ und $G$ mit $P<G$ (bzw. $H>0$ ), die zu einem höheren Erwartungsnutzen als in Punkt $N$ führen.

Die optimale Wahl $P^{*}$ und $G^{*}$ ist für eine innere Lösung durch die Tangentialbedingung

$$
\frac{q U^{\prime}\left(G^{*}\right)}{p U^{\prime}\left(P^{*}\right)}=\alpha
$$

charakterisiert. Diese Bedingung ergibt sich unabhängig davon, ob man $H, D$ oder $P$ und $G$ als Entscheidungsvariable verwendet. Sie ist in Abbildung 1.1 in $O$ erfüllt. Der horizontale Abstand $O N$ mißt die hinterzogene Steuer $t H^{*}$. Der vertikale Abstand $N O$ ist gleich der Strafe $\alpha t H^{*}$.

Bei der Ecklösung $H^{*}=Y$ gilt für die Steigung der Indifferenzkurve durch $M$

$$
\frac{q U^{\prime}(G)}{p U^{\prime}(P)} \geqq \alpha
$$


Abbildung 1.2 zeigt den Fall, daß diese Ungleichung strikt gilt. Die Ecklösung ist bei einem vorgegebenen Einkommen nicht interessant, da sich das hinterzogene Einkommen bei kleinen Variationen der Parameter nicht ändert. Die folgende Analyse geht daher von einer inneren Lösung aus.

Die Bedingung erster Ordnung (1.5) bestimmt $H^{*}$ in Abhänigkeit von den Parametern des Modells:

$$
H^{*}=H^{*}(p, \alpha, t, Y)
$$

Setzt man diese Funktion in (1.5) ein, erhält man die Identität

$$
E_{H}\left(p, \alpha, t, Y, H^{*}(p, \alpha, t, Y)\right) \equiv 0
$$

Durch Differentiation dieser Identität erhält man die Ableitungen von $H^{*}$ nach den drei finanzpolitisch beeinflußbaren Parametern und dem vorgegebenen Einkommen.

Eine Erhöhung der Entdeckungswahrscheinlichkeit bewirkt

$$
\frac{\partial H^{*}}{\partial p}=-\frac{\partial E_{H}}{\partial p} / \frac{\partial E_{H}}{\partial H}=\Delta^{-1}\left(U^{\prime}(G)+U^{\prime}(P) \alpha\right)<0
$$

wobei $\Delta$ für $q U^{\prime \prime}(G) t+p U^{\prime \prime}(P) t \alpha^{2}$ steht. Steigt die Wahrscheinlichkeit, steuerlich geprüft zu werden, wird weniger Einkommen hinterzogen. Eine entsprechende Aussage gilt für die Verschärfung der Strafe:

$$
\frac{\partial H^{*}}{\partial \alpha}=\Delta^{-1}\left(p U^{\prime}(P)-p U^{\prime \prime}(P) t \alpha H^{*}\right)<0
$$

Da $t$ und $Y$ konstant sind, lassen sich die Reaktion des hinterzogenen Einkommens und der hinterzogenen Steuer unmittelbar ableiten. Wegen $D^{*}=Y-H^{*}$ gilt $\partial D^{*} / \partial p=-\partial H^{*} / \partial p$. Weiter ist $\partial\left(t H^{*}\right) / \partial t=t \partial H^{*} / \partial p$.

Die Ergebnisse lassen sich graphisch darstellen. Ausgehend von $O_{1}$ in Abbildung 1.3 dreht ein höherer Strafsatz die Budgetgerade um $N$ nach unten. Der Stpfl. wählt nun $\mathrm{O}_{2}$. Die hinterzogene Steuer sinkt: Der horizontale Abstand $\mathrm{O}_{2} \mathrm{~N}$ ist kleiner als der horizontale Abstand $O_{1} \mathrm{~N}$. Eine größere Entdeckungswahrscheinlichkeit ändert die Steigung der Indifferenzkurven. Durch $O_{1}$ in Abbildung 1.4 verläuft mit höherem $p$ eine Indifferenzkurve $\widetilde{E(U)}$, die einen geringeren Erwartungsnutzen als $\overline{E(U)}$ aufweist. Die neue Wahl $\mathrm{O}_{2}$ beinhaltet geringere hinterzogene Steuern.

Wir können kurz festhalten: Kontrollen und Strafen sind in dem Modell wirksame Mittel, um die Steuerhinterziehung zu begrenzen. Ob insbesondere Strafen Gestzesverstöße erfolgreich eindämmen können, wird oft bezweifelt ${ }^{9}$. In dem speziellen Fall des Steuerbetrugs sind die Zweifel nicht berechtigt, wenn man dem

\footnotetext{
${ }^{9}$ Einen Überblick zu den Arbeiten von Ökonomen zu dieser Frage geben Cameron (1988) und Tullock (1974).
} 
Abbildung 1.3: Die Strafe steigt.

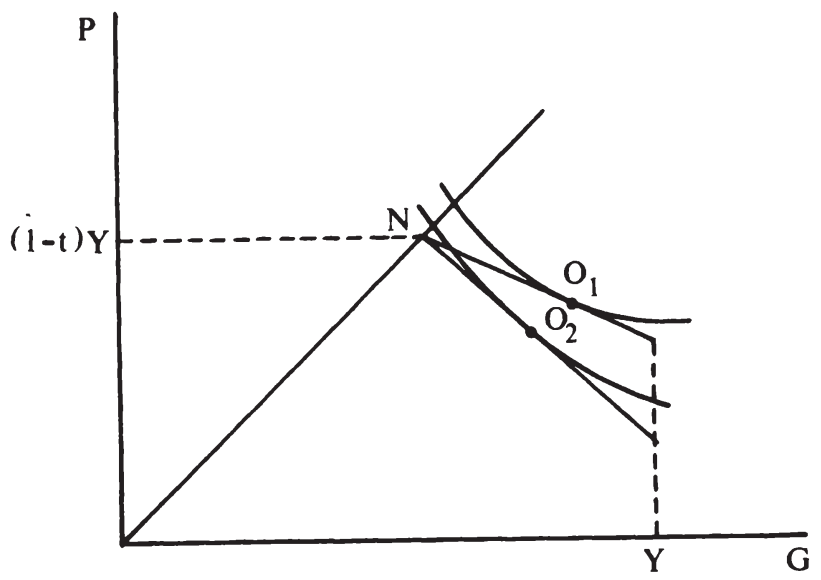

Abbildung 1.4: Die Entdeckungswahrscheinlichkeit steigt.

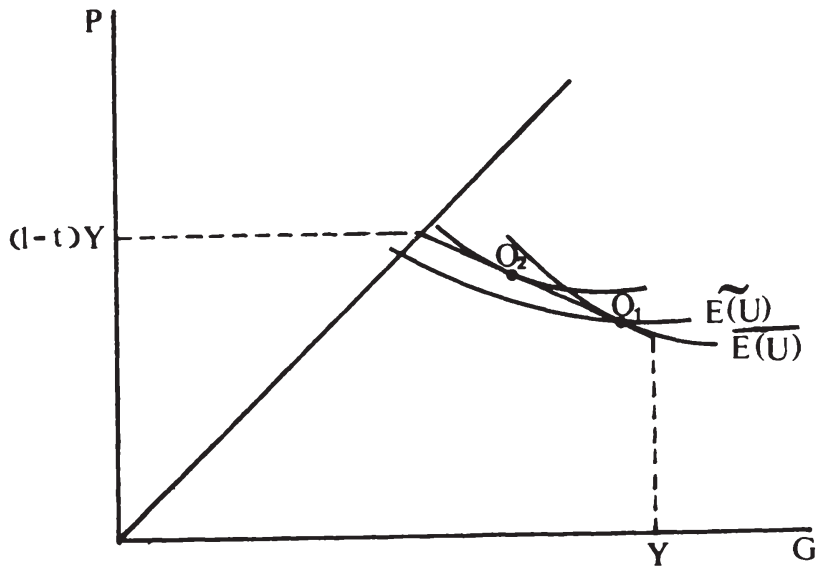


Grundmodell Glauben schenken will. "The reason is perfectly simple: Demand curves slope downward. If you increase the cost of something, less will be consumed. Thus, if you increase the cost of committing a crime (e.g. tax evasion (R.H.)), there will be fewer crimes" (Tullock, 1974, S. 104 f.). Die Erweiterungen des Grundmodells werden die Frage der Effektivität von Strafen und Kontrollen weiter verfolgen.

Es bleibt zu untersuchen, wie die Besteuerung und das Bruttoeinkommen auf die Steuerhinterziehung wirken. Die Wirkung eines höheren Steuersatzes scheint auf den ersten Blick nicht sehr interessant zu sein, da über den Zusammenhang zwischen der Höhe der Steuern und dem Steuerwiderstand recht dezidierte Vorstellungen bestehen, die z.B. Kaldor (1956, S. 158) und Gutmann (1977, zitiert nach Clotfelter, 1983, S. 363) wie folgt ausdrücken: "The extent to which people are willing to ... evade tax is much less, when $45 \%$ of the gains made from any particular transaction can be saved by evasion than when nine-tenths of the amount can be so saved". "Higher and higher taxes drive more and more of the economy underground, beyond the reach of the tax collector". Das Modell liefert jedoch

$$
\frac{\partial H^{*}}{\partial t}=\Delta^{-1}\left(q U^{\prime \prime}(G)(Y-H)-p U^{\prime \prime}(P) \alpha(Y+\alpha H)\right) \gtreqless 0
$$

Die Wirkung einer höheren Steuer auf das hinterzogene Einkommen ist im allgemeinen unbestimmt. Durch eine Spezifizierung der Erwartungsnutzenfunktion kann sie jedoch bestimmt werden.

Die Analyse wirtschaftlichen Verhaltens unter Unsicherheit hat neben der Risikoaversion von Anfang an zusätzliche Annahmen über die Präferenzen der Entscheidungsträger eingeführt, um deren Handlungen zu prognostizieren (siehe Arrow, 1965). Arrows Hypothese einer mit dem risikolosen Einkommen abnehmenden absoluten und zunehmenden relativen Risikoaversion wird auch in den Analysen der Steuerhinterziehung verwendet. Im folgenden wird über das Maß der absoluten Risikoaversion

$$
R_{a}(C)=-\frac{U^{\prime \prime}(C)}{U^{\prime}(C)}
$$

$R_{a}^{\prime}(C)<0$ angenommen. An wenigen Stellen wird auch das Maß der relativen Risikoaversion

$$
R_{r}(C)=C R_{a}(C)
$$

verwand und dabei $R_{r}^{\prime}(C)>0$ angenommen ${ }^{10}$. Die beiden Teile von Arrows Hypothese werden im folgenden mit a.a.R. und z.r.R. abgekürzt.

\footnotetext{
${ }^{10}$ Eine theoretische Begründung dieser zwei Maße liefern Pratt (1964) und Yaari (1969). Der Aufsatz von Yaari ist besonders empfehlenswert, da er - wie das Grundmodell der Steuerhinterziehung - von zwei "states of nature" ausgeht.
} 
Abbildung 1.5: Besteuerung und Steuerhinterziehung

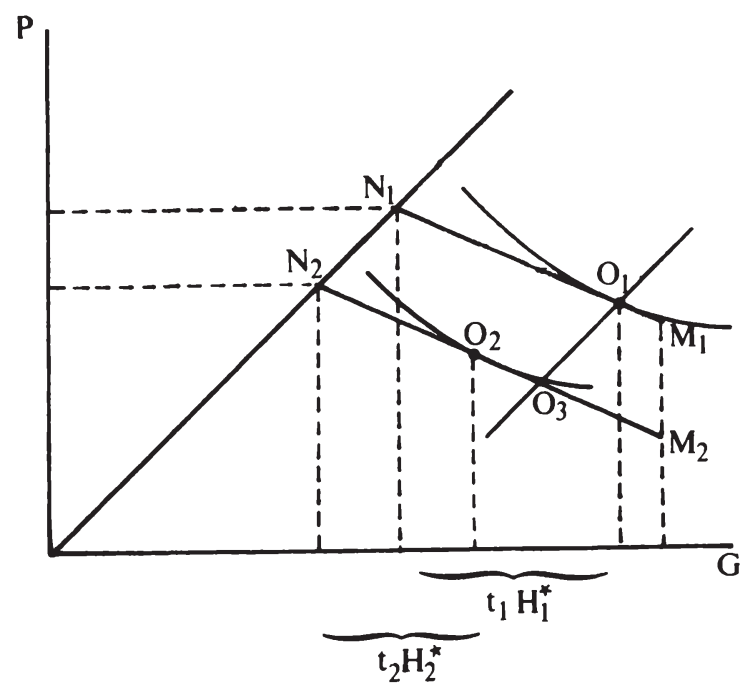

Mit einer a.a.R. senkt ein höherer Steuersatz das hinterzogene Einkommen. Substituiert man die Bedingung erster Ordnung (1.5) in (1.8), ergibt sich

$$
\frac{\partial H^{*}}{\partial t}=-\Delta^{-1} q U^{\prime}(G)\left[R_{a}(G)(Y-H)-R_{a}(P)(Y+\alpha H)\right]<0
$$

da $R_{a}(G)<R_{a}(P)$ ist wegen $G>P$ und $Y-H<Y+\alpha H$ ist. Weiter erhält $\operatorname{man}{ }^{11}$

$$
\frac{\partial\left(t H^{*}\right)}{\partial t}=-\frac{q U^{\prime}(G)\left(R_{a}(G)-R_{a}(P)\right) Y}{q U^{\prime \prime}(G)+U^{\prime \prime}(P) \alpha^{2}}<0
$$

Eine höhere Steuer verschiebt in Abbildung 1.5 die Budgetgerade $N_{1} M_{1}$ nach $\mathrm{N}_{2} \mathrm{M}_{2}$. Mit einer konstanten absoluten Risikoversion wäre der Stpfl. bei jedem sicheren Nettoeinkommen $(1-t) Y$ bereit, eine gleich hohe riskante Position gegenüber der Finanzbehörde zu übernehmen. Die riskante Position ist die Steuerersparnis auf der einen, die Strafe auf der anderen Seite. Mit $R_{a}(G)=R_{a}(P)$ würde sich der Haushalt ausgehend von $O_{1}$ entlang der Geraden $O_{1} O_{3}$ bewegen, wenn der Steuersatz steigt. $O_{1} O_{3}$ verläuft parallel zu der $45^{\circ}$-Achse. Jeder Punkt auf dieser Geraden ist mit einer gleich hohen riskanten Position verbunden. So ist

\footnotetext{
${ }^{11}$ Die Reaktion der hinterzogenen Steuer kann man am einfachsten berechnen, indem man in dem Optimierungsproblem des Stpfl. $S=t H$ als Entscheidungsvariable substituiert. Die Nebenbedingung lautet dann $0 \leqq S \leqq t Y$.
} 
z.B. der horizontale Abstand $O_{1} N_{1}$ gleich dem horizontalen Abstand $O_{3} N_{2}$. Eine a.a.R. bedeutet nun, daß der Stpfl. mit geringerem sicheren Einkommen $(1-t) Y$ die absolute Höhe seiner riskanten Position verringert. Mit $t_{2}>t_{1}$ wählt der Stpfl. einen Punkt auf $\mathrm{N}_{2} \mathrm{M}_{2}$, der links von $\mathrm{O}_{3}$ liegt, also z.B. $\mathrm{O}_{2}$. Die hinterzogene Steuer und die Strafe sind in $O_{2}$ geringer als in $O_{1}: t_{2} H_{2}^{*}<t_{1} H_{1}^{*}$. Wegen $t_{2}>t_{1}$ muß folglich das hinterzogene Einkommen sinken $\left(H_{2}^{*}<H_{1}^{*}\right)$, wenn $t$ steigt.

Eine exogene Erhöhung des Bruttoeinkommens führt zu einem steigenden hinterzogenen Einkommen, wenn man eine a.a.R. annimmt. Das sichere Nettoeinkommen steigt mit $Y$. Mit steigendem Nettoeinkommen wählt der Stpfl. aber eine größere riskante Position. Dies ist ein Ergebnis, das man intuitiv erwartet hat. Es beruht auf einem Verlauf der absoluten Risikoaversion, der bezüglich des $\mathrm{Zu}$ sammenhangs von Steuersatz und -hinterziehung zu einem unerwarteten Resultat führte.

Eine Aussage über das deklarierte Einkommen kann man nicht unmittelbar ableiten, wenn $Y$ variiert. Wenn das Einkommen z.B. um 10.000 DM zunimmt, und das hinterzogene Einkommen daraufhin um 7.000 DM steigt, nimmt das versteuerte Einkommen um 3.000 DM zu. Steigt $H^{*}$ dagegen um 12.000 DM, nimmt $D^{*}$ um 2.000 DM ab. Für die Änderung des versteuerten Einkommens kann man mit der Information $\partial H^{*} / \partial Y>0$ lediglich eine Obergrenze angeben:

$$
\frac{\partial D^{*}}{\partial Y}=1-\frac{\partial H^{*}}{\partial Y}<1
$$

Das heißt aber nichts weiter, als daß zumindest ein Teil des zusätzlichen Einkommens nicht versteuert wird - und das wissen wir bereits. Mit der Annahme einer z.r.R. kommt man zu einer Aussage über das versteuerte Einkommen (vgl. Allingham/Sandmo, 1972, S. 328). Man kann zunächst

$$
\frac{\partial\left(H^{*} / Y\right)}{\partial Y}=\frac{\left(\partial H^{*} / \partial Y\right) Y-H^{*}}{Y^{2}}=\frac{q U^{\prime}(G)\left(R_{r}(G)-R_{r}(P)\right)}{\Delta Y^{2}}
$$

berechnen. Der Anteil des hinterzogenen an dem tatsächlichen Einkommen sinkt mit $Y$. Aus $D^{*}=\left(1-H^{*} / Y\right) Y$ folgt dann

$$
\frac{\partial D^{*}}{\partial Y}=1-\frac{H^{*}}{Y}-Y \frac{\partial\left(H^{*} / Y\right)}{\partial Y}>0
$$

Das deklarierte Einkommen steigt mit dem Bruttoeinkommen.

Wir haben gesehen, daß das Bruttoeinkommen und der Steuersatz eine entgegengesetzte Wirkung auf die Steuerhinterziehung haben. Yitzhaki (1987) präzisiert diesen Zusammengang: Die hinterzogene Steuer ist eine Funktion

$$
S^{*}=f(p, \alpha, t, Y)=S^{*}(p, \alpha,(1-t) Y)
$$

Sie hängt bei gegebenen $p$ und $\alpha$ von dem sicheren Einkommen ab. 
Tabelle 1.1: Komparativ-statische Ergebnisse des Grundmodells

(Eine hochgestellte eins bedeutet, daB das Vorzeichen von einer a.a.R. abhängt. Bei einer zwei ist eine Z.r.R. angenommen. Wegen $H / Y=1-D / Y$ gilt $\left.d\left(D^{*} / Y\right)=-d\left(H^{*} / Y\right)\right)$.

\begin{tabular}{c|ccccc} 
& $H^{*}$ & $t H^{*}$ & $D^{*}$ & $H^{*} / Y$ & $A$ \\
\hline$p$ & $<0$ & $<0$ & $>0$ & $<0$ & $>0$ \\
$\alpha$ & $<0$ & $<0$ & $>0$ & $<0$ & $>0$ \\
$t$ & $<0^{1}$ & $<0^{1}$ & $>0^{1}$ & $<0^{1}$ & $>0^{1}$ \\
$Y$ & $>0^{1}$ & $>0^{1}$ & $>0^{2},<1^{1}$ & $<0^{2}$ & $>0^{1,2}$
\end{tabular}

Die Ergebnisse des Grundmodells faßt Tabelle 1.1 zusammen. Neben den Größen $H^{*}, t H^{*}$ und $D^{*}$ ist noch der Erwartungswert des Aufkommens aus der Einkommensteuer und den Strafen angegeben, der für den Fiskus von Interesse ist. Das erwartete Aufkommen von einem Stpfl.

$$
A=q t\left(Y-H^{*}\right)+p t\left(Y+\alpha H^{*}\right)=t Y-(q-p \alpha) t H^{*}
$$

ist gleich der Steuer auf das tatsächliche Einkommen abzüglich des erwarteten Ertrags der Hinterziehung für den Stpfl. Das Aufkommen umfaßt hier auch die Geldstrafe, da sie wie die Steuern zur Finanzierung staatlicher Ausgaben im weitesten Sinne verwandt wird. Es sei darauf hingewiesen, da $B A$ die Kosten der Steuererhebung nicht erfaßt. Insbesondere eine Erhöhung der (objektiven) Entdeckungswahrscheinlichkeit dürfte mit zusätzlichen Aufwendungen für die Steuerfahndung verbunden sein. Die Wirkung der Parameter auf $A$ bedarf keines Kommentars.

\subsection{Steuerhinterziehung und Steuervermeidung}

Das Grundmodell ist ohne weitere Diskussion eingeführt worden. Es bleibt daher die Aufgabe, die Tragweite des Modells zu bewerten. Dieser Aufgabe wenden sich dieser und der nächste Abschnitt zu. In Abschnitt 1.5 werden die Annahmen des Grundmodells diskutiert. Dabei wird deutlich werden, welche Modifikationen des Modells im weiteren Verlauf dieser Arbeit zu erwarten sind. Der Beantwortung der Frage, auf welche Aktivitäten eines Stpfl. das Modell anwendbar ist, dient die Abgrenzung der Steuerhinterziehung von der Steuervermeidung in diesem Abschnitt.

Nicht alle Aktivitäten, die das Ziel haben, Steuern zu sparen, können als Steuerhinterziehung qualifiziert werden. Der Anwendungsbereich der in dieser Arbeit 
verwendeten Modelle ist daher begrenzt. Die Abgrenzung ist nicht ganz einfach. Sie ist auf der anderen Seite lohnend, da sie den Standort einer Theorie der Steuerhinterziehung innerhalb der steuertheoretischen Literatur deutlich macht.

Sowohl der Steuerhinterzieher als auch derjenige, der Steuern vermeiden will, versuchen, Geld zu sparen. Die Gemeinsamkeiten von Steuerhinterziehung und Steuervermeidung sind damit aber im allgemeinen zu Ende. Sie unterscheiden sich durch ihre gesetzliche Würdigung, den Informationsstand der Finanzbehörden, durch eine wichtige Eigenschaft der Budgetbeschränkung des Stpfl. und ihre Inzidenz. Die Unterscheidungsmerkmale sind nicht unabhängig voneinander. Ich gehe jedoch im folgenden auf jedes der vier Merkmale ein, da erst durch alle zusammen deutlich wird, wie sich die Steuerhinterziehung von der Steuervermeidung unterscheidet.

Nach $\S 370$ Abs. 1 AO ${ }^{12}$ begeht eine Steuerhinterziehung, "wer

1. den Finanzbehörden oder anderen Behörden über steuerlich erhebliche Tatsachen unrichtige oder unvollständige Angaben macht,

2. die Finanzbehörden pflichtwidrig über steuerlich erhebliche Tatsachen in Unkenntnis läßt

... und dadurch Steuern verkürzt oder für sich oder einen anderen nicht gerechtfertigte Steuervorteile erlangt". Die Steuerhinterziehung ist illegal und wird bestraft ( 3370 Abs. 1, 2 und $3 \mathrm{AO}$ ).

Eine mit $\S 370$ AO vergleichbare gesetzliche Definition der Steuervermeidung gibt es nicht. Das hat zwei Gründe: Zum einen sind viele Formen der Steuervermeidung entweder gesellschaftlich erwünscht oder müssen vom Fiskus aus verschiedenen Gründen akzeptiert werden. Die Heirat etwa führt infolge des Splittingverfahrens zu einer Steuerersparnis. Diese Steuervermeidung ist erwünscht. Die Einkommensteuer kann eine Substitution von Freizeit für Einkommen verursachen. Die durch diese Substitution vermiedene Steuer muß der Gesetzgeber in Kauf nehmen, wenn er eine Einkommensteuer erheben will. Soweit der Gesetzgeber die Steuervermeidung nicht unterbinden will oder kann, kommt er ohne eine gesetzliche Definition des Tatbestandes aus.

Für die vom Gesetzgeber nicht gewollte Steuervermeidung (die Steuervermeidung im engeren Sinne) gibt es vielleicht deshalb keine gesetzliche Definition, weil er bei Abfassung der Steuergesetze noch nicht weiß, welche "Lücken" sich in ihnen verbergen und welcher Art die ökonomischen Transaktionen und rechtlichen Konstruktionen sein werden, mit deren Hilfe die Stpfl. die Steuer zu umgehen versuchen. Eine Definition der Steuervermeidung im engeren Sinne gibt Sandford

\footnotetext{
${ }^{12}$ Für die gesetzliche Abgrenzung ziehe ich deutsches Recht heran. Sie ist jedoch in fast gleicher Form in Rechtssystemen anderer Länder zu finden (siehe Tipke/Kruse, 1988, S. 90 - 93).
} 
(1980, S. 153): Sie bezeichnet "legal actions undertaken to save tax, which would not have been undertaken but for the tax, which enabled the actor to achieve substantially what he would habe archieved in the absence of the tax, and which actions are contrary to the intention of the government" (siehe auch Schneeloch, 1986, S. 260). Nach dieser Definition wäre Steuervermeidung legal. Sie bewegt sich im Gegensatz zu der Steuerhinterziehung innerhalb des Rechts. Dem entspricht die Formel der Rechtsprechung, das Steuerrecht respektiere grundsätzlich die von den Stpfl. gewählte rechtliche Gestaltung eines wirtschaftlichen Vorgangs (vgl. Tipke/Kruse, 1988, S. 72).

§ $42 \mathrm{AO}$ läßt an der Legalität der Steuervermeidung jedoch Zweifel aufkommen. Trotz des Mangels einer gesetzlichen Definition wird der Steuervermeidung dort nämlich eine Grenze gesetzt: "Durch den Mißbrauch von Gestaltungsmöglichkeiten des Rechts kann das Steuerrecht nicht umgangen werden. Liegt ein Mißbrauch vor, so entsteht der Steueranspruch so, wie er bei einer den wirtschaftlichen Vorgängen angemessenen rechtlichen Gestaltung entsteht". Der Gesetzgeber behält sich mit $\S 42 \mathrm{AO}$ vor, zumindest einen Teil der nicht gewollten Steuervermeidung als mißbräuchliche Steuervermeidung bzw. als Steuerumgehung (ebenda, S. 72) einzustufen. Die Folge ist, daß bestimmte aufgrund außersteuerlichen Rechts (insbesondere zivilrechtlich) zulässige Gestaltungen steuerlich nicht anerkannt werden. Dies heißt aber nicht, daß sie illegal sind und bestraft werden. Sie haben nur nicht die beabsichtigten steuersparenden Konsequenzen: Umgangene Steuern sind nachzuzahlen.

Steuerhinterziehung und Steuervermeidung lassen sich damit erstens wie folgt unterscheiden. Die Steuerhinterziehung ist illegal und wird bestraft. Die Steuervermeidung ist entweder legal oder sie ist nach außersteuerlichem Recht zwar legal, wird aber steuerlich nicht anerkannt.

Das zweite Unterscheidungsmerkmal betrifft den Informationsstand des Fiskus. Bei der Steuervermeidung kennt er alle "steuerlich erheblichen Tatsachen". Die Steuerhinterziehung besteht gerade darin, dem Fiskus unvollständige Angaben zu machen. Vollständig informiert kann dieser erst dann sein, wenn eine steuerliche Prüfung erfolgreich abgeschlossen ist.

Cowell (1987, S. 175) grenzt die Steuerhinterziehung von der Steuervermeidung durch eine Eigenschaft der Budgetbeschränkung des Stpfl. ab. Die Steuerhinterziehung resultiert in einer Unsicherheit über die letztlich zu zahlende Steuer und Strafe. Das Nettoeinkommen ist abhängig davon, ob der Steuerpflichtige (zufällig) entdeckt wird oder nicht. Die Steuervermeidung hat dagegen nach Cowell jeweils ein ganz bestimmtes, sicheres Einkommen nach Steuer zur Folge. Die Unterscheidung ist instruktiv, hat jedoch Grenzen. Die Wahrscheinlichkeit einer Kontrolle kann für einen Hinterzieher subjektiv (oder auch objektiv) null sein. Die Budgetbeschränkung wäre dann nicht stochastisch. Dennoch bleibt die Täuschung 
der Finanzbehörde nach dem Buchstaben des Gesetzes eine Steuerhinterziehung. Schwerer wiegt, daß auch die Steuervermeidung eine Entscheidung bei Unsicherheit sein kann. Gewöhnlicherweise eingeschlagene Wege der Steuervermeidung können verstellt sein, da sie plötzlich als Mißbrauch eingestuft werden. Neuen Gestaltungen kann von Anfang an die Anerkennung versagt bleiben, so daß Steuern nachzuzahlen sind und sich der (vielleicht beträchtliche) Aufwand nicht gelohnt hat. Das weiß man jedoch vielleicht erst am Ende eines Finanzgerichtsprozesses. Wenn sich steuerliche Gestaltungen an der Grenze zum Mißbrauch bewegen, ist ihr Erfolg unsicher (vgl. Stiglitz, 1986, S. 511).

Kommen wir zu dem letzten Unterscheidungsmerkmal. Die Steuerhinterziehung hängt von dem Erhebungsverfahren ab. Die Steuervermeidung hängt davon ab, ob es dem Gesetzgeber gelingt, die von ihm beabsichtigte Besteuerung gesetzlich zu verankern. "The incidence of evasion is ... a function of the mechanisms by which tax is assessed and collected, and the extent to which they can be controlled and monitored; the incidence of avoidance is a function of the tax base and depends on the extent to which legislation is successful in expressing the underlying economic concepts. Avoidance depends on the base, evasion on the assessment procedures" (Kay, 1980, S. 136).

Obwohl nicht jedes Unterscheidungsmerkmal in jedem Fall brauchbar ist, tragen sie alle dazu bei, die Steuerhinterziehung von der legalen Steuervermeidung und der Steuervermeidung, die sich in Richtung auf $\S 46$ AO bewegt (Steuerumgehung), abzugrenzen. Diese Dreiteilung der Aktivitäten, die auf eine Steuerersparnis abzielen, verwende ich jetzt, um den Platz einer Theorie der Steuerhinterziehung innerhalb der steuertheoretischen Literatur zu bestimmen.

Die traditionelle Steuerwirkungslehre und die Optimalsteuertheorie behandeln die legale Steuervermeidung durch "angemessene Gestaltung" des Rechts. Die Entscheidungen zwischen dem Arbeitsangebot und der Freizeit, zwischen Konsum und Sparen, die Entscheidungen über Finanzierung und Investitionen etc. werden von den Möglichkeiten beeinflußt, legal Steuern zu sparen. Seit Allingham/Sandmo (1972) beschäftigt sich die Steuertheorie auch mit der Hinterziehung. Sehr schnell ist sie dann auch dazu übergegangen, den Zusammenhang zwischen der angemessenen Steuervermeidung und der Steuerhinterziehung zu untersuchen, indem sie in Modellen mit endogenem Arbeitseinkommen die Steuerhinterziehung integrierte (siehe Abschnitt 2.2). Nur wenig Aufmerksamkeit hat die Finanzwissenschaft jedoch der Steuervermeidung im engeren Sinne gezollt, obwohl deren praktische Bedeutung nicht $\mathrm{zu}$ übersehen ist ${ }^{13}$. Mögliche Zusammenhänge zwischen der

\footnotetext{
${ }^{13}$ Kay (1980, S. 135) kommt zu der Einschätzung, “... it is avoidance activity (im Sinne eines tendenziellen Mißbrauchs von Gestaltungsmöglichkeiten (R.H.)), which accounts for a high proportion of the economic effects of taxation, rather than the work/leisure or consumption/savings tradeoffs conventionally analysed in public finance”. Auch wenn man Kays Einschätzung nicht
} 
Steuervermeidung im engeren Sinne (Steuerumgehung) und der Steuerhinterziehung sind bislang fast völlig unbeachtet geblieben. Offensichtlich ist natürlich, da $B$ niemand Steuern hinterziehen wird, die er mit geringem Aufwand vermeiden kann, ohne an die Grenze des $\S 42 \mathrm{AO}$ zu stoßen ${ }^{14}$.

\subsection{Die Prämissen des Grundmodells - eine Diskussion}

Dieser Abschnitt läßt die Annahmen des Grundmodells Revue passieren. Den anschließenden Kommentaren zu den einzelnen Annahmen kann entnommen werden, welche Annahmen in den folgenden Kapiteln beibehalten und welche modifiziert werden.

Die Annahmen des Grundmodells lauteten:

1. Es wird das Partialmodell eines Stpfl. behandelt.

2. Die Präferenzen des Stpfl. sind ausschließlich über Konsum definiert. Sie genügen den Axiomen der Erwartungsnutzentheorie und Arrows Hypothese einer abnehmenden absoluten Risikoaversion.

3. Der Stpfl. verfügt über ein fest vorgegebenes Einkommen, das ihm legal zugeflossen ist.

4. Er unterliegt einer Einkommensteuer mit proportionalem Tarif.

5. Der Stpfl. deklariert zuerst sein Einkommen und zahlt die veranlagte Steuer. Anschließend kann eine Prüfung erfolgen, die bei entdeckter Steuerhinterziehung zu einer Nachzahlung und einer Strafe führt.

6. Das hinterzogene Einkommen der betrachteten Periode ist die einzige Entscheidungsvariable des Stpfl.

7. Die Hinterziehung verursacht bis auf das mit ihr verbundene Risiko keine Kosten.

teilt, bleibt die theoretische Analyse der Steuerumgehung eine wichtige Aufgabe. Auf vier der wenigen vorliegenden Arbeiten sei daher hingewiesen: Feldman/Kay (1981), Kay (1980), Stiglitz (1983) und (1986).

${ }^{14} \mathrm{Ob}$ weitergehende Zusammenhänge existieren, die eine theoretische Analyse lohnend machen, ist offen. Ein Modell, das Steuerhinterziehung und -vermeidung zusammen erfassen soll, präsentieren Cross/Shaw (1982). Sie postulieren einfach Beziehungen zwischen Steuerhinterziehung und -vermeidung durch eine gemeinsame Kostenfunktion beider Aktivitäten. 
8. Die Steuerprüfung erfolgt mit einer Wahrscheinlichkeit $0<p<1$ und deckt das tatsächliche Einkommen des Stpfl. auf.

9. Die Strafe ist ein konstantes Vielfaches der hinterzogenen Steuer.

Die Ausführungen zu den einzelnen Annahmen sind entsprechend numeriert.

ad 1) Das Partialmodell ist in der mikroökonomischen Theorie stets der erste Schritt. In der Theorie der Steuerhinterziehung ist es allerdings bislang überwiegend bei Partialmodellen geblieben. Die Kapitel 3 bis 6 dieser Arbeit machen da keinen Unterschied. Über Ansätze, die über das Partialmodell hinausgehen, wird in Kapitel 2 berichtet.

ad 2) Der erste Teil von Annahme 2 erstaunt: Warum sind neben dem privaten Konsum nicht auch staatliche Ausgaben für öffentliche Güter Argument der Nutzenfunktion, wenn der Staat Steuern beansprucht und es ihm gelingt, diesen Anspruch zum Teil durchzusetzen. Die Antwort ist, daß öffentliche Güter ein Argument der Nutzenfunktion sein werden. Wenn das Ausmaß der Bereitstellung öffentlicher Güter jedoch für den einzelnen eine konstante Größe ist, müssen sie in der Analyse eines repräsentativen Stpfl. nicht berücksichtigt werden. Dieser wird die staatlichen Ausgaben als unabhängig von der Höhe seiner Steuerzahlung betrachten und, sofern er individuell-rational handelt, versuchen, Steuern zu verkürzen: Der Steuerhinterzieher ist ein Trittbrettfahrer par excellence.

Die Erwartungsnutzentheorie ist in ihrer hergebrachten Form vielen Einwänden ausgesetzt. Als Hypothese über das tatsächliche Entscheidungsverhalten der Individuen ist sie in vielen Fällen nicht brauchbar, wie Schoemaker (1982, S. 552) in seinem Überblick feststellt. "However, there may be exceptions. For well-structered repetitive tasks, with important stakes, and well trained decision makers, expected utility maximization may well describe the actual decision process" (ebenda, S. 552). Zumindest die zwei zuerst genannten Bedingungen sind bei der jährlichen Steuerveranlagung erfüllt. Ein weiterer Grund, in dieser Arbeit der traditionellen Erwartungsnutzenhypothese zu folgen, ist, daß Präferenzfunktionen, die sich nicht allzu sehr von der Erwartungsnutzenfunktion unterscheiden, zu qualitativ gleichen komparativ-statischen Ergebnissen führen (siehe Machina, 1989).

Laut Annahme 2 beeinflussen nichtpekuniäre Faktoren den Stpfl. nicht (vgl. Allingham/Sandmo 1972, S. 326). Die Steuerhinterziehung ist nicht mit psychischen Kosten oder Erträgen verbunden. Vorstellbar ist natürlich, daß der soziale Status durch eine Verurteilung beeinträchtigt wird (Andersen, 1977, S. 377). Genausogut ist auch möglich, daß eine erfolgreiche Hinterziehung zu einer steigenden Reputation bei engen Freunden führt. Technisch gesprochen wäre der Nutzen in diesen Fällen zustandsabhängig. Die ökonomische Theorie tut sich schwer damit, Aussagen über psychologische Determinanten der Steuerhinterziehung zu machen. Die Frage ist, ob sie durch die Konzentration auf pekunäre Faktoren einen großen 
Fehler macht. Schließlich ist die Steuerhinterziehung ein anonymes Delikt: Ein menschliches Opfer wie z.B. bei einem Raubüberfall ist für den Täter nicht erkennbar. Eine Verurteilung bleibt Dritten zumeist verborgen. Daher ist es bei der Steuerhinterziehung - wie bei anderen Arten der Wirtschaftskriminalität - in vielen Fällen gerechtfertigt, ausschließlich Faktoren zu betrachten, die sich in Geld niederschlagen. An einer Stelle wird jedoch auch ein Modell dargestellt, in dem die Steuermoral der Gesellschaft Einfluß auf jeden einzelnen hat.

Aus Annahme 2 und den unterstellten steuerrechtlichen Kenntnissen des Haushalts folgt, daß eine Hinterziehung nie unbeabsichtigt (ohne Vorsatz) erfolgt. Für eine positive Analyse ist dies nicht problematisch. Interessant wird die Unterscheidung zwischen vorsätzlichen und versehentlichen Gesetzesverstößen, wenn der Staat Kontrollen und Strafen nach einem bestimmten Kriterium optimal festsetzen will, aber zwischen den beiden Typen nicht (gut) unterscheiden kann: Nur hohe Strafen haben einen abschreckenden Effekt auf die Täter aus Vorsatz; sie führen aber zu unerwünschten Nebenwirkungen (sie sind z.B. ungerecht), wenn ein Verstoß nur "leichtfertig" begangen wurde. Auf dieses Dilemma komme ich in Kapitel 6 zurück.

ad 3) Annahme 3 bedeutet, daß die ökonomischen Aktivitäten, durch die der Stpfl. sein Einkommen verdient, unabhängig von den Möglichkeiten sind, Steuern zu hinterziehen. Kurzfristig kann diese Annahme durchaus sinnvoll sein. Langfristig gibt es zwischen der Steuerbelastung bzw. den Möglichkeiten, der Besteuerung zu entgehen, und der Entstehung des Einkommens im allgemeinen einen Zusammenhang. Die Entscheidungen der Stpfl. über ihr Arbeitsangebot und ihre Ersparnis sind abhängig von der zu erwartenden Steuerlast. Ein Schwerpunkt der Literatur und dieser Arbeit liegt daher bei der Berücksichtigung des Zusammenhangs von Entstehung der Besteuerungsgrundlage und der Hinterziehung.

ad 4) Der Untersuchung eines proportionalen folgt stets die Analyse eines progressiven Tarifs. Siehe dazu Abschnitt 2.1. Für die Bezeichnung der Bemessungsgrundlage bzw. den Namen der untersuchten Steuer gibt es, wie bereits erwähnt wurde, verschiedene Möglichkeiten, solange die Bemessungsgrundlage exogen ist. Ist sie endogen, unterscheiden sich die Modelle der Steuerhinterziehung je nach untersuchter Steuer. Eine Systematik der Modelle mit endogener Bemessungsgrundlage enthält Abschnitt 2.2.

ad 5) Annahme 5 betrifft die zeitliche Struktur des Modells. Diese zeitliche Abfolge ist immer gegeben, solange die Steuererhebung auf die Mitarbeit einer (oder mehrerer) Wirtschaftseinheit(en) des privaten Sektors (des Steuerzahlers und/oder eines zum Abzug Verpflichteten) angewiesen ist. Sie ist daher in allen Modellen der Steuerhinterziehung anzutreffen.

In der Praxis kommt es gar nicht selten vor, daß veranlagte Steuern - sei die Bemessungsgrundlage richtig oder zu niedrig deklariert worden - nicht bezahlt 
werden. Den Finanzbehörden fehlt oft das Personal, um Steuerrückstände einzutreiben. Dieser Fall ist mit Annahme 5 ausgeschlossen.

ad 6) In Erweiterungen des Grundmodells wird das hinterzogene Einkommen einer einzigen Periode um weitere Entscheidungsvariable ergänzt. Es handelt sich erstens um ein endogenes Einkommen (bzw. eine endogene Steuerbemessungsgrundlage), zweitens um das hinterzogene Einkommen weiterer Perioden. Wenn der Stpfl. mehrere Perioden betrachtet, in denen er jeweils sein Einkommen deklarieren muß, wird es im allgemeinen nicht optimal sein, die Steuererklärungen für die verschiedenen Zeitabschnitte unabhängig voneinander abzugeben. Das ist insbesondere dann der Fall, wenn Kontrollen und Strafen von dem Verhalten des Stpfl. in der Vergangenheit abhängig sind. Diese Form der Steuersicherung behandelt Kapitel 6.

ad 7) Die Annahme einer bis auf das Risiko einer Nachzahlung und einer Strafe kostenlosen Hinterziehung ist z.B. für einen privaten Haushalt in der Bundesrepublik, der nur einen Teil seiner Zinserträge deklariert, gerechtfertigt, wenn eine Bestechung der Fahndungsbeamten nutzlos ist. Um hingegen einem Zoll zu entgehen, kann es notwendig sein, die Waren abseits der Handelswege durch unwegsames Gelände zu befördern, so daß zusätzliche Kosten entstehen. Die Literatur nimmt in der Regel eine kostenlose Hinterziehung an ${ }^{15}$. Ich folge dieser Praxis. Eine Berücksichtigung fixer Kosten der Hinterziehung oder variabler Kosten in Abhängigkeit von dem hinterzogenen Einkommen wäre allerdings nicht schwierig, würde jedoch nicht zu wesentlich anderen Ergebnissen führen.

ad 8) Die Entdeckungswahrscheinlichkeit muß nicht konstant sein. Sie kann von Informationen abhängen, die die Behörde schon vor einer Prüfung über den Stpfl. besitzt. Diese Möglichkeit wird kurz in Abschnitt 2.2 und ausführlich in Kapitel 6 untersucht.

ad 9) $\mathrm{Zu}$ der im Grundmodell verwendeten Strafe gibt es Alternativen. Ein Schwerpunkt der Literatur ist die Untersuchung der Konsequenzen verschiedener Strafen. In Kapitel 2 werden zwei weitere Straffunktionen vorgestellt. Kapitel 6 behandelt rückwirkende Strafen.

\subsection{Zusammenfassung}

Ob Steuern durch Veranlagung oder durch Quellenabzug erhoben werden - mindestens eine Wirtschaftseinheit hat die Möglichkeit, illegal Steuern zu sparen, da der Staat nicht über alle ökonomischen Transaktionen, die eine Steuerschuld begründen, informiert sein kann. Ob ein Steuerpflichtiger von der Möglichkeit der Hinterziehung Gebrauch macht, ist eine zweite Frage, wie das Grundmodell anhand

\footnotetext{
${ }^{15}$ Eine Ausnahme sind die Aufsätze zum Schmuggel (siehe z.B. Norton, 1988).
} 
eines Haushalts, der zur Einkommensteuer veranlagt wird, gezeigt hat. Die Steuerhinterziehung kann durch die Steuerfahndung aufgedeckt und bestraft werden, so daß sich ein Steuerpflichtiger, der nur an seinem Nettoeinkommen interessiert ist, fragen muß, ob sich die Steuerhinterziehung lohnt.

Wenn die Entdeckungswahrscheinlichkeit und der Strafsatz so klein sind, daß nicht das tatsächliche Einkommen deklariert wird, stellt sich die Frage, wie die Steuerhinterziehung auf eine Änderung finanzpolitischer Parameter reagiert. Die Analyse des Grundmodells hat neben erwarteten auch ein unerwartetes Resultat gebracht. Höhere Strafen und verschärfte Kontrollen haben eine abschreckende Wirkung auf einen rationalen Steuerhinterzieher. Ein höherer Steuersatz hat überraschenderweise ebenfalls diese Wirkung, wenn man eine abnehmende absolute $\mathrm{Ri}$ sikoaversion annimmt. Das letzte Ergebnis impliziert, daß das Steueraufkommen mit einem höheren Steuersatz steigt. Daher finanziert sich umgekehrt eine Steuersenkung nicht zum Teil von selbst: Die Wirkung eines kleineren Steuersatzes auf das Aufkommen bei gegebenem Umfang des hinterzogenen Einkommens wird nicht durch höhere deklarierte Einkommen kompensiert.

Die Steuerhinterziehung ist nur eine der möglichen Aktivitäten, um Steuern zu sparen. Sie ist zu unterscheiden von verschiedenen Formen der Steuervermeidung, die dieses Ziel auf legalem Wege erreichen können. Die Steuerhinterziehung ist eine naheliegende Gelegenheit, Steuern zu sparen, wenn der Staat die Steuerhinterziehung nicht unterbinden kann oder will und wenn alle Möglichkeiten, legal die Abgabenlast zu verringern, ausgeschöpft sind. Entsprechend ist die Theorie der Steuerhinterziehung seit der Arbeit von Allingham/Sandmo (1972) nicht mehr aus der Steuerwirkungslehre wegzudenken. Auf Entwicklungen dieser Theorie, die in den folgenden Kapiteln dieser Arbeit zu finden sind, hat eine Diskussion der Annahmen des Grundmodells bereits hingewiesen. 


\section{Kapitel 2}

\section{Erweiterungen des Grundmodells}

Dieses Kapitel enthält einen Überblick zu Modifikationen und Erweiterungen des Grundmodells, die in der Literatur zu finden sind. Den Erwartungen, die die Diskussion der Annahmen des Grundmodells in Abschnitt 1.5 geweckt hat, versuche ich wie folgt zu entsprechen. In Abschnitt 2.1 werden einzelne Bestandteile des Steuersystems variiert. Konkret handelt es sich dabei um alternative Formen der Geldstrafe, eine variable Entdeckungswahrscheinlichkeit und einen progressiven Steuertarif. Die Annahmen eines exogenen Einkommens und einer einzigen Entscheidungsvariablen werden beibehalten. Abschnitt 2.2 enthält eine Systematik der Ansätze mit einer endogenen Steuerbemessungsgrundlage. Abschnitt 2.3 weist auf die Überlegungen hin, die den partialanalytischen Rahmen überschreiten und Interdependenzen zwischen verschiedenen Steuerpflichtigen berücksichten. Eine wichtige jüngere Entwicklung wird erst an späterer Stelle aufgegriffen: Modelle mit wiederholter Veranlagung.

\subsection{Variationen des Steuersystems}

\subsubsection{Alternative Geldstrafen}

Die in dem Grundmodell verwendete Geldstrafe $\Phi_{1}=\alpha t H$ ist proportional zu der Bemessungsgrundlage $t H$. Die zwei folgenden Strafen setzen an diesen zwei Eigenschaften an. Die von Allingham/Sandmo (1972) verwendete Strafe

$$
\Phi_{2}=\beta H, \quad \beta>0
$$

hat statt der hinterzogenen Steuer das hinterzogene Einkommen als Bemessungsgrundlage, das sie mit einer Konstanten $\beta$ multipliziert. Die von Frost (1986) eingeführte "deutsche Strafe"

$$
\Phi_{3}=[\gamma(1-t) Y] t H, \quad \gamma>0
$$


behält die hinterzogene Steuer als Basis bei. Sie macht jedoch den Multiplikator, den die eckige Klammer enthält, abhängig von dem Nettoeinkommen des Stpfl. Die deutsche Strafe ist damit progressiv in dem Sinne, daß Stpfl. mit einem höheren Einkommen für jeweils gleich hohe hinterzogene Steuern eine höhere Geldstrafe zu zahlen haben.

Bei $\Phi_{2}$ und $\Phi_{3}$ handelt es sich wie bei $\Phi_{1}$ immer noch um spezielle Funktionen. Allgemeinere Formen sind denkbar. Die folgende Analyse der zwei zusätzlichen Geldstrafen reicht jedoch aus, um deutlich zu machen, daß die Steuerhinterziehung kritisch von der Form der Strafe abhängt ${ }^{1}$.

Freiheitsstrafen und weitere mögliche Konsequenzen der Steuerhinterziehung werden in dieser Arbeit nicht berücksichtigt. "Für die steuerstrafrechtliche Praxis dürfte davon auszugehen sein, daß ... in den Regelfällen der Steuerhinterziehung auf Geldstrafe allein zu erkennen ist" (Kühn/Kutter/Hofmann, 1987, Anm. 12 c) zu $§ 370 \mathrm{AO}$, S. 800).

\section{Die Strafe nach Allingham/Sandmo}

Mit $\Phi_{2}$ lautet die Budgetgleichung für den Fall, daß der Stpfl. geprüft wird ${ }^{2}$

$$
P=(1-t) Y-\beta H
$$

In Abweichung von Allingham/Sandmo (1972, S. 325) ist hier eine Nachversteuerung des hinterzogenen Einkommens angenommen. Der Konsum für den Fall, daß der Stpfl. Glück hat, ist unabhängig von der Form der Strafe gleich

$$
G=(1-t) Y+t H
$$

Durch geeignete Wahl von $H$ maximiert der Stpfl. den Erwartungsnutzen $E U(C)=$ $q U(G)+p U(P)$ unter der Nebenbedingung $0 \leqq H \leqq Y$. Die Bedingung erster Ordnung für eine innere Lösung ist

$$
q U^{\prime}(G) t-p U^{\prime}(P) \beta=0
$$

Aus (2.3) folgt, daß $H^{*}$ positiv ist, wenn $q t-p \beta>0$ gilt. Die Bedingung für ein positives hinterzogenes Einkommen ist mit $\Phi_{2}$ von der Höhe des Steuersatzes abhängig. Je größer der Steuersatz ist, desto eher kommt es zu einem Steuerbetrug. Das war mit der Strafe $\Phi_{1}$ anders.

\footnotetext{
${ }^{1}$ Angesichts dieser Aussage der Theorie ist es etwas verwirrend, wenn man in einem Buch über "Die Steuerfahndung", das zur rechtlichen Beratung der Stpfl. geschrieben ist, keine Ausführungen zu den Strafen findet, die bei Entdeckungen der Fahnder zu erwarten sind (Streck, 1986).

${ }^{2}$ Ich verzichte darauf, $P, G, H, D$ und $\Delta$ entsprechend der verwendeten Strafe, dem verwendeten Steuertarif etc. zu indizieren. Die Bedeutung der Variablen geht immer aus dem Zusammenhang hervor.
} 
Aus (2.3) kann man die Vorzeichen der Funktion $H^{*}=H^{*}(p, \beta, t, Y)$ ableiten. Die Wirkung von $p, \beta$ und $Y$ auf das hinterzogene Einkommen ändert sich durch den Ersatz von $\Phi_{1}$ durch $\Phi_{2}$ nicht. Diese Aussage erläutert die Budgetgerade

$$
P=(1-t) Y-\frac{\beta}{t}(G-(1-t) Y)
$$

die man aus (2.1) und (2.2) erhält. Ihre Steigung $\partial P / \partial G=-\beta / t$ ist der Preis der Hinterziehung: Für jede DM Steuern, um die der Stpfl. den Fiskus betrügt, muß er eine Strafe $\beta / t$ zahlen, falls er erwischt wird. Aufgrund unserer Kenntnis des Grundmodells läßt sich folgern: (i) Eine Erhöhung von $p$ verschiebt das Indifferenzkurvensystem so, da $B$ im neuen Gleichgewicht ein geringeres Einkommen hinterzogen wird. (ii) Eine Erhöhung des Strafsatzes $\beta$ erhöht den Preis der Hinterziehung, so daß das hinterzogene Einkommen sinkt. (iii) Ein steigendes Einkommen verschiebt die Budgetgerade parallel nach oben. Unter der Annahme einer a.a.R. steigt $H^{*}$. Auch in bezug auf die hinterzogenen Steuern, das deklarierte Einkommen und das erwartete Steueraufkommen gelangt man mit der Strafe von Allingham/Sandmo bis auf eine Ausnahme zu den gleichen qualitativen Aussagen wie bei der Verwendung von $\Phi_{1}$. Ich verzichte daher darauf, die Tabelle aus Abschnitt 1.3 hier zu reproduzieren. Sie behält bis auf die Zeile, die den Steuersatz betrifft, Gültigkeit.

Unterschiede ergeben sich durch $\Phi_{2}$, wenn $t$ sich ändert. Die Differentiation von (2.3) ergibt

$$
\frac{\partial H^{*}}{\partial t}=-\Delta^{-1}\left(q U^{\prime}(G)+q U^{\prime \prime}(G) t\left(H^{*}-Y\right)+p U^{\prime \prime}(P) \beta Y\right)
$$

wobei $\Delta=q U^{\prime \prime}(G) t^{2}+p U^{\prime \prime}(P) \beta^{2}<0$ ist. Die Hypothese einer a.a.R. hilft nicht, den Zähler zu bestimmen, da die Umformung von (2.4) mit Hilfe von (2.3) nur zu

$$
\left.\frac{\partial H^{*}}{\partial t}=-\Delta^{-1} q U^{\prime}(G)\left(1+R_{a}(G) t\left(Y-H^{*}\right)-R_{a}(P) t Y\right)\right) \gtreqless 0
$$

führt. Auch die Reaktion der hinterzogenen Steuer $S^{*}=t H^{*}$ läßt sich im allgemeinen nicht vorhersagen:

$$
\frac{\partial S^{*}}{\partial t}=-\Delta^{-1} q U^{\prime}(G)\left(1+R_{a}(G) t Y-R_{a}(P)\left(t Y-\beta / t S^{*}\right)\right) \gtreqless 0
$$

Mit $t$ sinkt das sichere Einkommen $(1-t) Y$, so daß der Stpfl. ceteris paribus weniger hinterziehen wird. Auf der anderen Seite fördert der sinkende Preis der Hinterziehung $\beta / t$ den Steuerbetrug (Allingham/Sandmo, 1972, S. 330). Diese Erklärung der unbestimmten Ergebnisse soll der nächste Abschnitt mit Hilfe des dualen Ansatzes der Haushaltstheorie überprüfen. 


\section{Substitutions- und Einkommenseffekt der Besteuerung}

Die Wirkung des Steuersatzes auf das hinterzogene Einkommen und die hinterzogene Steuer unter der Strafe $\Phi_{2}$ wird im folgenden mit Hilfe einer Hicks-SlutskyGleichung untersucht. Zum Vergleich komme ich anschließend auf die Strafe $\boldsymbol{\Phi}_{\mathbf{1}}$ zurück, für die ebenfalls die entsprechende Zerlegung der "Nachfrageänderung" abgeleitet wird.

In der mikroökonomischen Haushaltstheorie teilt ein Haushalt sein Einkommen auf verschiedene Konsumgüter auf. Entscheidungsvariable und Argumente seiner Nutzenfunktion sind die Mengen der einzelnen Güter. Die Wirkung einer Preisänderung eines Gutes auf die nachgefragte Menge dieses Gutes zerlegt die Hicks-Slutsky-Gleichung in einen Substitutions- und einen Einkommenseffekt. Der Substitutions- oder Preiseffekt z.B. gibt an, wie sich die nachgefragte Menge eines Gutes ändert, wenn dessen Preis steigt und das Einkommen des Haushalts (hypothetisch) soweit erhöht wird, daß sein Nutzenniveau konstant bleibt (siehe etwa Varian, 1984, S. 130 f.). Diese Definition möchte ich verallgemeinern: Der Substitutionseffekt gibt an, wie ein Individuum eine bestimmte Entscheidungsvariable ändert, wenn ein Parameter seines Entscheidungsproblems variiert und sein Nutzen durch einen Einkommenstransfer auf dem Ausgangsniveau festgehalten wird. Den Einkommenseffekt kann man entsprechend verallgemeinern. Diese Definition von Einkommens- und Substitutionseffekt wird in dieser Arbeit verwendet. Sie werden konkret auf die Entscheidungsvariablen $H$ und $S=t H$ bezogen, die die Verteilung des Einkommens nach Steuer und Strafe auf die zwei Zustände "entdeckt" und "nicht entdeckt" steuern, nicht aber auf die Argumente der Erwartungsnutzenfunktion (die "Konsumgüter") $P$ und $G$. Wenn anders als in der Haushaltstheorie Entscheidungsvariable und Argumente der Nutzenfunktion auseinanderfallen, sollte man angeben, auf welche Größen Einkommens- und Substitutionseffekt bezogen werden. Bis auf zwei Ausnahmen (Sandmo, 1981, S. 272 - 274, Wiegard, 1984, S. 132) fehlt diese Angabe und eine entsprechende Hicks-Slutsky-Gleichung in der Literatur zur Steuerhinterziehung, obwohl bei der Interpretation der Modellergebnisse immer wieder von Einkommens- und Substitutionseffekt gesprochen wird.

Um Einkommens- und Substitutionseffekt formal zu definieren und zu berechnen ist das "duale Problem" des Stpfl. zu lösen. Dazu wir die Steuerfunktion durch einen Betrag $a$ ergänzt, den der Haushalt von der Steuerschuld abziehen kann. $G$ und $P$ lauten nun

$$
\begin{aligned}
& G=(1-t) Y+a+t H \\
& P=(1-t) Y+a-\beta H
\end{aligned}
$$

Eine Erhöhung des Transfers $a$ verschiebt die Budgetgerade im $P$ - $G$-Diagramm 
parallel nach oben. Das duale Problem lautet nun: Minimiere den Steuerabzug (Transfer) $a$ unter der Beschränkung eines gegebenen Erwartungsnutzens durch geeignete Wahl von $a$ und $H$. Bezeichnen wir die entsprechende Lagrange-Funktion mit $L$ und den Multiplikator der Nebenbedingung mit $\mu$, lautet das Minimierungsproblem:

$$
\min _{a, H, \mu} L=a+\mu(q U(G)+p U(P)-\bar{E})
$$

wobei $G$ und $P$ aus (2.5) und (2.6) eingesetzt werden. Die notwendigen Bedingungen erster Ordnung sind

$$
\begin{gathered}
L_{a}=1+\mu\left(q U^{\prime}(G)+p U^{\prime}(P)\right)=0 \\
L_{H}=\mu\left(q U^{\prime}(G) t-p U^{\prime}(P) \beta\right)=0 \\
L_{\mu}=q U(G)+p U(P)-\bar{E}=0
\end{gathered}
$$

Dieses Gleichungssystem bestimmt die Funktionen

$$
\begin{gathered}
H^{+}=H^{+}(p, \beta, t, Y, \bar{E})=H^{+}(\cdot, t, \bar{E}) \\
a^{+}=a^{+}(p, \beta, t, Y, \bar{E})=a^{+}(\cdot, t, \bar{E})
\end{gathered}
$$

$a^{+}$ist die "Ausgabenfunktion". Sie gibt den kleinsten Transfer $a$ an, mit dem der Stpfl. einen vorgegebenen Erwartungsnutzen $\bar{E}$ erreichen kann. $H^{+}$ist die "einkommenskompensierte. Nachfrage" nach dem hinterzogenen Einkommen: Für gegebene Werte von $t$ und $\bar{E}$ wählt der Stpfl. $H^{+}$.

$H^{*}$ ist die Lösung des primalen Problems: Maximiere den Erwartungsnutzen durch Wahl von $H$ bei gegebenem Transfer $a$. Zwischen $H^{*}$ und $H^{+}$gibt es eine Beziehung, die sich in folgender Identität ausdrücken läßt (vgl. Cook, 1972, Varian, 1984, S. 126, Sandmo, 1981, S. 272):

$$
H^{*}\left(\cdot, t, a^{+}(\cdot, t, \bar{E})\right) \equiv H^{+}(\cdot, t, \bar{E})
$$

Die normale (nicht kompensierte) Nachfrage bei einem Transfer $a^{+}$, der es ermöglichen würde, das Nutzenniveau $\bar{E}$ gerade zu erreichen, ist gleich der durch $a^{+}$kompensierten Nachfrage für den vorgegebenen Nutzen $\bar{E}$. Differenziert man diese Identität nach $t$, erhält man

$$
\frac{\partial H^{*}}{\partial t}=\frac{\partial H^{+}}{\partial t}-\frac{\partial a^{+}}{\partial t} \frac{\partial H^{*}}{\partial a}
$$

Der erste Term auf der rechten Seite ist der Substitutionseffekt. Er gibt an, wie sich das hinterzogene Einkommen ändert, wenn $t$ erhöht und $a$ dabei so angepaßt 
wird, daß der Haushalt auf seinem ursprünglichen Nutzenniveau verbleibt ${ }^{3,}{ }^{4}$. Der zweite Term (inclusive des Minuszeichens) ist der Einkommenseffekt. Er gibt an, wie $H^{*}$ reagiert, wenn man dem Haushalt durch Senkung von $a$ gerade das Einkommen nimmt, das ihm zwecks Kalkulation des Substitutionseffektes gegeben worden ist.

Im folgenden werden diese beiden Effekte berechnet. Um die Änderung des einkommenskompensierten hinterzogenen Einkommens zu bestimmen, wird das Gleichungssystem (2.7) - (2.9) implizit differenziert:

$$
\left(\begin{array}{ccc}
L_{a a} & L_{a H} & L_{a \mu} \\
L_{H a} & L_{H H} & 0 \\
L_{\mu a} & 0 & 0
\end{array}\right)\left(\begin{array}{c}
\partial a / \partial t \\
\partial H / \partial t \\
\partial \mu / \partial t
\end{array}\right)=\left(\begin{array}{c}
-L_{a t} \\
-L_{H t} \\
-L_{\mu t}
\end{array}\right)
$$

wobei

$$
\begin{aligned}
& L_{a a}=\mu\left(q U^{\prime \prime}(G)+p U^{\prime \prime}(P)\right)>0 \\
& L_{a \mu}=q U^{\prime}(G)+p U^{\prime}(P)>0 \\
& L_{H a}=\mu\left(q U^{\prime \prime}(G) t-p U^{\prime \prime}(P) \beta\right) \\
& L_{H H}=\mu\left(q U^{\prime \prime}(G) t^{2}+p U^{\prime \prime}(P) \beta^{2}\right)>0 \\
& L_{a t}=\mu\left(-q U^{\prime \prime}(G)(Y-H)-p U^{\prime \prime}(P) Y\right) \\
& L_{H t}=\mu\left(q U^{\prime}(G)-q U^{\prime \prime}(G) t(Y-H)+p U^{\prime \prime}(P) \beta Y\right) \\
& L_{\mu t}=-q U^{\prime}(G)(Y-H)-p U^{\prime}(P) Y<0
\end{aligned}
$$

sind. Die hochgestellten Plus-Zeichen an $a, H$ und $\mu$ sind weggelassen. Die zwei Stellen $L_{\mu H}$ sind wegen (2.8) gleich null. Die Vorzeichen einiger Elemente hängen von der Tatsache $\mu<0 \mathrm{ab}$.

\footnotetext{
${ }^{3}$ Statt der Hicks-Kompensation könnte man auch die Slutsky-Kompensation verwenden: $a$ wird so weit erhöht, daß der Stpfl. die Nettoeinkommen $G$ und $P$ der Ausgangslage erreichen kann. Für marginale Änderungen der Parameter erhält man auf beiden Wegen den gleichen Substitutionseffekt und folglich auch identische Einkommenseffekte (vgl. dazu Hatta/Wilke, 1982).

${ }^{4}$ Man könnte den Haushalt auch durch Erhöhung des exogenen Einkommens $Y$ kompensieren, um den Substitutionseffekt zu berechnen. In bezug auf $\mathrm{H}^{+}$ergeben sich dadurch keine Unterschiede. Wenn $Y$ jedoch geändert wird, kann man wegen $D^{+}=Y^{+}-H^{+}$von dem Effekt einer höheren Steuer auf $\mathrm{H}^{+}$nicht unmittelbar auf die Änderung von $D^{+}$schließen.
} 
Der Substitutionseffekt einer höheren Steuer ist

$$
\frac{\partial H^{+}}{\partial t}=\frac{-L_{H a} L_{\mu t}+L_{H t} L_{\mu a}}{-L_{H H} L_{a \mu}}
$$

Rechnet man weiter, ergibt sich

$$
\frac{\partial H^{+}}{\partial t}=\mu \frac{q U^{\prime}(G) L_{\mu a}+q U^{\prime \prime}(G) t H p U^{\prime}(P)+p U^{\prime \prime}(P) \beta H q U^{\prime}(G)}{-L_{H H} L_{a \mu}} \gtreqless 0
$$

Der Nenner ist negativ. Der Zähler läßt sich nicht bestimmen. Der Substitutionseffekt der höheren Steuer auf das hinterzogene Einkommen ist daher unbestimmt. Wegen der Konstanz von $Y$ gilt das auch für das einkommenskompensierte deklarierte Einkommen $D^{+}$.

Berechnen wir den Einkommenseffekt. Es gilt

$$
-\frac{\partial a^{+}}{\partial t}=\frac{L_{\mu t}}{L_{\mu a}}<0
$$

Aus der Bedingung erster Ordnung für das primale Problem - maximiere den Erwartungsnutzen für einen gegebenen Transfer $a-$ folgt

$$
\frac{\partial H^{*}}{\partial a}=\Delta^{-1} q U^{\prime}(G) t\left(R_{a}(G)-R_{a}(P)\right)>0
$$

wobei $\Delta=q U^{\prime \prime}(G) t^{2}+p U^{\prime \prime}(P) \beta^{2}<0$ ist. Faßt man beide Ausdrücke zusammen, erhält man einen negativen Einkommenseffekt.

Der Substitutionseffekt ist jedoch wie gesehen nicht eindeutig. Der Gesamteffekt der höheren Steuer auf das hinterzogene Einkommen ist damit ebenfalls nicht eindeutig. Dieses Ergebnis läßt sich mit Hilfe von Abbildung 2.1 verdeutlichen. Die Budgetgerade

$$
P=(1-t) Y+a-\frac{\beta}{t}(G-(1-t) Y-a)
$$

ist als $N M$ eingezeichnet. Eine Erhöhung von $t_{1}$ auf $t_{2}$ verschiebt $N M$ nach $A B$. Das neue Optimum ist $O_{2}$. Mit $a$ verschiebt sich $A B$ parallel nach oben. Wird der Transfer so weit erhöht, daß der Haushalt gerade wieder die ursprüngliche Indifferenzkurve erreicht, erhält man den Substitutionseffekt. Bei der Bewegung von $O_{1}$ nach $O_{3}$ steigt die hinterzogene Steuer, da der Preis der Hinterziehung einer DM Steuern $\beta / t$ sinkt: Der horizontale Abstand $O_{3} F$ ist größer als der horizontale Abstand $O_{1} N$. Daraus folgt jedoch nicht notwendigerweise, daß das hinterzogene Einkommen steigt (bzw. das deklarierte Einkommen sinkt).

$$
\frac{\partial\left(t H^{+}\right)}{\partial t}=H^{+}+t \frac{\partial H^{+}}{\partial t}>0
$$


Abbildung 2.1: Substitutions- und Einkommenseffekt der Besteuerung

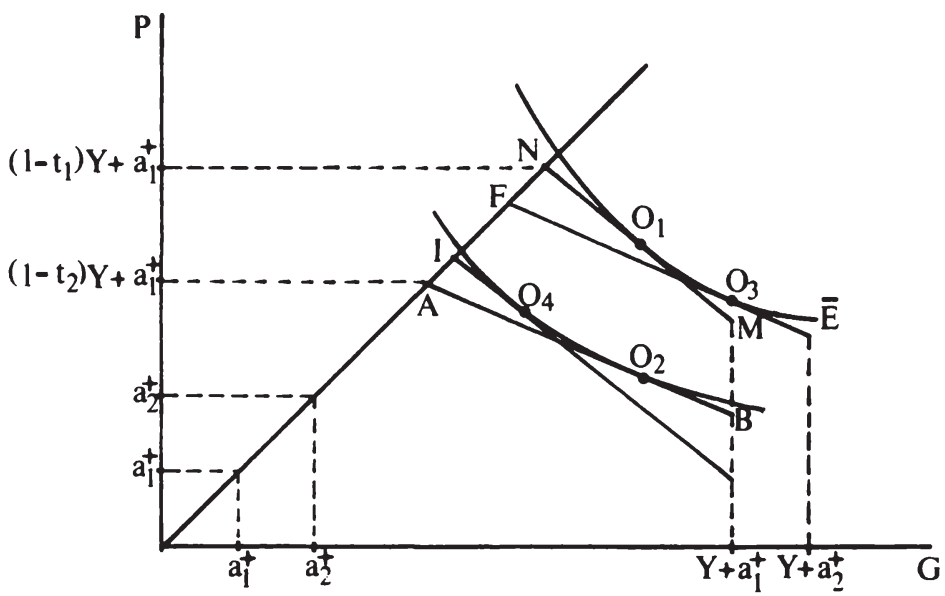

impliziert nicht $\partial H^{+} / \partial t>0$. Der Substitutionseffekt in bezug auf das hinterzogene Einkommen ist unbestimmt. Verwendet man jedoch statt $H$ die verkürzte Steuer $S=t H$ als Entscheidungsvariable, ergibt sich $\partial S^{+} / \partial t>0$, wie soeben anhand der Abbildung gezeigt worden ist. Der Einkommenseffekt - die Bewegung von $O_{1}$ nach $O_{4}$ - ist dagegen negativ, wenn man eine a.a.R. annimmt, und zwar unabhängig davon, ob man ihn auf $H$ oder $S$ bezieht. Der Haushalt hätte in Punkt $I$ ein geringeres sicheres Einkommen als in $N$. Wenn seine Bereitschaft, ein Risiko einzugehen, mit einem geringeren Einkommen $(1-t) Y+a$ sinkt, hinterzieht er in $\mathrm{O}_{4}$ weniger als in $O_{1}$.

Allingham/Sandmo (1972, S. 330) sprechen von einem positiven Substitutionseffekt der Steuer auf das hinterzogene Einkommen. Dies ist kein Widerspruch zu dem gerade abgeleiteten Ergebnis. Sie nehmen anders als hier an, daß der Stpfl. die hinterzogene Steuer nicht nachzahlen muß, falls er entdeckt wird. Er zahlt lediglich die Geldstrafe $\beta H$. Entsprechend ist (vgl. Allingham/Sandmo, 1972, S. 325)

$$
P=Y-t(Y-H)-\beta H=(1-t) Y-(\beta-t) H
$$

Die endgültige Steuerersparnis mindert in dieser Formulierung die Geldstrafe. Es muß hier $\beta>t$ angenommen werden, da der Stpfl. sonst kein Risiko trägt.

Mit Hilfe des oben vorgeführten Verfahrens kann man die Hicks-Slutsky-Gleichung für den Fall ohne die Nachzahlung der verkürzten Steuer berechnen. 
Sie lautet (vgl. Allingham/Sandmo, 1972, S. 329)

$$
\begin{aligned}
\frac{\partial H^{*}}{\partial t}= & -\Delta^{-1}\left(q U^{\prime}(G)+p U^{\prime}(P)\right) \\
& -\Delta^{-1}(Y-H) t q U^{\prime}(G)\left(R_{a}(G)-R_{a}(P)\right) \gtreqless 0
\end{aligned}
$$

wobei $\Delta=q U^{\prime \prime}(G) t^{2}+p U^{\prime \prime}(P)(\beta-t)^{2}$ ist. Der erste Term - der Substitutionseffekt - ist positiv. Im Unterschied zu dem zuerst untersuchten Fall mit einer Nachversteuerung erhöht der Stpfl. bei gegebenem Erwartungsnutzen das hinterzogene Einkommen. Der Verzicht auf die Nachzahlung verkürzter Steuern stimuliert in diesem Sinne den Steuerbetrug. Der Einkommenseffekt ist jedoch negativ. Der Gesamteffekt ist wieder nicht eindeutig.

Zum Vergleich komme ich jetzt auf die Wirkung der Besteuerung unter der Strafe $\Phi_{1}$ zurück. Yitzhaki (1974) hat gezeigt, daß der Einfluß der proportionalen Steuer auf das versteuerte Einkommen (bzw. $H^{*}$ ) eindeutig ist, wenn statt $\Phi_{2}$ die Strafe $\Phi_{1}$ verwendet wird. Seine Erklärung lautet, mit $\Phi_{1}$ "there is no substitution effect and we are left with a pure income effect" (Yitzhaki, 1974, S. 202). In der Literatur wird diese Behauptung oft wiederholt (siehe z.B. Gordon, 1989, S. 798, Cowell/Gordon, 1988, S. 306, Koskela, 1983, S. 130). Überprüfen wir sie mit Hilfe der Hicks-Slutsky-Gleichung ${ }^{5}$.

Diese lautet hier wieder ${ }^{6}$

$$
\frac{\partial H^{*}}{\partial t}=\frac{\partial H^{+}}{\partial t}-\frac{\partial a^{+}}{\partial t} \frac{\partial H^{*}}{\partial a}
$$

Die Erhöhung der Steuer verschiebt die Budetgerade im $P$ - $G$-Diagramm parallel nach unten. Die Kompensation über den Transfer $a$ verschiebt die Beschränkung parallel nach oben - und zwar zurück in die Ausgangslage. Es folgt, daß die hinterzogene Steuer $t H^{+}$konstant bleibt. Aus $\partial\left(t H^{+}\right) / \partial t=0$ ergibt sich

$$
\frac{\partial H^{+}}{\partial t}=-\frac{H^{+}}{t}
$$

Der Substitutionseffekt auf das hinterzogene (deklarierte) Einkommen ist nun negativ (positiv).

Die notwendige Kompensation der höheren Steuerbelastung muß man nicht aus dem dualen Problem ableiten. Es gibt einen zweiten Weg. Setzt man $H^{*}(\cdot, t, a)$ in die Zielfunktion des primalen Problems $q U(G)+p U(P)$ ein, erhält man die

\footnotetext{
${ }^{5}$ Die Unterscheidung zweier Fälle, je nachdem der Stpfl. hinterzogene Steuern nachzahlen muß oder nicht, spielt keine Rolle, wenn die Strafe ein Vielfaches der verkürzten Steuer ist. Siehe Abschnitt 1.2, Fußnote 6.

${ }^{6}$ Alle Variablen beziehen sich jetzt auf die Strafe $\Phi_{1}$. Die entsprechende Indizierung wird wie vereinbart unterdrückt.
} 
indirekte Erwartungsnutzenfunktion $E^{i}\left(\cdot, t, a, H^{*}(\cdot, t, a)\right)$. Da die Ausgabenfunktion $a^{+}(\cdot, t, \bar{E})$ die Inverse der indirekten Nutzenfunktion ist, ist die Ableitung der Ausgabenfunktion nach $t$ gleich der Grenzrate der Substitution der indirekten Nutzenfunktion zwischen $t$ und $a$ (vgl. Sandmo, 1981, S. 271).

$$
\frac{\partial a^{+}}{\partial t}=-\frac{\partial E^{i} / \partial t}{\partial E^{i} / \partial a}=Y-\frac{q U^{\prime}(G)-p U^{\prime}(P) \alpha}{q U^{\prime}(G)+p U^{\prime}(P)} H=Y
$$

wobei das Enveloppen-Theorem angewandt (Varian, 1984, S. 328) und die Optimalitätsbedingung des dualen Problems $q U^{\prime}(G)=p U^{\prime}(P) \alpha$ ausgenutzt wurde. Die Änderung von $a^{+}$ist leicht zu verstehen. Sie hält das sichere Einkommen nach Steuer $(1-t) Y+a$ konstant.

Weiter gilt

$$
\frac{\partial H^{*}}{\partial a}=\Delta^{-1} q U^{\prime}(G)\left(R_{a}(G)-R_{a}(P)\right)>0
$$

wobei $\Delta<0$ die entsprechende Determinante ist. Folglich ist der Einkommenseffekt $-Y \partial H^{*} / \partial a$ negativ. Der Gesamteffekt der Besteuerung setzt sich damit aus zwei negativen Teileffekten zusammen. Nur wenn die hinterzogene Steuer die Variable ist, auf die Substitutions- und Einkommenseffekt bezogen werden, stimmt die Aussage von Yitzhaki (1974), der erste sei null und der zweite negativ (vgl. Yitzhaki, 1987, S. 127). Der Fehler liegt somit darin, daß komparativ-statische Ergebnisse bezüglich der Variablen $H$ präsentiert werden, die Interpretation $H$ jedoch stillschweigend durch $t H$ ersetzt.

\section{Die deutsche Strafe}

Die Strafe nach deutschem Recht kann aus $\S 40$ Strafgesetzbuch abgeleitet werden (vgl. Frost, 1986, S. 7 und 23). Nach $\S 40$ StGB werden unabhängig voneinander die Zahl der Tagessätze und die Höhe eines Tagessatzes festgelegt. Die Multiplikation beider Größen ergibt die Geldstrafe. Die Höhe eines Tagessatzes ist das Nettoeinkommen, das der Stpfl. an einem Tag im Jahr durchschnittlich verdient (§ 40 Abs. 2 StGB). Die Anzahl der Tagessätze hängt nach $\S 46$ Abs. 2 StGB von den "verschuldeten Auswirkungen der Tat" ab, die bei dem hier betrachteten Vergehen gleich der Höhe der hinterzogenen Steuern sind (vgl. Dreher/Tröndle, 1986, Anm. $23 \mathrm{zu} \S 46$ StGB, Mösbauer, 1989, S. 71). Im einfachsten Fall werden die hinterzogenen Steuern mit einer Konstanten $\zeta$ multipliziert, um die Anzahl der Tagessätze zu erhalten. Die "deutsche Strafe" ist damit gleich

$$
\Phi_{3}=\frac{(1-t) Y}{360} \times \zeta t H=\gamma(1-t) Y t H
$$

wobei $\gamma=\zeta / 360$ ist. 
Ersetzen wir die Strafe in dem Grundmodell durch $\Phi_{3}$. Dann gilt ${ }^{7}$

$$
P=(1-t) Y-\gamma(1-t) Y t H
$$

Der Haushalt löst das Problem

$$
\begin{array}{cl}
\max _{H} & q U(G)+p U(P) \\
\text { u.d.N. } & 0 \leqq H \leqq Y
\end{array}
$$

wobei $G$ und $P$ durch (2.2) und (2.10) definiert sind. Die Bedingung erster Ordnung für eine innere Lösung ist

$$
q U^{\prime}(G)-p U^{\prime}(P) \gamma(1-t) Y=0
$$

Das hinterzogene Einkommen $H^{*}$ ist positiv, wenn $q-p \gamma(1-t) Y>0$ gilt. Die Bedingung für $H^{*}>0$ ist nun auch noch von $Y$ abhängig. Eine diskrete (große) Erhöhung des Einkommens kann einen Haushalt, der in der Ausgangslage hinterzieht, dazu veranlassen, das tatsächliche Einkommen zu deklarieren. Mit der "deutschen Strafe" wird der Stpfl. (bei konstanten $p, t, \gamma$ ) bei sukzessiver Erhöhung des Einkommens immer an einen Punkt kommen, an dem er steuerehrlich ist.

Aus (2.2) und (2.10) erhalten wir

$$
P=(1-t) Y-\gamma(1-t) Y[G-(1-t) Y]
$$

Aus dieser Gleichung läßt sich schließen, daß die Entdeckungswahrscheinlichkeit und der Strafsatz $\gamma$ die üblichen Effekte haben. Welche Wirkungen haben jedoch die Besteuerung und eine marginale Erhöhung des Einkommens mit der deutschen Strafe? Betrachten wir (2.11). Eine höhere Steuerbelastung senkt das sichere Nettoeinkommen $(1-t) Y$; gleichzeitig vermindert sie den Preis der Steuerhinterziehung $\gamma(1-t) Y$, da der Tagessatz negativ von $t$ abhängt. Ein höheres exogenes Einkommen wirkt in die entgegengesetzte Richtung: $(1-t) Y$ und $\gamma(1-t) Y$ steigen. In Abbildung 2.2 verschiebt sich $N M$ mit sukzessiv höheren Steuersätzen $t_{2}>t_{1}, t_{3}>t_{2}$ nach $C D$ und $E F$. Mit höheren Einkommen $Y_{2}>Y_{1}, Y_{3}>Y_{2}$ verschiebt sich $N M$ nach $L I$ und $J K$. Es ist daher zu vermuten, daß sowohl die Steuer als auch das Einkommen eine unbestimmte Wirkung auf das hinterzogene Einkommen haben. Diese Vermutung läßt sich bestätigen. Ich zeige das für den Parameter $Y$.

$$
\frac{\partial H^{*}}{\partial Y}=-\Delta^{-1}(1-t)\left\{q U^{\prime}(G)\left[-R_{a}(G)+R_{a}(P)\left(1-\gamma t H^{*}\right)\right]-p U^{\prime}(P) \gamma\right\}
$$

\footnotetext{
${ }^{7}$ In (2.10) ist die Nachzahlung der verkürzten Steuer angenommen. Frost (1986) erläßt dem Steuersünder in seiner Modellierung deutschen Rechts die Nachzahlung. Diese ist jedoch nach der Abgabenordnung eine Konsequenz der Steuerhinterziehung: $\S 71$ AO bestimmt, daß der Steuerhinterzieher für die verkürzten Steuern haftet.
} 
Abbildung 2.2: Die Strafe nach deutschem Recht

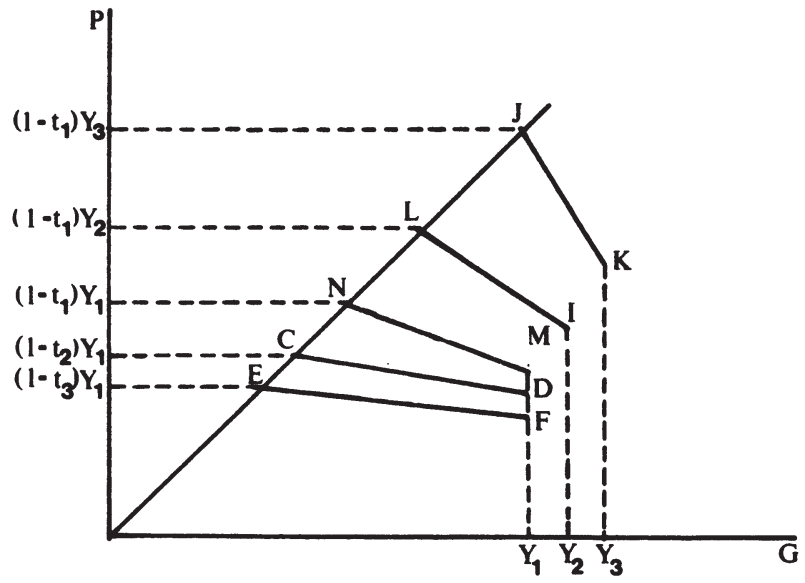

wobei $\Delta=q U^{\prime \prime}(G) t+p U^{\prime \prime}(P)(\gamma(1-t) Y)^{2} t<0$ ist. Der Term $1-\gamma t H^{*}$ läßt sich bestimmen. Wenn ein Stpfl. auch für den Fall einer Prüfung einen positiven Konsum sicherstellen will (d.h. wenn $\lim _{C \rightarrow 0} U^{\prime}(C)=\infty$ angenommen wird), muß

$$
(1-t) Y>\gamma(1-t) Y t H^{*} \quad \Leftrightarrow \quad 1>\gamma t H^{*}
$$

gelten. Die eckige Klammer in (2.12) enthält damit einen positiven und einen negativen Ausdruck und es gilt $\partial H^{*} / \partial Y \gtreqless 0$. Auch über $t H^{*}, D^{*}$ und das Steueraufkommen kann man im allgemeinen nichts aussagen, wenn $Y$ variiert. Entsprechende Aussagen gelten für $t$. In beiden Fällen setzt sich die Verhaltensänderung des Stpfl. aus entgegengesetzten Substitutions- und Einkommenseffekten zusammen. Es soll darauf verzichtet werden, diese Zerlegung explizit anzugeben.

\section{Strafen oder Kontrollen als Mittel gegen die Steuerhinterziehung?}

Das Grundmodell liefert durchgehend bestimmte Ergebnisse, wenn man Arrows Hypothese über den Verlauf der absoluten Risikoaversion folgt. Der Ersatz der Strafe $\Phi_{1}$ durch $\Phi_{2}$ und weiter durch $\Phi_{3}$ führt zu teilweise unbestimmten Resultaten: Mit $\Phi_{2}$ ist die Wirkung der Besteuerung auf die Hinterziehung, mit $\Phi_{3}$ zusätzlich noch der Einfluß des Bruttoeinkommens nicht bestimmbar. Neben diesen Unterschieden fällt eine gemeinsame Eigenschaft dieser drei Strafen auf. Die Entdeckungswahrscheinlichkeit und der jeweilige Strafsatz $(\alpha, \beta$ und $\gamma)$ haben stets die gleiche Wirkung: Strafen und Kontrollen sind wirksame Mittel, um dem Steuerbetrug zu begegnen. 
Abbildung 2.3: Entdeckungswahrscheinlichkeit und Strafe bei konstantem erwarteten Ertrag der Hinterziehung

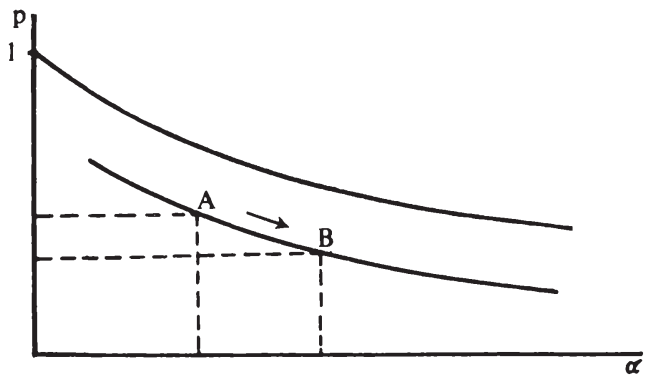

Diese zentrale Botschaft des bisher analysierten Modells wirft zwei Fragen auf. Gilt die Aussage auch in komplizierteren Modellen mit mehr als einer Entscheidungsvariablen? Sind Kontrollen oder Strafen das wirksamere Mittel gegen die Steuerhinterziehung? Die erste Frage wird in Abschnitt 2.2 und in den folgenden Kapiteln behandelt. Die Antwort auf die zweite Frage, die Christiansen (1979) anbietet, wird an dieser Stelle wiedergegeben.

Ist eine hohe Kontrollwahrscheinlichkeit gekoppelt mit einer geringen Strafe eher geeignet, dem Steuerbetrug zu begegnen, als eine hohe Strafe zusammen mit einer geringen Kontrollwahrscheinlichkeit (Christiansen, 1979, S. 389)? Ist also z.B. in Abbildung 2.3 die Kombination $A$ von $\alpha$ und $p$ oder die Kombination $B$ mit einer geringeren Steuerhinterziehung verbunden? Christiansen beantwortet diese Frage, indem er die Bewegung in Richtung höherer Strafen und geringerer Entdeckungswahrscheinlichkeiten entlang der unteren in Abbildung 2.3 gezeichneten Kurve analysiert. Der eingezeichnete Zusammenhang beruht auf der Verwendung von $\Phi_{1}$ und der Forderung, daß der erwartete durchschnittliche Ertrag der Steuerhinterziehung konstant gleich $k(0<k<1)$ bleibt, während $\alpha$ steigt und $p$ sinkt ${ }^{8}$ :

$$
(1-p)-p \alpha=k \quad \Leftrightarrow \quad p=\frac{1-k}{1+\alpha}
$$

Man kann natürlich auch einen anderen negativen Zusammenhang zwischen $p$ und $\alpha$ unterstellen. Der in (2.13) angenommene ist aber besonders interessant:

\footnotetext{
${ }^{8}$ Die obere Kurve in Abbildung 2.3 bildet die Gleichung $(1-p)-p \alpha=0$ ab. Nur bei Kombinationen von $p$ und $\alpha$ unterhalb dieser Kurve wird hinterzogen.
} 
Bei konstantem erwarteten Ertrag der Hinterziehung ändert ein risikoneutraler Stpfl. sein Verhalten nicht. Wie aber reagiert ein risikoaverser Haushalt auf einen "mean-preserving spread" der Verteilung des Ertrags der Hinterziehung (vgl. Rothschild/Stiglitz, 1970, S. 229, 1971, S. 66 und S. 72)?

Wenn $p$ von $\alpha$ abhängt, gilt

$$
\left.\frac{\partial H^{*}}{\partial \alpha}\right|_{q-p \alpha=k}=\frac{\partial H^{*}}{\partial \alpha}+\frac{\partial H^{*}}{\partial p} \frac{\partial p}{\partial \alpha}
$$

Aus (2.13) ergibt sich $\partial p / \partial \alpha=-p /(1+\alpha)$. Die partiellen Ableitungen von $H^{*}$ nach $p$ und $\alpha$ entnehme ich Abschnitt 1.4. Damit ist (vgl. Christiansen, 1979, S. 391)

$$
\begin{aligned}
\left.\frac{\partial H^{*}}{\partial \alpha}\right|_{q-p \alpha=k} & =\Delta^{-1}\left[p U^{\prime}(P)-p U^{\prime \prime}(P) \alpha t H^{*}-\left(U^{\prime}(G)+U^{\prime}(P) \alpha\right) \frac{p}{1+\alpha}\right] \\
& =\Delta^{-1}\left[\left(U^{\prime}(P)-U^{\prime}(G)\right) \frac{p}{1+\alpha}-p U^{\prime \prime}(P) \alpha t H^{*}\right]<0
\end{aligned}
$$

Für die Bestimmung des Vorzeichens ist die Risikoaversion per se ausreichend. Das Ergebnis lautet: "If the fine is increased, but the efforts to detect tax evaders are adjusted so as to keep the expected gain from tax evasion unaltered, risk averters will always reduce their tax evasion" (ebenda, S. 391). Die Hinterziehung sinkt bei der Bewegung von $A$ nach $B$ in Abbildung 2.3.

Dasselbe Resultat erhält man mit $\Phi_{3}$. Das ist nicht weiter überraschend, da der $\alpha$ entsprechende Multiplikator $\gamma(1-t) Y$ sich nur mit $\gamma$ ändert, solange $t$ und $Y$ nicht variiert werden. Die Strafe $\Phi_{2}$ läßt jedoch überraschenderweise unbestimmt, ob eine Reform in Richtung höherer Strafen und geringerer Intensität von Kontrollen die Hinterziehung reduziert.

Falls es Ziel des Staates sein sollte, die Steuerehrlichkeit der Bürger zu fördern, ist die steuerpolitische Empfehlung, die das Ergebnis von Christiansen nahelegt, offensichtlich - zumal dann, wenn es weniger Kosten verursacht, hohe Strafen zu verhängen, als die Steuerfahndung personell und materiell zu verstärken. Außerhalb des Modells gibt es jedoch Grenzen für die Politik, die Bestrafung auf Kosten der Kontrollen zu erhöhen: Die Strafe sollte in einer angemessenen Relation zu dem Vergehen stehen (vgl. Christiansen, 1979, S. 391). Die Steuersünder mit einer Wahrscheinlichkeit von (fast) null zu hängen, ist keine empfehlenswerte Maßnahme.

Zum Schluß dieses Abschnitts über alternative Formen der Geldstrafe noch ein Hinweis. Im weiteren Verlauf der Arbeit wird zumeist $\Phi_{1}$, teilweise auch $\Phi_{3}$ verwendet. Beide sind empirisch relevant, da sowohl in der Bundesrepublik als auch in den Vereinigten Staaten die Strafe von der hinterzogenen Steuer abhängig ist. Das deutet darauf hin, daß der Fiskus in erster Linie an die Steuerausfälle denkt. 
Das hinterzogene Einkommen an sich ist ihm gleichgültig. Mit umgekehrten Vorzeichen gilt dies für den Stpfl. Die Strafe $\Phi_{2}$ hat daher sozusagen die "falsche" Bemessungsgrundlage. Allingham/Sandmo war bei der Formulierung ihrer Strafe nicht klar, daß die hinterzogene Steuer die zentrale Größe in der Auseinandersetzung zwischen dem Stpfl. und der Finanzbehörde ist.

$\Phi_{1}$ werde ich $\Phi_{3}$ in der Regel vorziehen, da sie einfacher zu handhaben ist. $\Phi_{3}$ führt vielfach zu unbestimmten Ergebnissen. In einem interessanten Fall, der in Kapitel $4 \mathrm{zu}$ finden ist, haben sie jedoch die gleichen Wirkungen.

\subsubsection{Variable Entdeckungswahrscheinlichkeit}

Die Entdeckungswahrscheinlichkeit muß nicht konstant sein. Sie kann von Informationen abhängig sein, die die Finanzbehörde schon vor einer Prüfung über den Stpfl. besitzt. In einem Modell mit einer einzigen Veranlagungsperiode kennt die Behörde nur wenige Merkmale des Stpfl.: das deklarierte Einkommen und zusätzlich vielleicht seinen Beruf. Das tatsächliche oder das hinterzogene Einkommen kennt sie vor einer Prüfung annahmegemäß nicht, und $p$ kann daher keine Funktion von $Y$ oder $H$ sein (so aber Srinivasan, 1973, Yitzhaki, 1987).

Allingham/Sandmo (1972, S. 332 f.) nehmen an, daß die Entdeckungswahrscheinlichkeit mit dem deklarierten Einkommen $D=Y-H$ sinkt:

$$
\begin{aligned}
& p=p(D), \quad p^{\prime}<0, \quad p^{\prime \prime} \geqq 0 \\
& q=q(D)=1-p(D)
\end{aligned}
$$

Die Behörde hat eine gewisse Vorstellung über das durchschnittliche Einkommen, das in dem Beruf des Stpfl. verdient werden kann. Je mehr das deklarierte Einkommen von dem durchschnittlichen Einkommen nach unten (oben) abweicht, desto größer (geringer) wird die Entdeckungswahrscheinlichkeit. Bei einem sehr kleinen deklarierten Einkommen muß sich das Finanzamt schließlich fragen, wovon der Stpfl. denn leben will.

Die Erwartungsnutzenfunktion lautet nun:

$$
q(Y-H) U(G)+p(Y-H) U(P)
$$

Mit $H$ als Entscheidungsvariable und der Strafe $\Phi_{1}$ erhalten wir die Bedingungen erster und zweiter Ordnung für eine innere Lösung:

$$
\begin{aligned}
& q U^{\prime}(G) t-p U^{\prime}(P) \alpha t+p^{\prime}(U(G)-U(P))=0 \\
\Delta= & q U^{\prime \prime}(G) t^{2}+p U^{\prime \prime}(P) \alpha^{2} t^{2} \\
& +2 p^{\prime} t\left(U^{\prime}(G)+U^{\prime}(P) \alpha\right)-p^{\prime \prime}(U(G)-U(P))<0
\end{aligned}
$$


Im Vergleich zu der Optimumbedingung bei konstanter Entdeckungswahrscheinlichkeit enthält (2.14) einen zusätzlichen negativen Term: $p^{\prime}(U(G)-U(P))$ sind die Grenzkosten der Hinterziehung, die auf die erhöhte Kontrollwahrscheinlichkeit zurückzuführen sind.

Die Ergebnisse des Modells sind: Das hinterzogene Einkommen sinkt mit einem höheren Strafsatz $\alpha$. Eine Verschiebung der Funktion $p(Y-H)$ nach oben bewirkt ebenfalls eine geringere Hinterziehung. Dieses Resultat ergibt sich, wenn man die Entdeckungswahrscheinlichkeit als $\epsilon p(Y-H)$ oder $\epsilon+p(Y-H)$ schreibt und die Ableitung von $H^{*}$ nach $\epsilon$ berechnet. Die gleichen qualitativen Zusammenhänge erhält man, wenn statt $\Phi_{1}$ die Strafe $\Phi_{2}$ verhängt wird (Allingham/Sandmo, 1972, S. 332). Die partielle Ableitung des hinterzogenen Einkommens nach $Y$ ist

$$
\begin{aligned}
\frac{\partial H^{*}}{\partial Y}= & -\Delta^{-1}\left[p^{\prime}\left(U^{\prime}(G)-U^{\prime}(P)\right)(1-t)+p^{\prime \prime}(U(G)-U(P))\right. \\
& -p U^{\prime}(P) \alpha t(1-t)\left(R_{a}(G)-R_{a}(P)\right) \\
& \left.+p^{\prime}(U(G)-U(P))(1-t) R_{a}(G)-p^{\prime} t\left(U^{\prime}(G)+U^{\prime}(P) \alpha\right)\right] \gtreqless 0
\end{aligned}
$$

Bis auf den zweitletzten sind alle Summanden in der eckigen Klammer größergleich null. Es kann daher nicht ausgeschlossen werden, daß $H^{*}$ mit $Y$ sinkt! Bei gegebenem $p$ stimuliert ein höheres Einkommen zwar den Steuerbetrug. Wenn gleichzeitig das deklarierte Einkommen sinkt, steigt jedoch das variable $p$, so daß ein gegenläufiger Effekt entsteht.

Erhöht sich der Steuersatz, kann allein mit der Annahme einer a.a.R. nicht gesagt werden, ob das hinterzogene Einkommen sinkt, wenn $p$ von $Y-H$ abhängt. Ein höheres deklariertes Einkommen verringert die Entdeckungswahrscheinlichkeit, so daß ein zusätzlicher, gegenläufiger Effekt entsteht.

Wenn ein Stpfl. in mehr als einer Periode veranlagt wird, gelangt die Finanzbehörde zu weiteren Informationen, von denen sie eine Kontrolle abhängig machen kann. Naheliegend ist, daß sie die Steuererklärung für das gerade abgelaufene Jahr eher prüft, wenn sie den Stpfl. in der Vergangenheit schon einmal entdeckt hat. Auf diese Möglichkeit geht Kapitel 6 ein, das Modelle mit wiederholter Veranlagung behandelt.

\subsubsection{Progressiver Tarif}

Das Grundmodell der Steuerhinterziehung hat Pencavel (1979) um einen speziellen direkt progressiven und Koskela (1983) um einen indirekt progressiven Tarif erweitert. Die Frage ist jeweils, ob sich die Ergebnisse bei proportionalem Tarif verallgemeinern lassen. Auf den ersten Blick neigt man dazu, diese Frage zu verneinen. Denn Bamberg/Richter (1988) zufolge ist in Modellen mit Unsicherheit die Unbestimmtheit der Besteuerungswirkung die zu erwartende Eigenschaft eines 
progressiven Tarifs. Bei der Steuerhinterziehung ist dies jedoch nicht der Fall. Es wird im folgenden gezeigt, daß sich fast alle Ergebnisse des Grundmodells mit progressivem Tarif reproduzieren lassen. In dem Abschnitt zur indirekten Progression geht es dann um eine von Koskela (1983) vorgeschlagene Umgestaltung des Tarifs, die zu einer größeren Steuerehrlichkeit führt, ohne das Steueraufkommen zu berühren.

\section{Direkte Progression}

Pencavel (1979) arbeitet mit der Steuerbetragsfunktion $t B^{\sigma}$ der Bemessungsgrundlage $B>0$. Mit $\sigma>1$ hat man den interessierenden Fall eines mit $B$ steigenden marginalen Steuersatzes. Diese spezielle Form ist nicht notwendig für Pencavels Ergebnisse. Die allgemeine direkt progressive Steuer $T(B)$ mit den Eigenschaften (für $B>0$ )

$$
\begin{array}{r}
B>T(B)>0 \\
1>T^{\prime}(B)>0 \\
\\
T^{\prime \prime}(B)>0
\end{array}
$$

liefert dieselben Resultate.

Der Konsum des Stpfl. ist entweder

$$
G=Y-T(Y-H)=Y-T(Y)+[T(Y)-T(Y-H)]
$$

oder

$$
P=Y-T(Y)-\alpha[T(Y)-T(Y-H)]
$$

Die hinterzogene Steuer ist die Differenz zwischen der Steuer für das tatsächliche Einkommen und der aufgrund des deklarierten Einkommens veranlagten Steuer. Die verwendete Geldstrafe ist $\Phi_{1}$. Setzt man (2.15) in (2.16) ein, ergibt sich die Gerade

$$
P=Y-T(Y)-\alpha(G-Y+T(Y))
$$

Vergleichen wir sie mit der entsprechenden Budgetgeraden bei proportionalem Tarif, läßt sich schließen: Die hinterzogene Steuer sinkt mit $p$ und $\alpha$. Aus

$$
\frac{\partial\left[T(Y)-T\left(Y-H^{*}\right)\right]}{\partial p}=T^{\prime}\left(Y-H^{*}\right) \frac{\partial H^{*}}{\partial p}<0
$$

folgt, daß auch $H^{*}$ mit $p$ sinkt. Bezüglich $\alpha$ gilt die entsprechende Aussage.

Ein höheres Bruttoeinkommen bewirkt steigende hinterzogene Steuern, wenn eine a.a.R. unterstellt wird. Dies zeigt Abbildung 2.4 für eine Verdoppelung des Einkommens von $Y_{1}$ auf $Y_{2}=2 Y_{1}$. Die Budgetgerade verschiebt sich parallel nach 
Abbildung 2.4: Der Einfluß eines höheren Einkommens bei progressivem Tarif

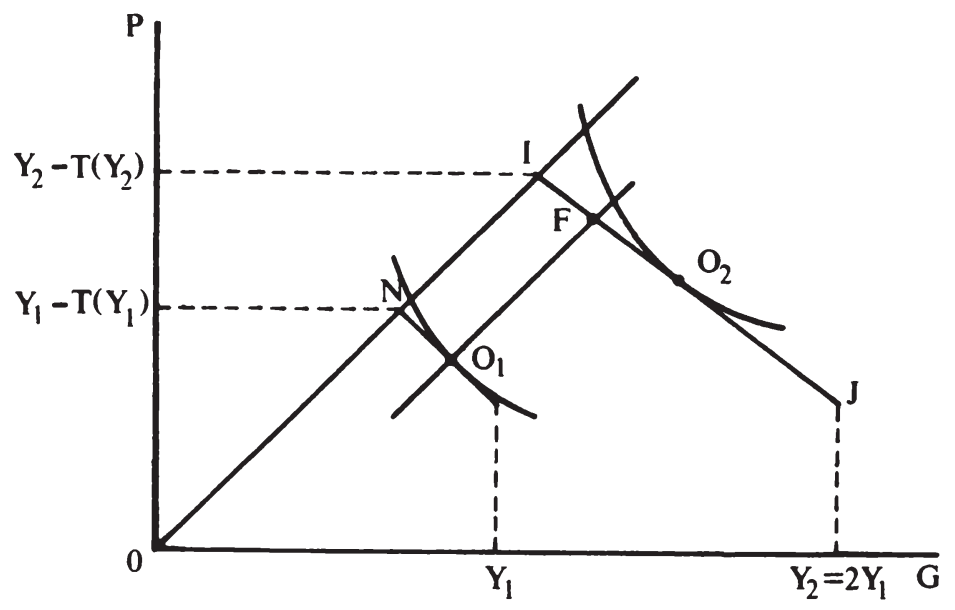

außen. Im Unterschied zu der entsprechenden Abbildung für einen proportionalen Tarif ist die Strecke $I 0$ auf der $45^{\circ}$-Linie infolge der direkten Progression nicht doppelt so lang wie die Strecke N0: Das (sichere) Nettoeinkommen $Y-T(Y)$ verdoppelt sich nicht, wenn das Bruttoeinkommen mit zwei multipliziert wird. Das ändert aber nichts daran, daß bei (mit $Y-T(Y)$ ) abnehmender absoluter Risikoaversion die von dem Stpfl. gewählte Position auf $I J$ rechts des Punktes $F$ liegt, in dem die durch $O_{1}$ verlaufende Parallele zu der $45^{\circ}$-Linie die Budgetgerade $I J$ schneidet. Die hinterzogene Steuer in $\mathrm{O}_{2}$ ist damit größer als in der Ausgangssituation. Daraus folgt aber nicht, daß $H^{*}$ gestiegen ist. Denn aus

$$
\frac{\partial\left[T(Y)-T\left(Y-H^{*}\right)\right]}{\partial Y}=T^{\prime}(Y)-T^{\prime}\left(Y-H^{*}\right)\left(1-\frac{\partial H^{*}}{\partial Y}\right)>0
$$

folgt nur

$$
\frac{\partial H^{*}}{\partial Y}>1-\frac{T^{\prime}(Y)}{T^{\prime}\left(Y-H^{*}\right)}
$$

Da die rechte Seite dieser Ungleichung kleiner null ist, kann man nicht sagen, ob $H^{*}$ mit $Y$ steigt oder sinkt. Bei steigendem marginalen Steuersatz kann die hinterzogene Steuer auch dann zunehmen, wenn das hinterzogene Einkommen abnimmt. Für die Reaktion des deklarierten Einkommens kann man aus $D=Y-H$ und 
(2.17) eine Obergrenze ableiten:

$$
\frac{\partial D^{*}}{\partial Y}<\frac{T^{\prime}(Y)}{T^{\prime}\left(Y-H^{*}\right)}<1
$$

Die Parameter $t$ und $\sigma$ des von Pencavel verwendeten Tarifs haben bei $T(B)$ keine Entsprechung. Beide Parameter bewirken ein steigendes deklariertes Einkommen (Pencavel, 1979, S. 120). Sie führen beide zu einem Anstieg des marginalen Steuersatzes $t \sigma B^{\sigma-1}$. Die Budgetgerade $P(G)$ verschiebt sich damit im $P-G$-Diagramm parallel nach innen.

Die Kombination von progressivem Tarif und der progressiven deutschen Strafe - für jeweils gleiche Vergehen zahlen Stpfl. mit höherem Einkommen größere Strafen - bringt im Vergleich zu der Analyse mit dem proportionalen Tarif keine neuen Ergebnisse. Auf die Betrachtung dieses Falls soll daher verzichtet werden.

\section{Indirekt progressiver Tarif}

Indirekt progressiv sind der von Koskela (1983) verwendete Tarif $t B-a$, bei dem eine Steuerermäßigung $a$ eingeräumt wird, und die Funktion $t(B-f)$, bei der von dem Einkommen ein Freibetrag $f$ absetzbar ist. Die Einführung der indirekten Progression führt nicht dazu, daß sich die Ergebnisse der komparativen Statik in bezug auf einzelne Parameter ändern. Interessanter ist die Frage, wie der Stpfl. auf eine Umgestaltung des Tarifs (d.h. eine simultane Änderung von $t$ und $a$ bzw. $t$ und $f$ ) reagiert, die den Progressionsgrad erhöht, aber das erwartete Steueraufkommen konstant läßt ${ }^{9}$. Das Maß für den Progressionsgrad ist hier der Grad der Änderung des Durchschnittsteuersatzes. Koskela (1983, S. 132 f.) zeigt: Erhöht der Staat $t$ und $a$, so daß das erwartete Steueraufkommen $A=t Y-a-(q-p \alpha) t H^{*}$ unverändert bleibt, sinkt das hinterzogene Einkommen, wenn man $\Phi_{1}$ verwendet und eine a.a.R. annimmt. Wenn es also Ziel des Staates wäre, die Bürger zu mehr Steuerehrlichkeit zu veranlassen, könnte er es kostenlos erreichen, da die vorgeschlagene Umgestaltung des Tarifs keine Steuerausfälle verursacht, andererseits den "repräsentativen" Steuerzahler nicht höher belastet und daher leicht zu rechtfertigen wäre. Diese Aussage ist natürlich dem Einwand ausgesetzt, daß die Stpfl. nicht alle gleich sind, aber dem gleichen Tarif unterliegen.

\subsection{Endogene Steuerbemessungsgrundlage}

Die Erweiterung des Modells um ein endogenes Einkommen hat eine einleuchtende Begründung. Zwischen der Steuerbelastung bzw. den Möglichkeiten, der

\footnotetext{
${ }^{9}$ Stiglitz (1969) untersucht diese Frage in einem Modell, in dem der Stpfl. keine Steuern hinterziehen, aber zwischen sicheren und unsicheren Kapitalanlagen wählen kann.
} 
Besteuerung zu entgehen, und der Entstehung des Einkommens gibt es langfristig im allgemeinen einen Zusammenhang. Ein Stpfl. wird bei seinen Entscheidungen über das Arbeitsangebot, die Ersparnis etc. die zu erwartende Steuerbelastung seines Einkommens berücksichtigen, und es fragt sich, wie dieser Zusammenhang die Steuerhinterziehung beeinflußt. In der kurzen Frist ist ein exogenes Einkommen dagegen eine sinnvolle Annahme: Das Einkommen der laufenden Periode ist zumeist auf Entscheidungen vergangener Perioden zurückzuführen - ganz deutlich ist dies bei Zinserträgen. Das Einkommen ändert sich daher nicht, wenn in der laufenden Periode finanzpolitische Parameter geändert werden.

Geht man von einer endogenen Steuerbemessungsgrundlage aus, ergeben sich entsprechend der Vielfalt der Steuerarten verschiedenen Modelle, die sich zum Teil deutlich voneinander unterscheiden. Dieser Abschnitt gibt einen Überblick zu den Arbeiten, die die Entstehung der Steuerbasis zusammen mit ihrer Deklaration gegenüber der Finanzbehörde analysieren. Er besteht zunächst aus einer Übersicht zu den vorhandenen Modellen. Anschließend wird exemplarisch auf die Arbeiten vom Marelli (1984), Pencavel (1979) und Cowell (1985) eingegangen. Der folgenden Frage soll dabei nicht ausgewichen werden: Was tragen diese Modelle zu einem besseren Verständnis der Besteuerungspraxis bei?

\subsubsection{Modelle der Steuerhinterziehung mit endogener Steuerbemessungsgrundlage}

Die Modelle mit endogener Steuerbasis unterteile ich in zwei Gruppen. Die einen behandeln steuerpflichtige Haushalte, die anderen Unternehmen. Innerhalb dieser beiden Gruppen wird nach der Steuerart differenziert. Unter der Steuerart sind in Tabelle 2.1 jeweils die Entscheidungsvariablen angegeben, die neben die Hinterziehung treten. Die meisten Modelle beschränken sich auf insgesamt zwei endogene Variable. Die Tabelle gibt zu jeder Steuer die wichtigsten Veröffentlichungen an. Zusätzlich wird auf Kapitel dieser Arbeit verwiesen.

Der Schwerpunkt der Literatur liegt bei der Hinterziehung von Lohneinkommen durch Haushalte. Das ist überraschend, da die Lohneinkommen der unselbständig Beschäftigten in vielen Ländern einer Quellensteuer unterliegen. Die Unternehmen führen einen Steuerabzug durch, so daß die Arbeitnehmer die Löhne nicht hinterziehen können. Das Modell von Pencavel (1979) und anderen wäre damit nur auf selbständig Beschäftigte anwendbar, da diese zur Einkommensteuer veranlagt werden. Viele Arbeitnehmer können jedoch in einem Unternehmern arbeiten, das die Lohnsteuer abführt, und/oder sie können "schwarz" arbeiten. Die Arbeit in der "Schattenwirtschaft" beinhaltet die Hinterziehung aller Abgaben (für Definitionen und Abgrenzungen der Schattenwirtschaft siehe Gaertner/Wenig, 1985, Marelli, 1987). Kann ein Arbeiter den Umfang der Schwarzarbeit kontinuierlich variieren, 
Tabelle 2.1: Modelle mit endogener Steuerbasis

\section{Haushalte}

Einkommensteuer

- Arbeitseinkommen
Andersen (1977)
Pencavel (1979)
Baldry (1979)
Sandmo (1981)
Wiegard (1984)
Cowell (1985)

- Zinseinkommen bzw. Ersparnis

Direkte Konsumsteuer

- Ersparnis

Kapitel 3 und 4

Kapitel 5

Unternehmen

Indirekte Steuern

- Umsatz

Marelli (1984)

Marelli/Martina (1988)

Virmani (1989)

Gewinnsteuer

- legale Beschäftigung und

Braulke/Sauermann (1986) Einsatz von Schwarzarbeit

- Güterangebot

Marelli/Martina (1988)

Lohnsteuer

- Beschäftigungshöhe

Yaniv (1988)

Braulke/Sauermann (1986) 
kann ein Modell mit einer offiziellen Beschäftigung und Schwarzarbeit dem Modell eines Stpfl. entsprechen, dessen Arbeitseinkommen nach dem Veranlagungsprinzip besteuert wird. Auf diese Entsprechung gehen ich unten kurz ein. Beide Ansätze beziehen sich auf Dauerthemen der wirtschaftspolitischen Diskussion: die Determinanten der Schwarzarbeit und die Steuerdisziplin der Selbständigen. In den letzten Jahren hat sich die Literatur steuerhinterziehenden Unternehmen zugewandt. Insgesamt gibt es bei der Steuerhinterziehung mit endogener Steuerbemessungsgrundlage noch einige Lücken, zu deren Verkleinerung diese Arbeit durch die Analyse der Hinterziehung von Zinseinkommen und der Steuerhinterziehung unter einer direkten Konsumsteuer beitragen soll.

\subsubsection{Umsatzsteuerhinterziehung}

Marelli (1984) untersucht eine monopolistische Firma, die eine Umsatzsteuer mit dem Satz $t$ auf ihren Umsatz $R(Q)$ abführen muß. Der Umsatz ist eine Funktion der abgesetzten Menge $Q$ des von dem Monopolisten hergestellten Verbrauchsgutes. Die Kosten sind eine Funktion $C(Q)$. In diesem Lehrbuch-Modell wird nun die Steuerhinterziehung berücksichtigt. Der Fiskus kennt den Umsatz nur, wenn er erfolgreich eine Betriebsprüfung durchführt. Zielfunktion des Monopolisten sei der Erwartungsnutzen des Gewinns. Die Firma sei risikoavers. Ihre Maximierungsaufgabe ist

$$
\begin{array}{ll}
\max _{Q, H} & q U((1-t) R(Q)-C(Q)+t H) \\
& +p U((1-t) R(Q)-C(Q)-\alpha t H) \\
\text { u.d.N. } & 0 \leqq H \leqq R(Q)
\end{array}
$$

$H$ bezeichnet hier den hinterzogenen Umsatz.

Notwendige Bedingungen für eine innere Lösung sind

$$
\begin{gathered}
q U^{\prime}(G)-p U^{\prime}(P) \alpha=0 \\
\left(q U^{\prime}(G)+p U^{\prime}(P)\right)\left((1-t) R^{\prime}-C^{\prime}\right)=0
\end{gathered}
$$

Aus (2.18) folgt $(1-t) R^{\prime}=C^{\prime}$ : Die abzusetzende Menge $Q^{*}$ wird unabhängig von der Hinterziehung bestimmt. Die Bedingung Grenzerlös nach Steuer gleich Grenzkosten charakterisiert auch das Gewinnmaximum eines Monopolisten, der keine Steuern hinterzieht. Ein steuerunehrlicher Monopolist überwälzt die Umsatzsteuer genauso wie ein steuerehrlicher Alleinanbieter auf die Nachfrager (Marelli, 1984, S. 197): $Q^{*}$ ist unabhängig von $H^{*}$. Der hinterzogene Umsatz $H^{*}$ ist dagegen abhängig von $Q^{*}$. Die Größe $(1-t) R\left(Q^{*}\right)-C\left(Q^{*}\right)$ spielt in dem Modell die Rolle eines vorgegebenen Einkommens für die Hinterziehung. Die komparativ-statischen 
Ergebnisse entsprechen daher im wesentlichen den Zusammenhängen, die bereits aus dem Grundmodell bekannt sind (Marelli, 1984, S. 198 - 199).

Für verschiedene Modifikationen des Modells sei auf Marelli (1984) verwiesen. Mir kam es darauf an zu zeigen, wie das Lehrbuchmodell eines umsatzsteuerpflichtigen Monopolisten mit der Steuerhinterziehung verknüpft werden kann. Vor Schlußfolgerungen für die Praxis der Umsatzbesteuerung sei ausdrücklich gewarnt. Für die Analyse des Steuerbetrugs in dem System der Allphasenumsatzsteuer mit Vorsteuerabzug reicht das Modell nicht aus. In diesem System muß nicht nur für Verkäufe an Konsumenten, sondern auch für Verkäufe an andere Unternehmen Umsatzsteuer berechnet und abgeführt werden. Das belieferte Unternehmen kann diese sogenannte Vorsteuer bei Vorlage der Rechnung von den Umsatzsteuern, die es selbst abführen muß, abziehen. Wenn alle Vorsteuern geltend gemacht werden und die Finanzämter prüfen, ob die entsprechenden Umsatzsteuern eingegangen sind, bestehen keine Chancen, diese zu hinterziehen ${ }^{10}$. Trotzdem gibt es die Umsatzsteuerhịnterziehung. Für viele Waren werden keine Umsatzsteuern abgeführt, obwohl sie bis zu ihrer Fertigstellung verschiedene Unternehmen durchlaufen. Man kann sie auf jeder Stufe nur ohne Rechnung erwerben. Die Hinterziehung der Allphasenumsatzsteuer setzt daher eine gewisse Kooperation der beteiligten Unternehmen voraus. Eine formale Analyse dieser Zusammenarbeit gibt es bisher nicht.

\subsubsection{Hinterziehung von Arbeitseinkommen}

Pencavel (1979) läßt den Stpfl. arbeiten, bevor er veranlagt wird und schließlich sein Einkommen nach Steuer und Strafe konsumieren kann. Pro Arbeitsstunde $h$ verdient er einen Lohn $w$. Das Einkommen $w h$ unterliegt dem Tarif $t(w h)^{\sigma}$. Entscheidungsvariable des Stpfl. sind das Arbeitsangebot $h$ und das deklarierte Einkommen $D$. Die Budgetbeschränkungen sind

$$
\begin{aligned}
& G=w h-t D^{\sigma} \\
& P=w h-t(w h)^{\sigma}-\alpha t\left((w h)^{\sigma}-D^{\sigma}\right)
\end{aligned}
$$

Im Vergleich zu den entsprechenden Beschränkungen des Grundmodells ist das exogene Einkommen durch wh ersetzt worden.

Die Nutzenfunktion des Stpfl. enthält mit endogenem Arbeitseinkommen neben dem Konsum die Arbeitszeit $h$ als zweites Argument. Pencavel verwendet speziell die additiv separable Funktion $V(C)+W(h)$ mit den Eigenschaften $V^{\prime}>0, W^{\prime}<0, V^{\prime \prime}, W^{\prime \prime}<0$ und zusätzlich einer abnehmenden absoluten Risikoaversion $-V^{\prime \prime}(C) / V^{\prime}(C)$.

\footnotetext{
${ }^{10}$ Zusätzlich können die Behörden bei jedem einzelnen Unternehmen auf die Relation von deklariertem Umsatz und deklarierten Einkäufen achten.
} 
Die Anzahl der Argumente der Nutzenfunktion unterscheidet die Modelle von Haushalten und Unternehmen bei endogener Steuerbasis. Einziges Argument der Zielfunktion eines Unternehmens sind in der ökonomischen Theorie die finanziellen Überschüsse. Dem Unternehmen ist die produzierte Gütermenge, die Zahl der Beschäftigten etc. als solche gleichgültig. Die Wohlfahrt eines Haushalts wird jedoch auch direkt von seiner Arbeitsleistung beeinflußt, und nicht nur durch den Konsum, den sie ermöglicht. Die unterschiedlichen Zielfunktionen der Unternehmen und Haushalte haben Konsequenzen. Die Produktionsentscheidungen und andere Entscheidungen der Unternehmen können eventuell unabhängig von der Steuerhinterziehung getroffen werden (siehe den vorangehenden Abschnitt und Marelli/Martina, 1988, S. 57 f.). Haushalte müssen dagegen simultan über ihr Arbeitsangebot und das deklarierte Einkommen entscheiden. Die Konsequenz dieser Struktur zeigen die Ergebnisse von Pencavel.

Der Haushalt maximiert $E(V(C)+W(h))=q V(G)+p V(P)+W(h)$ durch die Wahl

$$
h^{*}=h^{*}(p, \alpha, t, \sigma, w)
$$

und

$$
D^{*}=D^{*}(p, \alpha, t, \sigma, w)
$$

Das deklarierte Einkommen $D^{*}$ ist eine "unilluminating function" (Cowell, 1986, S. 196) der exogenen Variablen: Die Wirkung jedes der fünf Parameter auf $D^{*}$ ist unbestimmt. "... the predominance of unambiguous sign implications" im Fall eines exogenen Einkommens verkehrt sich bei endogenem Arbeitseinkommen "into a predominance of ambiguities" (Pencavel, 1979, S. 121). Betrachten wir z.B. eine Erhöhung der Entdeckungswahrscheinlichkeit. Bei gegebenem Einkommen führt sie zu einem höheren deklarierten Einkommen und einer höheren Steuerbelastung. Der Stpfl. reduziert nun möglicherweise seine Arbeitszeit $h^{*}$. Das fallende Einkommen $w h^{*}$ aber führt zu einem geringeren deklarierten Einkommen. Der Gesamteffekt ist nicht vorhersagbar (ebenda, S. 121).

Pencavel ließ sich nicht beeindrucken und berechnete die Vorzeichen des hinterzogenen Einkommens $H^{*}=w h^{*}-D^{*}$, um zu eindeutigen Aussagen zu gelangen. Mit einem endogenen Einkommen gilt nur zufällig $d H^{*}=-d D^{*}$, so daß aus einem unbestimmten Vorzeichen von $d D^{*}$ nicht auch eine unbestimmte Reaktion des hinterzogenen Einkommens folgt. Mit proportionalem Tarif $(\sigma=1)$ erhält Pencavel folgende Resultate: $H^{*}$ sinkt mit $p$ und $\alpha$. Die Wirkung von $t$ und $w$ ist unbestimmt. Mit progressivem Tarif $(\sigma>1)$ ist die Wirkung aller fünf Parameter auf das hinterzogene Einkommen unbestimmt. Zumindest für den proportionalen Tarif sind höhere Kontrollen und Strafen erfolgreiche Mittel gegen die Steuerhinterziehung. Bei progressivem Tarif kann man sich auch dessen nicht sicher sein.

In "offiziellen" Beschäftigungsverhältnissen werden die Arbeitseinkommen in 
den meisten Ländern so hoch mit einer Lohnsteuer belastet, daß eine abschließende Einkommensteuerveranlagung zu keiner zusätzlichen Zahlungsverpflichtung der Arbeitnehmer führt. Das Modell von Pencavel scheint daher für unselbständig Beschäftigte nicht relevant zu sein. Mit Sandmo (1981), Wiegard (1984) und Cowell (1985) kann man ihm jedoch eine andere Interpretation geben, wenn man von folgender Sichtweise ausgeht: Die Wirtschaft ist in einen offiziellen und einen inoffiziellen Sektor gespalten. Die Unternehmen in dem ersten Sektor ziehen den Beschäftigten Lohnsteuern ab, so daß deren Steuerschuld abgegolten ist. In dem Sektor, der im "Schatten" liegt, werden alle Abgaben hinterzogen. Wenn ein einzelner Haushalt in beiden Sektoren beschäftigt sein kann, $w_{l}\left(w_{i}\right)$ der Lohnsatz in dem offiziellen Sektor (der Schattenwirtschaft) und $h_{l}\left(h_{i}\right)$ seine Arbeitszeit in diesem Sektor ist, lauten die beiden Budgetbeschränkungen des Haushalts

$$
\begin{aligned}
G & =w_{l} h_{l}(1-t)+w_{i} h_{i} \\
& =\left(w_{l} h_{l}+w_{i} h_{i}\right)(1-t)+t w_{i} h_{i} \\
P & =\left(w_{l} h_{l}+w_{i} h_{i}\right)(1-t)-\alpha t w_{i} h_{i}
\end{aligned}
$$

Ist neben dem Nettoeinkommen die Summe $h=h_{l}+h_{i}$ das zweite Argument seiner Nutzenfunktion, entsprechen sich dieses Modell und das Modell von Pencavel ${ }^{11}$ - und damit auch ihre Ergebnisse ${ }^{12}$. Cowell (1985) ersetzt daher die additive Separabilität der Nutzenfunktion durch eine funktionale Separabilität: Funktionen der Form

$$
U(C, h)=f_{1}\left(f_{2}(h)+f_{3}(h) C\right)
$$

mit $f_{1}, f_{2}$ und $f_{3}$ als zunehmende Funktionen führen dazu, daß die Entscheidungen über die Gesamtarbeitszeit und die Arbeit in der Schattenwirtschaft in folgendem Sinne separabel sind. $h$ ist allein von dem sicheren Nettolohnsatz in dem offiziellen Sektor abhängig: $h^{*}=h^{*}\left(w_{l}(1-t)\right)$. Das inoffizielle Arbeitsangebot ist von $h^{*}$ und vier Parametern abhängig, die den Ertrag der Schwarzarbeit bestimmen: $h_{i}^{*}=h_{i}^{*}\left(h^{*}, w_{i}, t, p, \alpha\right)$. Die offizielle Arbeitszeit ergibt sich aus der Differenz von $h^{*}$ und $h_{\boldsymbol{i}}^{*}$. Das Entscheidungsproblem des Haushalts kann also in zwei Stufen gespalten werden. Zuerst wird über die Gesamtarbeitszeit und anschließend über die "unsichere Anlage" eines Teils dieser Zeit entschieden (vgl. Drèze/Modigliani, 1972, S. 321 f.). Es liegt auf der Hand, daß nun mehr über die Wirkung finanzpolitischer Parameteränderungen auf das Verhalten des Steuerhinterziehers ausgesagt werden kann, wenn man neben der funktionalen Separabilität die gewohnten Annahmen über den Verlauf der Arrow-Pratt-Maße trifft. Die Zusammenhänge

\footnotetext{
${ }^{11} \mathrm{DaB}$ legale Arbeit und die Arbeit in der Schattenwirtschaft sich z.B. durch die Dauer und Sicherheit der Beschäftigungsverhältnisse unterscheiden, wird hier nicht berücksichtigt.

${ }^{12}$ Siehe jedoch Wiegard (1984), der zum Teil bestimmtere Resultate als Pencavel erzielt, obwohl beide dieselbe additiv separable Nutzenfunktion verwenden.
} 
entsprechen durchweg denjenigen, die man aufgrund des Grundmodells erwartet hätte (vgl. Cowell, 1985, S. 29).

Unterschiede ergeben sich, wenn $h_{l}$ fest vorgegeben ist: Wer sich einmal für einen bestimmten Beruf entschieden hat, kann seine Arbeitszeit in seinem offiziellen Beschäftigungsverhältnis kaum variieren, über das Ausmaß zusätzlicher Schwarzarbeit aber schon frei entscheiden. In diesem realistischen Fall besteht z.B. zwischen $p$ bzw. $\alpha$ und der in der Schattenökonomie verbrachten Arbeitszeit auch ohne die Annahme einer funktional separablen Nutzenfunktion ein negativer Zusammenhang, wenn das Arbeitsangebot mit $w_{i}$ steigt (Cowell, 1985, S. 29).

Zu den zwei Formen der Separabilität, die in der Literatur Verwendung finden, soll eine kurze Bemerkung festgehalten werden. Unterschiedliche Nutzenfunktionen führen im allgemeinen zu unterschiedlichen Ergebnissen. Auf dem Boden der angewandten Theorie kann jedoch nicht ohne weiteres entschieden werden, welche Präferenzordnungen der Entscheidungsträger "defensible" und "reasonable" sind (Cowell, 1985, S. 24 und 32). Daß bestimmte Funktionen eher als andere Ergebnisse generieren, die der "armchair evidence" ${ }^{13}$ entsprechen, ist kein Grund dafür, sie mit jenen Prädikaten zu versehen.

Was leistet die Literatur zur Hinterziehung von Arbeitseinkommen zum Verständnis der Besteuerungspraxis? Für Selbständige und selbständige Schwarzarbeiter sind die Modelle instruktiv. Viele Haushalte können jedoch nicht einmal dann als Selbständige bezeichnet werden, wenn sie im "Schatten" arbeiten. Auch für die Schwarzarbeit werden zumeist eine gewisse Kapitalausstattung und geschäftliche Verbindungen benötigt, die im Besitz von (im Handelsregister eingetragenen) Firmen sind. Die Schwarzarbeit zeichnet sich daher in vielen Fällen durch eine Kooperation von "offiziellen" Firmen und mit ihrer Hilfe beschäftigten Schwarzarbeitern aus (vgl. Wenig, 1988, S. 4), wenn man nicht sogar von unselbständig beschäftigten Schwarzarbeitern sprechen muß. An der Hinterziehung der Abgaben sind damit zwei Parteien beteiligt, so daß die Modellierung dieses Vorgangs als einsame Entscheidung eines Haushalts nicht sehr überzeugend wirkt.

\subsection{Interdependenzen zwischen Steuerhinterziehern}

Eine wichtige Einsicht der Volkswirtschaftslehre ist, daß für die Gesamtheit der Mitglieder einer Wirtschaft nicht gelten muß, was für einzelne bei unverändertem Verhalten aller anderen gilt. Dieser Einsicht folgend ist in vielen Bereichen der Wirtschaftstheorie das Zusammenspiel vieler einzelner Wirtschaftseinheiten

\footnotetext{
${ }^{13}$ Der Ausdruck ist Cowell (1986, S. 193) entnommen.
} 
gründlich untersucht worden. Von der Literatur zur Steuerhinterziehung kann man das nicht behaupten. Sie erzeugt den Eindruck, als ob die Steuerhinterziehung nur einen einzigen Steuerpflichtigen und vielleicht noch die Finanzbehörde betrifft. Da es jedoch nicht einen, sondern viele Steuerhinterzieher geben wird, ist zu erwarten, daß es Zusammenhänge zwischen den Entscheidungsproblemen verschiedener Steuerpflichtiger gibt.

Die Überlegungen, die über ein Partialmodell hinausgehen und Interdependenzen zwischen Steuerhinterziehern thematisieren, sollen im folgenden dargestellt werden. Watson (1984) analysiert die Steuerhinterziehung im Arbeitsmarktgleichgewicht. Cowell/Gordon (1988), Falkinger (1988) und Gottlieb (1985) berücksichtigen, daß ein verbreiteter Steuerbetrug die öffentlichen Ausgaben kürzt und das Ausmaß der Bereitstellung öffentlicher Güter die Steuerehrlichkeit beeinflussen kann. Schlicht (1985), Benjamini/Maital (1985) und Gordon (1989) behandeln den Fall, daß die Neigung eines Steuerpflichtigen zur Hinterziehung von der Steuermoral anderer Bürger abhängt. Schließlich soll gezeigt werden, daß es für Steuerhinterzieher einen Anreiz gibt zusammenzuarbeiten, statt isoliert gegen den Fiskus zu Felde zu ziehen. Auf das Modell von Marelli/Martina (1988, S. 66 - 68), in dem die Entdeckungswahrscheinlichkeit eines gewinnsteuerpflichtigen Dyopolisten positiv von der Differenz zwischen dem durchschnittlichen deklarierten Gewinn der Branche und dem von ihr angegebenen Gewinn abhängt, soll hier nur verwiesen werden, da sie zu sehr differenzierten Ergebnissen kommen. Die Interdependenzen zwischen den Steuerhinterziehern kommen also einmal über Marktpreise, das öffentliche Budget, durch verbundene Präferenzen, durch Kooperation und durch endogene Entdeckungswahrscheinlichkeiten zustande.

\subsubsection{Steuerhinterziehung und}

\section{Arbeitsmarktgleichgewicht}

In Watson (1984) ist die Wirtschaft in einen Sektor l, in dem die Löhne der abhängig Beschäftigten von der Lohnsteuer erfaßt werden, und einen Sektor $s$ von Selbständigen bzw. selbstbeschäftigten Arbeitern gespalten. $n$ Haushalte bieten ihre Arbeitskraft unelastisch auf genau einem der zwei entsprechenden Arbeitsmärkte an. Der "Lohn" der Selbstbeschäftigten $w_{s}$ kann hinterzogen werden. Der Lohn $w_{l}$ der unselbständigen kann nicht hinterzogen werden. Alle Arbeiter sind bis auf eine Ausnahme identisch: Sie unterscheiden sich durch ihre Entdeckungswahrscheinlichkeit im Sektor $s$. Watson begründet die Annahme damit, daß "the ability to avoid detection varies across workers" (Watson, 1984, S. 233). Abbildung 2.5 gibt die Vorstellung von Watson über die Entdeckungswahrscheinlichkeiten $p_{i}$ der Arbeiter $i=1, \cdots, n$ wieder. Jeder von ihnen löst für parametrisch gegebene Lohnsätze folgendes Problem. Er überlegt zunächst, wieviel von $w_{s}$ er 
Abbildung 2.5: Unterschiedliche Gelegenheiten zur Steuerhinterziehung

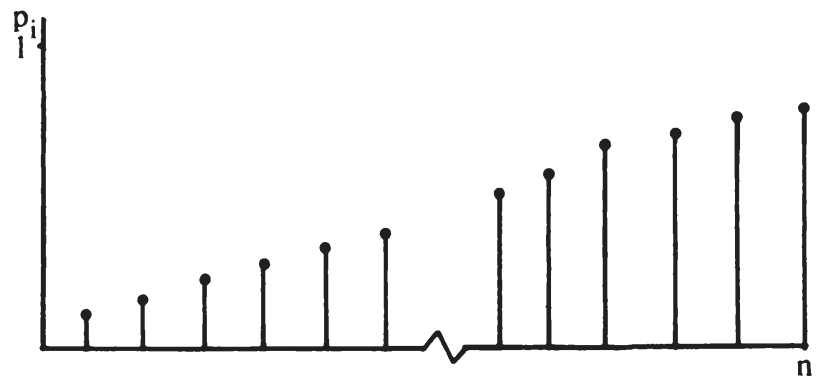

hinterzieht, gesetzt er arbeitet selbständig. Den Erwartungsnutzen in Markt $s$ vergleicht er dann mit dem Nutzen des sicheren Einkommens $w_{l}(1-t)$, das er auf Markt $l$ erzielen könnte. Resultiert eine positive Differenz, arbeitet er auf Markt s. Ist sie negativ, tritt er in eine offizielles Beschäftigungsverhältnis ein.

Die (identischen) Arbeitsnachfragefunktionen in den zwei Sektoren hängen negativ von $w_{0}$ bzw. $w_{l}$ ab. Diese beiden Funktionen determinieren zusammen mit den Entscheidungen aller Arbeiter die Gleichgewichtslöhne $w_{s}^{G}$ und $w_{l}^{G}$ und die Aufteilung der Arbeiter zwischen den Märkten. Der Lohn $w_{s}^{G}$ ist kleiner als $w_{l}^{G}$ : "... the gains that might be made by those who are more able to avoid detection are partially eliminated by wage declines in markets in which evasion is possible" (Watson, 1984, S. 243). Wäre dagegen in keinem Markt eine Hinterziehung möglich, würde man $w_{s}^{G}=w_{l}^{G}$ erwarten.

Ausgehend von einem Gleichgewicht untersucht Watson, wie die Anzahl $n_{s}$ der in Sektor $s$ beschäftigten Arbeiter und der Anteil des deklarierten Lohnes am tatsächlichen Einkommen dieser Arbeiter von den Parametern des Modells abhängen. Ich gebe nur sein Ergebnis bezüglich einer proportionalen Erhöhung der Entdeckungswahrscheinlichkeit aller Haushalte an. Verschärfte Kontrollen lassen einen Teil der Arbeiter von Markt $s$ nach Markt $l$ wechseln, da sich für sie der Steuerbetrug nicht mehr lohnt. Die Arbeiter, die in Markt $s$ verbleiben, deklarieren ceteris paribus einen höheren Anteil ihres Lohnes. Neben dem direkten Effekt einer höheren Entdeckungswahrscheinlichkeit bei gegebenen Lohnsätzen tritt ein indirekter Effekt, da sich aufgrund der Wanderung der Arbeiter zwischen den beiden Märkten die Löhne ändern. $w_{0}^{G}$ steigt mit der sinkenden Zahl der Anbieter auf Markt $s$. Mit $w_{s}^{G}$ steigt der Anteil des deklarierten Einkommens weiter an (bzw. der Anteil des hinterzogenen Einkommens sinkt weiter), wenn man mit Watson eine zunehmende relative Risikoaversion annimmt. Die Reaktion des hinterzogenen Einkommens eines Arbeiters, der auf Markt $s$ bleibt, ist jedoch unbestimmt. Über 
das erwartete Steueraufkommen von allen Arbeitern macht Watson leider keine Aussagen.

Die Arbeit von Watson ist ein erster Schritt zu einer Analyse der Steuerhinterziehung im Marktgleichgewicht. Man muß aus ihr den Schluß ziehen, daß infolge der Preisbewegungen, die finanzpolitische Maßnahmen über ein verändertes Verhalten vieler einzelner Stpfl. auslösen, den Aussagen der Partialmodelle gegenüber Vorsicht angebracht ist.

\subsubsection{Steuerhinterziehung und die Bereitstellung öffentlicher Güter}

Das Steueraufkommen verwendet der Staat für die Bereitstellung öffentlicher Leistungen. Wenn ein einziger Stpfl. hinterzieht, wird der entsprechende Aufkommensverlust die öffentlichen Leistungen kaum beeinflussen. Wenn Millionen von Bürgern ihre Steuerschuld verkürzen, müssen die öffentlichen Leistungen jedoch gekürzt werden. Dies berührt die Wohlfahrt jedes einzelnen Stpfl. und kann ihn dazu veranlassen, seine Deklarationsentscheidung zu überdenken. Gottlieb (1985), Falkinger (1988) und Cowell/Gordon (1988) berücksichtigen diesen Zusammenhang. Ich konzentriere mich hier auf den Aufsatz von Cowell/Gordon, da sie einer Frage nachgehen, die uns schon in den Partialmodellen beschäftigt hat: Welchen Einfluß hat die Steuerbelastung (der Steuersatz) auf die Hinterziehung? Cowell/Gordon fragen speziell: Kann durch die Berücksichtigung öffentlicher Güter der in empirischen Arbeiten gefundene positive Zusammenhang zwischen diesen Größen (siehe Clotfelter, 1983, dessen Ergebnis von Slemrod, 1985, jedoch nicht bestätigt wird) erklärt werden?

Betrachtet wird eine Wirtschaft mit $n$ identischen Individuen. Der Erwartungsnutzen jedes Stpfl. ist

$$
E(U(C, Z))=q U(G, Z)+p U(P, Z)
$$

wobei $G$ und $P$ durch (1.1) und (1.2) definiert sind. Das zusätzliche Argument der Nutzenfunktion ist das durch Einkommensteuern finanzierte öffentliche Gut. Hinterzieht ein bestimmter Stpfl. $S=t H$ und alle übrigen Stpfl. jeweils $\bar{S}=t \bar{H}$, ist das Aufkommen pro Kopf gleich ${ }^{14}$

$$
A=t Y-\frac{n-1}{n}(q-p \alpha) \bar{S}-\frac{1}{n}(q-p \alpha) S
$$

Gibt es sehr viele Stpfl. $(n \rightarrow \infty)$, gilt

$$
A=t Y-(q-p \alpha) \bar{S}
$$

\footnotetext{
${ }^{14}$ Die Kosten der Steuerprüfungen, die Cowell/Gordon berücksichtigen, werden hier vernachlässigt, da sie für das Ergebnis der Analyse keine Rolle spielen.
} 
Dieses Aufkommen wird in $(1 / \psi) A$ Einheiten des öffentlichen Gutes $Z$ umgesetzt, das jedem Stpfl. zur Verfügung steht.

Wie bestimmt sich die hinterzogene Steuer eines einzelnen Stpfl.? Der einzelne geht bei seiner Entscheidung $S^{*}$ davon aus, daß sie einen zu vernachlässigenden Einfluß auf das Ausmaß der Bereitstellung des öffentlichen Gutes hat. Zweitens soll er davon ausgehen, daß die Entscheidungen aller anderen Individuen von seiner Deklarationsentscheidung unberührt bleiben. Unter diesen Voraussetzungen sind

$$
q U_{C}(G, Z)-p U_{C}(P, Z) \alpha=0
$$

und

$$
\Delta=q U_{C C}(G, Z)+p U_{C C}(P, Z) \alpha^{2}=0
$$

die Bedingungen erster und zweiter Ordnung für ein Maximum von $E(U(C, Z))$. Dabei ist $U_{C}$ die erste Ableitung nach $C$, etc.

(2.19) bestimmt $S^{*}$ als Funktion von (u.a.) Z. Ein Cournot-Nash-Gleichgewicht ist erreicht, wennn $S^{*}=\bar{S}$ gilt: Die Entscheidungen aller (identischen) Stpfl. sind kompatibel; für keinen Stpfl. lohnt es sich, von der Entscheidung $S^{*}$ abzugehen. $S^{*}=\bar{S}=S^{G}$ und $Z$ werden von den zwei Gleichungen (2.19) und (2.20) bestimmt.

$$
Z=\frac{1}{\psi}\left(t Y-(q-p \alpha) S^{G}\right)
$$

Differenziert man diese Gleichungen nach $t$, erhält man die Wirkung eines höheren Steuersatzes auf $S^{G}$. Aus (2.19) ergibt sich:

$$
\begin{aligned}
\frac{\partial S^{G}}{\partial t}= & \Delta^{-1}\left[\left(q U_{C C}(G, Z)-p U_{C C}(P, Z) \alpha\right) Y\right. \\
& \left.-\left(q U_{C Z}(G, Z)-p U_{C Z}(P, Z) \alpha\right) \frac{\partial Z}{\partial t}\right]
\end{aligned}
$$

Die Änderung der hinterzogenen Steuer eines einzelnen Stpfl. ist zu klein, um auf $Z$ Einfluß zu haben. Unendlich viele kleine Änderungen von $S^{G}$ summieren sich aber zu einer endlichen Änderung von $Z$, so daß neben dem üblichen direkten Effekt von $t$ zu beachten ist, daß sich durch die Steuererhöhung die Versorgung mit dem öffentlichen Gut ändert (siehe Cowell/Gordon, 1988, S. 307 - 309, für eine rigorose Ableitung dieses Zusammenhangs). Setzt man

$$
\frac{\partial Z}{\partial t}=\frac{1}{\psi}\left(Y-(q-p \alpha) \frac{\partial S^{G}}{\partial t}\right)
$$

in (2.21) ein, ergibt sich (Cowell/Gordon, 1988, S. 309):

$$
\begin{aligned}
\frac{\partial S^{G}}{\partial t}= & \left(\Delta^{G}\right)^{-1}\left\{\left[q U_{C C}(G, Z)-p U_{C C}(P, Z) \alpha\right] Y\right. \\
& \left.-\left[q U_{C Z}(G, Z)-p U_{C Z}(P, Z) \alpha\right] Y / \psi\right\}
\end{aligned}
$$


wobei $\Delta^{G}=\Delta-\left[q U_{C Z}(G, Z)-p U_{C Z}(P, Z) \alpha\right](q-p \alpha) / \psi$ ist. Der Nenner dieses Ausdrucks ist negativ, wenn das Cournot-Nash-Gleichgewicht stabil ist (Cowell/Gordon, 1988, S. 310). Der Zähler enthält zwei Terme. Der erste hat mit einer abnehmenden absoluten Risikoaversion ein positives Vorzeichen und spricht (wegen des negativen Nenners) für einen negativen Zusammenhang von Steuerbelastung und -hinterziehung. Dahinter steckt der negative Einkommenseffekt, auf den Yitzhaki (1974) hingewiesen hat. Der rechte Term im Zähler (exclusive des Minuszeichens) hat das Vorzeichen von $\partial S^{*} / \partial Z$. Es handelt sich hier um einen zweiten "Einkommens"-Effekt, der auf die Änderung der Versorgung mit dem öffentlichen Gut zurückgeht, die aus einer höheren Besteuerung sehr vieler Individuen resultiert (Cowell/Gordon, 1988, S. 310).

Um den Nettoeffekt zu bestimmen, muß von einer speziellen Nutzenfunktion ausgegangen werden. Es soll hier nur der Fall $U_{Z} / U_{C}=m(Z)$ betrachtet werden: Die Grenzrate der Substitution zwischen $C$ und $Z$ ist unabhängig von $C$, d.h. auch unabhängig davon, ob der Stpfl. entdeckt wird oder nicht. Aus

$$
\frac{\partial m(Z)}{\partial C}=\frac{U_{Z C} U_{C}-U_{Z} U_{C C}}{\left(U_{C}\right)^{2}}=0
$$

folgt $U_{Z C}=m U_{C C}$. Setzt man diese Beziehung in (2.22) ein, gelangt man zu

$$
\frac{\partial S^{G}}{\partial t}=-\left(\Delta^{G}\right)^{-1}\left[\left(q U_{C C}(G, Z)-p U_{C C}(P, Z) \alpha\right] Y(m / \psi-1)\right.
$$

Das Vorzeichen dieses Ausdrucks hängt mit abnehmender absoluter Risikoaversion von $m / \psi-1$ ab. Diese Bedingung gibt an, ob die Individuen mit dem öffentlichen Gut unter- oder überversorgt sind. Der Versuch, den Anteil des Staates an dem Einkommen der Wirtschaft durch eine Steuererhöhung zu steigern, hat auf den Erwartungsnutzen jedes Stpfl. im Gleichgewicht nämlich folgenden Einfluß:

$$
\begin{aligned}
\frac{\partial E(U(C, Z))}{\partial t} & =Y\left[-q U_{C}(G, Z)-p U_{C}(P, Z)+\left(q U_{Z}(G, Z)+p U_{Z}(P, Z)\right) / \psi\right] \\
& =Y\left[q U_{C}(G, Z)+p U_{C}(P, Z)\right](m / \psi-1)
\end{aligned}
$$

Dabei sind das Enveloppen-Theorem und die Beziehung $U_{Z}=m U_{C}$ ausgenutzt worden. Ist die Änderung des Erwartungsnutzens z.B. positiv, "then public goods are under-provided since the individual would welcome an expansion of the public sector with its consequent implications of enforced taxation" (Cowell/Gordon, 1988, S. 312).

Vergleicht man nun die Änderungen des Nutzens und der hinterzogenen Steuer $S^{G}$, ergibt sich: Zwischen Steuerbelastung und Hinterziehung besteht ein positiver (negativer) Zusammenhang, wenn eine Unterversorgung (Überversorgung) mit dem öffentlichen Gut vorliegt. Die ökonomische Logik für dieses überraschende 
Ergebnis ist: "If public goods are under-provided (as definded), then when the government increases their provision (via a higher tax rate), it runs the risk of making individuals on balance feel better off. Under decreasing absolute risk aversion this will make them increase the size of their gamble, i.e. evade more tax (Cowell/Gordon, 1988, S. 313).

Dieses Verhalten ist nun nicht nur überraschend, sondern geradewegs unerfreulich, wenn eine Unterversorgung mit öffentlichen Gütern besteht. Versucht der Staat nämlich, dieser Situation durch eine Erhöhung von $t$ abzuhelfen, ist nicht sicher, daß das Steueraufkommen steigt. Der Versuch, die Grenzrate der Transformation pro Kopf ${ }^{15} \psi$ und die Grenzrate der Substitution $m$ zwischen dem privaten und dem öffentlichen Gut anzugleichen, kann fehlschlagen, da die Individuen sich in einem "prisoner's dilemma" befinden und nach individuell-rationalen Gesichtspunkten entscheiden.

Wenn Steuerzahler (aus dem dargestellten Modell) argumentieren, daß sie hinterziehen, weil Steuern für überflüssige öffentliche Ausgaben verwendet werden, regt sich der Verdacht, daß es sich bei dem Argument um eine "rationalization of otherwise determined evading-decisions" handelt (Falkinger, 1988, S. 388): Individuell- rationale Entscheidungen werden mit dem Anschein kollektiver Rationalität versehen. Ist dieser Verdacht richtig, hätten Cowell/Gordon (1988, S. 320) keinen Grund, sich über das von ihnen erzielte Ergebnis so zerknirscht zu äußern und die mangelnde Verläßlichkeit ökonomischer Intuition zu beklagen: “... resting an explanation for a positive tax rate effect on public goods being under-provided is unsatisfactory, since intuition seems to suggest the opposite, i.e. that individuals are more likely to increase evasion after a tax rise when they feel that government services do not represent good value for their tax contributions".

\subsubsection{Steuerhinterziehung als Epidemie}

Ein Stpfl. wird eventuell mehr hinterziehen, wenn er weiß, daß auch viele andere nicht steuerehrlich sind. Der Steuerbetrug kann ansteckend wirken. Empirische Studien deuten darauf hin, daß "the more tax evaders a taxpayer knows, the more likely he is to evade taxes himself" (Spicer/Lundstedt, 1976, S. 297). Schlicht $(1985)^{16}$, Benjamini/Maital (1985) und Gordon (1989) untersuchen die Konsequenzen dieser Annahme über die Präferenzen der Stpfl. Die Interdependenz zwischen

\footnotetext{
${ }^{15} \psi$ gibt an, um welchen Betrag der (erwartete Konsum) jedes Stpfl. sinkt, wenn eine zusätzliche Einheit des öffentlichen Gutes finanziert werden soll. $m=\psi$ ist die Samuelson-Bedingung für eine pareto-optimale Bereitstellung des öffentlichen Gutes, auch wenn sie hier etwas ungewöhnlich aussieht.

${ }^{16}$ Schlichts Modell ist nicht speziell für die Steuerhinterziehung, sondern für Gesetzesverstöße im allgemeinen formuliert.
} 
den Stpfl., die durch die Ansteckung entsteht, wird bei Gordon besonders klar, so daß ich mich im folgenden primär auf diesen Aufsatz beziehe.

In Gordon (1989) hat jeder Haushalt in Periode $t$ die Erwartungsnutzenfunktion ${ }^{17}$

$$
q U(G)+p U(P)-\left(v+r\left(1-e_{t-1}\right)\right) H_{t}
$$

Die Steuerhinterziehung verursacht neben der Geldstrafe zusätzliche Kosten. $v$ sind die "privaten psychischen Kosten" pro DM nicht deklarierten Einkommens: "a false income declaration may induce anxiety, guilt or a reduction in self-image" (Gordon, 1989, S. 798). $v$ soll von Individuum zu Individuum unterschiedlich sein. $F\{v\}, 0 \leqq v \leqq v^{+}$, ist die Verteilungsfunktion des Merkmals $v$ in der Bevölkerung. Sie gibt an, wieviel Prozent aller Stpfl. höchstens Kosten in Höhe von $v$ haben. $r\left(1-e_{t-1}\right)$ sind die für alle gleichen "sozialen psychischen Kosten" pro DM nicht deklarierten Einkommens. Dabei ist $r$ ein Parameter und $e_{t-1}$ der Prozentsatz der Bevölkerung, der in Periode $t-1$ hinterzogen hat. Gordon nimmt an, daß die Stpfl. erfahren, wieviel Prozent der Bevölkerung in der Vergangenheit steuerehrlich war. Diesen Prozentsatz nehmen sie als Schätzwert für den Teil aller Stpfl., die die Steuerhinterziehung moralisch verurteilen (Gordon, 1989, S. 801). Je mehr die Hinterziehung verurteilt wird, desto größer sind die seelischen Kosten dieser Aktivität.

Ein Stpfl. mit dem Merkmal $v$ hinterzieht, wenn

$$
(q-p \alpha) \tau U^{\prime}(Y(1-\tau))-\left(v+r\left(1-e_{t-1}\right)\right)>0
$$

gilt. Sein Entscheidung ist damit abhängig von den Entscheidungen aller anderen Stpfl. in Periode $t-1$. Sind seine zusätzlichen Kosten der Hinterziehung nur groß genug, wird er trotz der Annahme $q-p \alpha>0$ nicht hinterziehen. Mit der Verteilungsfunktion $F\{v\}$ kann man nun den Prozentsatz der Stpfl. berechnen, die in Periode $t$ hinterziehen:

$$
e_{t}=F\left\{(q-p \alpha) \tau U^{\prime}(Y(1-\tau))-r\left(1-e_{t-1}\right)\right\}
$$

Gilt $e_{t} \neq e_{t-1}$, ist das System (noch) nicht im Gleichgewicht.

Die Lösung dieser Differenzengleichung - der gleichgewichtige Prozentsatz $e$ der Hinterzieher - hängt kritisch von der Form der Verteilung $F$ ab. Unabhängig von der Form von $F$ gilt jedoch: Wenn die psychischen Kosten der Hinterziehung (gemessen an den Parametern $v$ und $r$ ) im Vergleich zu dem Grenzertrag $(q-p \alpha) \tau U^{\prime}(Y(1-\tau))$ nicht zu groß (klein) sind, gibt es ein $e>0(e<1)$ (Gordon, 1989, S. 803). Möglicherweise existiert jedoch mehr als ein innerer Gleichgewichtswert des Anteils der Steuerzahler, die hinterziehen: Wegen der Ansteckung

\footnotetext{
${ }^{17}$ In diesem Abschnitt bezeichnet $t$ die Periode und $\tau$ den Steuersatz.
} 
deklarieren alle Stpfl. tendenziell gleich viel oder wenig. Zu diesem Ergebnis kommen auch Schlicht (1985) und Benjamini/Maital (1985). Gordon und Schlicht zeigen weiter, daß es instabile Gleichgewichte geben kann, die schwerwiegende Konsequenzen haben. Ein kleiner exogener Anreiz, etwas weniger Einkommen zu deklarieren, veranlaßt jeden einzelnen Stpfl. zunächst zu einer kleinen Änderung seines Verhaltens. Wenn der einzelne gewahr wird, daß alle anderen mehr hinterziehen, geht er selbst noch weiter. Die Ansteckung ruft einen kumulativen Verfall der Steuermoral hervor, selbst wenn der Anstoß von außen nur klein war:

Gordon untersucht neben der Existenz und Stabilität der Gleichgewichtslösung die Wirkung einer Erhöhung des Steuersatzes auf die Zahl der Hinterzieher und die Summe der nicht deklarierten Einkommen aller Individuen. Dabei geht er von einem inneren Gleichgewicht $e^{1}$ aus und nimmt an, daß das System stabil ist. Eine höhere Steuer läßt zunächst den Grenznutzen der ersten hinterzogenen DM steigen:

$$
\begin{gathered}
\frac{\partial\left[(q-p \alpha) \tau U^{\prime}((1-\tau) Y)-\left(v+r\left(1-e^{1}\right)\right]\right.}{\partial \tau}= \\
(q-p \alpha)\left[U^{\prime}((1-\tau) Y)-\tau U^{\prime \prime}((1-\tau) Y) Y\right]>0
\end{gathered}
$$

Damit steigt der Anteil der Hinterzieher in der Bevölkerung. In der nächsten Periode sind daher die psychischen Grenzkosten der Steuerhinterziehung gesunken, und die Zahl der ehrlichen Stpfl. nimmt weiter ab. In dem neuen Gleichgewicht ist der Anteil der Hinterzieher größer als in dem Ausgangsgleichgewicht $\left(e^{2}>e^{1}\right)$. Zusätzlich haben die Stpfl., die schon vor der Steuererhöhung nicht ehrlich waren, einen Anreiz, mehr als zuvor zu hinterziehen, da die psychischen Grenzkosten gesunken sind. In die andere Richtung weist wieder der mit einer abnehmenden absoluten Risikoaversion negative Einkommenseffekt der Besteuerung. Die Reaktion der Summe aller nicht deklarierten Einkommen kann daher zwar nicht eindeutig bestimmt werden. Ein positiver Zusammenhang zwischen der Besteuerung und diesem $\mathrm{MaB}$ des Abgabenwiderstands ist aber möglich.

Die Instabilität des Hinterziehungsverhaltens, die mit der Ansteckung verbunden sein kann, läßt Schlicht (1985, S. 266) vor der von der Marginalanalyse suggerierten Vorstellung warnen, "that slightly increasing incentives to unlawful behaviour will lead to a slightly lesser degree of law-obedience". Die Warnung ist sicher berechtigt. Es bleibt jedoch unklar, ob es viele Stpfl. geben wird, die sich anstecken lassen: Ist es mit anderen Worten rational, sich bei einer exogenen Entdeckungswahrscheinlichkeit zu Gesetzesverstößen anstecken zu lassen? Diese Frage heißt natürlich nicht mehr, als daß eine genauere Begründung der von Benjamini/Maital, Schlicht und Gordon postulierten Präferenzen erforderlich ist.

Auch ohne eine Ansteckung durch psychische Kosten der Hinterziehung könnte das Verhalten eines Stpfl. von anderen abhängig sein: Seine subjektive Schätzung der Entdeckungswahrscheinlichkeit könnte um so kleiner sein, je mehr erfolgreiche 
Steuerhinterzieher er kennt. Es müßte danach heißen, "the more successful tax evaders a taxpayer knows, the more likely he is to evade taxes himself".

\subsubsection{Versicherung der Folgen einer Steuerprüfung}

Die Literatur nimmt implizit an, daß ein Steuerhinterzieher sich gegen die Konsequenzen einer steuerlichen Prüfung nicht versichern kann. Tatsächlich ist auch keine Versicherungsgesellschaft bekannt, die gegen Prämie verspricht, im Fall einer entdeckten Steuerstraftat die nachzuzahlende Steuer und die Strafe zu tragen. Eine Versicherung muß jedoch nicht auf einem (öffentlichen) Markt zwischen Versicherungsnehmern, die einem Risiko ausgesetzt sind, und einem Versicherungsunternehmen, das bereit ist, viele unkorrelierte Risiken zu übernehmen, zustande kommen. Es können sich auch Individuen gegenseitig versichern, ohne daß die Existenz solcher Vereinbarungen öffentlich bekannt werden muß. Ich zeige im folgenden, wie sich zwei Steuerhinterzieher gegen die Folgen steuerlicher Prüfungen versichern könnten. Im Anschluß wird diskutiert, warum die beschriebene Kooperation eventuell nicht zustande kommt.

Das Beispiel baut auf dem Grundmodell auf. Nur geht es jetzt um zwei Stpfl., die d.E.h. indentische Präferenzen und ein gleich hohes Einkommen haben. Sie beschließen, sich gegen steuerliche Prüfungen zu versichern. Da sie unabhängig voneinander mit der Wahrscheinlichkeit $p$ geprüft werden, sind vier Fälle zu unterscheiden. Im ersten Fall werden beide nicht geprüft. Im zweiten Fall werden beide kontrolliert. In zwei weiteren Fällen wird jeweils nur einer geprüft. Die Wahrscheinlichkeiten der vier Zustände sind $q^{2}, p^{2}$ und zweimal $p q$. Für die ersten zwei Zustände vereinbaren die beiden Stpfl. keine Versicherung, da sie jeweils in derselben Lage sind. Interessant sind die zwei letzten Fälle. Die beiden Stpfl. vereinbaren, daß der, der nicht geprüft wird, dem anderen einen Betrag $X$ zahlt. Der Erwartungsnutzen jedes Stpfl. ist

$$
q^{2} U(G)+p^{2} U(P)+p q\left(U\left(G_{P}\right)+U\left(P_{G}\right)\right)
$$

$G$ ist das Nettoeinkommen des Stpfl., wenn beide nicht entdeckt werden. $P$ ist entsprechend definiert. $P_{G}$ ist der Konsum des Stpfl., wenn er selbst geprüft, sein Partner aber nicht kontrolliert wird. Entsprechend ist $G_{P}$ definiert.

$$
\begin{aligned}
G_{P} & =(1-t) Y+t H-X \\
P_{G} & =(1-t) Y-\alpha t H+X
\end{aligned}
$$

Wie wird $X$ bestimmt? Aus (2.23), der Konstanz der Summe $G_{P}+P_{G}$ bei gegebenem $H$ und der strikten Konkavität der Nutzenfunktion folgt, daß $X$ optimal 
festgelegt ist, wenn $G_{P}=P_{G}$ gilt. Mit (2.24) und (2.25) erhält man

$$
\begin{aligned}
(1-t) Y+t H-X^{*} & =(1-t) Y-\alpha t H+X^{*} \\
\Leftrightarrow \quad X^{*} & =\frac{(1+\alpha) t H}{2}
\end{aligned}
$$

Der Stpfl., der Glück gehabt hat, beteiligt sich zur Hälfte an der Steuernachzahlung und der Strafe des anderen. Der Erwartungsnutzen beider Partner ist mit der Versicherung $X^{*}$ größer als ohne Versicherung.

Es ist ein gegebenes hinterzogenes Einkommen unterstellt worden. Plausibel ist, daß infolge der Versicherung mehr hinterzogen wird. Quantitativ würde die Kooperation sich dann auf die Hinterziehung auswirken. An der qualitativen Abhängigkeit der Hinterziehung von den Parametern des Modells ändert sich jedoch nichts. Statt diese Punkte im Detail zu belegen, stelle ich die Frage, ob die Versicherung überhaupt zustande kommen kann.

Das Beispiel hat zwar gezeigt, daß ein Anreiz zu einer Versicherung des Steuerbetrugs besteht. Die "armchair evidence" deutet aber nicht auf die Verbreitung dieser Form der Kooperation hin. Welche Hindernisse könnten ihr im Wege stehen? Zwei Antworten bieten sich an. (i) Der Versicherungsvertrag ist nicht erzwingbar, da er illegal ist. Diese Antwort überzeugt nicht, da der Stpfl., der um die Zahlung $X^{*}$ gebracht werden soll, ein wirksames Druckmittel hat. Er weiß, daß sein Partner hinterzogen hat, und kann ihn anzeigen. (ii) Bevor eine Versicherung vereinbart werden kann, muß jeder der Beteiligten dem anderen mitteilen, daß er einen Steuerbetrug beabsichtigt oder bereits hinterzogen hat. Diese Mitteilung setzt natürlich ein gewisses Vertrauen voraus. Mangelt es daran, kommt die Versicherung nicht zustande.

Eine gegenseitige Versicherung von Steuerhinterziehern scheint ungewöhnlich zu sein. Gesetzesverstöße zeichnen sich jedoch oft durch die Kooperation von Gesetzesbrechern aus. Erwähnt sei hier nur das organisierte Verbrechen als extremste Form der Kooperation. Wichtige Gründe für diese Zusammenarbeit sind das Versicherungsmotiv und (mikroökonomisch ausgedrückt) produktionstechnische Vorteile. Die ökonomische Theorie der Kriminalität hat die Kooperation mehrerer Straftäter meines Wissens nach bisher nicht beachtet. In der Theorie gibt es nur den einsamen Gesetzesbrecher (siehe z.B. Pyle, 1983, Kapitel 2). Die Literatur zur Steuerhinterziehung macht da keine Ausnahme. Ich habe daher an einem Beispiel gezeigt, daß die Kooperation mikroökonomisch erklärt werden kann.

\subsection{Zusammenfassung}

Dieses Kapitel hat eine Vielzahl von Modifikationen und Erweiterungen des Grundmodells dargestellt. Die Ergebnisse sollen hier selektiv zusammengefaßt werden: 
Nur die Wirkungen eines höheren Einkommensteuersatzes auf die Steuerhinterziehung werden noch einmal festgehalten. Dieser Zusammenhang hat in der Literatur seit Allingham/Sandmo (1972) wohl aus folgendem Grund eine zentrale Rolle gespielt. Änderungen des Einkommensteuertarifs sind in vielen Ländern ein bevorzugtes Arbeitsfeld der Finanzpolitik, so daß die Vorhersage der Steuerwirkungen interessant wird. Andere für einen Steuerhinterzieher bedeutsame Parameter wie die Häufigkeit von Prüfungen und die Höhe der Strafen unterliegen zwar ebenfalls Änderungen - über diese spricht man in der finanzpolitischen Diskussion jedoch nicht so gerne.

In allen Modellen (angefangen mit dem Grundmodell) hat eine höhere Einkommensteuer einen negativen Einkommenseffekt auf das hinterzogene Einkommen, wenn man von einer abnehmenden absoluten Risikoaversion ausgeht. Varianten und Erweiterungen des Grundmodells enthalten zusätzliche Effekte, die zum Teil in die gleiche Richtung wie der Steuersatz weisen. Eine Kompensation des negativen Einkommenseffektes, so daß der oft vermutete und zum Teil empirisch bestätigte positive Zusammenhang zwischen Steuerbelastung und Abgabenwiderstand (vgl. Clotfelter, 1983, Poterba, 1987) zustande kommt, ist damit zwar möglich, aber nicht gesichert, wenn man nicht sehr spezielle Nutzenfunktionen annehmen will.

Lassen wir die Effekte, die für ein steigendes hinterzogenes Einkommen sprechen, Revue passieren. Ist statt der hinterzogenen Steuer das hinterzogene Einkommen Bemessungsgrundlage der Geldstrafe, kann ein höherer Steuersatz mit einem positiven Substitutionseffekt verbunden sein, da die Strafe pro DM nicht gezahlter Steuern sinkt. Mit der Strafe nach deutschem Recht sinkt der Preis der Steuerhinterziehung ebenfalls: Der Tagessatz ist von dem sicheren Nettoeinkommen abhängig, das durch die höhere Steuer vermindert wird. Sieht ein Hinterzieher, daß neben ihm noch viele andere seines Schlages existieren, und berücksichtigt er die entsprechende Kürzung öffentlicher Leistungen, kann es zu einer steigenden Hinterziehung kommen. Wenn sich ehrliche Steuerzahler von den Steuerhinterziehern anstecken lassen, führt eine höhere Steuerbelastung zu einer steigenden Anzahl von Hinterziehern, so daß die Summe aller nicht angegebenen Einkommen auf diesem Wege steigen kann.

Würde man alle Erweiterungen und Modifikationen des Grundmodells zusammenziehen, die einen positiven Zusammenhang zwischen Steuersatz und Steuerhinterziehung erklären können, könnte man mit Gordon (1989, S. 804) folgende Rechnung aufmachen: Viele Effekte sprechen für, aber nur ein Effekt gegen einen mit dem Steuersatz steigenden Abgabenwiderstand. Folglich sei dieser Zusammenhang "likely, if not conclusive". Leider reicht die Mathematik nicht aus, um eine Summe zu berechnen, wenn man nur die verschiedenen Vorzeichen der einzelnen Summanden kennt. Ein Stück Überzeugung gehört auch dazu. 


\section{Kapitel 3}

\section{Hinterziehung von Zinseinkommen}

\subsection{Einleitung}

Über Steuerhinterziehung wird in der Bundesrepublik zumeist unter zwei Stichworten diskutiert: "Schwarzarbeit" und "Nicht-Versteuerung von Zinsen". Zu dem ersten dieser zwei Themen enthält bereits Kapitel 2 einige Überlegungen. Dort ist auch darauf hingewiesen worden, daß viele Haushalte nicht die Möglichkeit haben, ihr Arbeitseinkommen zu hinterziehen, da es von der Lohnsteuer an der Quelle erfaßt wird. Jeder Haushalt jedoch, der einen Teil seines Arbeitseinkommens oder aus anderen Quellen spart, hat die Gelegenheit, Kapitaleinkommen zu hinterziehen, wenn er seine Ersparnisse in bestimmte festverzinsliche Kapitalanlagen investiert. Zinserträge werden im Wege der Veranlagung steuerlich erfaßt. Während des ersten Halbjahrs 1989 wurde ergänzend eine zehnprozentige Quellensteuer auf (viele) Inlandsanlagen erhoben, die sich der Sparer bei der Veranlagung für 1989 anrechnen lassen kann. Nur wenn Ersparnisse in Aktien angelegt werden, ergibt sich eine ähnliche Situation wie bei Löhnen und Gehältern aus regulären Beschäftigungsverhältnissen: 52 \% beträgt die "Quellensteuer" (Körperschaft- plus Kapitalertragsteuer) auf Dividenden, die sich die Anleger mit einem Grenzsteuersatz von weniger als $52 \%$ im Rahmen ihrer Einkommensteuerveranlagung anrechnen lassen. Die bevorzugte Sparform der Bundesbürger ist jedoch nicht das Risikokapital, sondern sind festverzinsliche Anlagen und Lebensversicherungen.

$\mathrm{Ob}$ man bei den Zinserträgen nur von einer Gelegenheit oder von einer Einladung zur Steuerhinterziehung sprechen sollte, ist eine Frage, die sich durch den "Bankenerlaß" aufdrängt. Dieser Erlaß, ursprünglich eine Verwaltungsanweisung an die Finanzbehörden, ist unter dem Titel "Schutz von Bankkunden" als $\S 30 \mathrm{a}$ in die Abgabenordnung übernommen worden. Er untersagt, Stichproben von Spar- 
konten zu erheben ( $\S 30$ a Abs. $2 \mathrm{AO}$ ), und soll die Betriebsprüfer der Banken vergessen lassen, was sie bei ihrer Arbeit über die Zinserträge privater Haushalte erfahren ( $\$ 30$ a Abs. $3 \mathrm{AO}$ ). Diese insbesondere im Vergleich zu den Lohneinkommen und Dividenden ungewöhnliche Behandlung der Zinsen hat in der wirtschaftspolitischen Diskussion seit langem zu Fragen geführt: Kann man von einer gleichmäßigen Besteuerung aller Einkommen - d.h. von einer Einkommensteuer - sprechen? Kann der Staat den Aufkommensverlust hinnehmen, der durch die Hinterziehung der Steuern auf Zinserträge entsteht? Welche Maßnahmen sind geeignet, um die Hinterziehung der Zinserträge einzudämmen?

Statt Stimmen aus dieser Diskussion zu hören, wollen wir einen Blick auf die Fakten werfen. Es gibt verschiedene Versuche, den Anteil der hinterzogenen Zinsen an den gesamten Zinserträgen der bundesdeutschen Haushalte zu schätzen (vgl. Krischausky, 1988, S. 96 f.). Die neueste und gründlichste Schätzung stammt von Krischausky (1988). Er hat die Geldvermögenseinkommen des Sektors "Private Haushalte", die die Deutsche Bundesbank berechnet, und die nach der Einkommensteuerstatistik des Statistischen Bundesamtes versteuerten Einkünfte aus Kapitalvermögen verglichen und dabei beachtet, daß (vgl. Krischausky, 1988, S. 85 - 118, hier S. 101)

a) ein Teil der Geldvermögenseinkünfte des Sektors "Private Haushalte" der Bundesbankstatistik Organisationen ohne Erwerbscharakter zuzurechen sind,

b) Zinseinkünfte aus Lebensversicherungsverträgen überwiegend steuerfrei sind,

c) für einen Teil der nicht per se steuerfreien Zinsen aus verschiedenen Gründen keine Steuer anfällt. Es seien hier nur die Stichworte Sparerfreibetrag, Webungskostenpauschale, Freigrenzen für Nebeneinkünfte, Personen mit einem Gesamteinkommen unterhalb des Grundfreibetrages genannt.

Die neueste Einkommensteuerstatistik, die Krischausky vorgelegen hat, bezieht sich auf das Jahr 1983. Für dieses Jahr kommt er zu der in Abbildung 3.1 wiedergegebenen Schätzung (Krischausky, 1988, S. 118). Der "Kuchen", den die Abbildung zeigt, besteht aus allen Geldvermögenseinkünften privater Haushalte in 1983 [81,7 Mrd. DM]. $31 \%$ [25 Mrd.] dieser Einkünfte waren steuerbefreit (Kategorie b). Für 29 \% [24 Mrd.] mußte aus anderen Gründen keine Einkommensteuer gezahlt werden (Kategorie c). Dabei ist die Frage, ob diese Zinserträge deklariert wurden oder nicht, unbedeutend, da daraus keine zusätzliche Steuerschuld resultiert hätte. $24 \%$ [19,5 Mrd.] aller Zinsen wurden angegeben und führten gleichzeitig zu einer zusätzlichen Steuerlast in Höhe von $8 \%$ [6,2 Mrd.] aller Zinserträge. $16 \%[13,2$ Mrd.] aller Zinsen wurden nicht deklariert und waren gleichzeitig mit einer Steuerersparnis verbunden (sofern keine spätere Entdeckung erfolgt ist). Wenn man als hinterzogene Einkünfte - im Unterschied zu nicht-deklarierten Einkünften - nur 


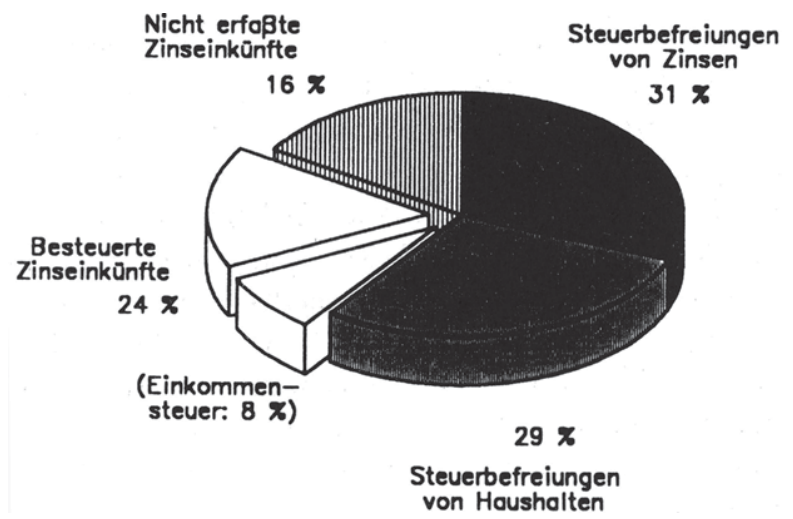

die Einkünfte bezeichnet, deren Nicht-Deklaration (zunächst) zu einer Steuerersparnis führt (vgl. Krischausky, 1988, S. 100 f.), betrug die Hinterziehungsquote $198316 \%$ und nicht 50 bis $90 \%$, wie andere Autoren vermutet oder geschätzt hatten (vgl. Krischausky, 1988, S. 3 und S. 97). Nicht die Steuerhinterziehung, sondern das Einkommensteuerrecht selbst ist die primäre Ursache für die Aushöhlung des Postulates der allgemeinen Steuerpflicht von Einkünften privater Haushalte aus Kapitalvermögen ( $\S 2$ Abs. 1 und 20 Abs. 1 EStG) (vgl. Krischausky, 1988, S. 198). Interessant ist noch folgender Vergleich: Das Aufkommen der Einkommensteuer (inclusive Lohnsteuer) betrug 1983 161,2 Mrd. DM (Statistisches Bundesamt, 1989). 6 Mrd. DM Aufkommensverlust durch die Hinterziehung von Zinsen, die Krischausky (1988, S. 116) für 1983 angibt, sind immerhin 3,7 \% dieser Summe.

Die Hinterziehung von Zinserträgen ist quantiativ weniger bedeutsam, als von der Politik und den Bürgern angenommen worden ist. Dennoch hat es im vergangenen Jahr den Versuch des Staates gegeben, sich mit Hilfe einer Quellensteuer an den von Jahr zu Jahr steigenden Zinserträgen der privaten Haushalte zu beteiligen. Es ist zu Ausweichreaktionen der von der neuen Steuer betroffenen und selbst der nicht betroffenen Sparer gekommen, und es wurde über alternative Maßnahmen zur Erfassung der Zinserträge diskutiert.

Die Frage, die sich hier stellt, ist, welchen Beitrag die Theorie der Steuerhinterziehung zu dieser Debatte leisten kann. Bei der Antwort auf diese Frage sollen zwei Fälle unterschieden werden, je nachdem ob die Wahrscheinlichkeit, daß eine 
Hinterziehung von Zinserträgen entdeckt wird, gleich null oder größer als null ist. Im ersten Fall benötigt man die positive Theorie der Steuerhinterziehung nicht. Ein Steuerpflichtiger, der nur an seinem Nettoeinkommen interessiert ist, hinterzieht seine Einkünfte soweit wie möglich, wenn er eine Entdeckung und Strafe nicht fürchten muß. Ist die Entdeckungswahrscheinlichkeit größer null, wird die Anwendung der Modelle eines Steuerhinterziehers interessant. Subjektiv kann jeder Stpfl. der Ansicht sein, seine Vergehen könnten entdeckt werden. Die objektive Wahrscheinlichkeit, bei der Hinterziehung oder Nicht-Deklaration von Zinsen entdeckt zu werden, ist für den kleinen Sparer vielleicht sehr gering. Bei Haushalten mit hohem Einkommen ist dagegen trotz des Bankenerlasses nicht davon auszugehen, daß eine Entdeckung unmöglich ist. $\S 30$ a $\mathrm{AO}$ schützt die Bankkunden dann nicht mehr, wenn diese Vorschrift "der finanzbehördlichen Verpflichtung zur Sachverhaltsaufklärung zuwiderläuft" (Bilsdorfer, 1989, S. 85) - und die Entstehung von Hinweisen auf den Sachverhalt einer Steuerstraftat oder -ordnungswidrigkeit läßt sich nicht ausschließen.

In der Praxis wird es beide Fälle geben: Der eine Sparer rechnet mit einer positiven, andere mit einer Entdeckungswahrscheinlichkeit von Null. Krischausky (1988, S. 126) geht davon aus, daß sie "nahe Null ist". Die Deklarationsentscheidungen der Sparer werden in seiner Arbeit über die "Besteuerung der Zinseinkünfte privater Haushalte" nicht entscheidungstheoretisch analysiert, so daß eine Erklärung für die positive Deklarationsquote von $24 \%$ aller Zinsen nicht zu finden ist. Er konzentriert sich auf empirische Aspekte der Zinsbesteuerung. In dieser Arbeit wird dagegen von einer positiven Entdeckungswahrscheinlichkeit ausgegangen und die Deklarationsentscheidungen der Sparer entscheidungstheoretisch untersucht.

In den Modellen der Steuerhinterziehung spielt es mit einem exogenen Einkommen keine Rolle, wie dieses Einkommen genannt wird. Es kann sich auch um Zinserträge handeln. Kurzfristig ist ein exogenes Einkommen für die Analyse finanzpolitischer Parameteränderungen eine sinnvolle Annahme. In längerfristiger Perspektive ist jedoch davon auszugehen, da $B$ die "Einladung zur Steuerhinterziehung" einen Einfluß auf die Sparentscheidung und die daraus resultierenden Zinserträge hat - und dies wiederum die Deklarationsentscheidung des Sparers beeinflußt. Die Kapitel 3 - 5 behandeln diesen Zusammenhang. In ihnen wird das Zwei-Perioden-Modell eines Sparers, das in vielen Bereichen der Mikroökonomie Anwendung gefunden hat, mit der Steuerhinterziehung verknüpft. Dabei werden die Zinserträge in den Kapiteln 3 und 4 einer Einkommensteuer und in Kapitel 4 zusätzlich einer Quellensteuer unterworfen. In Kapitel 5 ersetzt der Konsum das Einkommen als Bemessungsgrundlage der direkten Besteuerung, und es wird untersucht, welche Wirkungen sich durch diesen Wechsel ergeben.

Dieses Kapitel gliedert sich wie folgt. In Abschnitt 3.2 wird ein Modell mit en- 
dogenen Zinserträgen formuliert, das eine Anwendung des Zwei-Perioden-Modells eines Sparers mit einer sicheren und einer unsicheren Anlagemöglichkeit ist (siehe Sandmo, 1974, für einen Überblick). Es geht davon aus, daß die Arbeitsangebotsentscheidung des Haushalts bereits gefallen ist, und stellt damit das Analogon zu den in Kapitel 2 referierten Modellen mit endogenem Arbeitsangebot und exogener Sparentscheidung dar. Die Auswertung des Modells enthalten die Abschnitte 3.3 und 3.4. Es geht dabei um die Frage, in wieweit sich die komparativ-statischen $\mathrm{Zu}$ sammenhänge, die man bei einem exogenen Einkommen erhält, für ein endogenes Zinseinkommen verallgemeinern lassen. Abschnitt 3.5 faßt die Ergebnisse bei proportionalem Einkommensteuertarif und einer Geldstrafe als konstantem Vielfachen der hinterzogenen Steuer zusammen. In den Abschnitten 3.6 und 3.7 werden diese zwei Annahmen durch die Strafe nach deutschem Recht und einen progressiven Tarif ersetzt.

\subsection{Das Modell}

Der Stpfl. lebt zwei Perioden. In jeder Periode erhält er ein exogenes Lohneinkommen von $w_{1}$ bzw. $w_{2}$. Nach Abzug der Lohnsteuer mit dem Satz $t$ bleibt ihm davon $l_{i}=(1-t) w_{i}, i=1,2$. Der Konsum in Periode 1 ist $C_{1}$. Seine Ersparnisse $l_{1}-C_{1}$ kann der Haushalt zu einem sicheren Zinssatz $r$ anlegen. Die Zinsen $r\left(l_{1}-C_{1}\right)$, die er in der zweiten Periode erhält, muß er versteuern, da das Einkommen Steuerbemessungsgrundlage ist. Am Anfang der zweiten Periode gibt der Haushalt daher eine Steuerklärung ab. Diese wird mit einer exogenen Wahrscheinlichkeit $p$ kontrolliert; mit $q=1-p$ entgeht der Haushalt der Steuerfahndung. Anschließend kann er das Einkommen der Periode 2 nach Steuer und Strafe für Konsumausgaben verwenden.

Der Einkommensteuersatz ist ebenfalls gleich $t$. Die Strafe für die Steuerhinterziehung $\Phi_{1}=\alpha t H$ ist ein Vielfaches der hinterzogenen Steuer. Hat der Stpfl. Glück, ist sein Konsum in Periode 2

$$
C_{2 \mid \text { keineKontrolle }}:=G=l_{2}+(1+r)\left(l_{1}-C_{1}\right)-t\left(r\left(l_{1}-C_{1}\right)-H\right)
$$

$H$ ist das hinterzogene Zinseinkommen. Der letzte Summand ist die der Steuererklärung folgende Zahlung an das Finanzamt. Sie wird von dem Lohn $w_{2}$ nicht beeinflußt, da die Einkommensteuer auf Lohneinkommen mit der Lohnsteuer abgegolten ist. Mit der weiteren Abkürzung $R=1+(1-t) r$ lautet die Budgetbeschränkung

$$
G=l_{2}+R\left(l_{1}-C_{1}\right)+t H
$$

Wird der Haushalt steuerlich geprüft, ist der Konsum in Periode 2

$$
C_{2 \mid \text { Kontrolle }}:=P=l_{2}+(1+r)\left(l_{1}-C_{1}\right)-t\left(r\left(l_{1}-C_{1}\right)-H\right)-(1+\alpha) t H
$$




$$
=l_{2}+R\left(l_{1}-C_{1}\right)-\alpha t H
$$

Zielfunktion des Stpfl. ist sein Erwartungsnutzen. Die intertemporale Nutzenfunktion habe die Form $V\left(C_{1}\right)+W\left(C_{2}\right)$ mit $V^{\prime}, W^{\prime}>0, V^{\prime \prime}, W^{\prime \prime}<0$ Für die Wahl der additiv separablen Form gibt es zwei Gründe. Erstens soll die Vergleichbarkeit mit den Modellen von Pencavel (1979) und Wiegard (1984) mit endogenem Arbeitsangebot gewährleistet werden, die ebenfalls diese Form verwenden. Zweitens gibt es keine überzeugende Antwort bezüglich des Vorzeichens der Kreuzableitung einer intertemporalen Nutzenfunktion. Ich ziehe es daher vor, mit einer Kreuzableitung von null zu arbeiten. Diese Nutzenfunktion hat folgende Eigenschaften: Die Grenzrate der Substitution zwischen $C_{1}$ und $C_{2}$ ist negativ und nimmt ab. Weiter ist nach Keeney $\left(1973\right.$, S. 28 f.) $C_{2}$ "risk independent" von $C_{1}$ (und umgekehrt): Das Risiko einer Lotterie, die über $C_{1}$ und $C_{2}$ definiert ist, in der aber nur $C_{2}$ Zufallsvariable ist, ist unabhängig von festen Beträgen von $C_{1}$, wenn man das Risiko durch die absolute Risikoaversion mißt.

Es gilt $E\left(V\left(C_{1}\right)+W\left(C_{2}\right)\right)=V\left(C_{1}\right)+q W(G)+p W(P)$. Das Entscheidungsproblem des Haushalts lautet damit

$$
\begin{array}{cl}
\max _{C, H} & V(C)+q W\left(l_{2}+R\left(l_{1}-C\right)+t H\right)+p W\left(l_{2}+R\left(l_{1}-C\right)-\alpha t H\right) \\
\text { u.d.N. } & 0 \leqq H \leqq r\left(l_{1}-C\right)
\end{array}
$$

$C_{1}$ ist mit $C$ abgekürzt. $C_{2}$ ist keine Entscheidungsvariable, da die Beschränkungen $G$ und $P$ in die Nutzenfunktion eingesetzt wurden.

Es wird angenommen, daß der Haushalt positive Beträge spart. Ein Stpfl., der sich verschuldet, hätte keine Gelegenheit, Zinserträge zu hinterziehen und ist für unsere Analyse nicht von Interesse. Zweitens wird angenommen, daß der Haushalt nicht das gesamte exogene Einkommen $l_{1}$ spart, sondern daß er in jeder Periode einen positiven Konsum realisieren will ${ }^{1}$. Ecklösungen in bezug auf den optimalen Konsum $C^{*}$ sind damit ausgeschlossen.

Die Bedingungen erster Ordnung für eine innere Lösung bezüglich $C$ und $H$ sind

$$
\begin{aligned}
& E_{C}=V^{\prime}\left(C^{*}\right)-R\left(q W^{\prime}(G)+p W^{\prime}(P)\right)=0 \\
& E_{H}=q W^{\prime}(G) t-p W^{\prime}(P) \alpha t=0
\end{aligned}
$$

$E_{C}$ bezeichnet die erste Ableitung der Erwartungsnutzenfunktion nach $C . E_{H}$ ist die Ableitung nach $H$. (3.1) ist die Bedingung für die optimale Höhe der Ersparnis. Sie besagt, daß die Grenzrate der Substitution des erwarteten zukünftigen Konsums durch den heutigen Konsum gleich dem intertemporalen Preisverhältnis

\footnotetext{
${ }^{1}$ Eine hinreichende Bedingung für dieses Verhalten ist, daB $V^{\prime}(0)$ und $W^{\prime}(0)$ sehr groB sind.
} 
$R$ zwischen $C_{1}$ und $C_{2}$ sein muß. Dieses Preisverhältnis wird von dem Zinssatz nach Steuer bestimmt. (3.2) ist die Bedingung für den optimalen Umfang des hinterzogenen Einkommens in Periode 2.

Aus (3.2) erhält man die Voraussetzungen für das Zustandekommen einer inneren Lösung $\left(0<H^{*}<r\left(l_{1}-C^{*}\right)\right)$ :

$$
\begin{aligned}
E_{H \mid H=0} & =W^{\prime}\left(C_{2}\right) t(q-p \alpha)>0 \\
E_{H \mid H=r\left(l_{1}-C\right)} & =q W^{\prime}(G) t-p W^{\prime}(P) \alpha t<0
\end{aligned}
$$

Faßt man beide Bedingungen zusammen, ergibt sich

$$
\left.\frac{W^{\prime}(P)}{W^{\prime}(G)}\right|_{H=r\left(l_{1}-C\right)}>\frac{q}{p \alpha}>1
$$

Da der Ausdruck auf der linken Seite größer eins ist, kann man Werte der Parameter $p$ und $\alpha$ finden, die diese Bedingung erfüllen. Für vorgegebene Werte von $p$ und $\alpha$, die $q-p \alpha>0$ erfüllen, ist jedoch nicht ausgeschlossen, daß der Stpfl. seine gesamten Zinserträge hinterzieht. Mit einer hohen erwarteten Rendite der Steuerhinterziehung kann die obere Ecklösung $H^{*}=r\left(l_{1}-C^{*}\right)$ auch für einen risikoaversen Haushalt optimal sein. Ich kommen daher nach der Analyse einer inneren Lösung auf die Ecklösung zu sprechen.

Hinreichende Bedingungen zweiter Ordnung für eine innere Lösung sind (vgl. Silberberg, 1978, S. 104)

$$
E_{C C}=V^{\prime \prime}\left(C^{*}\right)+R^{2}\left(q W^{\prime \prime}(G)+p W^{\prime \prime}(P)\right)<0
$$

und ein positives Vorzeichen der Determinante

$$
\begin{aligned}
\Delta= & \left|\begin{array}{ll}
E_{C C} & E_{C H} \\
E_{H C} & E_{H H}
\end{array}\right| \\
= & {\left[V^{\prime \prime}\left(C^{*}\right)+R^{2}\left(q W^{\prime \prime}(G)+p W^{\prime \prime}(P)\right)\right]\left[t^{2}\left(q W^{\prime \prime}(G)+p W^{\prime \prime}(P) \alpha^{2}\right)\right] } \\
& -\left[-t R\left(q W^{\prime \prime}(G)-p W^{\prime \prime}(P) \alpha\right)\right]^{2} \\
= & V^{\prime \prime}\left(C^{*}\right) E_{H H}+R^{2} t^{2}\left[\left(q W^{\prime \prime}(G)+p W^{\prime \prime}(P)\right)\left(q W^{\prime \prime}(G)+p W^{\prime \prime}(P) \alpha^{2}\right)\right. \\
& \left.-\left(q W^{\prime \prime}(G)-p W^{\prime \prime}(P) \alpha\right)^{2}\right]
\end{aligned}
$$

Der erste Summand ist größer null. Das Vorzeichen des zweiten ist von dem Ausdruck in der eckigen Klammer abhängig, der die Form

$$
(x+y)\left(x+y \alpha^{2}\right)-(x-y \alpha)^{2}
$$

mit $x, y<0$ hat. Ausmultiplizieren ergibt $x y(1+\alpha)^{2}>0$. Damit ist die Bedingung $\Delta>0$ erfüllt. 


\subsection{Komparative Statik bei einer inneren Lösung}

Teilt man die Optimalitätsbedingung (3.2) zur Vereinfachung durch $t$, erhält man

$$
E_{H}=q W^{\prime}(G)-p W^{\prime}(P) \alpha=0
$$

(3.1) und (3.3) bestimmen die Funktionen

$$
\begin{aligned}
C^{*} & =C^{*}\left(p, \alpha, t, r, w_{1}, w_{2}\right) \\
H^{*} & =H^{*}\left(p, \alpha, t, r, w_{1}, w_{2}\right)
\end{aligned}
$$

Setzt man diese Funktionen in (3.1) und (3.3) ein, erhält man die Identitäten

$$
\begin{aligned}
& E_{C}\left(C^{*}(\cdot), H^{*}(\cdot), \cdot\right) \equiv 0 \\
& E_{H}\left(C^{*}(\cdot), H^{*}(\cdot), \cdot\right) \equiv 0
\end{aligned}
$$

Die totale Differentiation nach einem Parameter $z$ ergibt

$$
\left(\begin{array}{ll}
E_{C C} & E_{C H} \\
E_{H C} & E_{H H}
\end{array}\right)\left(\begin{array}{l}
\partial C^{*} / \partial z \\
\partial H^{*} / \partial z
\end{array}\right)=\left(\begin{array}{c}
-E_{C z} \\
-E_{H z}
\end{array}\right)
$$

Die Reaktion des hinterzogenen Einkommens auf eine Erhöhung von $z$ ist

$$
\frac{\partial H^{*}}{\partial z}=-\tilde{\Delta}^{-1}\left(E_{C C} E_{H z}-E_{C z} E_{H C}\right)
$$

wobei $\tilde{\Delta}=\Delta / t$ ist.

Im folgenden soll es um zwei Fragen gehen. Wie wirken sich Kontrollen und Strafen auf die Hinterziehung von Zinserträgen aus? Zweitens wird die Frage beantwortet, wie eine Erhöhung des Zinsniveaus die Hinterziehung der Zinserträge beeinflußt.

\subsubsection{Strafen und Kontrollen bei endogenem Zinseinkommen}

Von verschiedenen Seiten ist vorgeschlagen worden, Stichproben von Konten zu erheben, um die Zinserträge steuerlich zu erfassen. Diese Maßnahme würde die Entdeckungswahrscheinlichkeit erhöhen, mit der der Sparer rechnet. Seine Reaktion darauf kann wie folgt abgeleitet werden:

$$
\frac{\partial H^{*}}{\partial p}=-\tilde{\Delta}^{-1}\left(E_{C C} E_{H p}-E_{C p} E_{H C}\right)
$$




$$
\begin{aligned}
= & -\tilde{\Delta}^{-1}\left[\left(V^{\prime \prime}\left(C^{*}\right)+R^{2}\left(q W^{\prime \prime}(G)+p W^{\prime \prime}(P)\right)\right.\right. \\
& \left(-W^{\prime}(G)-W^{\prime}(P) \alpha\right) \\
& \left.-R^{2}\left(-W^{\prime}(G)+p W^{\prime}(P)\right)\left(q W^{\prime \prime}(G)-p W^{\prime \prime}(P) \alpha\right)\right] \\
= & \tilde{\Delta}^{-1}\left[V^{\prime \prime}\left(C^{*}\right)\left(W^{\prime}(G)+W^{\prime}(P) \alpha\right)\right. \\
& \left.+R^{2}\left(q W^{\prime \prime}(G) W^{\prime}(P)+p W^{\prime \prime}(P) W^{\prime}(G)\right)(1+\alpha)\right]<0
\end{aligned}
$$

Eine höhere Entdeckungswahrscheinlichkeit ist ein wirksames Mittel gegen den Steuerbetrug. Eine Erhöhung des Strafsatzes $\alpha$ führt zu

$$
\begin{aligned}
\frac{\partial H^{*}}{\partial \alpha}= & -\tilde{\Delta}^{-1}\left[E_{C C} E_{H \alpha}-E_{C \alpha} E_{H C}\right] \\
= & -\tilde{\Delta}^{-1}\left[\left(V^{\prime \prime}\left(C^{*}\right)+R^{2}\left(q W^{\prime \prime}(G)+p W^{\prime \prime}(P)\right)\right)\right. \\
& \left(-p W^{\prime}(P)+p W^{\prime \prime}(P) \alpha t H^{*}\right) \\
& \left.-R p W^{\prime \prime}(P) t H^{*} R\left(-q W^{\prime \prime}(G)+p W^{\prime \prime}(P) \alpha\right)\right] \\
= & \tilde{\Delta}^{-1}\left[V^{\prime \prime}\left(C^{*}\right)\left(p W^{\prime}(P)-p W^{\prime \prime}(P) \alpha t H^{*}\right)\right. \\
& +R^{2} p W^{\prime}(P)\left(q W^{\prime \prime}(G)+p W^{\prime \prime}(P)\right) \\
& \left.-R^{2} q W^{\prime \prime}(G) p W^{\prime \prime}(P) t H(1+\alpha)\right]<0
\end{aligned}
$$

Eine isolierte Erhöhung des Strafsatzes reduziert ebenfalls die hinterzogenen Zinseinkommen.

Für das Modell mit exogenem Einkommen und der Strafe $\Phi_{1}$ ist in Kapitel 2 die Frage gestellt worden, ob Kontrollen oder Strafen die wirksameren Mittel sind, um die Steuerhinterziehung zu begrenzen. Diese Frage ist dort wie folgt präzisiert worden: Wie entwickelt sich $H^{*}$, wenn $\alpha$ erhöht und $p$ soweit gesenkt wird, daß der erwartete durchschnittliche Ertrag der Steuerhinterziehung, $q-p \alpha$, konstant (gleich $k>0$ ) bleibt? Die Antwort lautete: $H^{*}$ sinkt mit dieser Umgestaltung des Systems der Steuersicherung. Dieses Ergebnis erhält man auch dann, wenn die Annahme eines exogenen Einkommens aufgegeben wird. Mit Hilfe der zwei oben abgeleiteten partiellen Ableitungen von $H^{*}$ nach $\alpha$ und $p$ und $\partial p / \partial \alpha=-p /(1+\alpha)$ ergibt sich

$$
\begin{aligned}
& \left.\frac{\partial H^{*}}{\partial \alpha}\right|_{q-p \alpha=k}=\frac{\partial H^{*}}{\partial \alpha}+\frac{\partial H^{*}}{\partial p} \frac{\partial p}{\partial \alpha} \\
& =\tilde{\Delta}^{-1}\left[-V^{\prime \prime}\left(C^{*}\right) p W^{\prime \prime}(P) \alpha t H^{*}-V^{\prime \prime}\left(C^{*}\right)\left(W^{\prime}(G)-W^{\prime}(P)\right) \frac{p}{1+\alpha}\right. \\
& -R^{2} p^{2} W^{\prime \prime}(P)\left(W^{\prime}(G)-W^{\prime}(P)\right) \\
& \left.-R^{2} q W^{\prime \prime}(G) p W^{\prime \prime}(P) t H^{*}(1+\alpha)\right]<0
\end{aligned}
$$

Ob man Steuerhinterzieher jedoch mit der Wahrscheinlichkeit "null" hängen sollte, ist mit diesem Resultat nicht beantwortet. Ex ante werden zwar auch mit einer 
kleinen Entdeckungswahrscheinlichkeit und einer großen Strafe alle Stpfl. gleich behandelt. Ex post aber stehen in diesem Fall die entdeckten und die erfolgreichen Hinterzieher sehr unterschiedlich da. Diese Statuierung eines Exempels dürfte von vielen Steuerzahlern als Verstoß gegen die Norm der steuerlichen Gleichbehandlung von Gleichen empfunden werden (vgl. Skinner/Slemrod, 1985, S. 348 f.). Für einen "Erwartungsnutzenmaximierer", der das Risiko kennt, das mit der Hinterziehung verbunden ist, wäre es allerdings unglaubwürdig, ex post über eine mangelnde Gleichbehandlung zu klagen, da er die Konsequenzen seines Tuns ex ante in Kauf nimmt.

Wenn es nicht opportun ist, die Strafe zu erhöhen, bietet sich eine Intensivierung der Prüfungen an, wenn die Steuerhinterziehung eingedämmt werden soll. Die verkürzte Steuer ist jedoch nicht die einzige Größe, die für die Finanzpolitik bedeutsam ist. Insbesondere bei einer angespannten Finanzlage der öffentlichen Hand ist auch die Entwicklung des Steueraufkommens von Interesse. Betrachten wir daher, wie sich das erwartete Aufkommen aus der Besteuerung von Zinsen

$$
A=\operatorname{tr}\left(l_{1}-C^{*}\right)-(q-p \alpha) t H^{*}
$$

mit $p$ ändert:

$$
\frac{\partial A}{\partial p}=-\operatorname{tr} \frac{\partial C^{*}}{\partial p}+(1+\alpha) t H^{*}-(q-p \alpha) t \frac{\partial H^{*}}{\partial p}
$$

Die beiden letzten Summanden sind positiv. Die Änderung des Konsums läßt sich jedoch nicht bestimmen. Auch der Nettoeffekt auf das Steueraufkommen ist ungewiß (siehe Anhang 3.3.1 ${ }^{2}$ ). Dieses Ergebnis kann man noch auf einem anderen Weg ableiten. Schreibt man das Aufkommen in der Form

$$
A=t D^{*}+p(1+\alpha) t H^{*}
$$

wobei $D^{*}=r\left(l_{1}-C^{*}\right)-H^{*}$ das deklarierte Einkommen ist, und differenziert nach $p$, ergibt sich

$$
\frac{\partial A}{\partial p}=t \frac{\partial D^{*}}{\partial p}+(1+\alpha) t H^{*}+p(1+\alpha) t \frac{\partial H^{*}}{\partial p}
$$

Das deklarierte Einkommen steigt mit $p$ (Anhang 3.3.1). Da $H^{*}$ sinkt und der letzte Summand damit negativ ist, kommt es auf das Verhältnis der Änderungen des deklarierten und des hinterzogenen Einkommens an. Über diese Relation läßt sich jedoch im allgemeinen nichts sagen.

\footnotetext{
${ }^{2}$ Ein Teil der Berechnungen und Ergebnisformeln zu diesem Kapitel findet sich in Anhang 3.
} 


\subsubsection{Zinsniveau und die Hinterziehung von Kapitalerträgen}

Es bleibt zu untersuchen, welchen Einfluß die übrigen Parameter des Modells haben. In bezug auf den Steuersatz enthält Abschnitt 3.5 kurze Bemerkungen. Ich konzentriere mich hier auf die Wirkung des Zinssatzes auf die Hinterziehung, da dieser Parameter in dem Grundmodell keine Entsprechung hat.

In den vergangenen zwei Jahrzehnten war das Zinsniveau in den westlichen Volkswirtschaften zunehmenden Schwankungen unterworfen (Strange, 1986, S. 14 - 17). Unter den vielfältigen Konsequenzen von Variationen des Zinssatzes ist sein Einfluß auf die Steuerehrlichkeit der Sparer unbeachtet geblieben. Eine erste Antwort über diesen Zusammenhang kann man dem Partialmodell dieses Abschnitts entnehmen.

$$
\begin{aligned}
\frac{\partial H^{*}}{\partial r}= & -\tilde{\Delta}^{-1}(1-t) q W^{\prime}(G)\left(R_{a}(G)-R_{a}(P)\right) \\
& {\left[-V^{\prime \prime}\left(C^{*}\right)\left(l_{1}-C^{*}\right)+R\left(q W^{\prime}(G)+p W^{\prime}(P)\right)\right]>0 }
\end{aligned}
$$

wobei $R_{a}(C)=-W^{\prime \prime}\left(C_{2}\right) / W^{\prime}\left(C_{2}\right), C_{2}=P, G$, das Maß der absoluten Risikoaversion in bezug auf den Konsum in Periode 2 ist. Mit einer abnehmenden absoluten Risikoaversion hinterzieht der Haushalt mehr Zinserträge, wenn der Zinssatz steigt. Dieses Verhalten könnte man wie folgt begründen. Der höhere Zins stimuliert die Ersparnis. Mit einem gestiegenen Kapitaleinkommen in Periode 2 sinkt die absolute Risikoaversion des Stpfl., so daß er mehr Steuern hinterzieht. Diese Argumentation hat leider einen schwachen Punkt. Aus dem Modell ergibt sich nämlich

$$
\begin{aligned}
\frac{\partial C^{*}}{\partial r}= & \tilde{\Delta}^{-1}(1-t) t q^{2} W^{\prime}(G)^{2}(1+1 / \alpha) \times \\
& {\left[R_{a}(G)+R_{a}(P)+R R_{a}(P)\left(R_{a}(G)-R_{a}(P)\right)\right] \lesseqgtr 0 }
\end{aligned}
$$

Der Konsum (die Ersparnis) kann also auch mit dem Zins steigen (sinken). Es ist daher zunächst überraschend, daß der höhere Zins die Hinterziehung der entsprechenden Erträge selbst dann stimuliert, wenn die Ersparnis $l_{1}-C^{*}$ abnimmt.

Die Zerlegung von (3.4) und (3.5) mit Hilfe von Hicks-Slutsky-Gleichungen ist hilfreich, um dieses mögliche Ergebnis zu verstehen. $G$ und $P$ werden um einen Transfer $a$ ergänzt, über den die Wirkung der Änderung des Zinssatzes auf das Nutzenniveau der Ausgangslage kompensiert wird. Es gilt

$$
\frac{\partial H^{*}}{\partial r}=\frac{\partial H^{+}}{\partial r}-\frac{\partial a^{+}}{\partial r} \frac{\partial H^{*}}{\partial a}
$$

und

$$
\frac{\partial C^{*}}{\partial r}=\frac{\partial C^{+}}{\partial r}-\frac{\partial a^{+}}{\partial r} \frac{\partial C^{*}}{\partial a}
$$


wobei $\mathrm{H}^{+}$und $\mathrm{C}^{+}$die einkommenskompensierten Nachfragen sind und $a^{+}$die Ausgabenfunktion ist (vgl. Abschnitt 2.1.1). Ich berechne die Substitutions- und Einkommenseffekte mit Hilfe des indirekten Verfahrens, das bereits in Kapitel 2 Anwendung fand, statt das Ausgabenminimierungsproblem explizit zu lōsen. Die Kompensation

$$
\frac{\partial a^{+}}{\partial r}=-\frac{\partial E^{i} / \partial r}{\partial E^{i} / \partial a}=-(1-t)\left(l_{1}-C^{*}\right)
$$

erhält man durch Differentiation der indirekten Nutzenfunktion (vgl. Abschnitt 2.1.1). Der sinkende Transfer $a^{+}$bewirkt, daß das Nutzenniveau der Ausgangslage erhalten bleibt.

Die Einkommenseffekte können nun leicht bestimmt werden. Aus dem primalen Problem erhält man $\partial H^{*} / \partial a>0$ und $1 / R>\partial C^{*} / \partial a>0$ (Anhang 3.3.2). Mit einem höheren Transfer in Periode 2 steigen der Konsum in Periode 1 und der erreichbare sichere Konsum in Periode $2, l_{2}+R\left(l_{1}-C^{*}\right)+a$. Der Haushalt teilt ein höheres exogenes Einkommen auf beide Perioden auf. Nimmt die absolute Risikoaversion mit dem erreichbaren sicheren Konsum ab, steigt das hinterzogene Einkommen. Die Multiplikation der Ableitungen von $H^{*}$ und $C^{*}$ mit $-\left(-(1-t)\left(l_{1}-C^{*}\right)\right)$ ergibt jeweils einen positiven Einkommenseffekt des Zinssatzes. Die Substitutionseffekte erhält man, indem man die Einkommens- von den Gesamteffekten abzieht. Die Reaktion des einkommenskompensierten Konsums ist:

$$
\frac{\partial C^{+}}{\partial r}=\tilde{\Delta}^{-1} E_{H H}(1-t)\left(q W^{\prime}(G)+p W^{\prime}(P)\right)<0
$$

Es lohnt sich (bei gegebenem Erwartungsnutzen), mehr zu sparen; der Preiseffekt auf die Ersparnis ist positiv. Das sichere Einkommen in Periode 2 steigt nun, da der sinkende Transfer $a^{+}$nur die Wirkung des höheren Zinssatzes bei gegebener Ersparnis kompensiert:

$$
\frac{\partial\left(l_{1}+R\left(l_{1}-C^{+}\right)+a^{+}\right)}{\partial r}=-R \frac{\partial C^{+}}{\partial r}
$$

Mit dem höheren sicheren Nettoeinkommen in Periode 2 steigt die kompensierte "Nachfrage" nach dem hinterzogenen Einkommen (Anhang 3.3.2):

$$
\frac{\partial H^{+}}{\partial r}=\tilde{\Delta}^{-1} V^{\prime \prime}(C) q W^{\prime}(G)\left(R_{a}(G)-R_{a}(P)\right)>0
$$

Addiert man die Substitutions- und Einkommenseffekte auf das hinterzogene Einkommen und den Konsum, erhält man die positive Änderung der normalen Nachfrage $H^{*}$ und die unbestimmte Reaktion der Ersparnis $l_{1}-C^{*}$ (Gleichungen (3.4) und (3.5)). Selbst wenn also die Ersparnis mit $r$ sinkt, erhöht der Stpfl. das hinterzogene Einkommen, da das (erreichbare) sichere verzinste Geldvermögen nach 
Steuer zu Beginn von Periode 2 mit $r$ steigt:

$$
\begin{aligned}
\frac{\partial R\left(l_{1}-C^{*}\right)}{\partial r} & =(1-t)\left(l_{1}-C^{*}\right)-R\left(\frac{\partial C^{+}}{\partial r}-\frac{\partial a^{+}}{\partial r} \frac{\partial C^{*}}{\partial a}\right) \\
& =(1-t)\left(l_{1}-C^{*}\right)\left(1-R \frac{\partial C^{*}}{\partial a}\right)-R \frac{\partial C^{+}}{\partial r}>0
\end{aligned}
$$

Die Ersparnisse sinken zwar infolge des negativen Einkommenseffektes; der steigende Zins kompensiert jedoch dessen Wirkung auf die Größe $R\left(l_{1}-C^{*}\right)$.

Will man die Steuerehrlichkeit des Haushalts durch das deklarierte Einkommen $D^{*}=r\left(l_{1}-C^{*}\right)-H^{*}$ statt durch $H^{*}$ messen, stellt sich die Frage nach dem Vorzeichen $\partial D^{*} / \partial r$. Die Zinserträge $r\left(l_{1}-C^{*}\right)$ steigen auch dann mit $r$, wenn $\partial C^{*} / \partial r$ negativ ist. $\partial H^{*} / \partial r$ ist ebenfalls positiv. Über die relative Stärke der zwei Änderungen kann man jedoch allein mit der Annahme einer abnehmenden absoluten Risikoaversion keine Aussage machen. Der Einfluß eines höheren Zinssatzes auf das deklarierte Einkommen ist daher unbestimmt. Auch über die Entwicklung des erwarteten Aufkommens aus der Besteuerung der Zinserträge

$$
A=\operatorname{tr}\left(l_{1}-C^{*}\right)-(q-p \alpha) t H^{*}
$$

läßt sich keine eindeutige Aussage machen. Die Zinserträge $r\left(l_{1}-C^{*}\right)$ steigen zwar mit $r$. Gleichzeitig steigt jedoch auch der erwartete Ertrag der Hinterziehung $(q-p \alpha) t H^{*}$.

\subsection{Komparative Statik bei einer Ecklösung}

Unter der Voraussetzung $q-p \alpha>0$ wird der Stpfl. unabhängig von dem Ausmaß seiner Risikoaversion zu einem Steuerhinterzieher, wie wir oben gesehen haben. Wie weit der Stpfl. jedoch in der Verkürzung der Steuern geht, kann man im allgemeinen nicht beantworten. Möglicherweise hinterzieht er nur einen Teil seiner Zinserträge. Wenn der erwartete Ertrag der Hinterziehung groß ist, wird es aber auch Stpfl. geben, die die Ecklösung $H^{*}=r\left(l_{1}-C^{*}\right)$ wählen.

Sei

$$
L=V(C)+q W(G)+p W(P)+\lambda\left(r\left(l_{1}-C\right)-H\right)
$$

die Lagrange-Funktion zu dem Optimierungsproblem des Stpfl. $\lambda$ ist der LagrangeMultiplikator der Nebenbedingung $H \leqq r\left(l_{1}-C\right)$. Die Ecklösung in bezug auf $H$ ist durch die Bedingungen erster Ordnung

$$
\begin{aligned}
& L_{C}=V^{\prime}\left(C^{*}\right)-(1+r(1-t))\left(q W^{\prime}(G)+p W^{\prime}(P)\right)-\lambda r=0 \\
& L_{H}=q W^{\prime}(G) t-p W^{\prime}(P) \alpha t-\lambda r=0 \\
& L_{\lambda}=r\left(l_{1}-C^{*}\right)-H^{*}=0
\end{aligned}
$$


charakterisiert, wobei $\lambda>0$ ist.

Ein Stpfl., der alle Zinsen hinterzieht, wird auch dann noch kein Kapitaleinkommen deklarieren, wenn sich einer der Parameter seines Entscheidungsproblems marginal ändert. Der Konsum $C^{*}$ kann jedoch variieren, so daß sich auch das hinterzogene Einkommen $H^{*}=r\left(l_{1}-C^{*}\right)$ ändert - und zwar jeweils in der entgegengesetzten Richtung, wenn $r$ und $l_{1}$ konstant bleiben. Es wird daher im folgenden untersucht, wie $C^{*}$ auf marginale Änderungen der Parameter reagiert.

Setzt man (3.8) und (3.7) in (3.6) ein, erhält man

$$
\begin{aligned}
& V^{\prime}\left(C^{*}\right)-(1+r) q W^{\prime}\left(l_{2}+(1+r)\left(l_{1}-C^{*}\right)\right) \\
& -(1+r(1-t(1+\alpha))) p W^{\prime}\left(l_{2}+(1+r(1-t(1+\alpha)))\left(l_{1}-C^{*}\right)\right)=0
\end{aligned}
$$

Diese Optimalitätsbedingung würde sich auch dann ergeben, wenn dem Stpfl. vorgegeben würde, alle Zinserträge zu hinterziehen: Die Verzinsung seiner Ersparnisse wäre dann ebenfalls gleich $r$ oder, wenn er weniger Glück hat, gleich $r(1-t(1+\alpha))$.

Die Ableitung von (3.9) nach $C^{*}$ ergibt

$$
\Delta=V^{\prime \prime}\left(C^{*}\right)+(1+r)^{2} q W^{\prime \prime}(G)+(1+r(1-t(1+\alpha)))^{2} p W^{\prime \prime}(P)<0
$$

Die implizite Differentiation von (3.9) nach $p$ führt nun zu:

$$
\frac{\partial C^{*}}{\partial p}=-\Delta^{-1}\left[(1+r) W^{\prime}(G)-(1+r(1-t(1+\alpha))) W^{\prime}(P)\right] \lesseqgtr 0
$$

Damit läßt sich auch über das Vorzeichen von $\partial H^{*} / \partial p=-r \partial C^{*} / \partial p$ nichts aussagen. Das gilt auch für den Fall, daß der Stpfl. die Entdeckungswahrscheinlichkeit als sehr gering einschätzt. Entsprechende Aussagen erhält man, wenn sich die zwei übrigen finanzpolitisch beeinflußbaren Parameter $\alpha$ und $t$ ändern (siehe Anhang 3.4).

Die Erhöhung des Strafsatzes $\alpha$, begleitet von einer Senkung der Entdeckungswahrscheinlichkeit, die $q-p \alpha$ konstant hält, läßt keine Vorhersage für einen Stpfl. zu, der keine Zinserträge versteuert. Die von der Analyse der inneren Lösung suggerierte Politik, die Strafen zu verschärfen und weniger auf Kontrollen zu setzen, findet durch die Analyse jener Gruppe keine Unterstützung (Anhang 3.4).

Eine Erhöhung des Zinssatzes führt zu:

$$
\begin{aligned}
\frac{\partial C^{*}}{\partial r}= & \Delta^{-1}\left[q W^{\prime}(G)+(1+r) \dot{q} W^{\prime \prime}(G)\left(l_{1}-C^{*}\right)+(1-t(1+\alpha)) p W^{\prime}(P)\right. \\
& \left.+(1+r(1-t(1+\alpha))) p W^{\prime \prime}(P)(1-t(1+\alpha))\left(l_{1}-C^{*}\right)\right] \lesseqgtr 0
\end{aligned}
$$

Die Veränderung des Konsums (bzw. der Ersparnis) bleibt unbestimmt. Dieses Ergebnis kann nicht überraschen, da schon in dem Zwei-Perioden-Modell ohne eine Besteuerung, in dem die Ersparnis eine sichere Rendite $r$ erzielt (siehe z.B. 
Sandmo, 1985, S. 266 - 269), dem negativen (bzw. positiven) Substitutionseffekt ein (bei normalen Gütern $C_{1}$ und $C_{2}$ ) positiver (bzw. negativer) Einkommenseffekt gegenübersteht (vgl. Sandmo, 1985, S. 268 f.). In dem vorliegenden Modell bedeutet eine Erhöhung von $r$ nur nicht einfach eine steigende sichere Rendite der Ersparnis, sondern eine steigende erwartete Rendite $r(q+p(1-t(1+\alpha)))$ (vgl. Dardanoni, 1988, S. 438). Diese Interpretation erklärt auch die unbestimmte Reaktion von $C^{*}$ und $H^{*}$ auf eine Erhöhung der Parameter $p, \alpha$ und $t$, die ebenfalls die erwartete Rendite der Anlagen eines Sparers beeinflussen, der alle Zinsen hinterzieht. Bei einer Veränderung des Zinssatzes kommt man jedoch immerhin zu der Aussage, daß

$$
\frac{\partial H^{*}}{\partial r}=\frac{\partial r\left(l_{1}-C^{*}\right)}{\partial r}=l_{1}-C^{*}-r \frac{\partial C^{*}}{\partial r}
$$

positiv ist (Anhang 3.4). Die Ersparnis sinkt in keinem Fall so weit, daß die hinterzogenen Zinserträge nicht steigen.

Bei einer Ecklösung kann man über die Entwicklung des Aufkommens aus der Besteuerung von Zinserträgen

$$
A=p(1+\alpha) \operatorname{tr}\left(l_{1}-C^{*}\right)
$$

nur etwas aussagen, wenn sich einer der Parametern ändert, die die Bruttoeinkommen des Stpfl. in den zwei Perioden bestimmen $\left(r, w_{1}\right.$ und $\left.w_{2}\right)$. Für den Zins sieht man das sofort: $\partial A / \partial r=p(1+\alpha) t \partial H^{*} / \partial r$. Eine Änderung der finanzpolitischen Parameter $p, \alpha$ und $t$ läßt dagegen unbestimmt, ob das Aufkommen steigt. Für die Entdeckungswahrscheinlichkeit soll das hier gezeigt werden:

$$
\begin{aligned}
\frac{\partial A}{\partial p}= & (1+\alpha) \operatorname{tr}\left[\left(l_{1}-C^{*}\right) \frac{\Delta}{\Delta}-p r \frac{\partial C^{*}}{\partial p}\right] \\
= & \frac{(1+\alpha) \operatorname{tr}}{\Delta}\left[( l _ { 1 } - C ^ { * } ) \left\{V^{\prime \prime}\left(C^{*}\right)+(1+r)^{2} q W^{\prime \prime}(G)\right.\right. \\
& \left.+(1+r(1-t(1+\alpha)))^{2} p W^{\prime \prime}(P)\right\} \\
& \left.+p\left\{(1+r) W^{\prime}(G)-(1+r(1-t(1+\alpha))) W^{\prime}(P)\right\}\right] \lesseqgtr 0
\end{aligned}
$$

Im Fall einer Ecklösung lassen damit gerade die Parameter, die von finanzpolitischem Interesse sind, keine eindeutigen Aussagen über das Verhalten des Steuerhinterziehers und die Entwicklung des Steueraufkommens zu.

\subsection{Ergebnisse bei proportionalem Tarif und der Strafe $\Phi_{1}$}

In den Abschnitten 3.3 und 3.4 wurde das Modell in bezug auf einen Teil der Parameter ausgewertet. Eine Zusammenfassung der Ergebnisse und die entsprechenden 
Tabelle 3.1: Ergebnisse bei endogener Sparentscheidung

\begin{tabular}{c|ccccc} 
& $C^{*}$ & $H^{*}$ & $t H^{*}$ & $D^{*}$ & $\mathrm{~A}$ \\
\hline$p$ & $\gtreqless 0, \gtreqless 0$ & $<0, \gtreqless 0$ & $<0, \gtreqless 0$ & $>0,=0$ & $\gtreqless 0, \gtreqless 0$ \\
$\alpha$ & $>0, \gtreqless 0$ & $<0, \gtreqless 0$ & $<0, \gtreqless 0$ & $\gtreqless 0,=0$ & $\gtreqless 0, \gtreqless 0$ \\
$t$ & $\gtreqless 0, \gtreqless 0$ & $<0, \gtreqless 0$ & $<0, \gtreqless 0$ & $\gtreqless 0,=0$ & $\gtreqless 0, \gtreqless 0$ \\
$r$ & $\gtreqless 0, \gtreqless 0$ & $>0,>0$ & $>0,>0$ & $\gtreqless 0,=0$ & $\gtreqless 0,>0$ \\
$w_{1}$ & $>0,>0$ & $>0,>0$ & $>0,>0$ & $\gtreqless 0,=0$ & $\gtreqless 0,>0$ \\
$w_{2}$ & $>0,>0$ & $>0,>0$ & $>0,>0$ & $\gtreqless 0,=0$ & $\gtreqless 0,<0$
\end{tabular}

Resultate für die übrigen Parameter enthält Tabelle 3.1. Ihre Felder geben zwei Vorzeichen an: Das linke bezieht sich auf die innere Lösung, das rechte auf die Ecklösung. Die Ergebnisse für die innere Lösung in den vier letzten Zeilen beruhen auf der Annahme einer abnehmenden absoluten Risikoaversion. In bezug auf $p$ und $\alpha$ braucht man diese Annahme nicht oder sie nützt nichts. Für die Ecklösung gilt dies generell.

Die dritte Zeile für die innere Lösung soll kurz kommentiert werden. Der Einfluß der Besteuerung auf die Verkürzung von Steuern widerspricht hier wieder den gängigen Vorstellungen über diesen Zusammenhang (siehe Anhang 3.5). Das Ergebnis $\partial H^{*} / \partial t<0$ beruht jedoch auf denselben Annahmen - insbesondere einer abnehmenden absoluten Risikoaversion -, die man benötigt, um die plausible Vermutung zu bestätigen, daß der Steuerbetrug durch ein höheres (exogenes) Einkommen stimuliert wird. In dem Modell kürzt eine höhere Steuer die exogenen Einkommen in den Perioden 1 und 2. Zusätzlich senkt sie die Verzinsung der Ersparnisse nach Steuer. Insofern entspricht die Erhöhung von $t$ einem sinkenden Bruttozins $r$. Insgesamt ist es daher nicht verwunderlich, daß ein Stpfl. mit einer abnehmenden absoluten Risikoaversion geringere Zinserträge hinterzieht, wenn die proportionale Steuer erhöht, die Kontrollen und Strafen gleichzeitig aber nicht gelockert werden.

Vergleichen wir die Ergebnisse für den Fall einer inneren Lösung insgesamt mit den entsprechenden Resultaten des Grundmodells. Die Aussagen über das hinterzogene Einkommen und die hinterzogene Steuer bleiben erhalten, wenn man die Annahme eines exogenen Zinseinkommens aufgibt. Da das Zinseinkommen $r\left(l_{1}-C^{*}\right)$ jedoch auf Parameteränderungen reagiert, diese Reaktionen im allgemeinen aber nicht vorhersagbar sind, ist es kaum möglich, eindeutiges über die Entwicklung des deklarierten Einkommens $D^{*}=r\left(l_{1}-C^{*}\right)-H^{*}$ und des erwarteten Aufkommens aus der Besteuerung von Zinserträgen zu sagen. Es ergibt sich hier 
wie in Pencavel (1979) das Ergebnis, daß bei endogenem Einkommen die Entwicklung des hinterzogenen besser als die des deklarierten Einkommens vorhersagbar ist. Insgesamt gesehen kann man festhalten, daß die Erweiterung um ein endogenes Kapitaleinkommen die aus dem Grundmodell bekannten Zusammenhänge in Grenzen bestätigt. Für das Modell mit endogenem Arbeitseinkommen von Pencavel (1979) kann man das dagegen nicht behaupten - obwohl er auch mit einer (in Konsum und Arbeitszeit) additiv separablen Nutzenfunktion arbeitet (siehe Pencavel, 1979, S. 119, 116, und Abschnitt 2.2).

Die innere Lösung ist nur einer von zwei möglichen Fällen. Das Verhalten eines Steuerhinterziehers, der keine Zinserträge angibt, ist jedoch im Gegensatz zu dem ersten Fall nicht vorhersagbar, wenn finanzpolizische Parameter geändert werden. Nur die Änderung des deklarierten Einkommens ist bei der Ecklösung laut Annahme null.

Bisher wurde mit einem proportionalen Tarif und der Strafe $\Phi_{1}$ gearbeitet. Die zwei letzten Abschnitte dieses Kapitels geben jeweils eine dieser vereinfachenden Annahmen auf.

\subsection{Die Strafe nach deutschem Recht}

Die Strafe nach deutschem Recht hängt von dem (tatsächlichen) Nettoeinkommen und der hinterzogenen Steuer ab (siehe Abschnitt 2.1.1). Das Nettoeinkommen des Sparers in Periode 2 beträgt $(1-t)\left(w_{2}+r\left(l_{1}-C\right)\right)$. Die einfachste Version der Strafe nach deutschem Recht ist

$$
\Phi_{3}=\left[\gamma(1-t)\left(w_{2}+r\left(l_{1}-C\right)\right)\right] t H
$$

Bei endogenem Zinseinkommen ist die Größe in der eckigen Klammer, mit der die hinterzogene Steuer multipliziert wird, von der Ersparnis $l_{1}-C$ der letzten Periode abhängig. Sobald $C$ sich also ändert, variiert auch dieser Multiplikator. Diese Eigenschaft der Straffunktion hat Folgen für das Verhalten des Stpfl.

Das Optimierungsproblem

$$
\begin{array}{cl}
\max _{C, H} & V(C)+q W\left(l_{2}+R\left(l_{1}-C\right)+t H\right) \\
& +p W\left(l_{2}+R\left(l_{1}-C\right)-\gamma(1-t)\left(w_{2}+r\left(l_{1}-C\right)\right) t H\right) \\
\text { u.d.N. } & 0 \leqq H \leqq r\left(l_{1}-C\right)
\end{array}
$$

liefert für eine innere Lösung bezüglich $C$ und $H$ die notwendigen Bedingungen

$$
\begin{aligned}
& V^{\prime}\left(C^{*}\right)-q W^{\prime}(G) R-p W^{\prime}(P)\left(R-\gamma(1-t) r t H^{*}\right)=0 \\
& q W^{\prime}(G)-p W^{\prime}(P) \gamma(1-t)\left(w_{2}+r\left(l_{1}-C^{*}\right)\right)=0
\end{aligned}
$$


Die deutsche Strafe modifiziert die Bedingung für eine optimale intertemporale Allokation des Konsums: Mit $C$ sinkt die Strafe für den Steuerbetrug um $\gamma(1-t) r t H$ pro Konsumeinheit. Der zukünftige Konsum $P$ vermindert sich um weniger als $R=1+(1-t) r$, wenn der gegenwärtige Konsum um eine Einheit steigt. Das intertemporale Preisverhältnis zwischen $C_{1}$ und $C_{2}$ ist damit von dem hinterzogenen Einkommen abhängig. Auf der anderen Seite ist der Preis der Hinterziehung von Steuern, $\gamma(1-t)\left(w_{2}+r\left(l_{1}-C\right)\right)$, den man (3.10) entnehmen kann, von dem Konsum in Periode 1 abhängig, wenn die Geldstrafe mit dem Einkommen des Stpfl. steigt.

Da die zwei relativen Preise, mit denen der Haushalt rechnet, von jeder Änderung der Parameter des Modells beeinflußt werden - direkt und indirekt über die induzierte Änderung von $C^{*}$ und $H^{*}$-, ist es nicht verwunderlich, daß eindeutige komparativ-statische Ergebnisse nicht zu erhalten sind ${ }^{3}$. Bezüglich der Parameter $w_{i}, r$ und $t$ ist kein anderes Ergebnis zu erwarten, da sie schon bei einem exogenen Einkommen in Periode 2 (gegebenem $C$ ) keine Vorhersage über das hinterzogene Einkommen zulassen. Mit endogenen Zinserträgen ist jedoch zusätzlich die Wirkung verschärfter Kontrollen und eines höheren Strafsatzes $\gamma$ nicht prognostizierbar. Sie können auch zu einem höheren hinterzogenen Einkommen führen. Das liegt hier jedoch nicht etwa daran, daß der nicht gesetzestreue Haushalt nicht auf Instrumente der Rechtsdurchsetzung reagiert, weil andere als ökonomische Motive sein Verhalten steuern - ein häufiger Einwand gegen eine Verschärfung von Strafen und Kontrollen. Die Ergebnisse sind im Gegenteil darauf zurückzuführen, daß der Stpfl. die Änderung der relativen Preise seines Optimierungsmodells, die mit einer intensiveren Verfolgung des Steuerbetruges verbunden ist, beachtet.

\subsection{Direkt progressiver Tarif}

In diesem Abschnitt wird überprüft, ob sich einige der in Abschnitt 3.5 zusammengefaßten Ergebnisse für den direkt progressiven Tarif $T(B)$ auf der Bemessungsgrundlage $B>0$ mit den Eigenschaften

$$
\begin{aligned}
& 0<T(B)<B \\
& 0<T^{\prime}(B)<1 \\
& 0<T^{\prime \prime}(B)
\end{aligned}
$$

verallgemeinern lassen. Der Abschnitt beschränkt sich auf die Untersuchung des optimalen Umfangs der hinterzogenen Steuer, die statt des hinterzogenen Einkommens als Entscheidungsvariable des Haushalts verwendet wird. Diese Substitution

\footnotetext{
${ }^{3} \mathrm{Es}$ wird darauf verzichtet, die unbestimmten Resultate zu dokumentieren.
} 
ist durch Abschnitt 2.1.3 motiviert, der gezeigt hat, daß bei einer direkten Progression die Änderung der hinterzogenen Steuer besser vorhersagbar ist als die des hinterzogenen Einkommens. Da die hinterzogene Steuer die zentrale Größe in der Auseinandersetzung zwischen dem Stpfl. und der Finanzbehörde ist, wird der ökonomisch interessante Kern des Modells durch diese Substitution nicht berührt.

Sei $N(B)=B-T(B)$ die Residualeinkommensfunktion: Versteuert der Haushalt ein Einkommen $B$, bleibt ihm davon $N(B)$. Diese Funktion vereinfacht die Darstellung. Ihre Eigenschaften sind

$$
\begin{aligned}
& B>N(B)>0 \\
& 1>N^{\prime}(B)>0 \\
& 0>N^{\prime \prime}(B)
\end{aligned}
$$

Wird der Stpfl. nicht geprüft, ist sein Konsum in Periode 2

$$
\begin{aligned}
G= & w_{2}+(1+r)\left(N\left(w_{1}\right)-C\right)-T\left(w_{2}+r\left(N\left(w_{1}\right)-C\right)\right) \\
& +\left[T\left(w_{2}+r\left(N\left(w_{1}\right)-C\right)\right)-T\left(w_{2}+r\left(N\left(w_{1}\right)-C\right)-H\right)\right] \\
= & N\left(w_{1}\right)-C+N\left(w_{2}+r\left(N\left(w_{1}\right)-C\right)\right)+S
\end{aligned}
$$

Die eckige Klammer enthält die hinterzogene Steuer $S$. Gleichung (3.11) besagt, daß der Stpfl. seine Ersparnisse $N\left(w_{1}\right)-C$, das tatsächliche Einkommen nach Steuer und die hinterzogene Steuer für den Konsum verwenden kann, falls er einer Prüfung entgeht. Im anderen Fall ist der Konsum in Periode 2 gleich

$$
P=N\left(w_{1}\right)-C+N\left(w_{2}+r\left(N\left(w_{1}\right)-C\right)\right)-\alpha S
$$

wobei $\alpha>0$ der konstante Strafsatz ist. Das Problem des Haushalts ist

$$
\begin{array}{cl}
\max _{C, S} & V(C)+q W(G)+p W(P) \\
\text { u.d.N. } & 0 \leqq S \leqq T\left(w_{2}+r\left(N\left(w_{1}\right)-C\right)\right)-T\left(w_{2}\right)
\end{array}
$$

wobei $G$ und $P$ aus (3.11) und (3.12) eingesetzt werden. Die rechte Schranke für $S$ ist die maximal hinterziehbare Steuerschuld. Sie ist gleich der Differenz zwischen der Steuer auf das tatsächliche Einkommen und der Steuer, die sich ergibt, wenn keine Zinsen deklariert werden. Den Betrag $T\left(w_{i}\right)$ kann der Haushalt nicht verkürzen, da die Löhne einer Lohnsteuer mit dem Tarif $T(B)$ unterliegen.

Die Bedingungen erster Ordnung für eine innere Lösung sind

$$
\begin{gathered}
V^{\prime}\left(C^{*}\right)-\left(1+N^{\prime}\left(w_{2}+r\left(N\left(w_{1}\right)-C^{*}\right)\right) r\right)\left(q W^{\prime}(G)+p W^{\prime}(P)\right)=0 \\
q W^{\prime}(G)-p W^{\prime}(P) \alpha=0
\end{gathered}
$$


Die hinterzogene Steuer $S^{*}=S^{*}\left(p, \alpha, r, w_{1}, w_{2}\right)$ ist von fünf Parametern abhängig. Eine Intensivierung der Kontrollen und die Verschärfung der Strafen zeitigen hier wieder das erwartete Verhalten: $\partial S^{*} / \partial p, \partial S^{*} / \partial \alpha<0$ (Anhang 3.7). Der Effekt eines höheren Zinssatzes ist

$$
\begin{aligned}
\frac{\partial S^{*}}{\partial r}= & \Delta^{-1}\left(R_{a}(G)-R_{a}(P)\right) q W^{\prime}(G)\left[V^{\prime \prime}\left(C^{*}\right) N^{\prime}\left(N\left(w_{1}\right)-C^{*}\right)\right. \\
& -\left(q W^{\prime}(G)+p W^{\prime}(P)\left\{N^{\prime \prime}\left(N\left(w_{1}\right)-C^{*}\right) r+N^{\prime}+N^{\prime 2} r\right\}\right]
\end{aligned}
$$

Die Ableitungen der Residualeinkommensfunktion sind mit $N^{\prime}$ und $N^{\prime \prime}$ abgekürzt, da sie stets an derselben Stelle $w_{2}+r\left(N\left(w_{1}\right)-C^{*}\right)$ bewertet werden. Eine hinreichende Bedingung für $\partial S^{*} / \partial r>0$ ist ein positives Vorzeichen des Terms in der geschweiften Klammer. $N^{\prime \prime}$ ist jedoch infolge der direkten Progression negativ. Man muß daher Annahmen über das Verhältnis von $N^{\prime}$ und $N^{\prime \prime}$ treffen. Gehen wir beispielshalber von einem Tarif mit konstanter Residualeinkommenselastizität $\rho=N^{\prime}(B) B / N(B)$ aus, der sich in der steuertheoretischen Literatur einer gewissen Prominenz erfreut (siehe Jakobsson, 1976). Bei einer Änderung von $B$ bleibt $\rho$ konstant, wenn

$$
N^{\prime \prime}(B) B+N^{\prime}(B)-N^{\prime}(B)^{2} B=0
$$

gilt. Da in unserem Fall $B=w_{2}+r\left(N\left(w_{1}\right)-C^{*}\right)$ ist, hat die geschweifte Klammer ein positives Vorzeichen. Eine konstante Residualeinkommenselastizität wäre damit eine (mehr als) hinreichende Bedingung für $\partial S^{*} / \partial r>0$.

Der Ersatz des proportionalen durch einen progressiven Tarif führt nicht zu wesentlich anderen Ergebnissen. Diese Eigenschaft des Modells mit endogenem Zinseinkommen war schon bei den Erweiterungen des Grundmodells in Kapitel 2 aufgefallen.

\subsection{Zusammenfassung}

Dieses Kapitel hat untersucht, in wieweit sich die aus dem Partialmodell mit exogenem Einkommen bekannten Zusammenhänge verallgemeinern lassen, wenn man ein endogenes Zinseinkommen, eine in dem Konsum verschiedener Perioden additiv separable Nutzenfunktion und eine abnehmende absolute Risikoaversion annimmt. Für einen Steuerpflichtigen, der nur einen Teil seiner Zinserträge hinterzieht, ist eine Verallgemeinerung zum Teil möglich. Sie stößt jedoch an zwei Punkten an Grenzen: (i) Die Reaktion des deklarierten Einkommens auf Parameteränderungen ist - im Gegensatz zu dem hinterzogenen Einkommen - auch mit einer einfachen Straffunktion kaum vorhersagbar. Dies hat zur Konsequenz, daß auch die Entwicklung des Steueraufkommens unbestimmt bleibt. Eine Erhöhung der Entdeckungswahrscheinlichkeit etwa senkt zwar das hinterzogene Einkommen, ist aber 
nicht unbedingt mit einem höheren Aufkommen aus der Besteuerung von Zinserträgen verbunden, da die Sparbereitschaft möglicherweise sinkt. (ii) Die Strafe nach deutschem Recht, die von der hinterzogenen Steuer und dem Nettoeinkommen des Steuerhinterziehers abhängt, läßt nicht einmal bei einer Änderung von Strafsatz und Entdeckungswahrscheinlichkeit eine Prognose zu.

Mit einem endogenen Zinseinkommen wird auch die Analyse eines Steuerpflichtigen interessant, der seine gesamten Zinserträge hinterzieht. Die Ecklösung führt jedoch auch bei proportionalem Tarif und einer einfachen Straffunktion in aller Regel nicht zu eindeutigen Ergebnissen. Es ist daher wichtig zu wissen, ob "der repräsentative Steuerhinterzieher" alle oder nur einen Teil seiner Zinserträge hinterzieht, wenn man über Wege zur Erfassung dieser Einkommen spricht.

Am Schluß dieses Kapitels soll ein schwacher Punkt des dargestellten Modells angesprochen werden: Es geht von einer einzigen Anlageform der Ersparnisse aus. Im Zusammenhang mit der Hinterziehung von Kapitalerträgen ist jedoch die Unterscheidung von In- und Auslandsanlagen bedeutsam. Die Wahrscheinlichkeit, daß eine Hinterziehung der Zinsen entdeckt wird, dürfte bei Auslandsanlagen oft geringer als bei Anlagen im Inland sein, da der Hoheitsbereich des Fiskus an der Grenze endet und von einer reibungslosen Zusammenarbeit der Steuerfahndung mit den entsprechenden ausländischen Stellen zumeist nicht ausgegangen werden kann ${ }^{4}$. Wenn ein Sparer Auslandsanlagen in Betracht zieht, die mit einem Wechselkursrisiko verbunden sind, wäre das Modell dieses Kapitels zu erweitern: Eine zusätzliche, riskante Anlagemöglichkeit und die Hinterziehung der entsprechenden Erträge kämen dazu.

Neben Anlagen in ausländischer Währung gibt es jedoch auch DM-Auslandsanleihen. Das Wechselkursrisiko entfällt hier, so daß als wesentlicher Unterschied zu einer Inlandsanlage eine eventuell geringere Entdeckungswahrscheinlichkeit übrigbleibt, sofern die Auslandsanlagen keine Spuren auf Depotauszügen von Banken im Inland hinterlassen. Wenn die Senkung der Entdeckungswahrscheinlichkeit durch die Anlage im Ausland groß genug ist, um etwaige Zinsdifferenzen und andere zusätzliche Kosten aufzuwiegen, wird ein Stpfl., der nur an dem Erwartungsnutzen seines Nettoeinkommens interessiert ist, seine gesamten Ersparnisse in DM-Auslandsanlagen investieren. Damit wäre man im Ergebnis wieder bei einem Modell mit einer Anlageform angelangt.

Es soll hier nicht weiter ein Modell strapaziert werden, mit dem auf möglichst einfache Weise die Steuerhinterziehung bei einer endogenen Sparentscheidung analysiert werden sollte. Neben der Berücksichtigung von Auslandsanlagen könnte man sich viele andere Erweiterungen vorstellen. Interessant wäre z.B. festzustel-

\footnotetext{
${ }^{4}$ Das gilt nicht für jedes Land. Die amerikanischen Finanzbehörden leiten von sich aus die Kontrollmitteilungen der Banken über Kapitalerträge von Ausländern an die entsprechenden ausländischen Behörden weiter (vgl. Bielsdorfer, 1989, S. 95).
} 
len, welchen Einfluß ein Vererbungsmotiv des Sparers auf die Hinterziehung der Zinsen hat. Bei der Vererbung angesparten Vermögens entsteht für die Erben nämlich folgender Vorteil: Wenn die Hinterziehung der Zinsen erst nach dem Tode des Erblassers entdeckt wird - aufgrund der Mitteilungen der Banken und der Gerichte an die Erbschaftsteuerstelle, die nach der Untersuchung des Bundesrechnungshofes (1985) nur zu $33 \%$ an die für die Einkommensteuer des Erblassers zuständige Veranlagungsdienststelle weitergegeben werden -, müssen die Erben zwar die hinterzogene Einkommensteuer nachzahlen, nicht aber eine Strafe für die Steuervergehen des Erblassers aufbringen. In dem nächsten Kapitel soll es jedoch nicht um diese, sondern um eine andere Erweiterung gehen: die Quellensteuer auf Zinserträge. 


\section{Kapitel 4}

\section{Die Quellensteuer auf Zinserträge}

\section{und die Hinterziehung von Einkommensteuern}

\subsection{Einleitung}

Beginnen wir mit einer kurzen Chronik. Im Oktober 1987 wurde für 1989 eine Quellensteuer von $10 \%$ auf Zinsen aus festverzinslichen Forderungen angekündigt. Diese Maßnahme war überraschend, da sie mit Hinweis auf die grundsätzliche Steuerehrlichkeit der Sparer zuvor abgelehnt worden war (siehe Bundesministerium der Finanzen, 1986, S. 12). Der Grund für die neue Steuer war die Finanzierungslücke, die als Folge der Entlastungen durch die "Steuerreform 1990" erwartet wurden. Über 4 Mrd. DM sollte die Quellensteuer einbringen. Als Begründung wurde die insbesondere im Vergleich zu den Lohneinkommen ungleichmäßige Versteuerung der Zinserträge angeführt. Vorsichtshalber wurde die Quellensteuer mit verschiedenen Konzessionen an die Anleger versehen: Neben den bescheidenen Satz von $10 \%$ traten Ausnahmeregelungen für bestimmte Sparformen und Sparer, die Übernahme des Bankenerlasses in die Abgabenordnung und ein Verzicht auf Steuernachforderungen bei der Nacherklärung von Kapitalerträgen.

Schon bevor die Quellensteuer Anfang 1989 in Kraft trat, zeigte sie Wirkungen - obwohl sich für einen Steuerzahler, der seine Einkünfte in vollem Umfang dem Finanzamt deklariert, durch ihre Erhebung nichts ändert; ihm werden die von den Banken an das Finanzamt abgeführten $10 \%$ der Zinsen auf seine Einkommensteuerschuld angerechnet ${ }^{1}$. Viele (kleine) Sparer schichteten ihr Vermögen in quellen-

\footnotetext{
${ }^{1}$ Das ist nicht allen Steuerzahlern klargeworden. Eigenen Umfragen zufolge dachten einige,
} 
steuerfreie inländische Anlagen um (Sparkonten mit gesetzlicher Kündigungsfrist). Andere Anleger exportierten ihr Kapital. Der beträchtliche Kapitalexport in 1988 kann allerdings nicht ausschließlich auf die Quellensteuer zurückgeführt werden (vgl. Krischausky, 1988, S. 147 - 155) - zumal deshalb, weil viele ausländische Staaten eine Quellensteuer mit wesentlich höheren Sätzen als 10 \% erheben oder Kontrollmitteilungen schreiben (für eine Übersicht siehe z.B. Rürup, 1989). Im Frühjahr 1989 kam es zu einem Kabinetts-Revirement. Der neue Finanzminister schaffte die Quellensteuer ersatzlos ab.

Liest man die zustimmende Bewertung dieser Entscheidung durch einen prominenten Vertreter der Banken (Geiger, 1989), kann man sich des Eindrucks nicht erwehren, daß die Quellensteuer die in sie gesetzten Erwartungen hätte erfüllen können, wenn sie nicht durch Ausnahmeregelungen und vor allem durch den Kapitalexport unterlaufen worden wäre. Der Frage, ob dieser Eindruck richtig ist, geht dieses Kapitel nach. Immerhin ist nicht auszuschließen, daß die Quellensteuer auf Kapitalerträge in Zukunft unter idealeren Bedingungen - insbesondere einer engeren Abstimmung der Steuerpolitik an den wichtigsten Finanzplätzen der Europäischen Gemeinschaft - wieder eingeführt wird.

Dieses Kapitel beschäftigt sich mit den Wirkungen einer Quellensteuer auf die Zinserträge von Sparern, die nur in eine einzige sichere Geldanlage investieren können. Es versucht, die Fragen zu beantworten, ob

- die Quellensteuer zu einer geringeren Attraktivität der Hinterziehung von Zinserträgen führt und damit weniger hinterzogen wird;

- das von dem Fiskus zu erwartende Steueraufkommen durch die Erhebung der Quellensteuer steigt;

- die Quellensteuer ein Schritt zu einer gleichmäßigeren Besteuerung von Kapital- und Lohneinkommen ist.

Die Modellanalyse berücksichtigt neben der Quellensteuer auf Zinserträge die Geldstrafe nach deutschem Recht, ein endogenes Kapitaleinkommen und einen progressiven Verlauf des Einkommensteuertarifs. Sie zeigt, daß die Antwort zu den drei oben gestellten Fragen keineswegs auf der Hand liegt.

Unter einer bestimmten Bedingung verursacht die Quellensteuer sogar einen Anstieg der hinterzogenen Zinserträge und führt gleichzeitig weder zu einem höheren Aufkommen noch zu einem Mehr an Steuergerechtigkeit. Das Ergebnis, daß die Risikobereitschaft der Steuerpflichtigen mit der Quellensteuer steigt im Sinne höherer nicht deklarierter Zinserträge, ist eine Parallele zu einem Resultat aus der Literatur über "Steuern und Risikobereitschaft" (siehe Mossin, 1968, und Sandmo,

sie hätten $10 \%$ ihres Sparvermögens an den Staat abzutreten. 
1985). Dort führt eine höhere Besteuerung der Renditedifferenz unsicherer und sicherer Kapitalanlagen eines Investors zu einem Anstieg der riskanten Investitionen. Zusätzlich kann neben dem Vorzeichen auch der Betrag der Reaktion bestimmt werden. Genau dies trifft auch bei der Quellensteuer zu, wenn ein Steuerpflichtiger in der Ausgangssituation nicht seine gesamten Zinserträge hinterzieht.

Das Kapitel gliedert sich wie folgt. Abschnitt 4.2 stellt das Modell unter der Voraussetzung einer exogenen Ersparnis dar, Abschnitt 4.3 beantwortet die Frage, wie die Hinterziehung der Zinserträge von der Besteuerung an der Quelle abhängt. Abschnitt 4.4 enthält den Vergleich mit der erwähnten Parallele aus der Literatur über Steuern und Risikobereitschaft. Abschnitt 4.5 verallgemeinert die Untersuchung für den Fall endogener Zinserträge. Auf die Entwicklung des Steueraufkommens und die Frage einer gleichmäßigeren Besteuerung geht Abschnitt 4.6 ein. In Abschnitt 4.7 wird die Analyse einer proportionalen Einkommen- und Quellensteuer auf den Fall einer progressiven Einkommen- und einer proportionalen Quellensteuer übertragen.

\subsection{Das Modell}

Wir betrachten einen Haushalt mit einem Planungshorizont von einer Periode. Er hat in der Vergangenheit einen Betrag $s$ gespart und erzielt am Anfang der Periode Zinserträge in Höhe von $r s$, da nur eine Anlage zu einem sicheren Zinssatz $r$ möglich war. Da die Zinsen an der Quelle mit dem Satz $b$ besteuert werden, wird dem Haushalt von seiner Bank nur der Betrag $(1-b) r s$ gutgeschrieben. Neben den Kapitalerträgen bezieht der Haushalt noch ein Arbeitseinkommen in Höhe von $w$, das einer Lohnsteuer mit dem Satz $t$ unterliegt. Der Arbeitgeber zahlt also $w(1-t)$ an den Haushalt aus.

Kurz nach Erhalt dieser Zahlungen hat der einkommensteuerpflichtige Haushalt sein Einkommen gegenüber den Finanzbehörden zu deklarieren. Auf die dem deklarierten Einkommen entsprechende Steuerschuld wird die durch Steuerabzug erhobene Einkommensteuer (Lohn- und Quellensteuer) angerechnet. Ich nehme eine proportionale Einkommensteuer mit dem Satz $t>b$ an, so daß die Lohneinkommen gar nicht und die Zinsen teilweise hinterzogen werden können.

Nachdem die dem deklarierten Einkommen entsprechenden Steuern bezahlt worden sind, kann der Stpfl. zu einem Objekt der Steuerfahndung werden. Mit einer Wahrscheinlichkeit $q(0<q<1)$ wird der Stpfl. nicht steuerlich geprüft. Mit einer Wahrscheinlichkeit $p=1-q$ findet eine Steuerprüfung statt und führt zur Aufdeckung aller steuerlich relevanten Tatbestände. Es sind in diesem Falle - falls hinterzogen wurde - die hinterzogenen Steuern nachzuzahlen und eine Geldstrafe zu begleichen, die von der Höhe der hinterzogenen Steuern abhängt. 
Am Ende der Periode kann der Haushalt sein Einkommen nach Steuern und Strafen konsumieren. Der Konsum $C$ ist das einzige Argument seiner strikt konkaven von Neumann/Morgenstern-Nutzenfunktion $U(C)$. Die hinterzogenen Zinseinkünfte $H$ sind die Entscheidungsvariable des Stpfl. Unter Beachtung der Beschränkung $0 \leqq H \leqq r s$ wird er $H$ derart wählen, daß sein Erwartungsnutzen

$$
E(U(C))=q U\left(C_{\mid \text {keineKontrolle }}\right)+p U\left(C_{\mid \text {Kontrolle }}\right)
$$

maximal wird.

Die Budgetbeschränkungen für die beiden Zustände, in denen sich der Stpfl. wiederfinden kann, sind nun formal darzustellen. Hat der Stpfl. Glück und entgeht einer Kontrolle durch die Finanzbehörden, ist sein Konsum:

$$
C_{\mid \text {keineKontrolle }}:=G=(1+r(1-b)) s+w(1-t)-(t-b)(r s-H)
$$

Von den verzinsten Ersparnissen und dem Lohn ist die abschließende Steuerzahlung zu leisten. $r s-H$ sind die deklarierten Zinseinkünfte. Von der Steuerschuld wird die bereits von dem Schuldner der Zinsen abgeführte Quellensteuer abgezogen. Es kann allerdings nicht die gesamte Quellensteuer berücksichtigt werden, wenn $H>0$ ist. Das Finanzamt würde sich wundern, wenn ein Stpfl., der z.B. 10000 DM Zinserträge bezieht, $5000 \mathrm{DM}$ deklariert, aber (bei einem Quellensteuersatz von z.B. $10 \%$ ) 1000 DM Quellensteuern abziehen will. Nur für die deklarierten Zinserträge kann die Quellensteuer angerechnet werden.

Der Ausdruck für $\mathrm{G}$ läßt sich vereinfachen:

$$
G=(1+r(1-t)) s+l+(t-b) H
$$

wobei $l=w(1-t)$ sei. Mit jeder DM Zinseinkommen, die der Stpfl. nicht deklariert, kann er dem Fiskus $t-b$ DM vorenthalten. Je höher der Quellensteuersatz $b$ ist, desto geringer ist (bei gegebenem Einkommensteuersatz $t$ ) die mögliche Steuerersparnis. Wählt der Stpfl. $H=0$, erhalten wir die Budgetbeschränkung, die sich auch für den Fall ergibt, daß den Finanzbehörden das gesamte Einkommen vor Steuer bekannt ist. Mit $H=r s$ gilt andererseits $G_{\mid H=r s}=(1+r(1-b)) s+l$. Der Stpfl. zahlt in diesem Fall nur die Quellen- und Lohnsteuer. Die untere und obere Ecklösung bezüglich der Entscheidungsvariablen $\mathrm{H}$ wurden zur Illustration dargestellt. Der Stpfl. kann natürlich auch eine innere Lösung wählen.

Wird der Haushalt steuerlich geprüft, ändert sich (für $H>0$ ) das Bild. Die Budgetbeschränkung lautet nun:

$$
\begin{aligned}
C_{\mid \mathrm{Kontrolle}}:=P= & (1+r(1-b)) s+l-(t-b)(r s-H) \\
& -(t-b) H-\alpha(t-b) H
\end{aligned}
$$


Gegenüber dem Fall, daß der Stpfl. Glück hat, sind zwei Summanden dazugekommen. Es sind die Nachzahlung der hinterzogenen Steuerschuld und eine Geldstrafe. Die Nachzahlung beträgt nicht $t H$ sondern $(t-b) H$, da die Bank ja auch für die nicht deklarierten Zinseinkünfte bereits Einkommensteuern in Form der Quellensteuer auf Rechnung des Stpfl. vorausbezahlt hat. Dieser Umstand ist im Fall einer Kontrolle zu beachten, obwohl er - wie oben begründet wurde - nicht berücksichtigt werden kann, wenn die Hinterziehung unentdeckt bleibt.

Die Geldstrafe ist ein konstantes Vielfaches der hinterzogenen Steuer. Ich nehme im folgenden an, daß für die Gerichte $(t-b) H$ und nicht $t H$ die hinterzogene Steuer ist, sobald eine Quellensteuer erhoben wird, da Steuern auf Zinserträge nur in dem Umfang dem Zugriff des Fiskus entzogen werden können, wie sie nicht schon durch die Banken vorausgezahlt worden sind. Die Strafe

$$
\Phi_{1}=\alpha(t-b) H
$$

ist in den vorangehenden Kapiteln für den Fall ohne eine Quellensteuer, i.e. $b=0$, verwendet worden. Neben dieser Form der Bestrafung wird im folgenden die Geldstrafe nach deutschem Recht berücksichtigt. Sie ist von dem (tatsächlichen) Nettoeinkommen des Stpfl. und der hinterzogenen Steuer abhängig (siehe Abschnitt 2.1.1):

$$
\Phi_{3}=\gamma(1-t)(r s+w)(t-b) H
$$

Die Ergebnisse in der Literatur zur Steuerhinterziehung hängen im allgemeinen von der Form der Strafe ab (siehe Kapitel 2 und 3, insbesondere die Abschnitte 2.1.1, 3.5 und 3.6). Die Quellensteuer hat jedoch - soweit sei hier vorgegriffen - unter beiden oben angeführten Strafen die gleichen Wirkungen. Es wird daher im folgenden stets zuerst die Strafe $\Phi_{1}$ verwendet. Anschließend wird jeweils kurz geprüft, ob die Eigenschaft der deutschen Straffunktion, wirtschaftlich besser gestellte Stpfl. für gleiche Vergehen härter zu bestrafen als Stpfl. mit einem geringeren Nettoeinkommen, zu abweichenden Ergebnissen führen kann.

Die Budgetgleichung für den Fall, daß der Stpfl. geprüft wird, läßt sich vereinfachen:

$$
P=(1+r(1-t)) s+l-\alpha(t-b) H
$$

Mit den Budgetbeschränkungen für die beiden möglichen Zustände kann jetzt das Maximierungsproblem des Stpfl. vollständig dargestellt werden:

$$
\begin{array}{cl}
\max _{\mathbf{H}} & q U((1+r(1-t)) s+l+(t-b) H)+ \\
& p U((1+r(1-t)) s+l-\alpha(t-b) H) \\
\text { u.d.N. } & 0 \leqq H \leqq r s
\end{array}
$$

Dieses Modell wird im folgenden in bezug auf die Wirkungen der Quellensteuer ausgewertet. 


\subsection{Die Hinterziehung von Zinseinkommen}

Es wird zunächst untersucht, wie sich eine marginale Erhöhung des Satzes einer existierenden Quellensteuer auf den Umfang des hinterzogenen Zinseinkommens auswirkt. Anschließend stellt sich die Frage, welches Ergebnis sich bei der Einführung einer Quellensteuer einstellt. Von besonderem Interesse ist dabei die Einführung einer nichtmarginalen Steuer.

Die Optimalitätsbedingung erster Ordnung für eine innere Lösung des Maximierungsproblems lautet

$$
E_{H}=\left(q U^{\prime}(G)-p U^{\prime}(P) \alpha\right)(t-b)=0
$$

Sie verlangt, daß Grenznutzen und -kosten der Hinterziehung gleich sind.

Mit (4.1) können die Bedingungen für eine innere Lösung abgeleitet werden. Für $H>0$ muß $(q-p \alpha)(t-b)>0$ gelten. Wenn der erwartete Ertrag pro hinterzogener DM positiv ist, wird ein risikoscheuer Stpfl. einen positiven Betrag hinterziehen (vgl. Arrow, 1970, S. 155 f.). Von $q-p \alpha>0$ wird im folgenden ausgegangen. Man beachte, daß für gegebenes $H$ der erwartete Ertrag der Hinterziehung $(q-p \alpha)(t-b) H$ negativ von $b$ abhängt. In diesem Sinne kann man von einer verminderten Attraktivität der Hinterziehung von Zinsen aufgrund der Quellensteuer sprechen.

Die Bedingung für eine Lösung mit $H<r s$ ist

$$
(t-b)\left(q U^{\prime}(G)-p U^{\prime}(P) \alpha\right)_{\mid H=r s}<0
$$

Es kann nicht ausgeschlossen werden, daß sie nicht erfüllt ist. Die Risikoaversion des Stpfl. kann so gering und der erwartete Ertrag der Steuerhinterziehung so groß sein, daß er seine gesamten Zinseinkommen nicht deklariert. Wir müssen daher eine Fallunterscheidung treffen: Ein Stpfl. wird einen Betrag $H$ mit $0<H<r s$ hinterziehen, ein anderer $H=r s$ wählen.

Beginnen wir mit einem Haushalt, der nicht seine gesamten Zinsen hinterzieht. (4.1) bestimmt implizit die Wahl von $H$ in Abhängigkeit von den Parametern des Modells:

$$
H=H(w, r, s, q, \alpha, t, b)
$$

Von Interesse ist hier, wie die Hinterziehung auf eine marginale Erhöhung der Quellensteuer reagiert. Das Ergebnis ist

$$
\frac{\partial H}{\partial b}=\frac{H}{t-b}
$$

Um die Reaktion des Stpfl. zu prognostizieren, genügt es zu wissen, wie groß das hinterzogene Einkommen in der Ausgangssituation war und welche Werte der 
Einkommen- und Quellensteuersatz hatten. Spezifische Informationen über die Risikoeinstellung des Haushalts (z.B. eine abnehmende absolute Risikoaversion) sind nicht notwendig (vgl. Mossin, 1968, Sandmo, 1985, S. 293 - 296).

Die Interpretation dieses Resultats ist äußerst einleuchtend. Folgt der Stpfl. der Regel (4.2), ändert sich die Steuerersparnis für den Fall, daß er einer Kontrolle entgeht, nicht.

$$
\frac{\partial(t-b) H}{\partial b}=-H+(t-b) \frac{\partial H}{\partial b}=0
$$

Da die Bemessungsgrundlage der Strafe ebenfalls $(t-b) H$ ist, ändert sie sich auch nicht. Die riskante Position, die hinterzogene Steuer auf der einen und die Geldstrafe auf der anderen Seite, bleibt die gleiche. Da genau diese riskante Position in der Ausgangssituation optimal war, wird der Stpfl. sie auch nach der Erhöhung der Quellensteuer noch wählen; denn seine Budgetbeschränkung hat sich nur insoweit geändert, als die maximal mögliche Steuerersparnis $(t-b) r s$ und folglich auch die maximale Strafe mit einer höheren Quellensteuer sinken. Diese Verkürzung der Budgetbeschränkung hat aber keine Auswirkung auf die Wahl der riskanten Position, solange in der Ausgangssituation die obere Ecklösung bezüglich $\mathrm{H}$ nicht optimal war.

Dieser Zusammenhang kann graphisch dargestellt werden (vgl. Atkinson/Stiglitz, 1980, S. 105). In Abbildung 4.1 zeigt $N M$ die Funktion

$$
P=(1+r(1-t)) s+l-\alpha[G-(1+r(1-t)) s-l]
$$

die durch Eliminierung von $H$ aus den Budgetbeschränkungen für die beiden Zustände gewonnen wurde. $\mathrm{Da} d P / d G=-\alpha$ ist, handelt es sich bei dieser Funktion um eine Gerade, deren Steigung nicht von $b$ abhängt. Hinterzieht der Stpfl. eine DM mehr Steuern, steigt $G$ um 1 DM und $P$ sinkt um $\alpha$ DM. In Punkt $N$ wäre der Stpfl. ehrlich gegenüber der Finanzbehörde und könnte unabhängig davon, ob er steuerlich geprüft wird oder nicht, den gleichen Konsum realisieren $(C=(1+r(1-t)) s+l)$. Mit steigender Hinterziehung rutscht die Position des Haushalts entlang der Geraden $N M$ nach unten. In $M$ hat er die maximal mögliche riskante Position erreicht $((t-b) H=(t-b) r s)$. Der vertikale Abstand von $N$ zu $M$ mißt die maximale Strafe und der horizontale Abstand $M N$ die maximale Steuerersparnis. Durch die Anhebung der Quellensteuer von $b$ auf $b+\Delta b$ wird die Budgetgerade $N M$ um das Stück $M M^{\prime}$ gekürzt. Der Punkt, der einem bestimmten hinterzogenen Einkommen entspricht, rückt näher an $N$ heran. Die höchstmögliche riskante Position ist nun kleiner. Die maximale Steuerersparnis sinkt um $\Delta b r s$, die maximale Strafe um $\Delta b a r s$. Da aber in der Ausgangslage die innere Lösung $O$ optimal war, bleibt die Verkürzung der Budgetgeraden ohne Folgen für die riskante Position im Optimum $O$. Der Stpfl. fängt die höhere Besteuerung an der Quelle auf, indem er in größerem Umfang als zuvor Zinseinkünfte 
Abbildung 4.1: Ein Teil der Zinsen wird hinterzogen.

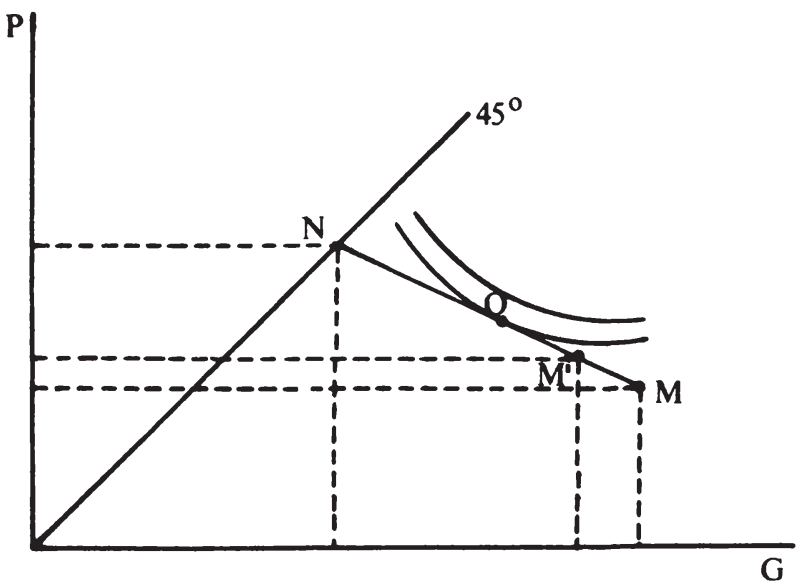

nicht deklariert.

In der Abbildung sind wir von einer nichtmarginalen Erhöhung von $b$ ausgegangen. Im Vergleich $z u$ der Ableitung $\partial H / \partial b$ sieht in diesem Fall die Reaktion des Stpfl. etwas verändert aus. Seine riskante Position bleibt gleich, wenn er $\Delta H$ aus der Gleichung

$$
(t-(b+\Delta b))(H+\Delta H)=(t-b) H
$$

bestimmt. Es ergibt sich

$$
\Delta H=\Delta b \frac{H}{t-b-\Delta b}
$$

Die diskrete Änderung $\Delta b$ kann natürlich dazu führen, daß trotz einer inneren Lösung ein Stpfl. die Quellensteuer nicht voll kompensieren kann. Es gilt dann in der Ausgangslage zwar $H<r s$, aber das zur Hinterziehung verbleibende Einkommen ist kleiner als die für eine Kompensation der Quellensteuer notwendige Reaktion: $r s-H<\Delta H$. Abbildung 4.1 ging dagegen von $r s-H>\Delta H$ aus.

Die Einführung einer Quellensteuer führt im Vergleich zu der Erhöhung einer bereits existierenden Quellensteuer nicht zu anderen Aussagen. In den beiden Reaktionsregeln für die marginale und die diskrete Reform ist lediglich der Satz $b$ der Ausgangslage gleich null zu setzen.

Die bisher abgeleiteten Ergebnisse erhält man auch mit der deutschen Straffunktion. Sie hat genau wie $\Phi_{1}$ die Basis $(t-b) H$, nur wurde der Strafsatz mit $\gamma(1-t)(r s+w)$ statt mit $\alpha$ bezeichnet. Die Unterscheidung der zwei Strafen wird 
Abbildung 4.2: Alle Zinsen werden hinterzogen.

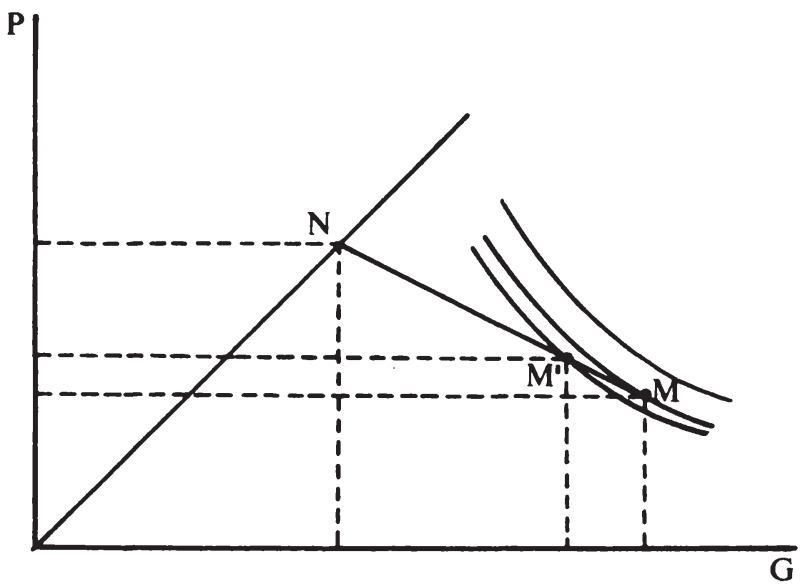

für die Analyse der Quellensteuer erst dann interessant, wenn das Einkommen modellendogen ist ${ }^{2}$. Die Strafe nach deutschem Recht beinhaltet dann nämlich im Unterschied zu $\Phi_{1}$ einen endogenen Strafsatz. Die Annahme exogener Zinseinkommen wird in Abschnitt 4.5 aufgeber.

Bisher ist von einer inneren Lösung bezüglich $H$ ausgegangen worden. Es sei nochmals betont, daß der Fall einer Wahl $H=r s$ nicht ausgeschlossen werden kann. Die Position eines Stpfl., der alle Zinserträge hinterzieht, zeigt Abbildung 4.2. Die Steigung der Indifferenzkurve in dem Optimum $M$ der Ausgangslage ist kleiner als die der Budgetgeraden $N M$. Wird die Quellensteuer erhöht, würde der Stpfl. gerne mehr Einkommen hinterziehen, um in der ursprünglichen Position $M$ zu verbleiben. Da er aber schon die obere Ecklösung $H=r s$ gewählt hat und die Zinseinkünfte exogen sind, kann er der Quellensteuer nicht ausweichen. Es gilt $\Delta H=0$ (bzw. $\partial H / \partial b=0$ ). Die hinterzogene Steuerschuld $(t-b) r s$ sinkt. Der Stpfl. landet in $M^{\prime}$ auf einem geringeren Nutzenniveau ${ }^{3}$.

\footnotetext{
${ }^{2}$ Kapitel 2 hat (für ein exogenes Einkommen) gezeigt, daß die zwei Strafen unterschiedliche Ergebnisse liefern, wenn der Einkommensteuersatz geändert wird. Nimmt man eine abnehmende absolute Risikoaversion an, steigt $H$ mit sinkendem $t$ unter $\Phi_{1}$. Die Strafe $\Phi_{3}$ läßt dagegen keine eindeutige Aussage zu.

${ }^{3}$ Tillmann (1990) untersucht für den Fall einer für alle Haushalte gleichen Nutzenfunktion, wie sich die Stpfl. bei verschiedenen Annahmen über die Verteilung der (exogenen) Lohnund Zinseinkommen in der Bevölkerung auf die zwei Gruppen der Teil- und Totalhinterzieher von Zinsen aufteilen. Er kommt zu dem Ergebnis, daß sich die "einkommensschwachen" und
} 
Für die zwei zu unterscheidenden Gruppen von Stpfl. ist die Wirkung einer isolierten Erhöhung bzw. Einführung der Quellensteuer auf das hinterzogene Zinseinkommen bestimmt worden. Auch die Einführung einer nichtmarginalen Steuer wurde untersucht. Insbesondere die Einführung der Quellensteuer führt jedoch zu der Frage nach dem Realitätsgehalt der komparativ-statischen Übung. Muß nicht die Wahrscheinlichkeit, steuerlich geprüft zu werden, aufgrund der Besteuerung an der Quelle steigen? Kann die Entdeckungswahrscheinlichkeit $p$ als konstant vorausgesetzt werden?

Es wäre denkbar, daß den Finanzbehörden von den Banken mit der Überweisung der Quellensteuern eine Liste mit den Empfängern der entsprechenden Zinszahlungen zugehen könnte. Um eine gesetzeskonforme Besteuerung sicherzustellen, hätten die Behörden dann nur noch intern zu prüfen, ob die Anleger die Zinsen in der Einkommensteuererklärung auch deklarierten. Die Entdeckungswahrscheinlichkeit wäre gleich eins und alle Zinserträge würden angegeben werden.

Die Quellensteuer ist in der Bundesrepublik gerade deshalb eingeführt worden, um solch ein System von Kontrollmitteilungen zu vermeiden. Die ungleichmäßige Besteuerung von Lohn- und Zinseinkommen drängte unterstützt von fiskalischen Motiven auf eine Reform der Zinsbesteuerung. Den politischen Instanzen schien die Quellensteuer für diesen Zweck die weniger drastische, der Richtung nach dennoch zieladäquate Maßnahme. Die Kreditinstitute haben die Quellensteuer während eines halben Jahres pauschal, ohne Angabe der Identität der Anleger abgeführt. Diese konnten davon ausgehen, daß sich die Entdeckungswahrscheinlichkeit durch die zeitweise Erhebung der Quellensteuer nicht geändert hat.

\section{4 "The Net Taxation Case"}

Die Wirkung der Quellensteuer auf die Steuerhinterziehung hat eine Parallele in der Literatur über "Steuern und Risikobereitschaft" (Mossin, 1968, Sandmo, 1985). Es lohnt sich, dieser Parallele nachzugehen. Ich folge dabei der Darstellung von Sandmo (1985).

Ein Investor mit einem Zeithorizont von einer Periode und einem Anfangsvermögen $A$ entscheidet über dessen Aufteilung in eine risikolose Anlage $m$ und eine riskante Investition $a$. Die erste Möglichkeit offeriert einen sicheren Zins $r$, die Rendite der zweiten Anlage ist die Zufallsvariable $X$. Sie nimmt mit der Wahrscheinlichkeit $q$ den Wert $x_{1}$ und mit $p=1-q$ den Wert $x_{2}$ an. Es gelte $x_{1}>r>x_{2}$. Der Investor unterliegt einer proportionalen Steuer mit dem Satz

\footnotetext{
"einkommensstarken" Haushalte nur unter sehr speziellen Annahmen jeweils eindeutig den zwei Gruppen von Hinterziehern zuordnen lassen. Man könne daher nicht behaupten, die Quellensteuer treffe nur die "kleinen Sparer".
} 
$t$, deren Bemessungsgrundlage der "excess return" der riskanten Anlage ist: Besteuert wird der Teil der Rendite der riskanten Investition, die den sicheren Zins $r$ übersteigt. Eine negative Differenz wird subventioniert, d.h. es besteht ein voller Verlustausgleich. Die Verzinsung der Anlage $m$ bleibt steuerfrei. Sandmo bezeichnet diese Form der Besteuerung als "net taxation case" (Sandmo, 1985, S. 296). Im "gross taxation case" werden demgegenüber die Erträge aus beiden Anlagen mit dem Satz $t$ besteuert.

Das Endvermögen $Y$ des Investors kann zwei Werte annehmen, die ich mit $y_{1}$ und $y_{2}$ bezeichne. Unter Beachtung von $A=m+a$ ist es entweder

$$
\begin{aligned}
y_{1} & =(1+r) m+\left(1+x_{1}\right) a-t\left(x_{1}-r\right) a \\
& =(1+r) A+(1-t)\left(x_{1}-r\right) a
\end{aligned}
$$

oder

$$
y_{2}=(1+r) A+(1-t)\left(x_{2}-r\right) a
$$

Der Vergleich dieser beiden Budgetbeschränkungen mit $G$ und $P$ aus Abschnitt 4.2 zeigt die Parallele: Während die Besteuerung des excess returns die Nettorendite der riskanten Anlage kürzt, verringert die Quellensteuer den Ertrag und die Bestrafung der Hinterziehung eines bestimmten Einkommens.

Maximiert der Investor den Erwartungsnutzen $E(U(Y))$ durch geeignete Wahl von a, ergibt sich die notwendige Bedingung

$$
q U^{\prime}\left(y_{1}\right)\left(x_{1}-r\right)+p U^{\prime}\left(y_{2}\right)\left(x_{2}-r\right)=0
$$

Die Frage ist, wie die unsichere Anlage $a$ auf eine Erhöhung der Steuer auf die Reingewinne reagiert. Die Differentiation der Bedingung erster Ordnung ergibt

$$
\frac{\partial a}{\partial t}=\frac{a}{1-t}
$$

Die riskante Position des Investors vor Steuer steigt. Seine riskante Position nach Steuer $(1-t)(X-r) a$ ändert sich jedoch nicht, wie folgende Rechnung zeigt:

$$
\frac{\partial(X-r)(1-t) a}{\partial t}=(X-r)\left[-a+(1-t) \frac{\partial a}{\partial t}\right]=0
$$

Für jede Realisation der unsicheren Rendite $X$ bleibt der private Ertrag der Investition $a$ von der höheren Steuerbelastung unberührt. "Social risk taking" $a$, d.h. die unsichere Anlage des Investors und des Fiskus zusammen, steigt mit $t$, während die private Risikobereitschaft, gemessen an $(1-t) a$, konstant bleibt (Atkinson/Stiglitz, 1980, S. 105).

$\mathrm{Zu}$ einem quantitativ vorhersagbaren Ergebnis führt nur die Besteuerung des exess returns. Die Besteuerung des Kapitaleinkommens $x_{i} a+r m$ hat dagegen neben dem oben angegebenen Substitutions- einen Einkommenseffekt zur Folge. Der 
Nettoeffekt kann mit speziellen Annahmen über die Nutzenfunktion gegebenfallas qualitativ, nicht aber quantitativ vorhergesagt werden (vgl. Sandmo, 1985, S. 295 - 97).

Sowohl die Besteuerung des excess returns als auch die Quellensteuer führen lediglich zu einem Substitutionseffekt: Bei unverändertem Erwartungsnutzen steigt die riskante Anlage bzw. das hinterzogene Einkommen. Da keine Kompensation (kein Transfer) notwendig ist, um den Stpfl. auf demselben Nutzenniveau zu halten, ist der Einkommenseffekt null (vgl. Mossin, 1968, S. 76 f., Sandmo, 1985, S. 296). Neben dieser Gemeinsamkeit bleibt ein Unterschied bezüglich der Relevanz der zwei Steuern. Die ausschließliche Besteuerung der überschüssigen Gewinne kommentiert Sandmo mit den Worten: "This case may not be entirely unrealistic" (Sandmo, 1969, S. 301). Es fällt allerdings schwer, diese Möglichkeit in der steuerlichen Realität wiederzufinden. Die Bemessungsgrundlage der existierenden Einkommensteuersysteme ist doch etwas breiter als die Renditedifferenz unsicherer und sicherer Kapitalanlagen der Steuerzahler. Sandmo dachte an den speziellen Fall, daß ein Stpfl. die riskante Anlage durch einen Kredit finanziert und Schuldzinsen bei der Einkommensteuer abziehbar sind (Sandmo, 1969, S. 597). Mit $A=0$ bzw. $a=-m$ führt die Besteuerung des (Kapital-)Einkommens (mit Abziehbarkeit von Schuldzinsen) in der Tat zu der gleichen Budgetbeschränkung wie die Besteuerung des excess returns. Das Modell wäre mit $A=0$ jedoch nicht mehr plausibel, da der Investor mit der Realisation $X=x_{2}$ ein negatives Endvermögen hätte und der Kreditgeber folglich nicht den versprochenen "sicheren Zins" $r$ verdiente. Die beiden von Sandmo betrachteten Formen der Besteuerung führen nur mit $r=0 \mathrm{zu}$ demselben Ergebnis.

Die Quellensteuer auf Zinserträge hat damit einem Zusammenhang aus der steuertheoretischen Grundlagenforschung zumindest für kurze Zeit eine Anwendung gebracht, wenn es Stpfl. gibt, die nicht ihre gesamten Zinserträge hinterzogen haben.

\subsection{Variable Zinserträge}

Bisher wurde eine gegebene Ersparnis vorausgesetzt. Diese Annahme wird jetzt aufgegeben: Der Stpfl. bestimmt durch seine Ersparnis die Höhe seines Zinseinkommens und entscheidet über den Umfang der Hinterziehung aus diesem Einkommen. Diese Erweiterung des Modells hat eine einfache Begründung. Zwischen der Steuerbelastung bzw. den Möglichkeiten, der Besteuerung zu entgehen, und der Entstehung des Einkommens gibt es im allgemeinen einen Zusammenhang. Ein Haushalt wird bei seiner Sparentscheidung die zu erwartende Steuerbelastung der Erträge berücksichtigen. Es fragt sich, wie sich dieser Zusammenhang auf die 
Effekte der Quellensteuer auswirkt. Es wird zunächst die Reaktion der Ersparnis und des hinterzogenen Zinseinkommens auf eine Erhöhung der Quellensteuer bestimmt. Der nächste Abschnitt wird dann das zu erwartende Aufkommen der Quellensteuer in dem Modell mit zwei Entscheidungsvariablen thematisieren.

Der Stpfl. lebt zwei Perioden. In jeder Periode verdient er ein exogenes Lohneinkommen von $w_{1}$ bzw. $w_{2}$. Nach Abzug der Lohnsteuer erhält er $w_{1}(1-t)=l_{1}$ bzw. entsprechend $l_{2}$ ausgezahlt. In Periode 1 entscheidet er über die Höhe seines Konsums $C_{1}$ in dieser Periode. Die Ersparnis $l_{1}-C_{1}$ ist damit endogen. Am Anfang der Periode 2 erzielt der Stpfl. Zinserträge nach Abzug der Quellensteuer in Höhe von $r(1-b)\left(l_{1}-C_{1}\right)$. Er muß dann sein Einkommen deklarieren und wird im Anschluß entweder steuerlich geprüft oder entgeht der Steuerfahndung. Zum Schluß seines Lebens kann er das Einkommen nach Steuer und Strafe der Periode 2 konsumieren. Ich gehe zunächst wieder von der Strafe $\Phi_{1}$ aus. Der Konsum des Stpfl. ist entweder

$$
C_{2 \mid \text { keineKontrolle }}:=G=(1+r(1-t))\left(l_{1}-C_{1}\right)+l_{2}+(t-b) H
$$

oder in dem weniger glücklichen Zustand

$$
C_{2 \mid \text { Kontrolle }}:=P=(1+r(1-t))\left(l_{1}-C_{1}\right)+l_{2}-\alpha(t-b) H
$$

Der Stpfl. hat eine strikt konkave Nutzenfunktion $U\left(C_{1}, C_{2}\right)$. Kürzt man $C_{1}$ mit $C$ ab, ist seine Optimierungsaufgabe

$$
\max _{\mathrm{C}, \mathrm{H}, \lambda} L=q U(C, G)+p U(C, P)+\lambda\left(r\left(l_{1}-C\right)-H\right)
$$

wobei $\lambda$ der Lagrange-Multiplikator der Nebenbedingung ist.

Es wird angenommen, daß der Haushalt positive Beträge spart. Ein Stpfl., der sich verschuldet, hätte keine Gelegenheit, Zinserträge zu hinterziehen, und ist daher für die Analyse der Quellensteuer nicht von Interesse. Zweitens kann in einem intertemporalen Modell von einem positiven Konsum in jeder Periode ausgegangen werden, so daß Ecklösungen bezüglich $C$ nicht zu befürchten sind.

Die Bedingungen erster Ordnung sind (vgl. Nikaido, 1968, S. 51 - 53)

$$
\begin{aligned}
& q U_{1}(C, G)+p U_{1}(C, P) \\
& -(1+r(1-t))\left(q U_{2}(C, G)+p U_{2}(C, P)\right)-\lambda r=0 \\
& q U_{2}(C, G)(t-b)-p U_{2}(C, P)(t-b) \alpha-\lambda=0 \\
& \lambda\left(r\left(l_{1}-C\right)-H\right)=0 \\
& \lambda \geqq 0
\end{aligned}
$$

$U_{x}(\cdot, \cdot)$ bezeichnet die erste Ableitung nach dem $x$-ten Argument der Nutzenfunktion. (4.3) ist die Bedingung für eine optimale Allokation der Ressourcen des Haushalts über die Zeit. (4.4) bestimmt die Hinterziehung. 
Die Sparer sind hier wieder in zwei Klassen zu unterteilen. Die einen deklarieren keine Zinseinkommen, die anderen hinterziehen sie nur zum Teil. In dem ersten Fall gilt $\lambda>0$, im zweiten Fall $\lambda=0^{4}$. Die Analyse beginnt mit einem Mitglied der zweiten Klasse.

Die implizite Differentiation des Gleichungssystems (4.3) und (4.4) mit $\lambda=0$ ergibt

$$
\frac{\partial H}{\partial b}=\frac{H}{t-b}
$$

und

$$
\frac{\partial C}{\partial b}=0
$$

Der Stpfl. verändert auch in dem Modell mit einer variablen Ersparnis seine riskante Position gegenüber den Finanzbehörden trotz einer höheren Quellensteuer nicht. Er hinterzieht gerade soviel mehr Einkommen, daß die hinterzogenen Steuern konstant bleiben. Kann der Haushalt durch eine höhere Hinterziehung von Zinsen dieselbe Wahrscheinlichkeitsverteilung des Konsums in der zweiten Periode erreichen wie in der Ausgangssituation, besteht kein Grund, warum er seine Sparentscheidung revidieren sollte (vgl. Sandmo, 1985, S. 300, 1969, S. 597). Die Quellensteuer hat keine Auswirkung auf die Höhe der Zinseinkommen, da sie voll kompensiert wird.

Für eine diskrete Änderung von $b$ und die Einführung einer Quellensteuer gelten entsprechende Ergebnisse. Die deutsche Straffunktion führt nicht zu anderen Reaktionen als $\Phi_{1}$, solange eine innere Lösung vorausgesetzt werden kann, da beide die gleiche Bemessungsgrundlage $(t-b) H$ haben.

Wenden wir uns nun einem Stpfl. zu, dessen Risikoaversion im Vergleich zu dem erwarteten Ertrag der Hinterziehung von Einkommensteuern so klein ist, daß er alle Zinsen hinterzieht. Dieser Stpfl. wird zwar auch mit einer marginal höheren Quellensteuer keine Zinsen deklarieren. Es kann sich aber die Ersparnis $l_{1}-C$ ändern, so daß das hinterzogene Einkommen $H=r\left(l_{1}-C\right)$ variiert. Im folgenden wird $\partial C / \partial b$ bestimmt. Die Reaktion des hinterzogenen Einkommens auf eine höhere Belastung an der Quelle folgt dann sofort als $\partial H / \partial b=-r \partial C / \partial b$.

Es wird statt $U\left(C_{1}, C_{2}\right)$ zunächst die additiv separable Nutzenfunktion $V\left(C_{1}\right)+$ $W\left(C_{2}\right)$ zugrundegelegt. Die Ecklösung ist damit durch

$$
\begin{aligned}
& V^{\prime}(C)-(1+r(1-t))\left(q W^{\prime}(G)+p W^{\prime}(P)\right)-\lambda r=0 \\
& q W^{\prime}(G)(t-b)-p W^{\prime}(P)(t-b) \alpha-\lambda=0 \\
& r\left(l_{1}-C\right)-H=0 \\
& \lambda>0
\end{aligned}
$$

${ }^{4}$ Der Fall $\lambda=0$ und $r\left(l_{1}-C\right)=H$ ist möglich, aber unwahrscheinlich und wird vernachlässigt. 
charakterisiert. Einsetzen von (4.6) und (4.7) in (4.5) liefert

$$
\begin{aligned}
& V^{\prime}(C)-(1+r(1-b)) q W^{\prime}\left((1+r(1-b))\left(l_{1}-C\right)+l_{2}\right) \\
& -(1+r(1-t-(t-b) \alpha)) \times \\
& p W^{\prime}\left((1+r(1-t-(t-b) \alpha))\left(l_{1}-C\right)+l_{2}\right)=0
\end{aligned}
$$

Diese Gleichung bestimmt das optimale $C=C(b)$ für den Fall eines Stpfl., der alle Zinsen hinterzieht.

Setzt man $C(b)$ in (4.9) ein, ergibt sich die Identität $I(b, C(b)) \equiv 0 . \quad$ Die gesuchte Ableitung ist

$$
\frac{\partial C}{\partial b}=-\left(\frac{\partial I}{\partial C}\right)^{-1} \frac{\partial I}{\partial b}
$$

Das Vorzeichen von $\partial I / \partial C$ ist negativ. Damit hängt die Richtung der Reaktion des Konsums der ersten Periode von $\partial I / \partial b$ ab. Es gilt

$$
\begin{aligned}
\frac{\partial I}{\partial b}= & r\left[q W^{\prime}(G)-p W^{\prime}(P) \alpha\right] \\
& +r\left(l_{1}-C\right)\left[(1+r(1-b)) q W^{\prime \prime}(G)\right. \\
& \left.-(1+r(1-t-(t-b) \alpha)) p W^{\prime \prime}(P) \alpha\right]
\end{aligned}
$$

Der Term in der ersten eckigen Klammer ist gleich $\lambda /(t-b)>0$, wie man (4.6) und (4.8) entnehmen kann. Das Vorzeichen des Ausdrucks in der zweiten eckigen Klammer kann nicht bestimmt werden. Selbst Annahmen über die absolute Risikoaversion $-W^{\prime \prime} / W^{\prime}$ helfen nicht weiter. Daher läßt sich nicht mehr als $\partial C / \partial b \gtreqless 0$ feststellen. Folglich kann die Erhöhung der Quellensteuer sowohl zu einem Sinken als auch zu einem Steigen der deklarierten Zinserträge führen. Dies gilt auch für die Einführung einer Quellensteuer, wie man sich durch einen Blick auf (4.10) leicht überzeugen kann.

Eine Nutzenfunktion, die nicht additiv separabel ist, führt nicht dazu, daß das Ergebnis an Bestimmtheit gewinnt, da schon ihre Kreuzableitungen im allgemeinen nicht bestimmt werden können.

Der Ersatz der Straffunktion $\Phi_{1}$ durch die Geldstrafe nach deutschem Recht kann nicht zu besseren Ergebnissen führen. Denn es tritt ein zusätzlicher Effekt auf, der per se dafür sorgt, daß das Verhalten des Stpfl. nicht prognostiziert werden kann: Spart er z.B. mehr, steigt mit dem höheren Einkommen der Strafsatz $\gamma\left((1-t) r\left(l_{1}-C\right)+l_{2}\right)$. Das macht es jedoch weniger attraktiv, zu sparen und die Zinsen zu hinterziehen.

Dieser Abschnitt hat gezeigt, daß man auch mit einem endogenen Einkommen für beide Formen der Geldstrafe dieselben Resultate gewinnt. Dies ist die Ausnahme in der Theorie der Steuerhinterziehung - im allgemeinen kommt man mit unterschiedlichen Straffunktionen zu verschiedenen Ergebnissen. Der Grund für 
die Übereinstimmung ist die gemeinsame Bemessungsgrundlage der beiden hier behandelten Strafen.

Es waren zwei Klassen von Stpfl. zu unterscheiden, je nachdem ob eine Ecklösung oder eine innere Lösung bezüglich der Variablen $H$ vorlag. Will man das Modell für die wirtschaftspolitische Diskussion auswerten, bleibt die Frage offen, welcher Fall denn der "relevantere" ist. Angesichts des begrenzten statistischen Materials zur Frage der steuerlichen Erfassung von Zinsen kann man nur die Antwort geben, daß keiner der beiden Fälle als irrelevant ausgeschieden werden kann ${ }^{5}$. Die Analyse der Aufkommenswirkung der Quellensteuer im nächsten Abschnitt knüpft daher an die getroffene Fallunterscheidung an.

\subsection{Die Aufkommenswirkung der Quellensteuer und ihr Beitrag zu einer gleichmäßigeren Besteuerung}

In der Bundesrepublik ist die Quellensteuer auf Zinserträge eingeführt worden, um die durch die Tarifreformen 1986 bis 1990 erwarteten Einnahmeausfälle zu einem Teil auszugleichen. Eine höhere Belastung der festverzinslichen Anlagen sollte gleichzeitig zu einer gleichmäßigeren Besteuerung von Lohn- und Kapitaleinkommen führen. Es ist fraglich, ob die Quellensteuer diesen beiden Aufgaben hätte gerecht werden können. Diese Beurteilung wird durch die Auswertung des Modells in bezug auf das erwartete Steueraufkommen begründet.

Zunächst ist zu präzisieren, was im folgenden unter einer gleichmäßigen Besteuerung verstanden werden soll. Wenn je zwei Stpfl. mit dem gleichen Einkommen vor Steuer eine gleich hohe erwartete Steuerlast tragen, werden sie gleichmäßig besteuert. Zu dieser Definition sind zwei Bemerkungen angebracht. Wenn die Steuerschuld zumindest eines der beiden Stpfl. eine Zufallsvariable ist, sind die Steuerbelastungen nicht ohne weiteres vergleichbar. Ich schlage daher vor, die erwartete Steuerlast als Vergleichsmaßstab zu wählen. Zweitens stellt die Definition auf die horizontale Gleichheit in bezug auf die Besteuerung des Periodeneinkommens ab, da die Quellensteuer mit dieser Vorstellung von Gerechtigkeit begründet wird und ich sie an ihrem eigenen Anspruch messen will. Die Frage, ob es nicht (horizontal) gerechter wäre, Kapitalerträge von Haushalten steuerlich zu befreien und das Lebenseinkommen zu besteuern, wird im nächsten Kapitel verfolgt.

Vergleichen wir Hauhalte mit identischem Einkommen vor Steuer, aber unter-

\footnotetext{
${ }^{5}$ Krischausky (1988), der die gründlichste Schätzung der insgesamt hinterzogenen Zinserträge vorgelegt hat, gibt keine Antwort darauf, wie die Haushalte auf diese zwei Klassen aufgeteilt sind.
} 
schiedlichen Anteilen von Zins- und Lohneinkommen, so bevorzugt die geltende Besteuerung die Haushalte mit den größeren Zinseinkommen. Sie tragen die geringere erwartete Steuerlast. Was ändert eine Quellensteuer auf alle Anlagen an dieser Situation? Sie berührt die Lohneinkommen nicht. Sie führt also zu einer gleichmäßigeren Besteuerung, wenn - bei gegebenem Einkommen - die erwartete Steuerlast auf Zinserträge steigt. Die Auswertung des Modells in bezug auf das erwartete Aufkommen pro Sparer aus der Besteuerung von Zinserträgen wird daher sowohl die Fragen nach der fiskalischen Ergiebigkeit der neuen Steuer als auch nach ihrem Beitrag zu einem Mehr an Steuergerechtigkeit beantworten.

$\mathrm{Da}$ das oben angegebene Kriterium einer gleichmäßigen Besteuerung ein gegebenes Einkommen voraussetzt, gehen wir von einer exogenen Ersparnis $s$ aus. Das erwartete Aufkommen $A$ aus der Quellen-, der Einkommensteuer und der Geldstrafe ist gleich

$$
\begin{aligned}
A & =b r s+q(t-b)(r s-H)+p(t-b)(r s+\alpha H) \\
& =t r s-(q-p \alpha)(t-b) H
\end{aligned}
$$

da die Bank den abzuziehenden Betrag brs in voller Höhe an den Fiskus weiterleitet 6. Es wäre ein Fehler, das Aufkommen der Quellensteuer brs isoliert zu betrachten. Denn sie beeinflußt die sich aus der Veranlagung zur Einkommensteuer ergebenden Zahlungen und die Geldstrafe. Es müssen vielmehr alle mit der Besteuerung der Zinserträge zusammenhängenden Zahlungen betrachtet werden.

Der Fall eines Stpfl. mit einer inneren Lösung bezüglich $H$ zeigt dies ganz deutlich. Er kompensiert die Quellensteuer durch eine geringere Deklaration von Zinseinkommen. Mit einer höheren Quellensteuer steigt zwar das Aufkommen dieser selbst, die abschließende Einkommensteuerzahlung aber verringert sich um den gleichen Betrag. Mit $\partial H / \partial b=H /(t-b)$ erhalten wir $\partial A / \partial b=0$. Die Aufkommenswirkung der Quellensteuer ist gleich null ${ }^{7}$. Sie führt nicht zu einer höheren Belastung der Zinseinkommen.

Für die Frage einer gleichmäßigeren Besteuerung von Kapital- und Lohneinkommen ergibt sich direkt eine Schlußfolgerung. Da die Quellensteuer die Zinseinkommen nach Steuer und Strafe nicht berührt, ändert sich die relative Belastung der beiden Einkommensquellen nicht. Die Einführung der Quellensteuer ändert nichts daran, daß gleiche - gemessen an dem Einkommen vor Steuer - ungleich behandelt werden. Ganz unabhängig davon jedoch, was man unter Steuergerechtigkeit verstehen will, gilt: wenn sich nichts ändert, kann es nicht zu einem Mehr

\footnotetext{
${ }^{6}$ Yaniv (1988) gibt für den Fall einer Lohnsteuer die Annahme auf, daB sie von dem zum Abzug verpflichteten Unternehmen nicht zum Teil hinterzogen wird (siehe dazu auch Hagedorn, 1989).

${ }^{7}$ Eine höhere Belastung der Reingewinne des Investors aus Abschnitt 4.4 ergibt dagegen ein höheres erwartetes Aufkommen $t(E(X)-r) a$, wobei $E(X)$ der Erwartungswert von $X$ ist.
} 
an Gerechtigkeit kommen.

Kommen wir zu der Ecklösung für den Fall einer gegebenen Ersparnis. Das Aufkommen kann nun als

$$
A=b r s+p(t-b)(1+\alpha) r s
$$

geschrieben werden. Es entwickelt sich bei einer Erhöhung von $b$ wie folgt:

$$
\frac{\partial S}{\partial b}=(1-p(1+\alpha)) r s=(q-p \alpha) r s>0
$$

Die Belastung der Zinsen steigt mit der Quellensteuer. Sie führt bezüglich der Stpfl., die alle Zinsen hinterziehen, zu einer gleichmäßigeren Besteuerung, wenn das Einkommen vor Steuer gegeben ist.

Diese Voraussetzung ist jedoch problematisch. Die Stpfl. können der Steuer ausweichen, indem sie weniger sparen. In dem Rest dieses Abschnitts wird daher die Aufkommenswirkung der Quellensteuer in dem Modell mit endogenen Zinseinkommen betrachtet. Für den Fall einer inneren Lösung ist das Ergebnis schnell gefunden: Das erwartete Aufkommen bleibt konstant, da sich die Ersparnis und die hinterzogene Steuer nicht ändern.

Betrachten wir jetzt die Ecklösung. Mit $\Phi_{1}$ ist das Aufkommen

$$
A=b r\left(l_{1}-C\right)+p(t-b)(1+\alpha) r\left(l_{1}-C\right)
$$

Es entwickelt sich bei einer Erhöhung der Steuer wie folgt:

$$
\begin{gathered}
\frac{\partial A}{\partial b}=r\left(l_{1}-C\right)-b r \frac{\partial C}{\partial b}-p(1+\alpha)\left[r\left(l_{1}-C\right)+(t-b) r \frac{\partial C}{\partial b}\right] \\
=r\left(l_{1}-C\right)(1-p(1+\alpha))-(b r+p(1+\alpha)(t-b) r) \frac{\partial C}{\partial b}
\end{gathered}
$$

Wegen der Voraussetzung $q-p \alpha=1-p(1+\alpha)>0$ ist der erste Summand positiv. Das Vorzeichen des zweiten Summanden hängt von der Reaktion der Ersparnis ab. Nur mit $\partial C / \partial b \leqq 0$ ließe sich die Entwicklung des Aufkommens bestimmen. Das zusätzliche Aufkommen der Quellensteuer isoliert betrachtet würde in diesem Fall die (nicht bestimmbare) Änderung der abschließenden Einkommensteuer- und Strafzahlung übertreffen. Die Quellensteuer würde über höhere Zinseinkommen zu insgesamt höheren Steuern aus Zinserträgen führen. Mit $\partial C / \partial b>0$ bleibt die Aufkommenswirkung ungewiß. Denkbar ist es jedenfalls, daß der öffentlichen Hand Einnahmeausfälle entstehen.

Die Quellensteuer kann bei endogenen Zinseinkommen dazu führen, daß die (erwartete) Steuerlast eines Stpfl., der alle Zinsen hinterzieht, sinkt. Ob sie damit zu einem Mehr an Steuergerechtigkeit führt, muß dahingestellt bleiben. Es ist fraglich, ob das oben angegebene Kriterium steuerlicher Gleichmäßigkeit anwendbar ist, wenn das Einkommen mit der Steuer variiert. 
Mit der Strafe nach deutschem Recht ergibt sich im Fall der Ecklösung aus der Besteuerung von Zinsen das Aufkommen

$$
A=b r\left(l_{1}-C\right)+p(t-b)\left[1+\gamma\left((1-t) r\left(l_{1}-C\right)+l_{2}\right)\right] r\left(l_{1}-C\right)
$$

Die gesuchte Ableitung ist

$$
\begin{aligned}
& \frac{\partial A}{\partial b}=r\left(l_{1}-C\right)\left[1-p\left(1+\gamma\left((1-t) r\left(l_{1}-C\right)+l_{2}\right)\right)\right] \\
& -\left[b r+p r(t-b)\left(1+\gamma\left(2(1-t) r\left(l_{1}-C\right)+l_{2}\right)\right)\right] \frac{\partial C}{\partial b}
\end{aligned}
$$

Für die Strafe nach deutschem Recht muß $q-p \gamma\left((1-t) r\left(l_{1}-C\right)+l_{2}\right)>0$ vorausgesetzt werden, damit das Einkommen nicht voll deklariert wird. Gilt $\partial C / \partial b \leqq 0$, ist der zweite Summand in (4.11) nichtnegativ. Da der erste Term positiv ist, ergibt sich - wie schon mit der Strafe $\Phi_{1}$ - eine positive Aufkommenswirkung der Quellensteuer. Gegenüber $\Phi_{1}$ erhalten wir hier nur wieder den zusätzlichen Effekt einer mit dem Einkommen steigenden Strafzahlung. Mit $\partial C / \partial b>0$ kann freilich wieder nichts ausgesagt werden. Da die Reaktion der Ersparnis in Abschnitt 4.5 für die Ecklösung nicht bestimmt werden konnte, muß die Aufkommenswirkung der Quellensteuer ungewiß bleiben.

Wir haben die Anleger unter den Haushalten einer Wirtschaft in zwei Gruppen geteilt und jeweils ein repräsentatives Mitglied analysiert. Man könnte nun versuchen, die relative Größe der Gruppen zu bestimmen. Eine kleine Wahrscheinlichkeit etwa, steuerlich geprüft zu werden, macht die Hinterziehung attraktiv und würde zu einer relativ großen Gruppe derjenigen führen, die die Ecklösung wählen. Wäre das Verhalten jeder Gruppe bekannt, könnte aufgrund der relativen Größe beider Gruppen die Entwicklung des Aufkommens insgesamt geschätzt werden. Da die Reaktion einer der beiden Gruppen auf die Quellensteuer jedoch nicht vorhersagbar ist, nützt dieses Verfahren nichts. Es kann hier nur noch einmal wiederholt werden, $\mathrm{da} B$ von der Quellensteuer nicht ohne weiteres erwartet werden kann, daß sie insgesamt höhere Steuereinnahmen bewirkt - auch wenn die neue Steuer isoliert betrachtet einen positiven Beitrag leistet.

\subsection{Progressiver Tarif}

Die direkt progressive Steuerbetragsfunktion T(B) der Bemessungsgrundlage $B>$ 0 sei definiert durch:

$$
\begin{aligned}
B>T(B) & >0 \\
1>T^{\prime}(B) & >b \\
T^{\prime \prime}(B) & >0
\end{aligned}
$$


Welche Auswirkung hat die Quellensteuer, wenn das Einkommen statt einem proportionalen einem direkt progressiven Tarif unterliegt? Um diese Frage zu beantworten, müssen die Budgetgleichungen für die zwei möglichen Zustände abgeleitet werden. Ich gehe wie in den Abschnitten 4.2 und 4.3 von einer gegebenen Ersparnis aus.

Unter der Bedingung, daß der Stpfl. einer steuerlichen Prüfung entgeht, ist sein Konsum gleich

$$
\begin{aligned}
G & =(1+r(1-b)) s+w-T(w+r s-H)+b(r s-H) \\
& =(1+r) s+w-T(w+r s-H)-b H
\end{aligned}
$$

Betrachten wir die erste Zeile. Die Zinszahlungen sind um die Quellensteuer gekürzt. Von der progressiven Steuer auf der Grundlage des Lohneinkommens und der deklarierten Zinserträge kann die vorweg durch die Bank bezahlte Quellensteuer abgezogen werden. Der Abzug ist nur entsprechend der Höhe der versteuerten Zinsen möglich. Das Lohneinkommen kann, so sei angenommen, nicht hinterzogen werden, da es der Lohnsteuer unterliegt.

In dem weniger glücklichen Zustand einer steuerlichen Prüfung ist die hinterzogene Steuer wie unter dem proportionalen Tarif die Ausgangsgröße der Strafe. Es wird ein konstanter Strafsatz vorausgesetzt. Wird der Stpfl. kontrolliert, gilt die Beschränkung

$$
P=(1+r) s+w-T(w+r s)-\alpha(T(w+r s)-T(w+r s-H)-b H)
$$

Die mit $\alpha$ multiplizierte Klammer enthält die hinterzogene Steuer. Es gibt ein einfach zu erzeugendes Indiz, das auf eine korrekte Formulierung der Beschränkungen $G$ und $P$ hinweist. Wäre der Stpfl. steuerehrlich, müßte sein Konsum in beiden Zuständen gleich sein. In der Tat ist mit $H=0$ auch $G=P$.

Das Optimierungsproblem wird für den progressiven Tarif nicht explizit formuliert. Wir fragen stattdessen, unter welcher Bedingung sich der Konsum trotz einer Erhöhung der Quellensteuer nicht ändert. Wann sind also

$$
\frac{\partial G}{\partial b}=T^{\prime}(w+r s-H) \frac{\partial H}{\partial b}-H-b \frac{\partial H}{\partial b}=0
$$

und

$$
\frac{\partial P}{\partial b}=-\alpha \frac{\partial G}{\partial b}=0
$$

erfüllt? Die Ableitung der Nachfragefunktion $H(b)$ ist unter der Voraussetzung einer inneren Lōsung

$$
\frac{\partial H}{\partial b}=\frac{H}{T^{\prime}(w+r s-H)-b}
$$

und stellt sicher, daß $G$ und $P$ sich nicht mit $b$ ändern. 
Das hinterzogene Einkommen steigt mit der Quellensteuer. Für eine quantitative Vorhersage muß man den marginalen Einkommensteuersatz in dem Zustand ohne eine Prüfung kennen und wissen, in welchem Umfang in der Ausgangssituation Zinsen nicht deklariert werden. Unter einem direkt progressiven Tarif ist die Reaktion des Stpfl. ähnlich einfach wie bei einer proportionalen Steuer. Die hinterzogenen Steuern bleiben jedesmal konstant, wenn der Haushalt den abgeleiteten Regeln folgt.

Das gleiche Ergebnis erhält man, wenn die Ersparnis endogen ist. Das entsprechende Argument enthält bereits Abschnitt 4.5. Die deutsche Straffunktion ändert ebenfalls nichts.

Die progressive Besteuerung führt im Fall endogener Zinsen und einer Ecklösung wieder zu unbestimmten Ergebnissen. Der Effekt der Quellensteuer auf die Ersparnis ist nicht eindeutig. Die Entwicklung des Aufkommens von den Anlegern, die keine Zinsen deklarieren, läßt sich folglich nicht vorhersagen.

\subsection{Abschließende Bemerkungen}

Dieses Kapitel hat die Wirkungen einer Quellensteuer auf Zinserträge unter der Annahme untersucht, daß die Sparer ihr Geld nur in eine einzige quellenbesteuerte Anlage investieren können. Von quellensteuerfreien In- und Auslandsanlagen wurde abgesehen. Trotz dieser günstigen Voraussetzungen erfüllt die Quellensteuer die Erwartungen nicht, die unter ungünstigeren Bedingungen in sie gesetzt worden sind. Ein Steuerpflichtiger kann der Steuer voll ausweichen, wenn er bislang nicht seine gesamten Zinserträge hinterzogen hat. Er leistet keinen Beitrag zu einem höheren Aufkommen aus der Besteuerung der Kapitalerträge. Die Quellensteuer führt folglich nicht dazu, daß alle Bezieher von Zinseinkommen den Beziehern von Lohneinkommen (zumindest der Tendenz nach) gleichgestellt werden. Die Steuer trifft nur die Anleger, die ihre gesamten Zinserträge hinterziehen ${ }^{8}$. Diese Gruppe reagiert möglicherweise durch eine geringere Sparbereitschaft auf die Belastung der Erträge an der Quelle. In diesem Fall ist es sogar denkbar, daß die Steuer einen Rückgang des Aufkommens aus der Besteuerung von Kapitaleinkommen verursacht. Die Wirkung der Quellensteuer auf die Sparbereitschaft der Anleger, die die Ecklösung wählen, ist letztlich jedoch eine empirische Frage ${ }^{9}$.

War es also richtig, die Quellensteuer nach einer Laufzeit von einem halben Jahr abzuschaffen, wenn sie selbst ohne Ausnahmeregelungen und ohne die Möglichkeit des Kapitalexports in ein quellensteuer- und kontrollmitteilungenfreies Aus-

\footnotetext{
${ }^{8}$ Die These von Schneider (1987, S. 2533), "Die Quellensteuer trifft steuerunehrliche Inhaber zinstragender Forderungstitel", ist daher zu einfach.

${ }^{9}$ Geiger (1989, S. 332) z.B attestiert den Bundesbürgern eine ungebrochene Sparbereitschaft.
} 
land die in sie gesetzten Erwartungen nicht (theoretisch abgesichert) hätte erfüllen können? Dazu ist trotz allem Respekt vor der bewährten Methode der komparativen Statik festzustellen: Man kann nicht alle Änderungen mit " -1 " multiplizieren und erwarten, wieder in den Zustand vor der Reform zurückzukehren. Im Fall der Quellensteuer bleibt ein Verlust an Glaubwürdigkeit der Steuerpolitik übrig mit der Folge, daß es in Zukunft (noch) weniger ehrliche Steuerzahler geben wird (Tipke, 1989, S. 299, Rürup, 1989, S. 336). Wenn der Staat nicht einmal eine bescheidene Quellensteuer von $10 \%$ auf Zinsen durchsetzen kann, werden sich viele Steuerpflichtige fragen, warum sie ihre Einkünfte ordnungsgemäß angeben sollten. Daß der Bundesgerichtshof im Juni 1989 die im Vergleich zu betimmten anderen Einkünften unterschiedliche Erhebung der Steuern auf Zinsen als verfassungskonform eingestuft hat, läßt nicht darüber hinwegsehen, daß die Finanzpolitik von ihr selbst formulierte Probleme nicht löst, sondern vor ihnen in der Erwartung kapituliert, daß sich die Steuerreform von selbst finanziert. "Der Gesetzgeber hat entgegen allen Bekundungen das Interesse an Steuergerechtigkeit bei der Behandlung von Zinseinkünften verloren" (Rürup, 1989, S. 337).

Die Einführung der Quellensteuer war vermutlich eine wenig geeignete Maßnahme, um ein höheres Aufkommen und eine gleichmäßigere Besteuerung aller Einkommen zu erreichen. Ihre Abschaffung nach einem halben Jahr war ebenfalls keine zukunftsweisende Entscheidung. Erstens hätte man eine längere Zeit gebraucht, um die Wirkungen dieser Steuer auch empirisch beurteilen zu können. Zweitens ist es immer besser, Verbesserungen oder Alternativen anzubieten, statt einen ungeordneten Rückzug anzutreten. Eine Alternative gab es, die brauchbarer gewesen wäre: Stichproben von Sparkonten. Diese Maßnahme ist freilich wie die Quellensteuer dem Einwand ausgesetzt, sie würde die Kapitalströme in das Ausland lenken (Geiger, 1989, S. 334). Daher wird in dem nächsten Kapitel dieser Arbeit der Versuch unternommen, eine Alternative zu der gegenwärtigen Form der Haushaltsbesteuerung zu skizzieren, die weniger Probleme schaffen würde. 


\section{Kapitel 5}

\section{Steuerhinterziehung und Bankgeheimnis unter einer direkten Konsumsteuer}

\subsection{Einleitung}

Die Zinserträge der privaten Haushalte werden steuerlich zu einem großen Teil nicht erfaßt. Der Versuch, an dieser Situation mit Hilfe einer Quellensteuer auf Zinserträge etwas zu ändern, ist gescheitert. $\mathrm{Ob}$ andere Maßnahmen in dieser Richtung zu erwarten sind, ist fraglich. Da Zinsen Teil des Einkommens sind, kann die Finanzwissenschaft nur noch schlechten Gewissens lehren, das Einkommen sei in der Bundesrepublik Bemessungsgrundlage der direkten Besteuerung. Der Vorschlag, die Zinserträge steuerlich zu befreien und die Einkommen durch eine direkte Konsumsteuer zu ersetzen, liegt daher nahe ${ }^{1}$. Gegen diese Substitution spricht aber die von vielen Autoren vertretene Ansicht, eine solche persönliche Ausgabensteuer sei nicht praktikabel.

In diesem Kapitel werden zwei Thesen vertreten: Unter den heute gegebenen institutionellen Verhältnissen auf dem Gebiet der Steuererhebung - unter anderem $\S 30$ a der Abgabenordnung, der die Ausfertigung von Kontrollmitteilungen über die Ersparnisse und Zinserträge der Haushalte untersagt - ist eine Konsumsteuer einfacher zu administrieren als die Einkommensteuer. Insbesondere stellt die durch das Bankgeheimnis ermöglichte Steuerhinterziehung unter einer Konsumsteuer kein Problem dar. Zweitens führt die Konsumsteuer - wieder gegeben

\footnotetext{
${ }^{1}$ Eine indirekte Konsumsteuer gibt es schon in Form der Umsatzsteuer, die ein anerkannter Bestandteil unseres Steuersystems ist. Da diese nur mit einem proportionalen Tarif praktikabel ist, ist die direkte Ausgabensteuer dann sinnvoll, wenn der Konsum einem progressiven Tarif unterliegen soll.
} 
das Bankgeheimnis bzw. der Schutz der Bankkunden vor dem Fiskus - eher als die existierende Einkommensteuer zu einer gleichmäßigen Besteuerung. Insbesondere tragen Steuerpflichtige mit zeitlich schwankendem Konsum auch bei progressivem Tarif keine höhere Steuerlast als solche mit gleichmäßigen Ausgaben.

$\mathrm{Da}$ in der Literatur unumstritten ist, daß für Zwecke der direkten Besteuerung der Konsum nicht direkt, sondern nur indirekt als Einkommen minus (Netto-) Ersparnis ermittelt werden kann, klingen die zwei Thesen nicht sehr einleuchtend, da der Fiskus die Höhe der Ersparnisse eines konsumsteuerpflichtigen Haushalts in vielen Fällen nicht festzustellen vermag und die Ausgabensteuern durch Übertreibung der Ersparnisse hinterzogen werden könnten. Da aber neben Kontrollen und Strafen und dem Abzug an der Quelle als drittes denkbares Instrument zur Durchsetzung eines Steueranspruchs das logische Schließen der Finanzverwaltung berücksichtigt werden muß, kann diese Behörde unter bestimmten Bedingungen trotz beschränkter Informationen über die Sparkonten der Steuerzahler ihrem Auftrag gerecht werden. Diese Möglichkeit wird in Abschnitt 5.3 mit Hilfe eines einfachen Modells eines repräsentativen Sparers illustriert. Fragen der horizontalen und vertikalen Steuergerechtigkeit werden ebenfalls in Abschnitt 5.3 aufgegriffen. Abschnitt 5.4 diskutiert, ob die zwei oben angegebenen Thesen unter allgemeineren Voraussetzungen zutreffend sein können. Bevor im Detail auf die Konsumsteuer eingegangen wird, zeigt in Abschnitt 5.2 eine Analyse der jüngsten steuerpolitischen Ereignisse, daß der Übergang zu einer direkten Konsumsteuer vielleicht der einzige Weg aus der Sackgasse ist, in die die "Erfassung" der Zinserträge geraten ist. Insgesamt soll das Kapitel eine Ergänzung zu den administrativen Aspekten der Vorschläge zur Einführung einer Konsumsteuer ${ }^{2}$ sein und die Frage der steuerlichen Gleichmäßigkeit unter einer Konsumsteuer beleuchten.

\subsection{Erfassung der Zinserträge oder direkte Besteuerung des Konsums?}

Um die steuerliche Erfassung der Zinserträge zu verbessern, sind 1989 drei Maßnahmen eingeführt worden. Es wurde eine Quellensteuer von $10 \%$ auf inländische Zinserträge eingeführt; der sogenannte Bankenerlaß - ursprünglich eine Verwaltungsanweisung, die Stichproben von Konten ohne konkreten Verdacht untersagte

\footnotetext{
${ }^{2}$ Neben Kaldors Klassiker von 1954 sind drei Gutachten jüngeren Datums zu nennen, die im Regierungsauftrag in Schweden, England und den Vereinigten Staaten erstellt wurden und zu einem positiven Votum für eine persönliche Ausgabensteuer kamen (Lodin, 1982, MeadeKommission, 1978, Bradford, 1984). Zu den administrativen Problemen einer Konsumsteuer bieten neben diesen Arbeiten Graetz (1980), Mieszkowski (1980) und Peffekoven (1980, S. 420 427) einen guten Überblick.
} 
und die Betriebsprüfer der Banken vergessen lassen sollte, was sie bei ihrer Arbeit über die Konten und Depots privater Haushalte erfuhren - wurde unter dem Titel "Schutz von Bankkunden" als $\S 30$ a in die Abgabenordnung übernommen; reuigen Stpfl., die Zinserträge vergangener Jahre nachträglich deklarierten, wurde die Nachzahlung der hinterzogenen Steuer erlassen ${ }^{3}$. Zu diesem Maßnahmenbündel, das ein Teil der "Steuerreform 1990" war, kann man einiges sagen. Fraglich ist insbesondere, ob die Quellensteuer geeignet zum Erreichen der angestrebten Ziele war (siehe Kapitel 4) und ob Detailprobleme gut gelöst waren. Nicht fraglich ist, daß das Bündel ein geradezu höflicher Versuch des Staates war, sich an den kontinuierlich steigenden Zinserträgen der privaten Haushalte zu beteiligen. Man hätte ja auch mit höheren Strafen und Stichproben oder gar flächendeckenden Kontrollmitteilungen arbeiten können.

Die Reaktionen der Stpfl. waren weniger höflich. Die "Steueramnestie" und die Festschreibung einer geringen Entdeckungswahrscheinlichkeit bei der Hinterziehung von Zinsen in $\S 30$ a AO wurden wie selbstverständlich hingenommen 4. Die Quellensteuer aber führte zu "Anpassungs- und Ausweichreaktionen der inländischen Anleger" (Deutsche Bundesbank, 1989, S. 49) und verbreitete eine Empörung, die eineinhalb Jahre lang den Wirtschaftsteil der Zeitungen füllen konnte. Die Ausweichreaktionen kann man leicht verstehen, wenn man davon ausgeht, daß Stpfl. den Erwartungsnutzen ihres Nettoeinkommens maximieren. Im übrigen ist der Widerstand der Zensiten gegen die Quellensteuer nicht so einfach zu verstehen. Drei mögliche Erklärungen bieten sich an:

- Die Sparer wollten bestehende steuerliche Privilegien verteidigen.

- Die Einführung der Quellensteuer wurde als Verletzung der Privatsphäre empfunden.

- Die Besteuerung von Zinsen aus der Anlage versteuerten (Lohn-) Einkommens wird als ungerecht angesehen.

Die erste Erklärung ist naheliegend und sicher ein Teil der Wahrheit. Ihr folgt die - für Fragen der Steuererhebung und -gerechtigkeit nicht verantwortliche Bundesbank, wenn sie feststellt, die "Quellensteuer auf inländische Zinseinkünfte

\footnotetext{
${ }^{3}$ Auch vorher schon zog eine Nachdeklaration keine Strafe nach sich. Die Selbstanzeige nach $\$ 371 \mathrm{AO}$ hat nur die Nachzahlung der hinterzogenen Steuer zur Konsequenz.

${ }^{4}$ Ein gewisser Spielraum bezüglich der Kontrolle von Konten bleibt der Steuerfahndung. Einzelauskunftsersuchen sind in $\S 30$ a Abs. 5 AO ausdrücklich zugelassen. Die Ausschreibung von Kontrollmitteilungen soll nach $\S 30$ a Abs. 3 Satz 2 zwar unterbleiben. "Eine derartige Untersagung ist jedoch gesetzeswidrig und unwirksam, wenn sie der finanzbehördlichen Verpflichtung zur Sachverhaltsaufklärung zuwiderläuft" (Bilsdorfer, 1989, S. 85). Zu dem Spielraum, der für die Prüfung von Konten bleibt, siehe auch Kottke (1989). Inwieweit der gesetzlich zulässige Spielraum genutzt oder nicht genutzt wird, ist natürlich eine zweite Frage.
} 
wurde von Anlegern im In- und Ausland als einschneidende Verschlechterung der steuerlichen Rahmenbedingungen für Kapitalanlagen in der Bundesrepublik empfunden" (Deutsche Bundesbank, 1989, S. 48 f.). Die Erklärung übersieht jedoch, daß die Quellensteuer Teil eines Reformpakets war, zu dem auch die Entlastung durch einen neuen Einkommensteuertarif gehörte. Sie müßte daher eine Begründung dafür mitliefern, warum die Bürger sich auf einzelne Bestandteile eines Pakets fixieren, das der Staat nur als Ganzes anbieten wollte.

Die zweite Erklärung für den Widerstand gegen die Quellensteuer ist nicht von der Hand zu weisen. Das Bankgeheimnis, der Schutz der Geschäftsbeziehungen der Geldinstitute mit ihren Kunden vor Einblicken Dritter, wird in der Bundesrepublik (und anderswo) fast als Grundrecht angesehen (vgl. Walter, 1985, S. 2 und 23). Die verbreitete Empfindung der Bürger, durch die neue Steuer werde ihre Privatsphäre verletzt, ist von der Sache her jedoch nicht gerechtfertigt. Dazu nur zwei Anmerkungen: Was unter personenbezogene Daten fällt, die vor dem Staat und anderen Dritten zu schützen sind, ist von Land zu Land verschieden und daher diskussionswürdig. In den Vereinigten Staaten z.B. gibt es kein Bankgeheimnis - aber auch keine Personalausweise und keine polizeiliche Meldepflicht. Zweitens erfährt der Fiskus im Zuge der Anrechnung der Quellensteuer bei der Einkommensteuerveranlagung nur das Geldvermögen und die Zinsen derjenigen Haushalte, die steuerehrlich sind - die übrigen werden sich ja die Quellensteuer nur zum Teil bzw. gar nicht anrechnen lassen, wie in Kapitel 4 gezeigt worden ist. Der tiefere Grund für die Empörung gegen die Quellensteuer liegt eher darin, daß die diffuse Wahrnehmung vieler Menschen, ihre Persönlichkeitsrechte seien von vielen Seiten her Gefahren ausgesetzt, sich plötzlich gegen eine vermeintliche Quelle der Bedrohung richtet, auf die man sich leicht einigen kann.

Ein in der Bevölkerung oft gehörtes Argument gegen die Quellensteuer ist die sogenannte Doppelbesteuerung der Ersparnis durch die Einkommensteuer. Zuweilen konnte man glauben, John Stuart Mill sei (wieder) zum Bestseller geworden. Er schrieb: “... when (income is (R.H.)) saved and invested ... it thenceforth pays income tax on the interest or profit which it brings, notwithstanding that it has already been taxed on the principal. Unless, therefore, savings are exempted from income tax, the contributors are twice taxed on what they save, and only once on what they spend. ... The difference thus created to the disadvantage of prudence and economy is not only impolitic but injust." (Mill, 1848, S. 813-814). Wenn man die "Erfassung" der Zinserträge mit Hilfe der Quellensteuer als ungerecht und politisch unklug bezeichnen will, muß man den Bürgern aber auch vorwerfen, zu kurz gedacht zu haben: Über den Widerstand gegen die Quellensteuer hinaus ist es zu einer konstruktiven öffentlichen Diskussion über die geeignete Bemessungsgrundlage der direkten Besteuerung nicht gekommen.

Die Koalition von Bürgern und Banken hat durch den Export von Kapital in 
das für den Fiskus schwer zugängliche Ausland die Rücknahme der Quellensteuer erreicht. Die Motive für den Widerstand gegen die neue Steuer wird die zukünftige Steuerpolitik ernst nehmen müssen. Das Bankgeheimnis - hier der Schutz der Konten vor dem Einblick des Fiskus - wird einen gewissen Stellenwert einnehmen. Die systematische Erhebung von Stichproben der Konten - eine Erhöhung der Entdeckungswahrscheinlichkeit - oder erhöhte Strafen werden nur schwer durchzusetzen sein. Das Argument der Doppelbesteuerung der Ersparnis und die mit ihm verbundene Forderung nach einer gleichmäßigen Besteuerung wird Beachtung finden müssen.

Der Spielraum für die Steuerpolitik ist eng geworden. Nur zwei Wege bleiben offen, wenn die Finanzpolitik nicht erneut den Unmut der Steuerzahler auf sich ziehen will: Man kann einen status quo beibehalten, der den Namen Einkommensteuer nicht verdient - wie eng man den Einkommensbegriff auch immer fassen will: Zinsen sind Einkommen - und der in bezug auf die steuerliche Gleichmäßigkeit nicht gut abschneidet, wie unten gezeigt werden wird. Oder man geht zu einer Konsumsteuer über, die nicht schlechter als das bestehende System der direkten Besteuerung ist, wahrscheinlich aber besser abschneiden würde.

\subsection{Konsumsteuer und Bankgeheimnis}

Allingham/Sandmo (1972) gehen davon aus, daß die Finanzbehörde ausschließlich von dem Stpfl. selbst Angaben über die Höhe seiner Steuerbemessungsgrundlage erhält, wenn sie keine Prüfung vornimmt. Im Fall einer Prüfung, die mit einer positiven Wahrscheinlichkeit eintritt, erfährt sie das gesamte Einkommen des Haushalts. Demgegenüber geht die folgende Analyse davon aus, daß der Fiskus durch Dritte über einen Teil der Einkünfte des Stpfl. (korrekt) in Kenntnis gesetzt wird. In bezug auf die Zinserträge und die Ersparnisse soll die Finanzbehörde jedoch allein auf die Angaben des Haushalts angewiesen sein: Es wird angenommen, daß der "Schutz von Bankkunden" soweit geht, daß auch bei Verdacht auf Steuerhinterziehung keine steuerliche Prüfung der Konten erfolgt. Bevor jedoch in Abschnitt 5.3.2 die Konsequenzen dieser Annahme untersucht werden, wird zum Vergleich die Situation dargestellt, in der der Fiskus über die steuerlich relevanten Verhältnisse der Sparer vollständig informiert ist.

\subsubsection{Die Konsumsteuer bei vollständiger Information des Fiskus}

Der Haushalt lebt zwei Perioden und hat die Nutzenfunktion $U\left(C_{1}, C_{2}\right)$, wobei $C_{i}$, $i=1,2$, der Konsum in den zwei Zeitabschnitten ist. $U$ habe eine abnehmende 
Grenzrate der Substitution zwischen $C_{1}$ und $C_{2}$ und zusätzlich die Eigenschaften

$$
U_{1}\left(0, C_{2}\right)_{\mid C_{2}>0}=U_{2}\left(C_{1}, 0\right)_{\mid C_{1}>0}=\infty
$$

wobei $U_{i}$ die erste Ableitung nach dem $i$-ten Argument ist. Der Konsum ist also in jeder Periode "wesentlich": Ist der Konsum in einer Periode positiv, so hätte der Haushalt auch in der anderen Periode gerne einen positiven Konsum. Dies ist eine für ein intertemporales Modell vernünftige Annahme.

In jeder Periode erhält der Haushalt ein exogenes Einkommen $w_{i}, i=1,2$. In Periode 1 legt er seine Ersparnis $S$ zu einem sicheren Zins $r$ über eine Periode an. Besteuert wird der Konsum jedes Jahres. Die Steuerbetragsfunktion im Jahr $i$ wird mit $T\left(B_{i}\right)$ bezeichnet. Die Bemessungsgrundlagen $B_{i}, i=1,2$, sind: $B_{1}=w_{1}-S$ und $B_{2}=w_{2}+(1+r) S$. Die Budgetbeschränkungen des Haushalts lauten damit:

$$
\begin{aligned}
& C_{1}=w_{1}-S-T\left(w_{1}-S\right) \\
& C_{2}=w_{2}+(1+r) S-T\left(w_{2}+(1+r) S\right)
\end{aligned}
$$

In den Gleichungen (5.1) und (5.2) wird genau genommen der Konsum inklusive der Steuer selbst besteuert: In Periode 1 etwa muß der Stpfl. von dem Betrag $w_{1}-S$ seinen Konsum und die Steuer auf der Basis $w_{1}-S$ bestreiten. Die direkte Konsumsteuer wird dagegen in der Literatur zumeist mit einer Steuerbetragsfunktion $K\left(C_{i}\right)$ auf der Basis des Konsums exklusive der Steuer selbst versehen. In diesem Fall hätte zum Beispiel die Budgetbeschränkung von Periode 1 die Form

$$
C_{1}+K\left(C_{1}\right)=w_{1}-S
$$

Es ist vom Ergebnis her jedoch gleichgültig, ob in einer Steuerbemessungsgrundlage die Steuer selbst enthalten ist oder nicht. Denn man kann zu einem gegebenen Tarif auf der Basis exklusive der Steuer genau einen Tarif auf der steuerinklusiven Basis finden, der stets zu einer gleich hohen Steuerbelastung führt. Das ist für eine proportionale Steuer bekannt (siehe z.B. Meade, 1978, S. 28 f.): Betrachten wir (5.1) und (5.3), so kann man $w_{1}-S$ mit einem Steuersatz von $50 \%$ oder $C_{1}$ mit einem Satz von $100 \%$ belasten. Es gilt aber auch für indirekt und direkt progressive Steuerbetragsfunktionen. Zusätzlich entsprechen sich die Eigenschaften der zwei belastungsgleichen Tarife. Es gilt $T^{\prime}, T^{\prime \prime}>0$, genau wenn $K^{\prime}, K^{\prime \prime}>0$ sind. Ist der eine Tarif direkt progressiv, hat auch der andere Tarif diese Eigenschaft. Anhang 5.3.1 geht darauf näher ein. Da die Konsumsteuer unten mit der Einkommensteuer verglichen wird, und diese auf einer Basis inklusive der Steuer berechnet wird, soll im folgenden der Tarif $\mathrm{T}$ verwendet werden.

Das Maximierungsproblem des Haushalts ist

$$
\max _{S} U\left(C_{1}, C_{2}\right)
$$


Abbildung 5.1: Lineare und progressive Konsumsteuer bei vollständiger Information des Fiskus

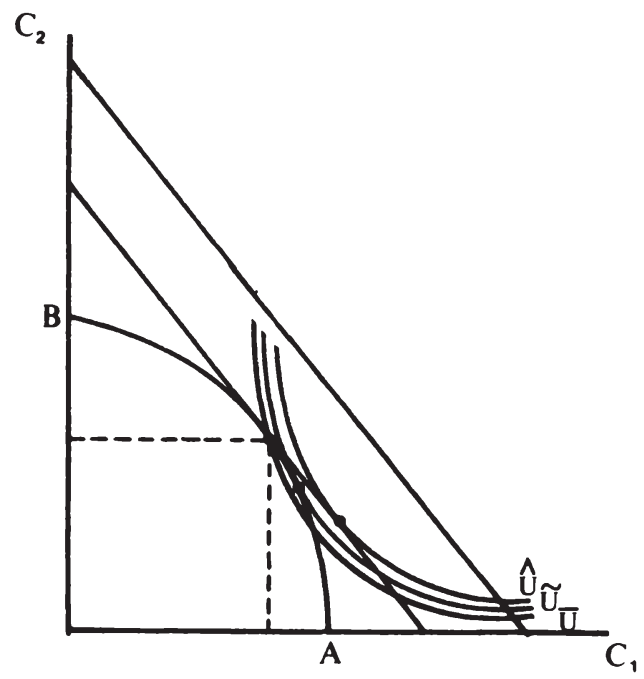

wobei $C_{1}$ und $C_{2}$ aus (5.1) und (5.2) eingesetzt werden. Die Bedingung erster Ordnung ist

$$
\begin{aligned}
& -U_{1}\left(C_{1}, C_{2}\right)\left(1-T^{\prime}\left(B_{1}\right)\right)+U_{2}\left(C_{1}, C_{2}\right)(1+r)\left(1-T^{\prime}\left(B_{2}\right)\right)=0 \\
& \Leftrightarrow \quad \frac{U_{1}}{U_{2}}=(1+r) \frac{1-T^{\prime}\left(B_{2}\right)}{1-T^{\prime}\left(B_{1}\right)}
\end{aligned}
$$

Abbildung 5.1 zeigt die Position des Haushalts für die zwei Fälle eines linearen Tarifs $T_{l}$ mit dem konstanten Grenzsteuersatz $T_{l}^{\prime}=t$ und eines direkt progressiven Tarifs $T_{d}$ mit $T_{d}^{\prime}, T_{d}^{\prime \prime}>0$. Die Gerade, die sich rechts in der Abbildung befindet, ist die Budgetbeschränkung $\left(C_{2}\right.$ als Funktion von $\left.C_{1}\right)$ vor Steuer. Die Gerade links ist die Beschränkung bei proportionalem Tarif der Konsumsteuer, die man aus (5.1) und (5.2) explizit berechnen kann. Beide Geraden haben die Steigung $-(1+r)$. Die strikt konkave Kurve $A B$ ist die Beschränkung bei direkt progressivem Tarif 5. Wählt der Stpfl. in jeder Periode einen gleich hohen Konsum, erreicht er das Nutzenniveau $\bar{U}$. Unter dem progressiven Tarif kann er in der Abbildung den höheren Nutzen $\tilde{U}$ erreichen, wenn er weniger spart oder sich höher verschuldet. Mit sinkender Ersparnis jedoch "kommt er in die Progression": Steigt $C_{1}$ um eine

\footnotetext{
${ }^{5}$ In der Abbildung ist für $B_{1}=B_{2}$ noch $T_{d}^{\prime}\left(B_{1}\right)=T_{d}^{\prime}\left(B_{2}\right)=t$ angenommen.
} 
kleine Einheit $d C_{1}$, muß die Ersparnis um

$$
d S=-\frac{1}{1-T^{\prime}\left(B_{1}\right)} d C_{1}
$$

sinken, wie man (5.1) entnehmen kann. Zusammen mit (5.2) folgt dann, daß $C_{2}$ um

$$
d C_{2}=-(1+r) \frac{1-T^{\prime}\left(B_{2}\right)}{1-T^{\prime}\left(B_{1}\right)} d C_{1}
$$

sinken muß. Diese Substitution von $C_{2}$ durch $C_{1}$ wird mit größerem Abstand zwischen $C_{2}$ und $C_{1}$ immer ungünstiger, da $T^{\prime}\left(B_{1}\right)$ mit sinkender Ersparnis steigt und $T^{\prime}\left(B_{2}\right)$ sinkt ${ }^{6}$. Eine entsprechende Argumentation gilt für die Haushalte, die lieber morgen als heute konsumieren. Eine allzu ungleichmäßige Verteilung des Konsums über die Zeit wird durch die direkt progressive Belastung des Periodenkonsums verhindert. Bei linearem Tarif ist das Austauschverhältnis zwischen $C_{2}$ und $C_{1}$ konstant gleich $1+r$. In Abbildung 5.1 erreicht der Stpfl. in diesem Fall das Nutzenniveau $\hat{U}>\tilde{U}$.

Bevor die Annahme aufgegeben wird, daß der Fiskus Einsicht in die Sparkonten hat, soll noch die Frage gestellt werden, ob die progressive Besteuerung des Periodenkonsums (Jahreskonsums) als horizontal gerecht angesehen werden kann. Die Antwort auf diese Frage dient zum Vergleich mit dem Fall, daß die Sparkonten durch das Bankgeheimnis geschützt sind. (i) Zwar finden sich Haushalte mit einem vor Steuer gleich hohen Lebenseinkommen (bezogen auf Periode 1)

$$
L_{1}=w_{1}+\frac{w_{2}}{1+r}
$$

auf der gleichen Budgetbeschränkung nach Steuer wieder ${ }^{7}$. Gleichen Wahlmöglichkeiten vor Steuer über Konsum in verschiedenen Jahren (d.h. gleichen intertemporalen Budgetbeschränkungen vor Steuer) entsprechen auch nach Steuer gleiche Konsummöglichkeiten. (ii) Haushalte aber, die einen ungleichmäßigen Ausgabenstrom planen, werden gegenüber Stpfl. mit einem gleichmäßigen Konsumstrom benachteiligt, da sie, wie oben gezeigt wurde, mit einem schlechteren Preisverhältnis $-d C_{2} / d C_{1}$ kalkulieren müssen.

Die erste dieser zwei Aussagen ist nicht offensichtlich und soll daher belegt werden. Hängt die Höhe des Konsums $C_{2}$ bei gegebenem $C_{1}$ von der Zusammensetzung des Lebenseinkommens ab? Führt also eine Änderung von $w_{1}$ und $w_{2}$, die

$$
0=d w_{1}+(1+r)^{-1} d w_{2}
$$

${ }^{6}$ Das heißt, die Kurve $A B$ ist wie oben behauptet strikt konkav.

${ }^{7}$ Eine Definition des Lebenseinkommens wird weiter unten gegeben. 
genügt, zu einem höheren Konsum in mindestens einer Periode? Für konstantes $C_{1}$ folgt aus (5.1)

$$
\begin{aligned}
0 & =\left(d w_{1}-d S\right)\left(1-T^{\prime}\left(B_{1}\right)\right) \\
\Leftrightarrow d S & =d w_{1}
\end{aligned}
$$

Aus (5.2) ergibt sich

$$
d C_{2}=\left(d w_{2}+(1+r) d S\right)\left(1-T^{\prime}\left(B_{2}\right)\right)
$$

Einsetzen von (5.7) und (5.6) führt zu $d C_{2}=0$. Hält man umgekehrt $C_{2}$ fest, folgt $d C_{1}=0$, wenn sich nur die Zusammensetzung, nicht aber die Höhe des Lebenseinkommens ändert.

\subsubsection{Hinterziehung von Konsumsteuern und Gleichmäßigkeit der Besteuerung}

Es soll jetzt der "Schutz von Bankkunden" durch $\S 30$ a AO berücksichtigt werden. Es wird angenommen, daß unrichtige Angaben der Haushalte über ihre Ersparnisse und Zinsen nicht aufgedeckt werden. Die Entdeckungswahrscheinlichkeit sei null. In dieser Form ist $\S 30$ a $\mathrm{AO}$ natürlich nicht formuliert. Die Abgabenordnung läßt einen gewissen Spielraum bezüglich der zulässigen Kontrollen von Konten (siehe oben Fußnote 4). Ich unterstelle im folgenden, daß die Finanzverwaltung an die untere Grenze dieses Spielraums geht.

Die Ersparnisse kennt der Fiskus nicht. Die exogenen Einkünfte $w_{i}$ sollten ihm jedoch in der Periode, in der sie ausgezahlt werden, zur Kenntnis kommen. Handelt es sich bei ihnen um Lohneinkünfte, läßt sich die Annahme gut begründen: Bei dem Wechsel von einer Einkommen- zu einer Konsumsteuer könnte die Lohnsteuer entsprechend dem Vorschlag von Mitschke (1985, S. 175) beibehalten werden. Im Rahmen der Konsumsteuerveranlagung am Ende jedes Jahres müßten Arbeitnehmer die Lohnsteuerkarte, auf der der Lohn und die Abzüge eingetragen sind, vorlegen; die Lohnsteuer würde dann auf ihre Konsumsteuerschuld angerechnet. Stellen die $w_{i}$ keine Lohneinkünfte, sondern andere Nicht-Zinseinkünfte dar, läßt sich ebenfalls begründen, warum sie dem Fiskus bekannt sein können, wenn man davon ausgeht, daß die heute bestehenden Kontrollen bei einem Übergang zu einer Konsumsteuer beibehalten oder intensiviert werden. Einkünfte aus Gewerbebetrieb, selbständiger Arbeit und Land- und Forstwirtschaft werden durch Betriebsprüfungen festgestellt. Auf Einkünfte aus Vermietung und Verpachtung könnte aus Grundbucheintragungen geschlossen werden. Die Erfassung dieser Einkünfte ist sicher unvollkommen. Immerhin existieren aber - verglichen mit der Erfassung der Zinsen - systematische Kontrollen (siehe allein die gesetzlichen 
Grundlagen über die Betriebs- oder Außenprüfung in den $\S \S 193-203$ AO), die leicht ausgebaut werden könnten.

Ist der Fiskus in bezug auf die Ersparnisse auf freiwillige Angaben angewiesen, ist der Konsum des Stpfl. in Periode 1 gleich

$$
C_{1}=w_{1}-S-T\left(w_{1}-S-F\right)
$$

$S+F$ ist die deklarierte Ersparnis. $F$ ist der Teil der deklarierten Ersparnis, der nur auf dem Formular für das Finanzamt existiert. Eine Erhöhung dieser fiktiven Ersparnis hat jedoch nach der Definition von $C_{1}$ die reale Konsequenz

$$
\frac{\partial C_{1}}{\partial F}=T^{\prime}\left(B_{1}\right)
$$

Der Konsum erhöht sich um die hinterzogene Steuer.

$\mathrm{DaB}$ die Konsumsteuer durch Übertreibung der Ersparnisse hinterzogen werden kann, ist von vielen Autoren als administrativer Mangel angesehen worden (Pigou, 1928 , S. 123, Kaldor, 1955, S. 12, Kelley, 1970, S. 244, Seidel, 1989, S. 29, u.a.). Dabei wird jedoch übersehen, daß der Fiskus das konsumierbare Vermögen in späteren Perioden aufgrund der früheren Angaben des Stpfl. kalkulieren kann. Der Marktzins $r$ ist auch dem Fiskus bekannt. Hat der Stpfl. in der Vergangenheit eine Ersparnis $S+F$ deklariert, so muß er heute den Betrag $(1+r)(S+F)$ ausgezahlt erhalten, den er konsumptiv verwenden wird. Die Finanzbehörde achtet mit anderen Worten darauf, ob die Steuererklärungen der zwei Perioden konsistent sind. Damit ist

$$
C_{2}=w_{2}+(1+r) S-T\left(w_{2}+(1+r)(S+F)\right)
$$

Was geschieht, wenn ein Stpfl. in Periode 2 inkonsistente Angaben macht? In diesem Fall werden Sparen und Entsparen steuerlich nicht anerkannt: Die Konsumsteuern für die zwei Perioden werden allein auf der Basis der Einkünfte $w_{i}$ festgesetzt. Für Periode 1 kann das eine Nachforderung von Steuern beinhalten, die - so sei angenommen - zum Marktzins zu verzinsen ist. Da es, wie unten gezeigt wird, nicht von Nachteil für den Stpfl. ist, daß seine Angaben steuerlich anerkannt werden, achtet er selbst auf die Konsistenz seiner Erklärungen.

Die Bedingungen erster Ordnung für die Aufgabe

$$
\max _{S, F} U\left(C_{1}, C_{2}\right)
$$

unter den Beschränkungen (5.8) und (5.9) sind

$$
\begin{aligned}
& U_{S}=-U_{1}\left(1-T^{\prime}\left(B_{1}\right)\right)+U_{2}(1+r)\left(1-T^{\prime}\left(B_{2}\right)\right)=0 \\
& U_{F}=U_{1} T^{\prime}\left(B_{1}\right)-U_{2}(1+r) T^{\prime}\left(B_{2}\right)=0
\end{aligned}
$$


Dabei bezeichnet $U_{x}$ die erste Ableitung nach dem $x$-ten Argument von $U\left(C_{1}, C_{2}\right)$. Die zwei Bedingungen lassen sich vereinfachen. Teilt man (5.10) durch (5.11), ergibt sich

$$
\begin{aligned}
\frac{1-T^{\prime}\left(B_{1}\right)}{T^{\prime}\left(B_{1}\right)} & =\frac{1-T^{\prime}\left(B_{2}\right)}{T^{\prime}\left(B_{2}\right)} \\
\Leftrightarrow \quad T^{\prime}\left(B_{2}\right) & =T^{\prime}\left(B_{1}\right)
\end{aligned}
$$

Der Grenzsteuersatz ist in jeder Periode gleich hoch. Mit (5.12) wird (5.10) zu

$$
\frac{U_{1}}{U_{2}}=(1+r)
$$

Die Grenzrate der Substitution ist gleich dem intertemporalen Preisverhältnis vor Steuer.

Ist garantiert, daß die Entscheidungen des Stpfl. zu einem positiven Konsum in jeder Periode führen? Da der Konsum in jeder Periode wesentlich ist, gilt

$$
\frac{\partial U}{\partial S}_{\mid C_{1}=0}=\left.\frac{\partial U}{\partial F}\right|_{\mid C_{2}=0}=-\infty
$$

und

$$
\frac{\partial U}{\partial S}_{\mid C_{2}=0}=\frac{\partial U}{\partial F}{ }_{\mid C_{1}=0}=\infty
$$

Der Haushalt wird es daher vermeiden, in einem Umfang zu sparen oder Ersparnisse zu deklarieren, die zu einem Konsum von null in einer Periode führen. Insbesondere ist ausgeschlossen, daß der Stpfl. in Periode 1 keine Steuern zahlt, sich einen hohen Konsum leistet und in Periode 2 weder die ausstehenden Steuern zahlen noch einen Konsum $C_{2}>0$ bestreiten kann.

Im folgenden werden die Optimalbedingungen (5.12) und (5.13) interpretiert. Dabei werden die zwei Fälle eines linearen und direkt progressiven Tarifs unterschieden.

\section{Linearer Tarif}

Mit konstantem Grenzsteuersatz $t$ ist (5.12) unabhängig von der Höhe der deklarierten Ersparnis $S+F$ immer erfüllt. Bei gegebener tatsächlicher Ersparnis $S$ erhöht $F$ den Konsum $C_{1}$ um $t F$ (die Steuerschuld sinkt) und vermindert $C_{2}$ um $(1+r) t F$ (die Steuerschuld steigt). Der Haushalt nimmt einen Kredit bei dem Staat auf, den er zum Marktzins $r$ verzinsen muß. Das kann er aber genauso gut auch bei seiner Bank erreichen. Die fiktiven Ersparnisse haben keinen Einfluß auf 
die intertemporale Budgetgerade nach Steuer. Aus (5.8) und (5.9) erhält man mit dem Tarif $T=t B_{i}{ }^{8}$ :

$$
C_{2}=\left(w_{1}(1-t)-C_{1}\right)(1+r)+w_{2}(1-t)
$$

Wir sehen damit: Auch wenn dem Fiskus die Einsicht in die Konten verwehrt ist, führt die Konsumsteuer (auf steuerinklusiver Basis) bei linearem Tarif zu der gleichen Budgetbeschränkung (und dem gleichen Konsum) wie eine Steuer auf alle Nicht-Zinseinkünfte mit gleichem Tarif, wenn

- ein vollkommener Kapitalmarkt existiert,

- es keine Unsicherheitsquelle gibt,

- der Grenzsteuersatz konstant ist,

- der Haushalt alle Ressourcen für den Konsum nutzt,

- und der Konsum in jeder Periode wesentlich ist.

Diese Bedingungen werden (bis auf die letzte) von Graetz (1982, S. 172) für die Äquivalenz einer persönlichen Ausgabensteuer und einer Steuer auf Löhne angegeben ${ }^{9}$ (siehe auch Atkinson/Stiglitz, 1980, S. 70). Die ausschließliche Besteuerung aller Einkommen, die keine Zinsen sind, kommt wiederum einer Einkommensteuer recht nahe, bei der die Zinsen in großem Umfang hinterzogen werden, eine Entdeckung nur mit einer geringen Wahrscheinlichkeit stattfindet, und bei der keine Quellensteuer auf Zinsen erhoben wird. Es stellt sich daher die Frage, ob der Übergang zu einer Konsumsteuer nicht praktisch schon geschehen ist. Die Antwort müßte "ja” lauten, wenn all diese Voraussetzungen zuträfen.

\section{Direkt progressiver Tarif}

Obwohl zuweilen empfohlen wird, die direkte Konsumsteuer mit einem linearen Tarif auszustatten (Mitschke, 1980), scheint die direkte Progression bei der Besteuerung von Haushalten - auf welcher Bemessungsgrundlage auch immer - zumindest in der Wirtschaftspolitik unumstritten zu sein ${ }^{10}$. In der Bundesrepublik hat die Finanzpolitik im Rahmen der Steuerreform 1990 zwar einen Tarif mit linear ansteigendem Grenzsteuersatz zugestanden ("linear progressiver Tarif"). Der

\footnotetext{
${ }^{8}$ Ein Steuerfreibetrag und eine SteuermäBigung werden vernachlässigt.

${ }^{9} \mathrm{Graetz}$ faBt $w_{i}$ als Lohneinkommen auf und nimmt einen vollständig informierten Fiskus an.

${ }^{10}$ Die direkte Progression wird mit verteilungspolitischen Zielen begründet. Effizienzüberlegungen können auch zu einem (im oberen Bereich) regressiven Tarif führen (siehe Wiegard, 1987, S. 120 - 122).
} 
Übergang zu einer Steuerbetragsfunktion bei der - abgesehen von der Eingangsstufe und der oberen Proportionalzone - schon die zweite Ableitung null beträgt, ist jedoch nicht zu erwarten.

Aus (5.12) folgt wegen der direkten Progression

$$
\begin{aligned}
B_{1} & =B_{2} \\
\Leftrightarrow \quad w_{1}-S^{*}-F^{*} & =w_{2}+(1+r)\left(S^{*}+F^{*}\right)
\end{aligned}
$$

Für gegebene $w_{i}$ und jede Ersparnis $S$ erreicht der Stpfl. durch die Angabe fiktiver Ersparnisse $F$, daß seine Steuerbemessungsgrundlage in jeder Periode gleich hoch ist. Löst man die letzte Gleichung nach $S^{*}+F^{*}$ auf, ergibt sich

$$
S^{*}+F^{*}=\frac{w_{1}-w_{2}}{2+r}
$$

Man sieht hier: Ist $w_{2}$ größer als $w_{1}$, so ist die deklarierte Ersparnis negativ, selbst wenn die tatsächliche Ersparnis positiv ist. Der Stpfl. zahlt in Periode $1 \mathrm{mehr}$ Steuern, als er müßte. Wir setzen den Ausdruck für $S^{*}+F^{*}$ in $B_{1}$ und $B_{2}$ ein und erhalten

$$
\begin{aligned}
B^{*}=B_{1}=B_{2} & =\frac{w_{1}(2+r)-\left(w_{1}-w_{2}\right)}{2+r} \\
& =\frac{w_{1}(1+r)+w_{2}}{2+r}
\end{aligned}
$$

Die in jeder Periode gleich hohe Bemessungsgrundlage sei mit $B^{*}$ bezeichnet. Sie ist unabhängig von dem Konsum in den einzelnen Perioden (bzw. der Ersparnis) und abhängig von dem Lebenseinkommen $w_{1}(1+r)+w_{2}$. Zusammen mit (5.9) und (5.8) erhalten wir jetzt die intertemporale Budgetgerade

$$
\begin{aligned}
C_{2} & =w_{2}+(1+r)\left(w_{1}-C_{1}-T\left(B^{*}\right)\right)-T\left(B^{*}\right) \\
& =w_{2}+(1+r)\left(w_{1}-C_{1}\right)-(2+r) T\left(\frac{w_{1}(1+r)+w_{2}}{2+r}\right)
\end{aligned}
$$

Die Wahl des Haushalts zwischen dem Konsum in den zwei Perioden ist nun dadurch charakterisiert, daß die Grenzrate der Substitution $U_{1} / U_{2}$ gleich der Steigung $-d C_{2} / d C_{1}=(1+r)$ dieser Budgetgeraden ist (siehe Bedingung (5.13)). Das Optimierungsproblem des Stpfl. läßt sich in zwei Stufen zerlegen: Zunächst maximiert er $C_{2}$ bei gegebenem $C_{1}$ durch die Wahl von $S$ und $F$. Ergebnis ist die Bedingung (5.12) und damit die Budgetgerade (5.14). Unter der Beschränkung (5.14) wird anschließend $U\left(C_{1}, C_{2}\right)$ maximiert.

Betrachten wir den Term

$$
(2+r) T\left(\frac{w_{1}(1+r)+w_{2}}{2+r}\right)
$$


etwas näher. Es handelt sich um den Wert der Steuerzahlungen bezogen auf Periode 2. Basis des Tarifs $(2+r) T(\cdot /(2+r))$ ist das Lebenseinkommen ausgedrückt in Einheiten des Konsums in Periode 2. An dieser Stelle ist eine Definition des Begriffs Lebenseinkommen angebracht: Das Lebenseinkommen eines Individuums ist seine Kaufkraft gemessen in Konsumausgaben einer bestimmten Periode. In dem Zwei-Perioden-Modell können wir das Lebenseinkommen in Einheiten von $C_{1}$ oder in Einheiten von $C_{2}$ ausdrücken. Für $C_{2}$ kann der Haushalt (vor Steuer) maximal $L_{2}=w_{1}(1+r)+w_{2}$ ausgeben, von $C_{1}$ kann er sich höchstens $L_{1}=w_{1}+w_{2} /(1+r)$ leisten. Ohne direkte Besteuerung ist der "Lebenskonsum" - der Wert der konsumierten Güter bezogen auf einen bestimmten Zeitpunkt - gleich dem Lebenseinkommen:

$$
C_{1}(1+r)+C_{2}=w_{1}(1+r)+w_{2}
$$

Unter der Ausgabensteuer mit einem steuerinklusiven progressiven Tarif, dem Bankgeheimnis und einem Fiskus, der das für den Konsum zur Verfügung stehende Vermögen des Stpfl. aus den deklarierten Ersparnissen ableitet, sind die Konsumausgaben gleich dem Lebenseinkommen abzüglich der Steuer auf Basis des Lebenseinkommens, wie sich aus (5.14) unmittelbar ergibt:

$$
C_{1}(1+r)+C_{2}=w_{1}(1+r)+w_{2}-(2+r) T\left(\frac{w_{1}(1+r)+w_{2}}{2+r}\right)
$$

$\mathrm{Zu}$ dieser Gleichung sollen noch zwei kurze Bemerkungen festgehalten werden: (i) Man kann den Konsum, die Einkommen (exklusive der Zinsen) und die Steuern natürlich auch auf Periode 1 beziehen, indem man beide Seiten der Gleichung durch $1+r$ teilt. (ii) Die Lebenseinkommensteuer belastet eine Basis inklusive der Steuer selbst. Hätten wir den deklarierten Konsum in jeder Periode belastet, hätte sich eine Lebensausgabensteuer auf der Basis $C_{1}(1+r)+C_{2}$ ergeben, da der Teil von $w_{1}$, den der Stpfl. laut eigenen Angaben in Periode 1 nicht konsumiert, in der folgenden Periode - und zwar verzinst - den steuerpflichtigen Konsum erhöht.

In Abbildung 5.2 wird die Konsequenz eines Schutzes der Konten vor dem Fiskus graphisch dargestellt. Rechts in der Abbildung finden wir die Budgetgerade vor Steuer, die linke Gerade ist Gleichung (5.14). Die konkave Budgetbeschränkung ergibt sich, wie in Abschnitt 5.3.1 gezeigt wurde, bei vollständigem Einblick des Fiskus in die Sparkonten. Wählt der Stpfl. in jeder Periode den gleichen Konsum, profitiert er nicht von dem Bankgeheimnis. Bei ungleichmäßigem Konsumstrom hat er gegenüber dem Zustand ohne Bankgeheimnis einen Vorteil. In der Abbildung kann er bei gleichem Konsum $\tilde{C}_{1}$ unter beiden Regimen unter dem System mit dem Bankgeheimnis den Betrag $A B$ mehr von $C_{2}$ konsumieren. Dieser Vorteil resultiert aus der Angleichung der Steuerbemessungsgrundlagen in den zwei Perioden. Algebraisch beträgt der Vorteil des interperiodischen Progressionsausgleichs 
Abbildung 5.2: Progressionsausgleich unter einer Konsumsteuer

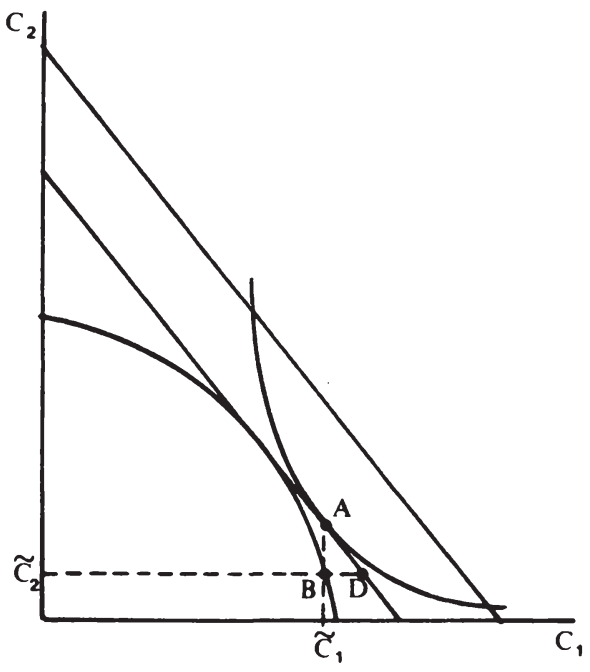

(siehe Anhang 5.3.2):

$$
V I P_{2}=(1+r) T\left(w_{1}-\tilde{S}\right)+T\left(w_{2}+(1+r) \tilde{S}\right)-(2+r) T\left(\frac{w_{1}(1+r)+w_{2}}{2+r}\right)
$$

Dabei ist $\tilde{S}$ die Ersparnis, die sich bei einem Konsum $\tilde{C}$ und der Besteuerung auf der Basis des tatsächlichen Periodenkonsums (inklusive Steuer) ergibt. Mit $w_{1}-\tilde{S} \neq w_{2}+(1+r) \tilde{S}$ ist $V I P_{2}$ positiv.

An dieser Stelle kann die Behauptung überprüft werden, daß der Stpfl. konsistente Angaben bezüglich seiner Ersparnisse machen wird. Inkonsistente Angaben führen dazu, daß deklarierte Ersparnisse und Entsparen steuerlich nicht anerkannt werden. Die endgültige Steuerlast beider Perioden zusammen beträgt damit (bezogen auf Periode 2) $(1+r) T\left(w_{1}\right)+T\left(w_{2}\right)$. Nur in dem Fall $w_{1}=w_{2}$ ist diese Last gleich der Lebenseinkommensteuer bei konsistenten Angaben; mit $w_{1} \neq w_{2}$ vergrößert sich die Steuerbelastung durch inkonsistente Angaben, da die Grenzsteuersätze in den zwei Perioden dann nicht gleich groß sind.

Die Durchschnittsbildung der Steuerbemessungsgrundlagen ("averaging") einer periodischen Ausgabensteuer wird in der Literatur als wünschenswert angesehen (Kaldor, 1965, S. 52, Bradford, 1986, S. 91). Sie wird in dem Modell dieses Abschnitts durch die Stpfl. selbst bewerkstelligt. Unter der Annahme eines sicheren Zinssatzes und sicherer Einkommenserwartungen ist es in dem Modell nicht erforderlich, daß der Fiskus die Durchschnittsbildung durchführt. Trotz des "averaging" 
Abbildung 5.3: Benachteiligung der Haushalte mit ungleichmäßigem Einkommen unter einer progressiven Lohnsteuer

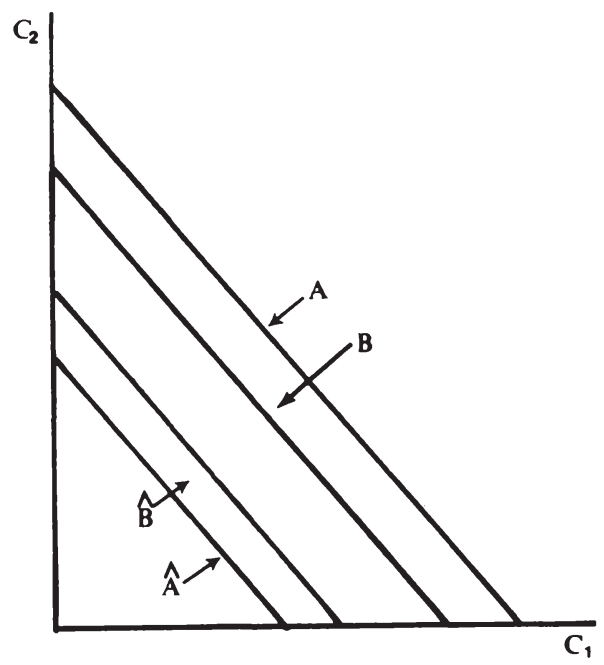

geht die Progression nicht verloren: Haushalte mit einem höheren Lebenseinkommen müssen sich im $C_{1}-C_{2}$-Diagramm eine größere Parallelverschiebung ihrer Budgetgeraden gefallen lassen, als Haushalte mit einem geringeren Lebenseinkommen. Ihre Budgetgerade nach Steuer bleibt jedoch rechts von der Beschränkung nach Steuer der weniger Begüterten: Bei konstantem $r$ ergibt die Differentiation von Gleichung (5.15)

$$
d\left(C_{1}(1+r)+C_{2}\right)=\left(1-T^{\prime}\left(\frac{L_{2}}{2+r}\right)\right) d L_{2}>0 .
$$

Einem Stpfl. mit einem geringeren $L_{2}$ verbleibt mehr von einem Lebenseinkommenszuwachs. Dies ist mit einer progressiven periodischen Steuer auf der Basis der Lohneinkommen bzw. der $w_{i}$ - diese Steuer kann man als degenerierte Einkommensteuer auffassen, bei der alle Zinsen hinterzogen werden und keine Kontrollen stattfinden - nicht der Fall. Die Budgetgerade ist mit dieser Steuer (der Tarif sei wieder mit $T$ bezeichnet)

$$
C_{2}=w_{2}+(1+r)\left(w_{1}-C_{1}-T\left(w_{1}\right)\right)-T\left(w_{2}\right)
$$

Die Budgetbeschränkungen von Haushalten mit einem gleich hohen Lebenseinkommen stimmen nur dann überein, wenn seine Zusammensetzung gleich ist. Gleiche, 
gemessen an der Höhe des Lebenseinkommens, werden ungleich behandelt. Andererseits kann die in Abbildung 5.3 dargestellte Situation eintreten. Individuum $A$ ist vor Steuer reicher als $B$. Nach Steuer ist $B$ besser gestellt (er hat die Konsummöglichkeiten $\widehat{B}$ ) als $A$ (mit den Möglichkeiten $\widehat{A}$ ), wenn Haushalt $A$ im Vergleich zu $B$ über ein genügend ungleichmäßig anfallendes Einkommen $w_{i}, i=1,2$, verfügt. Der Beleg für diese Möglichkeit ist schnell erbracht: Bei gegebenem $C_{1}$ und mit

$$
d L_{2}=(1+r) d w_{1}+d w_{2}=0
$$

folgt aus (5.16) $d C_{2}=\left(T^{\prime}\left(w_{1}\right)-T^{\prime}\left(w_{2}\right)\right) d w_{2}$. Der Konsum $C_{2}$ sinkt, wenn $w_{2}>w_{1}$ ist und wenn $w_{2}$ auf Kosten von $w_{1}$ wächst, oder wenn $w_{2}<w_{1}$ ist und $w_{2}$ kleiner wird.

\subsubsection{Zwei alternative Verfahren des Progressionsausgleichs}

Der oben dargestellten Methode des Progressionsausgleichs sollen jetzt die zwei Verfahren gegenübergestellt werden, die einerseits von Meade (1978) und Bradford (1984), andererseits von Pollak (1989) vorgeschlagen worden sind. Der Vorschlag von Pollak geht davon aus, daß der Fiskus Einkommen, Ersparnisse und Konsum des Stpfl. kennt. Er kann daher für einen Progressionsausgleich zwischen verschiedenen Perioden sorgen. In dem Zwei-Perioden-Modell zahlt der Haushalt in Periode 1 die Steuer $K\left(C_{1}\right)^{11}$. In Periode 2 hat er eine Verpflichtung in Höhe von

$$
((1+r)+1) K\left(\frac{C_{1}(1+r)+C_{2}}{(1+r)+1}\right)-(1+r) K\left(C_{1}\right)
$$

Die Konsumsteuer wird auf der Basis des kumulierten Wertes der Konsumausgaben in den zwei Perioden berechnet. Die in Periode 1 gezahlte Steuer wird aufgezinst und angerechnet. Die endgültige Steuerschuld hängt damit nur von der Höhe des aufgezinsten Konsumstroms, nicht aber von dessen Zusammensetzung ab 12. Dieses amtliche Ausgleichsverfahren hat zwei Vorzüge (Pollak, 1989, S. 23): (i) Mit $C_{1}=C_{2}$ findet kein amtlicher Progressionsausgleich statt. D.h. in jedem Jahr entsteht die gleiche Steuerlast $K(C)$. (ii) Man benötigt nicht je nach der Anzahl

\footnotetext{
${ }^{11}$ Alternativ könnte natürlich auch der belastungsgleiche steuerinklusive Tarif $T(\cdot)$ verwendet werden.
}

${ }^{12}$ Die Steuerschuld $Z_{i}$ in Periode i beträgt bei mehr als zwei Perioden:

$$
Z_{i}=\delta_{i} K\left(\frac{1}{\delta_{i}} \sum_{j=1}^{i} C_{j}(1+r)^{j-1}\right)-(1+r) Z_{i-1}, \quad \delta_{i}=\sum_{j=1}^{i}(1+r)^{j-1}
$$


der Perioden, über die der Konsum kumuliert wird, einen anderen Tarif. Es sind lediglich die Funktionen $K(\cdot)$ und der kumulierte Wert des Konsums mit einem Diskontfaktor zu multiplizieren bzw. zu dividieren, der von dem Kumulationszeitraum und dem Zins abhängt. Beide Eigenschaften sind mit anderen Vorschlägen zu einer kumulativen Durchschnittsbesteuerung nicht unbedingt verbunden ${ }^{13}$.

Meade (1978) und Bradford $(1984,1986)$ gehen im Grunde davon aus, daß der Fiskus nicht die gesamten Ersparnisse eines Haushalts feststellen kann. Nur die Anlagen bei Pensionskassen, inländischen (buchführungspflichtigen) Firmen und Finanzintermediären kann er eventuell in Erfahrung bringen (Bradford, 1986, S. 90). Meade und Bradford teilen die Ersparnis daher in zwei Kategorien auf: Ersparnisse in registrierten Anlagen ("registered assets" bei Meade und "qualified accounts" bei Bradford) sind steuerlich abzugsfähig, Entsparen aus registrierten Anlagen ist auf der anderen Seite steuerpflichtig. Sparen und Entsparen in vom Fiskus nicht registrierten bzw. nicht kontrollierbaren Anlagen ("unregistered assets", "unqualified accounts") - Auslandsanlagen zum Beispiel - werden steuerlich nicht berücksichtigt (Meade, 1978, S. 175 - 183, Bradford, 1984, S. 107 - 115).

Bezeichnen wir die registrierten und nicht registrierten Ersparnisse mit $S_{r}$ und $S_{u}$, ergeben sich für das Zwei-Perioden-Modell die Beschränkungen

$$
\begin{aligned}
& C_{1}=w_{1}-S_{r}-S_{u}-T\left(w_{1}-S_{r}\right) \\
& C_{2}=w_{2}+(1+r)\left(S_{r}+S_{u}\right)-T\left(w_{2}+(1+r) S_{r}\right)
\end{aligned}
$$

Dabei ist unterstellt, daß beide Anlagen vor Steuer dieselbe Verzinsung abwerfen. Wie wird ein rationaler Stpfl. seine Ersparnisse zwischen registrierten und nicht registrierten Anlagen aufteilen? Mit Hilfe der Variablen $S_{r}$ wird er die Grenzsteuersätze in den beiden Perioden angleichen. Mit den Ersparnissen $S_{u}$ kann er dann zu der Nettotransformationsrate $1+r$ seine Konsummöglichkeiten auf die zwei Perioden aufteilen. Das Verfahren von Meade und Bradford überläßt es also wieder den Haushalten, einen Progressionsausgleich vorzunehmen.

Es sind drei Verfahren einer interperiodischen Durchschnittsbesteuerung dargestellt worden. Alle drei Verfahren kommen zu dem gleichen Ergebnis: Steuerlich belastet wird letztlich das Lebenseinkommen bzw. der Lebenskonsum ${ }^{14}$. Sie unterscheiden sich jedoch durch einen unterschiedlich hohen Informationsbedarf des Fiskus über die Sparkonten. Bei Pollak muß er die gesamten Ersparnisse kennen, um den Komsum (indirekt) feststellen zu können. Bei Meade und Bradford

\footnotetext{
${ }^{13}$ Der Tarif von Mitschke (1980, S. 133 f.) z.B. erfüllt (i) nicht.

${ }^{14}$ Alle drei Verfahren haben eine interessante Nebenwirkung. Betrachten wir zwei Individuen A und B mit gleichem Lebenseinkommen vor Steuer. A habe eine längere Lebenszeit als B. Man kann leicht zeigen, daB die Lebenseinkommensteuer von A geringer ist als die von B. Das längere Leben wird also steuerlich belohnt. Ob dieser Effekt wünschenswert ist, soll hier nicht diskutiert werden. Er würde im Alter eine kleine Versicherung gegen ein langes Leben darstellen.
} 
kennt der Fiskus nur die Ersparnisse in registrierten Anlagen. Das in diesem Kapitel dargestellte Verfahren kann mit einem Schutz der Konten vor dem Einblick der Steuerfahndung leben, solange nur die Quellen der Ersparnisse - alle NichtZinseinkommen - feststellbar sind. Wenn die Bürger - aus welchen Motiven auch immer - eine ausgeprägte Präferenz für das Bankgeheimnis haben, im übrigen aber mit Steuerprüfungen leben können, bietet sich die zuletzt genannte Methode an. Bevor dieses Angebot jedoch in Erwägung gezogen werden könnte, ist im nächsten Abschnitt zu prüfen, ob das Verfahren auch unter weniger einfachen Modellannahmen, als bisher verwendet wurden, Bestand haben kann.

\subsection{Verzicht auf die Einsichtnahme in Sparkonten?}

Der Vorschlag, der Fiskus solle auf eine Einsichtnahme in die Sparkonten verzichten, weckt Zweifel, sobald man über die Grenzen des in Abschnitt 5.3 dargestellten Modells blickt. Im folgenden wird daher geprüft, ob der Vorschlag unter wirklichkeitsnäheren Voraussetzungen nicht verworfen werden muß. Insbesondere wird gefragt, welche Konsequenzen der Schutz der Bankkunden vor dem Fiskus hat, wenn

- bestimmte Parameter Zufallsvariable sind;

- es ein für den Fiskus nicht zugängliches Ausland gibt, in das ein Stpfl. seine Ersparnisse exportieren kann.

Anschließend wird ein Vorschlag zur Administration einer Konsumsteuer skizziert, der diese Komplikationen berücksichtigt. Zuerst soll jedoch kurz auf die in der Literatur enthaltenen Vorschläge zur Erhebung einer Konsumsteuer eingegangen werden. Dabei wird gezeigt, daß diese einen zentralen Mangel haben: Sie beruhen auf der den Bürgern so unliebsamen Offenlegung der Konten gegenüber dem Staat.

\subsubsection{Kontrollmitteilungen und Quellenabzug zur Erhebung einer Konsumsteuer}

Kaldor wollte die Ausgabensteuer mit folgendem System von Kontrollmitteilungen verbunden wissen: Der Verkäufer einer Finanzanlage bestätigt dem Käufer schriftlich alle steuerlich relevanten Daten der Transaktion. Der Käufer unterschreibt ebenfalls und schickt das Formular an die Finanzverwaltung, die mit Hilfe dieser Daten die in den Steuererklärungen deklarierten Finanztransaktionen überprüft (Kaldor 1955, S. 217, 1964, S. 98). Abweichungen zwischen Steuererklärung und 
Kontrollmitteilung soll nachgegangen werden; zusätzlich werden die Angaben der Finanzintermediäre routinemäßig bei Betriebsprüfungen kontrolliert. Ein anderer Vorschlag zur Steuersicherung unter einer persönlichen Ausgabensteuer stammt von Mitschke (1986, S. 175 f.): Auf Zinserträge werden wie auf Lohneinkommen Quellensteuern erhoben, die bei Vorlage von Depotauszügen und Lohnsteuerkarte auf die jährliche Konsumsteuerschuld angerechnet werden.

Der Vorschlag von Kaldor scheint zumindest in einer geschlossenen Volkswirtschaft funktionsfähig zu sein. Der Vorschlag von Mitschke weckt dagegen Zweifel, die kurz ausgeführt werden sollen. Wenn ein Stpfl. ein Wertpapier verkauft und den Erlös samt Zinsen für Konsumausgaben verwendet, stellt eine Quellensteuer allein auf Zinsen - selbst mit einem Satz von $100 \%$ - nur eine geringe Vorauszahlung auf die anfallende Konsumsteuerschuld dar. Der Hauptzweck der Maßnahme ist auch eher, die Haushalte zu veranlassen, ihre Depotauszüge, auf denen die gezahlte Quellensteuer bestätigt wäre, im Rahmen der Konsumsteuerveranlagung vorzulegen. Wie Kapitel 4 gezeigt hat, ist aber zumindest nicht offensichtlich, ob ein Stpfl. nicht auf die Anrechnung von Quellensteuern verzichten wird. Um die Konsumsteuer mit Hilfe von Abzügen an der Quelle durchzusetzen, wäre es besser, eine Quellensteuer auf die Verkäufe und Käufe von Finanzaktiva zu erheben: Käufe werden mit einem Satz $b$ subventioniert, Verkäufe (inklusive Zinsgutschrift) werden mit dem Satz $b$ belastet. Wäre der (steuerinklusive) Tarif der Konsumsteuer linear mit dem Grenzsteuersatz $b$ und würden auf Löhne und übrige Einkünfte ebenfalls eine Quellensteuer mit dem Satz $b$ erhoben, können die Haushalte die Konsumsteuer nicht hinterziehen. Bei progressivem Tarif der Ausgabensteuer ergeben sich dagegen wieder Probleme, da Vorwegabzug und endgültige Steuerschuld nicht übereinstimmen werden.

Die in der Literatur enthaltenen Vorschläge zur Erhebung einer Konsumsteuer Kontrollmitteilungen und Quellenabzug - scheinen brauchbar zu sein. Ihr Nachteil ist nur, daß sie ein Bankgeheimnis nicht zulassen können.

\subsubsection{Unsicherheit}

In dem Rahmen des Zwei-Perioden-Modells aus Abschnitt 5.3.2 sollen hintereinander drei Unsicherheitsquellen diskutiert werden: Wenn der Stpfl. in Periode 1 (bei gegebenem $w_{1}$ ) über seine tatsächlichen und seine deklarierten Ersparnisse entscheidet, kann unsicher sein,

- welchen Wert das exogene Einkommen $\widetilde{w}_{2}$ in Periode 2 annimmt;

- welche Verzinsung vor Steuer seine Ersparnisse abwerfen;

- ob der Stpfl. in Periode 2 noch lebt. 
Bei unsicheren Einkommenserwartungen ( $\tilde{w}_{2}$ ist Zufallsvariable) sind die Grenzsteuersätze nach Realisation von $\tilde{w}_{2}$ nur zufällig gleich hoch. Lediglich ex ante stellt sich ein gewisser Progressionsausgleich ein. Bei risikoneutralen Haushalten ergibt sich bezüglich der deklarierten Ersparnisse die Optimumbedingung $T^{\prime}\left(B_{1}\right)=$ $E\left(T^{\prime}\left(B_{2}\right)\right)$, wobei $E$ der Erwartungswertoperator ist. Bei risikoaversen Haushalten sind die Grenzsteuersätze im Erwartungswert nicht genau gleich. Ex post gelingt es dem Stpfl. unabhängig von seiner Risikoeinstellung nicht, seine Steuerbelastung zu minimieren. Wenn man von dem Grundsatz ausgeht, daß "people should be taxed on their actual, not their expected consumption" (Graetz, 1980, S. 172), gibt es jedoch eine einfache Lösung. Auf Antrag des Stpfl. führt der Fiskus einen interperiodischen Progressionsausgleich durch. Nach Realisation von $\tilde{w}_{2}$ berechnet er die Steuer

$$
(2+r) T\left(\frac{w_{1}(1+r)+w_{2}}{2+r}\right)
$$

wobei $w_{2}$ der Wert ist, den $\tilde{w}_{2}$ angenommen hat. Davon abgezogen wird die (verzinste) Steuer, die in Periode 1 entrichtet worden ist. Das Verfahren entspricht dem oben dargestellten von Pollak mit dem Unterschied, daß in Periode 1 nicht die tatsächlichen Konsumausgaben Steuerbasis sind.

Ex ante können also die Haushalte einen gewissen Progressionsausgleich planen, ex post würde ein amtliches Ausgleichsverfahren dafür sorgen, daß das realisierte Lebenseinkommen belastet wird. Auf ein ex ante-averaging könnte in einem einfachen Modell natürlich verzichtet werden, wenn es keinen Einfluß auf die intertemporale Budgetbeschränkung hat. In praxi ist es jedoch wünschenswert, da sowohl die Haushalte als auch der Fiskus vorziehen werden, über die Zeit relativ gleichmäßig verteilte Steuerzahlungen zu leisten bzw. zu erhalten. Ein ex postaveraging ist notwendig, wenn die Besteuerung letztlich auf realisierten Größen beruhen soll. Zudem wird es Stpfl. geben, die nicht beständig ihre erwartete Steuerlast minimieren und lieber ex post einen Progressionsausgleich in Anspruch nehmen.

Eine zweite mögliche Unsicherheitsquelle in dem Entscheidungsproblem des Stpfl. ist die Verzinsung seiner Ersparnis. In der Praxis gibt es ja nicht eine einzige sichere Anlage, sondern eine Vielzahl von Anlagen mit ganz unterschiedlichen Chancen und Risiken. Wenn der Fiskus die genaue Höhe und Zusammensetzung der Ersparnisse eines Haushalts nicht in Erfahrung bringen kann, bleibt nur ein Ausweg, um "die" Verzinsung der von den Haushalten deklarierten Ersparnisse festzustellen: Es wird für Zwecke der Besteuerung eine Durchschnittsrendite festverzinslicher Wertpapiere ermittelt, mit deren Hilfe das konsumierbare Vermögen eines Haushalts kalkuliert wird, der seine deklarierten Ersparnisse nicht belegen will. Speziell bietet sich die durchschnittliche Rendite der Staatsschuldtitel an, da sie infolge der Größe des entsprechenden Kapitalmarktsegments eine stabile 
Kursentwicklung aufweisen. Diese Pauschalierung mag rigoros erscheinen. Sie ist jedoch der Preis, der für das Bankgeheimnis zu zahlen wäre. Der Preis ist so hoch nicht, wenn man überlegt, wie verschiedene Gruppen von Sparern von der Pauschalierung betroffen werden.

Viele renditebewußte und risikoscheue Haushalte halten einen Großteil ihres Geldvermögens in Form festverzinslicher Wertpapiere. Die Durchschnittsrendite der Staatsschuldtitel dürfte für die Rendite ihres Portfolios eine gute Näherung sein. Viele Sparer greifen freilich auch auf andere Anlagen zu: niedrig verzinste mit hoher Liquidität und ohne Risiko (bis auf das Inflationsrisiko) und auf der anderen Seite höher verzinsliche Anlagen mit hohen Risiken (Aktien und Auslandsanlagen mit einem Wechselkursrisiko etwa). Auf Sparkonten mit gesetzlicher Kündigungsfrist befinden sich hohe Beträge, obgleich die gebotene Verzinsung erheblich niedriger ist als die von Bundesobligationen (z.B. 2,8 \% im Vergleich zu ca. 7,8 \%). Die Konsequenz, die deklarierten Ersparnisse steuerlich mit 7,8 \% zu verzinsen, obwohl die tatsächliche Ersparnis nur 2,8 \% erbracht hat, wäre unklug. Zwei einfache Lösungen bieten sich an: (i) Die Sparer können ihr Geld in Staatsschuldtiteln anlegen, statt es zum Vorteil der Banken auf niedrig verzinsten Sparkonten zu lassen. Anleihen des Staates mit geringer Restlaufzeit etwa können ebenfalls ohne großen Verlust liquidiert werden; zusätzliche Gebühren, die die Renditedifferenz schmälern, fallen kaum an, da - um wieder auf bereits bestehende Institutionen zurückzugreifen - die Bundesschuldenverwaltung keine Depotgebühren berechnet. Verteilungspolitisch weniger glücklich wäre die zweite Lösung: (ii) Den Sparern wird die Option gegeben, dem Finanzamt ihre Sparkonten durch Vorlage der Bankbelege zu offenbaren. Unter dieser Bedingung wird steuerlich die tatsächliche Verzinsung der Sparguthaben berücksichtigt.

Eine Reihe von Haushalten investiert in Finanzanlagen, deren Rendite von der Durchschnittsrendite öffentlicher Anleihen erheblich nach oben und unten abweichen kann. Es kann daher dazu kommen, daß sie teilweise steuerfrei konsumieren oder daß sie - bei weniger glücklichen Investitionen - zuviel Konsumsteuern zahlen. Dies wäre für die angesprochenen Haushalte nicht neu, da sie auch unter der bestehenden Einkommensteuer ganz ähnlich behandelt werden: Kapitalgewinne im Privatvermögen sind grundsätzlich steuerfrei. Das zu versteuernde Einkommen ist daher größer (kleiner) als das ökonomische Einkommen, wenn ein Investor überwiegend Kapitalverluste (-gewinne ) zu verbuchen hat ${ }^{15}$. Wenn der Staat jedoch an Erfolg und Mißerfolg der Geldanlagen von Haushalten beteiligt werden soll, kann diesen unter der Konsumsteuer wieder die Möglichkeit gegeben werden,

\footnotetext{
${ }^{15}$ Es soll kurz an die Definition des ökonomischen Jahreseinkommens erinnert werden: Es ist der Betrag, den ein Individuum während eines Jahres konsumieren könnte, ohne das Kapitalvermögen, das er am Anfang des Jahres hat, zu vermindern oder zu erhöhen (Meade, 1978, S. 31).
} 
ihre riskanten Finanzinvestitionen zum Zeitpunkt der Anlage anzuzeigen und ihre spätere Liquidation ebenfalls zu dokumentieren.

Die dritte Unsicherheitsquelle ist der Todeszeitpunkt des Stpfl. Betrachten wir folgenden Fall: Mit der Wahrscheinlichkeit $u>0$ überlebt der Haushalt Periode 1 und stirbt am Ende der Periode 2. Mit der Wahrscheinlichkeit $1-u>0$ stirbt er schon am Ende der Periode 1. Genügen die Präferenzen des Haushalts über Konsum in den zwei Perioden den Axiomen der Erwartungsnutzentheorie, hat er die Nutzenfunktion

$$
u U\left(C_{1}, C_{2}\right)+(1-u) U\left(C_{1}, 0\right)
$$

Es liegt nun der Verdacht nahe, daß ein Stpfl. mit einer geringen Überlebenswahrscheinlichkeit $u$ in Periode 1 nur sehr wenig spart, aber eine hohe Ersparnis deklariert, um noch einen hohen sicheren Konsum $C_{1}$ zu genießen. Wenn er tatsächlich stirbt, wäre es ihm gelungen, endgültig und nicht nur temporär Steuern zu verkürzen. Ein exogenes Einkommen $w_{2}$, aus dem der Staat seine Ansprüche befriedigen könnte, soll nicht anfallen, wenn der Stpfl. verschieden ist. Der Verzicht auf die Konteneinsicht würde damit diesen Stpfl. im Vergleich zu einem anderen, der eine höhere Überlebenswahrscheinlichkeit hat (zum Beispiel $u=1$ ), bevorzugen.

Der ausgesprochene Verdacht ist jedoch nicht richtig. Auch ein Stpfl., der das Ende von Periode 1 mit einer Wahrscheinlichkeit $u<1$ überlebt, deklariert Ersparnisse, die (im Fall eines Überlebens) einen Ausgleich der Grenzsteuersätze in den zwei Perioden herbeiführen (siehe Anhang 5.4.2). Nur die Höhe der tatsächlichen Ersparnis wird durch den Wert von $u$ beeinflußt. Es kann daher nicht gesagt werden, daß zwei Haushalte mit unterschiedlichen Überlebenswahrscheinlichkeiten, die im übrigen über gleiche Konsummöglichkeiten vor Steuer verfügen, infolge des Bankgeheimnisses steuerlich ungleich behandelt werden.

\subsubsection{Auslandsanlagen}

Gibt es keine Kapitalverkehrsbeschränkungen und existieren Länder, in denen die Steuerbelastung geringer als im Inland ist, ergeben sich für jede Steuer Probleme der Steuerhinterziehung und -umgehung, da die Fiskalhoheit des inländischen Staates an der Grenze endet. Die Literatur vermittelt nun den Eindruck, daß eine Ausgabensteuer in einer offenen Volkswirtschaft auf größere Erhebungsschwierigkeiten stößt als eine Einkommensteuer (z.B. Seidel, 1989, S. 36 f.). Im folgenden soll gezeigt werden, daß unter den gegebenen institutionellen Verhältnissen das Gegenteil richtig sein wird.

Kommen wir direkt zu dem Fall, der immer wieder angeführt wird, um die außenwirtschaftliche Problematik einer direkten Ausgabensteuer zu belegen (vgl. Meade, 1978, S. 419 f., Graetz, 1980, S. 253, Wenger, 1985, S. 311, Seidel, 1989, 
S. 37). Ein Stpfl. arbeitet im Inland, verdient und spart viel während seines Erwerbslebens, zahlt aber wenig Konsumsteuern. Er exportiert seine Ersparnisse und emigriert schließlich in ein Land, in dem er z.B. nur eine geringe Einkommensteuer auf seine künftigen Zinserträge zahlen muß. Der Vorteil, zum Teil steuerfrei konsumieren zu können, wird natürlich noch viel größer, wenn im Inland nicht bloß die tatsächlichen, sondern zusätzlich fiktive Ersparnisse die Konsumsteuerschuld vermindert haben. Um einen Export von Sparkapital samt Sparern in das Ausland zu bremsen, schlagen Lodin (1972, S. 103) und Meade (1978, S. 438 442) eine Emigrationsteuer vor, deren Bemessungsgrundlage das exportierte $\mathrm{Ka}$ pital ist. Diese Steuer soll so bemessen sein, daß sie einer Vorauszahlung auf die Konsumsteuern entspricht, die bei einem Konsum des Kapitals im Inland anfallen würden. In $\S 2$ Außensteuergesetz findet sich zwar eine gesetzliche Grundlage für eine "Fluchtsteuer". Leider ist eine Emigrationsteuer bei freiem internationalen Kapital- und Personenverkehr nicht durchsetzbar (Graetz, 1980, S. 254) Die Stpfl. werden unter diesen Bedingungen schneller als der inländische Fiskus sein. Es gibt wirksamere Maßnahmen gegen emigrationsbereite Stpfl. Die steuerliche abzugsfähige Ersparnis könnte nach oben beschränkt werden: Eine höhere Ersparnis als z.B. $25 \%$ des Einkommens wird nicht anerkannt. Für eine Vielzahl von Stpfl. gibt es eine weniger pauschale Lösung. Rentenbeiträge werden "automatisch" von dem Lohneinkommen abgezogen. Die späteren Rentenansprüche könnten dem Fiskus als Sicherheit dafür dienen, daß keine Konsumsteuern durch eine Auswanderung hinterzogen werden.

Angesichts solcher Maßnahmen bleibt die Frage, ob eine Emigration aus steuerlichen Motiven ein häufig zu erwartender Fall ist. Die Mehrzahl der Stpfl. ist doch eher inlandsgebunden. Ihr Sparkapital exportieren sie allerdings in großen Summen. Diese Verhaltensweise stellt für die Konsumsteuer jedoch kein Problem dar, da die Stpfl. "freiwillig" Ersparnisse deklarieren, wenn der Fiskus die Quellen der Ersparnis kennt. Der Fall, daß ein Stpfl. im Inland bleibt und nur sein Geld im Ausland investiert, stellt die Einkommensteuer im Gegensatz zu der Konsumsteuer jedoch vor Schwierigkeiten: Die Stpfl. behaupten, sie sparen nicht. Der Fiskus kann diese Behauptung nicht nachprüfen, wenn das Kapital in ein Land exportiert wird, das keine Zusammenarbeit mit den Finanzbehörden des Kapitalexportlandes wünscht.

\subsubsection{Die Administration einer Konsumsteuer}

Bevor zusammenfassend die Maßnahmen skizziert werden, die eine direkte Konsumsteuer selbst unter der Nebenbedingung administrierbar erscheinen lassen, daß den Bürgern der Schutz ihrer Konten vor dem Fiskus garantiert wird, soll kurz auf drei weitere Punkte eingegangen werden. Es sind dies die Behandlung von Erb- 
schaften, die Frage einer Erfassung von Vermögen im Zeitpunkt der Einführung einer Konsumsteuer und die Frage, wie ein Bankgeheimnis angesichts des organisierten Verbrechens zu bewerten ist.

Ein Großteil der Ersparnisse bzw. des ererbten Vermögens wird nicht konsumiert, sondern vererbt. Es ist umstritten, wie Erbschaften unter der Konsumsteuer behandelt werden sollen. Man kann sie als Konsum des Erblassers und/oder als Einkünfte des Erben ansehen, die dessen steuerliche Konsummöglichkeiten erhöhen. Man könnte es auch bei der bestehenden Erbschaftsteuer belassen und das resultierende Nettoerbe den Einkünften des Erben zuschlagen. Im Vergleich zu der Behandlung von Erbschaften unter unserer Einkommensteuer spart der Erblasser Steuern, da die Ersparnisse für die Nachkommen seinen Konsum vermindern; der Erbe wird zusätzlich belastet, soweit er das Erbe ausgibt. Welche Gestaltung auch immer gewählt wird: Erhebungsprobleme ergeben sich bezügich inländischer Vermögen kaum, wenn an den $\S \S 33$ und 34 Erbschaftsteuergesetz festgehalten wird. Danach sind Kreditinstitute verpflichtet, dem Finanzamt im Todesfall eines Kunden dessen Vermögen anzuzeigen. Bei einem Export des Vermögens "in ein Gebiet außerhalb des Geltungsbereichs" des ErbStG können sie für die Erbschaftsteuer haftbar gemacht werden ( $§ 20$ Abs. 6 Satz 2 ErbStG). Standesämter, Gerichte und Notare informieren das Finanzamt über Sterbefälle, erteilte Erbscheine und andere Tatsachen, die für die Erbschaftsteuer bedeutsam sind (siehe Bilsdorfer, 1989, S. 54 - 56 und S. 66 - 70 zu Einzelheiten der Mitteilungspflichten von Banken und staatlichen Stellen gegenüber den Finanzämtern). Im Todesfall erlischt also das Bankgeheimnis. Werden die $\S \S 33$ und 34 ErbStG konsequent angewendet, gibt es wenig Spielraum, anläßlich eines Übergangs inländischen Vermögens im Todesfall Steuern zu sparen. Wird das Vermögen zu Lebzeiten des Erblassers ins Ausland verbracht, kann eine Erbschaftsteuer zwar hinterzogen werden. Die vererbten Ersparnisse würden dann aber auch die Konsumsteuern des Erblassers nicht vermindern.

Bislang haben wir die Konsumsteuer immer unter der Prämisse diskutiert, daß sie bereits seit langem in Kraft ist. Der Übergang von einer Einkommen- zu einer Konsumsteuer ist der Literatur zufolge mit besonderen Problemen verbunden (siehe u.a. Lodin, 1972, S. 123 - 127, Bradford, 1984, Kapitel 6). Kommen wir direkt zu dem Punkt, der das Bankgeheimnis betrifft: Meade (1978, S. 176) schreibt, der Regimewechsel "will require the widest degree of registration (of assets (R.H.)) in order to limit opportunities for subsequent expenditure without incurring a charge to tax" (siehe auch Lodin, 1972, S. 123). Ich glaube nicht, daß die Erfassung aller Vermögen notwendig ist. Erstens übersieht das Argument von Meade, daß das Vermögen im Zeitpunkt der Ablösung der Einkommensteuer aus (zum Teil) versteuertem Einkommen stammt. Eine zusätzliche Konsumsteuer bei der Ausgabe dieses Vermögens ist nicht einzusehen. Zweitens ist die Belastung 
des Konsums, der mit (Nicht-Zins-)Einkünften finanziert wird, die nach dem Regimewechsel anfallen, nicht auf die Registrierung des Vermögens im Zeitpunkt des Übergangs angewiesen.

Die Garantie eines Bankgeheimnisses trifft dort auf eine Grenze, wo das organisierte Verbrechen beginnt. Ein Bankgeheimnis kann nur den im Prinzip gesetzestreuen Bürgern garantiert werden. Wer sich dadurch verdächtig macht, daß er große Beträge in bar auf Konten einzahlt und abhebt, sollte damit rechnen müssen, daß die Banken Kontrollmitteilungen an die Polizei und Finanzämter schicken und $\mathrm{daß}$ er die legale Herkunft seines Geldes beweisen muß. Ohne solche Maßnahmen besteht wenig Hoffnung, die Gewinne des organisierten Verbrechens zu beschlagnahmen - was aus ökonomischer Sicht eine notwendige Bedingung ist, um die Ausbreitung dieses Geschäfts zu verhindern.

Ich hoffe, daß die Einwände gegen den Verzicht auf eine Einsichtnahme in private Sparkonten ausreichend diskutiert worden sind. Die vorgeschlagenen (ergänzenden) Maßnahmen zur Administration einer Konsumsteuer können nun zusammengefaßt werden:

1) Alle Einkünfte, die keine Erträge von Finanzanlagen darstellen, werden im Prinzip wie bisher erfaßt. Auf Lohneinkommen abzüglich der Rentenversicherungsbeiträge und abzüglich eines gewissen Anteils, der sich an der volkswirtschaftlichen Sparquote orientieren könnte, wird eine Lohnsteuer erhoben. Diese wird im Rahmen der jährlichen Konsumsteuerveranlagung, bei der die Lohnsteuerkarte vorzulegen ist, angerechnet. Die deklarierten Einkünfte (minus Einlagen) aus Gewerbebetrieb, Landwirtschaft und selbständiger Arbeit werden durch Betriebsprüfungen, die regelmäBiger als bisher stattfinden, festgestellt. Wie bei der Einkommensteuer gibt es hier vierteljährliche Vorauszahlungen auf die Konsumsteuer. Ob Mieten deklariert werden, wird mit Hilfe der Grundbucheintragungen und der existierenden Mietpreisspiegel überprüft. Bei all diesen Einkünften ergeben sich bei einer Hinterziehung strafrechtliche Konsequenzen.

2) Das Bankgeheimnis für Lebende in $\S 30$ a $\mathrm{AO}$ bleibt erhalten: Grundsätzlich erfolgt keine Überprüfung von Konten privater Haushalte. Sie erfolgt nur im Todesfall.

3) Bemessungsgrundlage der jährlichen Konsumsteuer mit direkt progressivem Tarif sind die unter 1) aufgeführten Nettoeinkünfte abzüglich der deklarierten Nettoeinzahlungen auf Sparkonten und Wertpapierdepots. Die maximal abziehbare deklarierte Ersparnis kann auf einen großzügig zu bemessenden Prozentsatz der unter 1) genannten Einkünfte beschränkt werden. Höhere Ersparnisse sind nachzuweisen. 
4) Für Zwecke der Besteuerung wird monatlich eine Durchschnittsrendite öffentlicher Anleihen berechnet.

5) Auf Antrag des Stpfl. nimmt das Finanzamt einen interperiodischen Progressionsausgleich vor. Das Verfahren ist oben in Abschnitt 5.3.3 dargestellt (siehe auch Fußnote 12). Es macht von den unter 4) angegebenen steuerlichen Zinssätzen Gebrauch.

6) Der Progressionsausgleich wird auf Initiative der Finanzbehörde durchgeführt, wenn das deklarierte Vermögen, d.h. die aufgezinsten deklarierten Ersparnisse eines längeren Zeitraums, im Vergleich zu den während dieses Zeitraums erzielten Einkünften groß ist und der Stpfl. nicht zustimmt, zur Deckung der latenten Steuerschuld Wertpapiere sicherheitshalber in einem Depot zu hinterlegen, von dem er sie nur mit Zustimmung des Finanzamtes abheben kann. Der Progressionsausgleich wird zusätzlich am Ende des Erwerbslebens eines Stpfl. durchgeführt.

\subsection{Abschließende Bemerkungen}

Es ist traditionelles Vorgehen in der Volkswirtschaftslehre, die Präferenzen der Haushalte als gegeben anzunehmen und nach institutionellen Arrangements zu suchen, in denen sie zur Geltung kommen können. An diese Methodik hat sich Kapitel 5 gehalten. Die bundesdeutschen Haushalte haben eine ausgeprägte Präferenz dafür, ihre privaten Sparkonten vor den Einblicken Dritter - unter anderem des Fiskus - geschützt zu wissen. Es fragt sich aber, ob ein Bankgeheimnis nicht ein Hindernis für die direkte Besteuerung darstellt.

Für die erfolgreiche Administration einer Konsumsteuer wäre die Prüfung von Sparkonten nicht notwendig. Insbesondere auf Kontrollmitteilungen über Finanzanlagen, die von Kaldor und anderen für unabdingbar gehalten wurden, kann verzichtet werden. Der Fiskus muß lediglich die Quellen der Ersparnisse eines Stpfl. feststellen können - eine Bedingung, die zum Beispiel für Haushalte, die überwiegend Lohneinkommen beziehen und von der Lohnsteuer erfaßt werden, erfüllt ist. Unter dieser Voraussetzung kann ein Stpfl. durch eine übertriebene Deklaration von Ersparnissen, die zunächst die jährliche Konsumsteuer mindern, auf die Dauer keinen Vorteil gewinnen, da die Finanzbehörden ihm auf der Basis der von ihm selbst angegebenen Ersparnisse in späteren Perioden einen höheren Konsum zurechnen werden.

Die bestehenden gesetzlichen Regelungen zur Steuersicherung in der Abgabenordnung stellen trotz des "Schutzes von Bankkunden" vor dem Fiskus in $\S 34$ a AO eine ausreichende Grundlage dar, um eine persönliche Ausgabensteuer zu 
erheben. Mieszkowski (1980, S. 180) ist zuzustimmen, wenn er feststellt, "the widely held view that the expenditure tax is too difficult to administer is simply false”. Die Einkommensteuer stößt dagegen auf Probleme, wenn der Fiskus die Zinserträge der privaten Haushalte nicht feststellen kann, weil diese einfach behaupten, sie hätten keine Ersparnisse. Ein Wechsel der Bemessungsgrundlage der direkten Besteuerung wäre jedoch nicht allein aus erhebungstechnischen Gründen zu befürworten, sondern auch aus Gründen der horizontalen und vertikalen Gerechtigkeit. Wenn man die intertemporale Budgetbeschränkung eines Haushalts als relevantes Kriterium eines Vergleichs von Steuerpflichtigen ansieht, schneidet die Einkommensteuer - mit und ohne Erfassung der Zinserträge - nicht gut gegen eine Konsumsteuer ab. Bei progressivem Jahrestarif bieten sich unter der Konsumsteuer einfache Verfahren an, um einen interperiodischen Progressionsausgleich herbeizuführen.

Die Konsumsteuer ist im Regierungsauftrag von Kaldor, Lodin und zwei Kommissionen untersucht worden (Kaldor, 1956, Lodin, 1982, Meade-Kommission, 1978, Bradford, 1984). Alle Gutachten schlossen zwar mit einem positiven Votum für die Konsumsteuer. In keinem Land ist sie aber ernsthaft umgesetzt worden (vgl. Thirlwall, 1987, Kap. 5, insbesondere S. 136 f., zu Kaldors Erfahrungen in Indien). Daß die Bundesrepublik hier zu einem Vorreiter werden könnte, klingt unwahrscheinlich. Mit der Einführung der Quellensteuer auf Zinserträge und ihrer Abschaffung nach halbjähriger Laufzeit hat sich die Finanzpolitik aber in eine Sackgasse gebracht, aus der ihr nur die Umstellung auf den Konsum als Bemessungsgrundlage der direkten Besteuerung hilft, wenn sie nicht zu intensiveren Kontrollen von Sparkonten oder/und höheren Strafen für die Steuerhinterziehung greifen will. 


\section{Kapitel 6}

\section{Steuerhinterziehung bei rückwirkenden Kontrollen und einer verschärften Prüfung Verdächtiger: Modelle mit wiederholter Veranlagung}

\subsection{Einleitung}

Die Arbeit hat sich mit der Ausnahme von Kapitel 5 auf Modelle eines Steuerpflichtigen beschränkt, der nur ein einziges Mal veranlagt wird. Ein für die Steuerpflichtigen fühlbares Merkmal aller Steuersysteme ist jedoch, daß sie jedes Jahr von neuem veranlagt werden. Zur Einkommensteuer zum Beispiel werden die Haushalte nicht ein- oder zweimal in ihrem Leben, sondern jedes Jahr veranlagt. Dieses Prinzip der Abschnittsbesteuerung ist gerade im Zusammenhang mit der Steuerhinterziehung nicht schwer zu verstehen. Es dient der Sicherung des Steuereingangs. Würde man nur ein einziges Mal zur Einkommensteuer veranlagt - am Ende des Berufslebens etwa -, bestünde der Anreiz, den Lebensabend in einem Niedrigsteuerland zu verbringen.

Das Prinzip der Abschnittsbesteuerung kann in Modellen der Steuerhinterziehung vernachlässigt werden, wenn man davon ausgehen kann, daß die Entscheidungen über das deklarierte Einkommen in aufeinander folgenden Jahren unabhängig voneinander getroffen werden können. Von dieser Annahme ist in den vorangehenden Kapiteln (bis auf Kapitel 5) stillschweigend ausgegangen worden. Nun kann es gerade aufgrund des Systems der Steuersicherung gute Gründe dafür geben, die Deklarationsentscheidungen in verschiedenen Jahren nicht unabhängig voneinander 
zu treffen: Die in der Zukunft zu erwartenden Kontrollen und Strafen können von dem heutigen Verhalten des Steuerpflichtigen beeinflußt werden, bzw. die heute durchgeführten Kontrollen und die heute gegebenenfalls zu tragenden Strafen sind von dem Verhalten des Steuerpflichtigen in der Vergangenheit abhängig. Die Literatur nennt zwei denkbare Verhaltensweisen der Finanzbehörden, die das System der Steuersicherung von dem Verhalten der Steuerpflichtigen abhängig machen:

- Rückwirkende Kontrollen und Strafen (Allingham/Sandmo, 1972) Entdeckt die Behörde, daß der Steuerpflichtige in dem laufenden (bzw. gerade zu Ende gegangenen) Jahr Steuern hinterzogen hat, werden auch die Steuererklärungen in den zurückliegenden Jahren eine nach der anderen überprüft - und zwar solange, bis sie auf ein Jahr stößt, in dem entweder schon einmal eine Prüfung stattgefunden hat oder in dem der Steuerpflichtige sein tatsächliches Einkommen deklariert hat. Eine nachträglich entdeckte Steuerverkürzung führt zu rückwirkenden Strafen.

- Verschärfte Kontrollen Verdächtiger (Landsberger/Meilijson, 1982, Greenberg, 1984, Harrington, 1988)

Wer heute der Steuerhinterziehung überführt wird, muß in den nächsten Jahren damit rechnen, daß er mit einer höheren Wahrscheinlichkeit von der Steuerfahndung geprüft wird. Erst wenn einmal festgestellt wird, daß ein Verdächtiger steuerehrlich geworden ist, sinkt die Entdeckungswahrscheinlichkeit eventuell wieder.

Beide "Daumenregeln" sind für Finanzbehörden plausibel, die mit denen ihnen zur Verfügung stehenden Mitteln ein möglichst hohes Aufkommen erzielen sollen.

Die Literatur zu Modellen mit wiederholter Veranlagung hat die zwei angeführten Politiken der Finanzbehörden mit unterschiedlicher Aufmerksamkeit bedacht. $\mathrm{Zu}$ der Einteilung der Steuerpflichtigen in Verdächtige und weniger Verdächtige gibt es drei Veröffentlichungen. Rückwirkende Kontrollen sind meines Wissens dagegen im Anschluß an Allingham/Sandmo (1972, S. 332 - 337) nur von Rickard u.a. (1982) aufgegriffen worden. Da Rickard u.a. (1982, S. 380) jedoch von der nicht begründbaren Annahme ausgehen, daß der Steuerpflichtige in jedem Jahr den gleichen Anteil seines Einkommens hinterzieht, nehmen sie ihrem Aufsatz einen GroBteil des Interesses, wie unten deutlich werden wird. Allingham/Sandmo lassen dagegen zu, daß der Steuerpflichtige seine Deklarationsentscheidung von Periode zu Periode ändert, und kommen zu dem Ergebnis, daß es für ihn auch von Vorteil ist, dies zu tun. Ihre Analyse ist ohne Frage interessant - aber sehr kurz und nicht leicht nachzuvollziehen, da sie aus einer Mischung von Modell und Intuition besteht.

Die Ziele dieses Kapitels sind 
- die systematische Untersuchung des Verhaltens eines Steuerpflichtigen, der mit rückwirkenden Kontrollen rechnen muß,

- und die Darstellung der Modelle, in denen der Hinterziehung besonders verdächtige Steuerpflichtige verschärft kontrolliert werden.

In Abschnitt 6.2 wird die Methode, mit der Harrington (1988) von dem Verhalten des Steuerpflichtigen abhängige Entdeckungswahrscheinlichkeiten berücksichtigt, verwendet, um von dem Verhalten des Steuerpflichtigen abhängige Strafen zu modellieren. Dabei wird mit der Verjährung der Steuerhinterziehung ein zusätzlicher Grund für den Abbruch der rückwirkenden Kontrollen und Strafen eingeführt: Nach Ablauf der Verjährungsfrist kann die Steuerhinterziehung nicht mehr rückwirkend bestraft werden. Da die Methode - der Markov-Entscheidungsprozeß -, mit der man Modelle mit wiederholter Veranlagung formulieren und auswerten kann, in Abschnitt 6.2 ausführlich dargestellt wird, kann sich Abschnitt 6.3 darauf beschränken, die Modelle mit Klassen verdächtiger und weniger verdächtiger Steuerpflichtiger relativ knapp darzustellen.

Modelle mit wiederholter Veranlagung sind aus zwei Gründen interessant:

1) Der Leser sieht es als gut möglich an, daß Strafen und Entdeckungswahrscheinlichkeiten von dem Verhalten der Steuerpflichtigen abhängig gemacht werden, und möchte wissen, wie ein rationaler Steuerzahler sich am besten verhält.

Die zentrale Frage ist hier, wie die zeitliche Entwicklung des hinterzogenen Einkommens aussieht. Gibt es Jahre, in denen der Steuerpflichtige sein tatsächliches Einkommen deklariert? Daran schließt sich die komparativ-dynamische Frage an, wie die Sequenz der Deklarationsentscheidungen von bestimmten Parametern abhängt: Wie verhält sich der Steuerpflichtige zum Beispiel, wenn die Verjährungsfrist für die Steuerhinterziehung verlängert wird?

2) Aus der Sicht einer Finanzbehörde, die mit knappen Mitteln ein möglichst hohes Aufkommen erzielen will, stellt sich eine zweite Frage: Lohnt es sich, die Kontrollen und Strafen von dem Verhalten des Steuerpflichtigen abhängig zu machen, statt jede Steuererklärung mit der gleichen Wahrscheinlichkeit zu überprüfen und immer nur die Hinterziehung des laufenden Einkommens zu bestrafen?

Die Finanzbehörden haben von dem Gesetzgeber den Auftrag erhalten, "die Steuern nach Maßgabe der Gesetze gleichmäßig festzusetzen und zu erheben. Insbesondere haben sie sicherzustellen, daß Steuern nicht verkürzt ... werden” ( $\S 85$ AO). Die Mittel, die die Finanzbehörden zur Erfüllung dieses Auftrags erhalten, 
sind jedoch begrenzt. Auf jeden Fall scheinen sie nicht groß genug zu sein, um die Steuerpflichtigen in einem statischen System der Steuersicherung zu veranlassen, ihr Einkommen wahrheitsgemäß anzugeben. Die Bedingung $(1-p)-p \alpha \leqq 0$ verlangt selbst bei einer Strafe von $3 \mathrm{DM}$ für jede DM hinterzogener Steuern $(\alpha=3)$ mindestens eine Entdeckungswahrscheinlichkeit $p=0,25$. Jedes Jahr ein Viertel der steuerpflichtigen Bevölkerung aufzusuchen, wird das Budget der Finanzverwaltung aber nicht zulassen. Der Ausweg, den Strafsatz $\alpha$ zu erhöhen, bis die angegebene Bedingung erfüllt ist, ist versperrt: Sehr hohe Strafen für ein "Kavaliersdelikt" dürften auf massiven Widerstand der Bürger stoßen. Es stellt sich daher die Frage, ob ein dynamisches System der Steuersicherung, in dem Strafen und Entdeckungswahrscheinlichkeiten von Gestzesverstößen in der Vergangenheit abhängig sind, unter den Beschränkungen eines knappen Budgets der Finanzbehörden und einer niedrigen Grenze für den Strafsatz $\alpha$ nicht eher als das statische System geeignet ist, die Steuern nach Maßgabe der Gesetze zu erheben.

Die Beantwortung dieser Fragen ist interessant genug, um einen Preis zu zahlen: Die vorhandenen Modelle mit wiederholter Veranlagung gehen überwiegend von einem risikoneutralen Steuerpflichtigen aus und nehmen ein exogenes, möglicherweise aber zufälliges Einkommen an. Zwischen den Modellen mit wiederholter Veranlagung und einmaliger Veranlagung besteht daher ein gewisser Bruch. Man muß jedoch folgendes sehen. Die Analyse der einmaligen Veranlagung wäre ohne die Annahme der Risikoversion schnell uninteressant. Modelle mit wiederholter Veranlagung sind auch mit risikoneutralen Steuerpflichtigen interessant - was sicher hinreichend ist zur Rechtfertigung dieser Annahme.

\subsection{Rückwirkende Kontrollen}

Eine rückwirkende Kontrolle endet bei Allingham/Sandmo (1972) aus zwei Gründen: Die Steuerfahndung stößt auf eine Periode, in der der Stpfl. ehrlich war oder in der er schon einmal geprüft worden ist. Im letzten Fall ist eine nochmalige Prüfung überflüssig. Im ersten Fall unterstellt die Behörde u.U., daß der Stpfl. in noch weiter zurückliegenden Jahren ebenfalls ehrlich war. Es soll hier ein weiterer Grund für den Abbruch der rückwirkenden Kontrolle angenommen werden: Durch eine Verjährung erlöschen alle Ansprüche des Staates. Eine Steuerhinterziehung, die länger als die Verjährungsfrist zurückliegt, führt weder zu einer Nachzahlung noch zu Geldstrafen. Die Steuerfahndung wird nach Ablauf der Verjährungsfrist aufhören, die Vergangenheit des Stpfl. aufzurollen, da der zusätzlichen Arbeit dann kein "Mehrergebnis" gegenübersteht.

Alle drei Gründe für den Abbruch der rückwirkenden Kontrollen lassen sich auf einen Nenner bringen: Die Prüfungen enden, sobald die Behörde auf eine 
Periode stößt, in der die Kontrolle erstmals kein zusätzliches Aufkommen einbringt (vgl. Allingham/Sandmo, 1972, S. 334). Diese "Daumenregel" ist plausibel für eine Steuerfahndung, die mit knappem Personal ein möglichst hohes Aufkommen erzielen will.

Zu der Verjährung der Steuerhinterziehung nach deutschem Recht sollen einige Hinweise festgehalten werden. Zu unterscheiden sind die Verjährung des Steueranspruchs und die Strafverfolgungsverjährung. Die Abgabenordnung nennt die Verjährung des Steueranspruchs Festsetzungsverjährung. Sie beginnt bei der Einkommensteuer mit Ablauf des Jahres, in dem die Steuererklärung eingereicht worden ist ( $(170 \mathrm{Abs} .2 \mathrm{AO}$ ). Sie beträgt zehn Jahre, soweit eine Steuer hinterzogen worden ist ( $\$ 169$ Abs. 2 AO). Der Ablauf der Festsetzungsfrist wird jedoch aus verschiedenen Gründen gehemmt. Sie endet insbesondere nicht, bevor die Verfolgung der Steuerstraftat verjährt ist ( $\$ 171$ Abs. 7 AO). Solange eine Bestrafung möglich ist, kann die verkürzte Steuer festgesetzt werden. Die Strafverfolgung der Steuerhinterziehung verjährt in fünf Jahren (vgl. Franz/Gast/Samson, 1985, S. 485). Durch die Annahme eines Fortsetzungszusammenhangs kann sie aber weit über diese Frist ausgedehnt werden. Die fortgesetzte Steuerhinterziehung über mehrere Jahre wird als eine einzige Straftat betrachtet, wenn das Gericht den Eindruck gewinnt, daß der Stpfl. "den Gesamtvorsatz hatte, Jahr für Jahr Steuern zu hinterziehen" (Streck, 1986, S. 233). Da die Verjährung nach $\S 78$ a Satz 1 StGB mit der Beendigung der Tat einsetzt, beginnt die Verjährung jedes Teilaktes einer "Serie" von Steuerhinterziehungen dann erst mit der letzten unrichtigen Veranlagung. Daß ein Stpfl. einen Gesamthinterziehungsvorsatz hatte, kann jedoch oft nicht nachgewiesen werden (vgl. Streck, 1986, S. 233). Zudem wird der Zweck der Verjährung, Rechtsfrieden und -sicherheit herzustellen, durch die Annahme eines Fortsetzungszusammenhangs unterlaufen (siehe ebenda, S. 233, für Hinweise zur Kritik des Fortsetzungszusammenhangs).

Im folgenden wird davon ausgegangen, daß die Stpfl. im Interesse des Rechtsfriedens jeweils mit einer ganz bestimmmten Festsetzungs- und Strafverfolgungsverjährung rechnen können. Zusätzlich seien beide Fristen gleich lang.

Teil 2 dieses Kapitels gliedert sich wie folgt. In den Abschnitten 6.2.1 und 6.2.2 wird ein Modell mit einer Verjährungsfrist von einem Jahr und einem festen Einkommen dargestellt. Die Abschnitte 6.2.3 und 6.3.4 verallgemeinern das Modell für eine beliebige Verjährungsfrist und ein stochastisches Einkommen. In Abschnitt 6.2.5 geht es um die Frage, ob sich die Einführung rückwirkender Kontrollen für die Finanzbehörden lohnt. 


\subsubsection{Das Modell mit einer Verjährungsfrist von einem Jahr}

Es wird zunächst angenommen, daß

1. die Steuerhinterziehung nach einem Jahr verjährt ist.

Der Stpfl. kann also maximal für die Hinterziehung im laufenden (bzw. gerade zu Ende gegangenen) Jahr und im Vorjahr belangt werden. Die übrigen Annahmen des Modells und kurze Bemerkungen zu ihren Implikationen enthält die folgende Liste.

2. Der Stpfl. lebt unendlich viele Perioden und wird in jeder Periode veranlagt. Rückwirkende Strafen werden erst dann interessant, wenn der Stpfl. mindestens zweimal hintereinander veranlagt wird. Gegenüber endlich vielen Veranlagungsperioden haben unendliche viele Perioden - wie oft in der ökonomischen Theorie analytische Vorteile, die auch in dieser Arbeit genutzt werden sollen. In der Praxis wird ein Stpfl. zwar nicht unendlich oft, aber jahrzehntelang jedes Jahr veranlagt, so daß man das im folgenden dargestellte Modell als Näherung betrachten kann.

3. Der Stpfl. ist risikoneutral.

Diese Annahme wird die Folge haben, daß der Stpfl. bei seinen Entscheidungen über sein deklariertes Einkommen jeweils Ecklösungen wählt.

4. Der Stpfl. diskontiert zukünftige Einkommen nach Steuer und Strafen mit dem Faktor $\eta, 0<\eta<1$.

Der Diskontfaktor $\eta$ ist gleich $1 /(1+\phi)$, wobei $\phi$ der Zinssatz ist, mit dem der Stpfl. zukünftige Einkommen diskontiert.

5. In jeder Periode verfügt der Stpfl. über ein gegebenes Einkommen $Y$, das er dem Finanzamt angeben muB, da es einer Einkommensteuer mit dem Tarif $T(Y)$ unterliegt. $Y$ sei so groß, daß die maximal verkürzbare Steuer $1 \mathrm{DM}$ beträgt. Für die in Jahr $t$ hinterzogene Steuer $S_{t}$ gilt damit die Beschränkung $0 \leqq S_{t} \leqq 1$.

6. Die Angaben des Stpfl. werden in jeder Periode mit der Wahrscheinlichkeit $p$ kontrolliert $(0<p<1)$. Die Prüfung deckt zuerst eine eventuelle Hinterziehung in der laufenden Periode auf. Hat der Stpfl. nicht sein tatsächliches Einkommen deklariert, wird geprüft - soweit nicht schon in der Vorperiode geschehen -, ob das in dem Vorjahr deklarierte Einkommen den Tatsachen entsprochen hat.

\footnotetext{
${ }^{1}$ In Kapitel 6 bezeichnet $t$ stets die Periode.
} 
7. Hinterzogene Steuern sind nachzuzahlen. Die zusätzliche Geldstrafe ist ein konstantes Vielfaches $\alpha$ der hinterzogenen Steuern. Die Nachzahlung und die Strafe, die sich auf das Vorjahr beziehen, sind zu verzinsen.

8. Es gilt $(1-p)-p \alpha=q-p \alpha>0$.

9. Der Stpfl. maximiert durch die Wahl von $S_{t}$ für jede Periode $t$ seines Lebens $(t=0,1,2, \ldots)$ den Barwert seines erwarteten Einkommens nach Steuer und Strafe.

Der Stpfl. kann wohlgemerkt in jeder Periode einen anderen Betrag $S_{t}$ wählen. Er muß nicht stets den gleichen Anteil seines Einkommens hinterziehen.

Annahme 9 läßt sich auch anders ausdrücken. Da das Einkommen gegeben ist, kann man sagen, daß statt des Barwertes der erwarteten Nettoeinkommen der Barwert aller erwarteten Erträge der Steuerhinterziehung maximiert wird.

Wie lauten die Budgetbeschränkungen des Stpfl. in den zwei Zuständen "entdeckt" und "nicht entdeckt"? Wird er nicht geprüft, ist sein Nettoeinkommen in einer bestimmten Periode $t$ gleich

$$
G_{t}=Y-T(Y)+S_{t}
$$

Wird der Stpfl. kontrolliert, sind zwei Fälle zu unterscheiden. Wenn er auch in der Vorperiode geprüft worden ist und für eine eventuelle Hinterziehung $S_{t-1}$ schon "bezahlt" hat, ist sein Nettoeinkommen

$$
\tilde{P}_{t}=Y-T(Y)-\alpha S_{t}
$$

Wenn er in der Vorperiode nicht geprüft worden ist, beträgt sein Nettoeinkommen

$$
\hat{P}_{t}= \begin{cases}Y-T(Y), & \text { wenn } S_{t}=0 \\ Y-T(Y)-\alpha S_{t}-(1+\alpha) S_{t-1} \eta^{-1}, & \text { wenn } S_{t}>0\end{cases}
$$

Wenn der Stpfl. ehrlich ist - die Prüfung der Steuererklärung für das laufende Jahr also kein Mehrergebnis einbringt -, wird seine Vergangenheit nicht untersucht. Mit $S_{t}>0$ wird auch die Steuererklärung des Jahres $t-1$ überprüft und die Hinterziehung $S_{t-1}$ entdeckt, so daß eine zusätzliche Nachzahlung und Strafe anfallen. Die Budgetbeschränkung für den Fall einer Kontrolle hängt damit von der jüngeren Vergangenheit ab - wurde in $t-1$ geprüft? - und weist eine Sprungstelle auf, falls in Periode $t-1$ nicht kontrolliert worden ist.

$\mathrm{Zu}$ dem Einkommen $\hat{P}_{t}$ fehlt noch eine Bemerkung. Die Nachzahlung der in Jahr $t-1$ verkürzten Steuer wird verzinst, da $\S 235$ AO bestimmt: Hinterzogene Steuern sind zu verzinsen. Die Verzinsung der Strafe soll damit gerechtfertigt 
werden, daß der Barwert der Strafe für die Hinterziehung in einer bestimmten Periode unabhängig davon sein soll, zu welchem Zeitpunkt das Vergehen aufgedeckt wird. Der Einfachheit halber ist unterstellt, daß Strafe und Nachzahlung mit dem Zinssatz verzinst werden, mit dem der Stpfl. sein Einkommen diskontiert.

Die Annahmen 1 - 9 werden nun in ein Modell - speziell einen MarkovEntscheidungsproze $B$ - übersetzt. Da dieses Instrumentarium in den vorangehenden Kapiteln keine Verwendung gefunden hat, wird die Übersetzung unseres ökonomischen Problems Schritt für Schritt vor sich gehen.

$\mathrm{Da}$ mit einer rückwirkenden Kontrolle die jüngere Vergangenheit des Stpfl. eine Rolle spielt, denkt er daran, daß er sich in Periode $t$ in genau einem von drei Zuständen befindet, bevor er seine Steuererklärung für diese Periode abgibt. Die Bezeichnung, die Beschreibung und einen Namen dieser drei Zustände enthält folgende Liste:

s: Der Stpfl. war in Jahr $t-1$ steuerehrlich ("Start").

$m$ : Der Stpfl. hat in $t-1$ hinterzogen und wurde entdeckt ("Mißerfolg").

$e$ : Der Stpfl. hat in $t-1$ hinterzogen und wurde nicht entdeckt. Auch in $t-2, t-3$, etc. hat er möglicherweise hinterzogen und wurde nicht entdeckt. Ist er in Zustand $e$, hat er in der jüngsten Vergangenheit also mindestens einmal hintereinander erfolgreich Steuern verkürzt, nachdem er vor dieser Erfolgsserie entweder ehrlich war oder entdeckt worden ist ("Erfolg").

Sei $Z=\{s, m, e\}$ die Menge aller Zustände. Sei $\left(S_{y t}\right)$ ein Vektor, der für jeden Zustand $y \in Z$ angibt, ob in Periode $t$ alle oder keine Steuern verkürzt werden. Es kann hier angenommen werden, daß die hinterzogene Steuer in Zustand $y$ in Jahr $t, S_{y t}$, entweder eins oder null ist. Unten wird gezeigt, daß diese Annahme eine Konsequenz der Risikoneutralität des Stpfl. ist. Die Matrix der Übergangswahrscheinlichkeiten $\left(p_{y z}\left[\left(S_{y t}\right)\right]\right), y, z \in Z$, gibt an, mit welcher Wahrscheinlichkeit der Stpfl. von einem Zustand $y$ in Periode $t$ in einen Zustand $z$ in Periode $t+1$ übergeht. Die Übergangswahrscheinlichkeiten sind abhängig von den Entscheidungen $\left(S_{y t}\right)$. Die Matrix der Erträge $\left(r_{y z}\right), y, z \in Z$, gibt an, was der Stpfl. relativ zu seinem sicheren Nettoeinkommen $Y-T(Y)$ gewinnt oder verliert, wenn er von Zustand $y$ in Zustand $z$ übergeht.

Nehmen wir zunächst einmal an, daB in jeder Periode 1 DM Steuern hinterzogen wird, egal in welchem Zustand der Stpfl. jeweils ist: $\left(S_{v t}\right)=(111), t=0,1,2, \ldots$. Die zwei Matrizen sind dann für alle $t$ :

$$
\left(p_{y z}\left[\left(S_{y t}\right)=(111)\right]\right)=\underset{s}{s}\left(\begin{array}{lll}
s & m & e \\
e & p & q \\
0 & p & q \\
0 & p & q
\end{array}\right)
$$


Abbildung 6.1: Die möglichen Wege eines notorischen Hinterziehers in den ersten drei Perioden

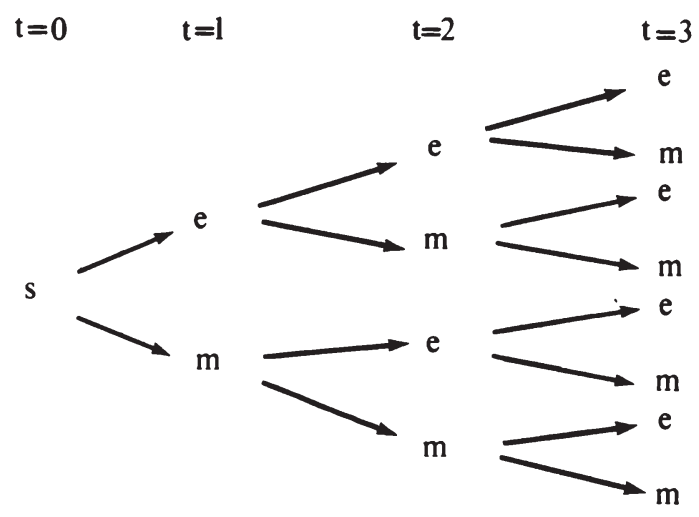

und

$$
\left(r_{y z}\right)=\stackrel{s}{m} \underset{e}{s}\left(\begin{array}{ccc}
0 & m & e \\
0 & -\alpha & 1 \\
0 & \left(-\alpha-(1+\alpha) \eta^{-1}\right) & 1
\end{array}\right)
$$

Die Zustände sind der Übersicht halber an den Seiten der Matrizen festgehalten. Startet der Stpfl. in Periode $t=0$ in Zustand $s$, gelangt er mit der Wahrscheinlichkeit $q$ nach $e$ und mit der Wahrscheinlichkeit $p$ nach $m$. Bei diesen Übergängen gewinnt er $1 \mathrm{DM}$ oder er verliert $\alpha$. Sein erwarteter Ertrag ist $q-p \alpha$. Hat er Erfolg gehabt und befindet er sich nun in $e$, geht er mit der Wahrscheinlichkeit $p$ in $m$ über und verliert dabei $\alpha+(1+\alpha) \eta^{-1}$. Mit der Wahrscheinlichkeit $q$ bleibt der Stpfl. in Zustand $e$ : Wenn er dreimal (und öfter) hintereinander hinterzieht, ist die Strafe nicht größer, als wenn er zweimal hintereinander kein Einkommen deklariert. Aufgrund der Verjährung kann der Stpfl. mit der Wahrscheinlichkeit $q$ in Zustand $e$ verbleiben.

Wenn $S_{y t}=1$ entdeckt wird, ist der Stpfl. jeweils wieder in derselben Situation wie "am Start" in $s$. Von $m$ aus kann er nur nach $e$ und $m$ übergehen. Diese Übergänge sind jeweils mit den gleichen Erträgen verbunden, wie die Übergänge von Zustand $s$ nach $m$ und $e$. Abbildung 6.1 zeigt alle möglichen Wege eines Stpfl., der sein Einkommen nie wahrheitsgemäß deklariert, in den ersten drei Perioden. Dabei ist angenommen, da $B$ er in Zustand $s$ startet. Einem Pfeil, der nach oben 
Abbildung 6.2: Die möglichen Wege eines Hinterziehers, der nur in Zustand $e$ ehrlich wird, in den ersten drei Perioden

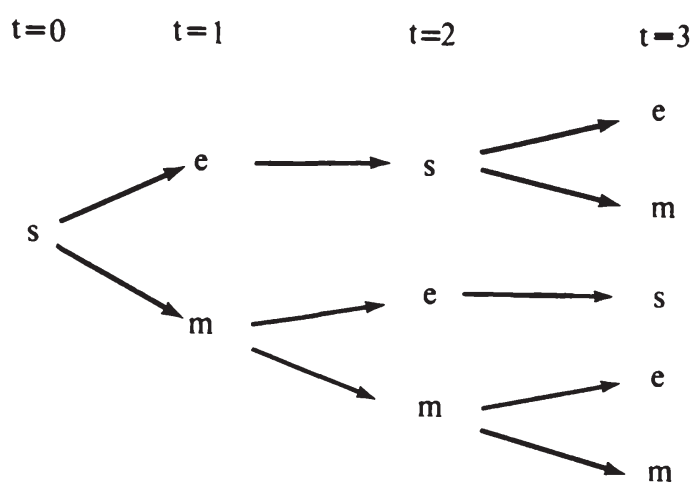

(unten) zeigt, folgt der Stpfl. jeweils mit der Wahrscheinlichkeit $q(p)$.

Wenn der Stpfl. nie sein tatsächliches Einkommen deklariert, kann er nicht in Zustand $s$ zurückkehren. Alle Elemente der ersten Spalte der angegebenen Matrix der Übergangswahrscheinlichkeiten sind daher null. Wenn er jedoch sein Einkommen wahrheitsgemäß deklariert, gelangt er von jedem Zustand $y \in Z$ mit der Wahrscheinlichkeit eins nach $s$ : Wenn er einmal ehrlich ist, hat er seine Vergangenheit hinter sich gelassen. Dies gilt auch für den Fall, daß die Finanzbehörde den Stpfl. gar nicht kontrolliert. Auch eine spätere rückwirkende Prüfung wird ja beendet werden, sobald eine Periode gefunden wird, in der nicht hinterzogen worden ist. In der Matrix der Übergangswahrscheinlichkeiten, die oben angegeben ist, können also in jeder einzelnen Zeile $q$ und $p$ durch null ersetzt und es kann stattdessen an den Anfang der Zeile eine Eins gestellt werden. Die Zeilensummen der stochastischen Matrix blieben bei dieser Operation gleich eins: In irgendeinen der möglichen Zustände muß der Stpfl. ja übergehen, solange der stochastische Prozeß weitergeht. Wenn der Stpfl. z.B. ehrlich wird, sobald er gerade einmal erfolgreich war, im übrigen aber stets kein Einkommen deklariert, erhält man die Matrix

$$
\left(p_{y z}\left[\left(S_{y t}\right)=(110)\right]\right)=\underset{s}{s}\left(\begin{array}{lll}
s & m & e \\
e & p & q \\
0 & p & q \\
1 & 0 & 0
\end{array}\right)
$$

Die möglichen Wege eines Stpfl., der in Zustand $e$ stets ehrlich ist und in den 
Zuständen $s$ und $m$ immer hinterzieht, zeigt Abbildung 6.2 für die ersten drei Perioden. Einem waagerechten Pfeil folgt der Stpfl. mit der Wahrscheinlichkeit eins. Die Wahrscheinlichkeit, einen Schritt nach oben (unten) zu tun, ist wieder gleich $q(p)$.

Legt der Stpfl. für jeden Zeitpunkt $t=0,1,2, \ldots$ und jeden Zustand $y \in Z$ fest, ob $S_{y t}$ gleich null oder eins sein soll, und befindet er sich zu $t=0$ in $s$, wird sein Werdegang in dem Zustandsraum $Z$ durch eine Markov-Kette mit den Übergangswahrscheinlichkeiten $\left(p_{y z}\left[\left(S_{y t}\right)\right]\right), t=0,1,2, \ldots$, und der Anfangsverteilung "mit der Wahrscheinlichkeit eins befindet sich der Stpfl. zu $t=0$ in $s$ " vollständig beschrieben. Die Folge der Zufallsvariablen "Zustand, in dem sich der Stpfl. in Periode $t$ befindet", $t=0,1,2, \ldots$, genügt der Markov-Eigenschaft: Die Wahrscheinlichkeit, daß der Stpfl. in der nächsten Periode in Zustand $z$ gelangt, hängt (bei gegebenem $S_{y t}$ ) nur von dem Zustand $y \mathrm{ab}$, in dem er sich gerade befindet. Die Übergangswahrscheinlichkeiten $p_{y z}\left(S_{y t}\right)$ sind unabhängig von der übrigen Vorgeschichte des stochastischen Prozesses. Anschaulich ausgedrückt heißt das: Die zukünftige Entwicklung, die der Stpfl. durchläuft, ist nur von dem Zustand abhängig, in dem er sich gegenwärtig befindet, unabhängig aber von seiner Vergangenheit. Diese Eigenschaft ist wichtig, weil man ohne sie nur wenig über einen stochastischen Prozess aussagen kann.

In einem Modell der Steuerhinterziehung mit rückwirkenden Kontrollen ist es zunächst überraschend, die Markov-Eigenschaft anzutreffen. Wie weit die Überprüfung von Veranlagungen der Vergangenheit geht, hängt doch gerade von der Vorgeschichte des Stpfl. ab. Aus der Theorie stochastischer Prozesse ist jedoch bekannt, "that essentially every stochastic process can be viewed as a Markov process" (Heyman/Sobel, 1984, S. 105), wenn man den Zustandsraum nur so weit definiert (bzw. definieren kann), daß die relevante Vergangenheit immer in dem gegenwärtigen Zustand zusammengefaßt ist.

\subsubsection{Das Markov-Entscheidungsproblem des Steuerpflichtigen}

In diesem Abschnitt wird die Politik ermittelt, die den Kapitalwert des erwarteten Ertrages der Steuerhinterziehung maximiert. Eine Politik gibt für jeden Zustand und jeden Zeitpunkt den Wert von $S_{y t} \in\{0,1\}$ an. Es gibt also recht viele Politiken, zwischen denen der Stpfl. wählen muß. Diese Wahl wird durch folgendes Theorem und eine einfache Überlegung erleichtert.

Wenn der Diskontfaktor $\eta$ kleiner eins ist, die Matrix der Erträge $\left(r_{y z}\right)$ nicht von $t$ abhängt und ihre Elemente endlich sind, gilt (Kohlas, 1978, S. $133-141$ ):

(i) Der Kapitalwert des erwarteten Ertrags der Steuerhinterziehung ist bei jeder 
(zulässigen) Politik endlich.

(ii) Die optimale Politik ist stationär: $S_{y t}^{*}=S_{y}^{*}$ für alle $t$. Die Entscheidung $S_{y t}^{*}$ hängt nur von dem Zustand $y$, nicht aber von der Periode $t$ ab.

(iii) Die optimale Politik ist unabhängig von dem Anfangszustand des Stpfl.

Punkt (ii) erklärt sich damit, daß sich in jedem Zustand immer wieder das gleiche Entscheidungsproblem stellt (Kohlas, 1978, S. 136). Der Grund für (iii) ist, daß nur die Höhe des Kapitalwertes einer Politik von dem Zustand abhängt, in dem sich der Stpfl. gerade befindet, nicht aber die Reihenfolge der Kapitalwerte verschiedener Politiken.

Die Suche nach der Politik, die den höchsten Kapitalwert hat, kann sich auf die Festlegung des dreistelligen Vektors $\left(S_{y}\right)$ beschränken, der für jeden Zustand $y \in Z$ eine Null oder eine Eins enthält. Die Menge der in Frage kommenden Politiken läßt wegen der Annahme $q-p \alpha>0$ weiter reduzieren:

a) In $s$ wird der Stpfl. sein Einkommen hinterziehen. Die Steuerehrlichkeit zahlt sich in diesem Zustand nicht aus.

b) In $m$ stellt sich das gleiche Entscheidungsproblem wie in $s$, da eine rückwirkende Strafe nicht zu befürchten ist. Für die Vergangenheit ist bereits bezahlt worden. In $m$ wird der Stpfl. daher ebenfalls hinterziehen.

Es bleiben zwei Politiken übrig: $\left(S_{y}\right)^{n}=(111)$ und $\left(S_{y}\right)^{1}=(110)$. Die erste Politik verfolgt ein notorischer Steuerhinterzieher. Er ist nie steuerehrlich. Einen Stpfl., der die zweite Politik verfolgt, bezeichne ich als periodischen Hinterzieher. Sobald er genau einmal erfolgreich war, sichert er diesen Erfolg, indem er einmal ehrlich ist. Die Übergangswahrscheinlichkeiten der zwei Politiken $\left(S_{y}\right)^{n}$ und $\left(S_{y}\right)^{1}$ wollen wir noch einmal festhalten:

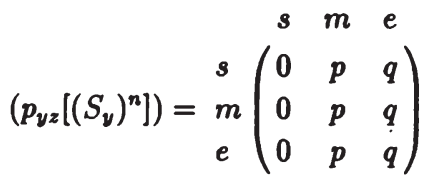

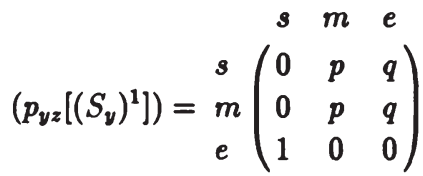

Es werden nun die Erträge der zwei Politiken berechnet. Seien $N(y)$ und $E(y)$ die Kapitalwerte der erwarteten Erträge der Steuerhinterziehung aller Perioden, 
wenn die Politik $\left(S_{y}\right)^{n}$ bzw. $\left(S_{y}\right)^{1}$ verfolgt wird und der Stpfl. zu Anfang in Zustand $y$ ist. Da der Stpfl. einen unendlichen Planungshorizont hat und er unter stationären Rahmenbedingungen eine stationäre Politik wählt, sind diese Kapitalwerte unabhängig von der Zeit. Diese Eigenschaft erlaubt es, $N(y)$ und $E(y)$ mit Hilfe von zwei einfachen Gleichungssystemen zu berechnen.

Es wird zuerst der notorische Hinterzieher behandelt. Alle Gleichungen haben folgende Struktur: Der Kapitalwert der Steuerhinterziehung in einem Zustand y ist gleich dem erwarteten Ertrag der Hinterziehung des laufenden Einkommens plus dem mit $\eta$ multiplizierten erwarteten Kapitalwert der Steuerhinterziehung in der nächsten Periode ${ }^{2}$. Die zwei ersten Gleichungen sind

$$
\begin{gathered}
N(s)=q-p \alpha+q \eta N(e)+p \eta N(m) \\
N(e)=q-p \alpha-p(1+\alpha) \eta^{-1}+q \eta N(e)+p \eta N(m)
\end{gathered}
$$

Betrachten wir die erste dieser Gleichungen. Der Kapitalwert der Steuerhinterziehung in Zustand $s$ hat zwei Komponenten:

- Mit der Wahrscheinlichkeit $q$ erhält der Stpfl. den Betrag 1 und ist in der nächsten Periode in Zustand $e$, so $\mathrm{daB}$ er den Kapitalwert $N(e)$ auf die laufende Periode diskontieren muß.

- Mit der Wahrscheinlichkeit $p$ verliert der Stpfl. $\alpha$ und ist in der nächsten Periode in $m$, so daß er alle zukünftigen Erträge mit $\eta N(m)$ zusammenfassen kann.

Die zweite Gleichung ist entsprechend zu interpretieren. Eine weitere Beziehung fehlt noch:

$$
N(m)=N(s)
$$

In $m$ besteht dieselbe Ausgangssituation wie in $s$. Also müssen auch die jeweiligen Kapitalwerte übereinstimmen.

Es genügt, den Ertrag der Steuerhinterziehung "am Start" in $s$ zu berechnen. Nummer (iii) des oben angeführten Theorems impliziert, daß der Vergleich von $N(y)$ und $E(y)$ für alle $y$ zu demselben Ergebnis führt. Setzt man (6.3) in (6.1) und (6.2) ein und löst nach $N(s)$ auf, ergibt sich

$$
N(s)=\frac{q-p \alpha-q p(1+\alpha)}{1-\eta}
$$

\footnotetext{
${ }^{2}$ Das folgende Beispiel hat ebenfalls diese Struktur: Sei $K$ der Kurs eines Konsols kurz vor dem jährlichen Zinstermin, $z$ die Zinsen, $\phi$ der Marktzins und $\eta=1 /(1+\phi)$. Der Kurs in dieser Periode ist gleich den Zinsen plus dem diskontierten Kurs der nächsten Periode: $K=z+\eta K$. Die Auflösung nach $K$ ergibt: $K=z /(1-\eta)$.
} 
Dieser Kapitalwert ist nicht schwer zu interpretieren. Wenn $S_{y t}$ in jedem Zeitpunkt und Zustand gleich eins ist, gibt der Zähler von (6.4) den erwarteten Ertrag an, der der Hinterziehung in jeweils einer Periode zuzurechnen ist: Mit der Wahrscheinlichkeit $p$ verliert der Stpfl. sofort $\alpha$ DM. Mit der Wahrscheinlichkeit $q$ gewinnt er zunächst 1 DM. Mit der Wahrscheinlichkeit $q p$ geht dieser Gewinn jedoch in der nächsten Periode verloren, wobei zusätzlich noch die rückwirkende Strafe zu zahlen ist. Den Nenner von (6.4) erhellt die letzte Fußnote.

Lohnt es sich bei einer Politik, bei der in jeder Periode ein positiver Betrag hinterzogen wird, dem Finanzamt in irgendeiner Periode mehr als null DM zu deklarieren? Würde der Stpfl. z.B. in der ersten Periode nicht $1 \mathrm{DM}$, sondern nur $1 / 2 \mathrm{DM}$ hinterziehen, würde $N(s)$ um $(q-p \alpha-q p(1+\alpha)) / 2$ sinken. Ist der Zähler von (6.4) positiv, wird der notorische Hinterzieher stets sein gesamtes Einkommen hinterziehen.

Der Ertrag des Steuerhinterziehers, der nach einem Erfolg eine kurze Pause einlegt, ergibt sich aus folgendem Gleichungssystem:

$$
\begin{aligned}
E(s) & =q-p \alpha+q \eta E(e)+p \eta E(m) \\
E(e) & =\eta E(s) \\
E(m) & =E(s)
\end{aligned}
$$

Nur die zweite Gleichung dieses Systems bedarf eines kurzen Kommentars. Wenn der Stpfl. einmal erfolgreich war, wird er einmal ehrlich: Der laufende Ertrag der Steuerhinterziehung ist gleich null. Dafür gelangt der Stpfl. mit Sicherheit in Zustand $s$. Die Auflösung nach $E(s)$ ergibt

$$
E(s)=\frac{q-p \alpha}{1-p \eta-q \eta^{2}}=\frac{q-p \alpha}{(1-\eta)(1+q \eta)}>0
$$

Eine innere Lösung bezüglich irgendeiner der Variablen $S_{y}$ kommt auch für den periodischen Hinterzieher nicht in Frage. $E(s)$ sinkt, wenn der Stpfl. in $s$ und $m$ weniger als $1 \mathrm{DM}$ hinterzieht. Würde er in diesen Zuständen immer nur 1/2 DM hinterziehen, halbierte sich $E(s)$.

Von allen möglichen Politiken, die der Stpfl. bei einer rückwirkenden Strafe mit einer Verjährungsfrist von einem Jahr verfolgen kann, konnten alle bis auf zwei als nicht optimal ausgeschieden werden. Die Kapitalwerte, die mit den verbleibenden zwei Politiken verbunden sind, kennen wir. Um zu entscheiden, ob $\left(S_{y}\right)^{n}$ oder $\left(S_{y}\right)^{1}$ den höchsten Ertrag liefert, vergleichen wir nun $E(s)$ und $N(s)$. Der Zähler von (6.5) ist größer als der von (6.4), da der periodische Hinterzieher keine rückwirkende Strafe zahlt. Der Nenner von (6.5) ist größer als der von (6.4), weil der periodische Hinterzieher auf den Ertrag $q-p \alpha$ eine Periode lang warten muß, wenn er einmal erfolgreich gewesen ist. Auf den ersten Blick kann also nicht 
gesagt werden, welche Politik optimal ist. Eine genauere Betrachtung der zwei Kapitalwerte ergibt:

1) Unter der Bedingung

$$
q-p \alpha-q p(1+\alpha)=1-p(1+\alpha)(1+q)<0
$$

scheidet die Politik $\left(S_{y}\right)^{n}$ aus. Sie führt zu einem negativen Kapitalwert, während die andere Politik einen positiven Ertrag liefert.

2) Gilt $1-p(1+\alpha)(1+q)>0$, kann es optimal sein, in jeder Periode zu hinterziehen, muß es aber nicht. Die Bedingung für die Wahl der Politik $\left(S_{y}\right)^{n}$ ist

$$
\begin{aligned}
& 1-p(1+\alpha)(1+q)>\frac{1-p(1+\alpha)}{1+q \eta} \\
\Leftrightarrow & 1-p(1+\alpha)\left(1+q+\eta^{-1}\right)>0
\end{aligned}
$$

Steht in (6.7) statt "größer" das "kleiner"-Zeichen, ist $\left(S_{y}\right)^{1}$ optimal.

Abbildung 6.3 zeigt alle Kombinationen von $p$ und $\alpha$, die zu einem notorischen oder periodischen Hinterzieher führen. Die Kurve $A$ grenzt zunächst den Bereich $\mathrm{ab}$, in dem die Annahme $q-p \alpha>0$ erfüllt ist. Löst man $q-p \alpha=0$ nach $\alpha$ auf, ergibt sich

$$
\alpha_{A}=\frac{1}{p}-1
$$

Nur unterhalb von $A$ wird hinterzogen. Die Kurve $B$ bildet alle Kombinationen von $p$ und $\alpha \mathrm{ab}$, die

$$
\begin{aligned}
& 1-p(1+\alpha)(1+q)=0 \\
& \Leftrightarrow \quad \alpha_{B}=\frac{1}{p(1+q)}-1
\end{aligned}
$$

erfüllen. Für $p=1$ ist $\alpha_{B}$ gleich null. Für $p<1$ gilt $\alpha_{B}>0$. B liegt für alle $p<1$ (bzw. $\alpha>0$ ) unterhalb von $A$ und ist ebenfalls konvex. Alle Punkte auf und unterhalb von $B$ sind mit Fall 1) ausgeschlossen. Die Kurve $C$ geht aus der Gleichung

$$
1-p(1+\alpha)\left(1+q+\eta^{-1}\right)=0
$$

hervor. Aufgelöst nach $\alpha$ ergibt sich

$$
\alpha_{C}=\frac{1}{p\left(1+q+\eta^{-1}\right)}-1
$$

Für eine gegebene Entdeckungswahrscheinlichkeit bestimmt diese Gleichung den Strafsatz $\alpha_{C}$, bei dem der Stpfl. indifferent zwischen den Politiken $\left(S_{y}\right)^{n}$ und $\left(S_{y}\right)^{1}$ 
Abbildung 6.3: Der notorische und der periodische Hinterzieher

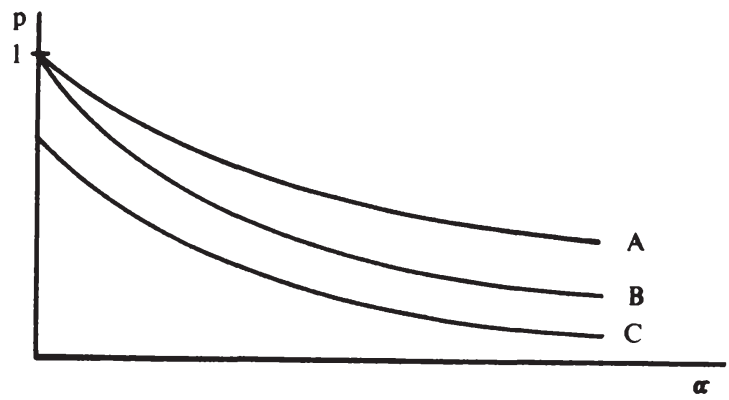

ist. $C$ liegt wegen $\eta^{-1}>0$ unterhalb von $B$, wie ein Vergleich von (6.9) und (6.8) zeigt.

Alle Kombinationen von $p$ und $\alpha$, die unterhalb von $C$ liegen, verursachen einen notorischen Hinterzieher. In dem gesamten Bereich zwischen $C$ und $A$ wird der Stpfl. einmal ehrlich, sobald er einmal erfolgreich Steuern verkürzt hat. Dies ist auch dann der Fall, wenn der Kapitalwert des notorischen Hinterziehers noch positiv ist (der Bereich zwischen $B$ und $C$ ). Der Stpfl. kann einen höheren Kapitalwert der Erträge aller Perioden erreichen, wenn er in Zustand $e$ auf den laufenden Ertrag verzichtet und die rückwirkende Strafe vermeidet. Es wäre kurzsichtig, immer nur auf den unmittelbaren Ertrag der Steuerhinterziehung in einer Periode $t$ zu schauen, statt auch die Wirkung der Hinterziehung in Periode $t$ auf die Erträge der Steuerhinterziehung in den folgenden Perioden zu beachten. Ein Modell mit wiederholter Veranlagung kann damit erklären, warum ein Stpfl. sein tatsächliches Einkommen deklariert, obwohl der Ertrag der Hinterziehung des laufenden Einkommens positiv ist. Modelle mit einmaliger Veranlagung können unter dieser Bedingung dagegen einen ehrlichen Stpfl. nicht erklären. Interessant ist noch folgende Beobachtung. Auch in dem Grenzfall $\alpha=0$ ist das kritische $p_{C}$ aus (6.9) echt kleiner als eins. Es gibt also Werte der Entdeckungswahrscheinlichkeit kleiner eins, die bewirken, daß der Stpfl. ab und an ehrlich ist, obwohl die Steuerhinterziehung strafrechtlich nicht verfolgt wird! Die Rückzahlung der in der Vergangenheit verkürzten Steuern kann eine ausreichend hohe "Strafe" sein, um den Stpfl. zu veranlassen, ehrlich zu werden, sobald er einmal erfolgreich war.

Der Diskontfaktor $\eta$ hat auf $A$ und $B$ keinen Einfluß. Die Grenze $C$ verschiebt sich jedoch nach oben, wenn $\eta$ steigt. Wird die Zukunft mit einem größeren Gewicht versehen (der durch $\eta$ implizit definierte Zinssatz sinkt), wird der Bereich, in dem immer hinterzogen wird, größer. Aus (6.6) geht schon unmittelbar hervor, 
Abbildung 6.4: Der Kapitalwert der Steuerhinterziehung in Abhängigkeit von dem Strafsatz

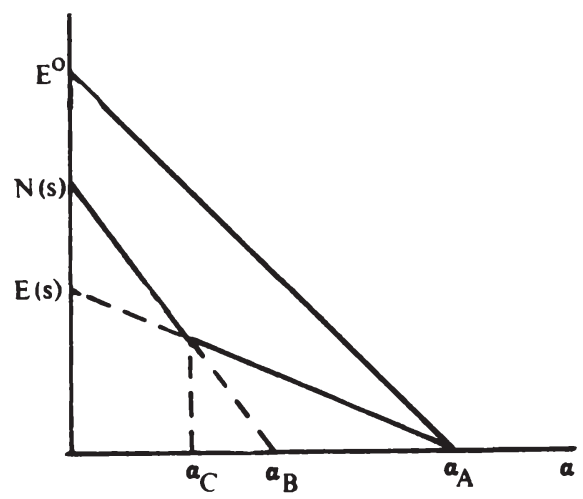

daß die Politik $\left(S_{y}\right)^{1}$ weniger attraktiv im Vergleich zu $\left(S_{y}\right)^{n}$ wird, wenn $\eta$ steigt. Der Schrecken der rückwirkenden Strafe wird mit $\eta$ nicht größer, da sie laut Annahme mit $\eta$ verzinst wird. Auf der anderen Seite erhalten die Jahre, in denen der periodische Hinterzieher in Zustand $e$ ist und ohne "Verdienst" bleibt, ein höheres Gewicht.

In Abbildung 6.3 wird ein Zusammenhang deutlich, der uns schon in dem Grundmodell der Steuerhinterziehung begegnet ist. Wenn bei konstantem Strafsatz (konstanter Entdeckungswahrscheinlichkeit) die Entdeckungswahrscheinlichkeit (der Strafsatz) ausgehend von null immer weiter erhöht wird, sinken die hinterzogenen Steuern in zwei Schritten. Zunächst wird der notorische Hinterzieher ab und an ehrlich, sobald die Grenze $C$ überschritten ist. Wenn $A$ erreicht ist, wird stets das tatsächliche Einkommen deklariert. Damit erweist sich auch in dem Modell dieses Abschnitts eine Botschaft des Grundmodells als richtig: Höhere Strafen und verschärfte Kontrollen - hier konkret die rückwirkende Kontrolle und Strafe sind wirksame Mittel gegen die Steuerhinterziehung, wenn das Einkommen exogen ist. Dies läßt sich auch mit Hilfe von Abbildung 6.4 deutlich machen, die den Kapitalwert der Steuerhinterziehung in Abhängigkeit von $\alpha$ (bei konstantem $p$ ) zeigt. $E^{0}=(q-p \alpha) /(1-\eta)$ ist der Ertrag der Steuerhinterziehung wenn keine rückwirkende Strafe verhängt wird. In Verbindung mit (6.4) und (6.5) sieht man, daß $E^{0}$ für $\alpha<\alpha_{A}$ stets größer als $E(s)$ und $N(s)$ ist. Solange also der prohibitiv hohe Strafsatz $\alpha_{A}$ keine Anwendung findet, reduziert die Einführung einer rückwirkenden Strafe - auch bei einer Verjährungsfrist von einem Jahr - den Ertrag der 
Hinterziehung bzw. den Kapitalwert aller Einkommen des Stpfl. nach Steuer und Strafe. Entsprechende Zusammenhänge ergeben sich, wenn man bei konstantem $\alpha$ den Ertrag der Hinterziehung in Abhängigkeit von $p$ abbildet.

Da der Barwert des Aufkommens aus Steuern und Strafen gleich dem Barwert der Steuern auf das tatsächliche Einkommen, 1/(1- $\eta)$, minus dem Kapitalwert aller Erträge der Steuerhinterziehung ist, wenn $\eta$ auch der Diskontfaktor des Staates ist - das soll an dieser Stelle der Einfachheit halber angenommen werden -, ergibt sich aus Abbildung 6.4 unmittelbar, daß der Barwert des Aufkommens mit der Einführung einer rückwirkenden Strafe steigt. Wenn die zusätzlichen Kosten, die eine rückwirkende Kontrolle verursacht, null sind, bietet es sich aus der Sicht der Finanzbehörden an, sie durchzuführen: Sie erbringt einen zusätzlichen Ertrag ohne zusätzliche Kosten. Daß die zusätzlichen Kosten einer rückwirkenden Kontrolle null sind, ist ein denkbarer Grenzfall: Es kann sein, daß die Erforschung der jüngsten Vergangenheit eines Stpfl. ohnehin notwendig ist um festzustellen, wie groß sein Einkommen in der gerade abgelaufenen Periode ist. Im allgemeinen wird man jedoch vermuten, daß eine rückwirkende Kontrolle Kosten verursacht. Für Finanzbehörden, die die Erträge und die Kosten ihrer Maßnahmen abwägen, stellt sich dann die Frage, ob eine Verlängerung der faktischen Verjährungsfrist die Finanzbehörden prüfen oft weniger zurückliegende Jahr als nach Steuer- und Strafrecht zulässig - netto zu einem höheren Aufkommen führt. Diese Frage wird in Abschnitt 6.2.5 aufgegriffen. In den zwei nächsten Abschnitten wird das Modells um eine beliebige Verjährungsfrist und um ein stochastisches Einkommen des Steuerhinterziehers erweitert.

\subsubsection{Der Einfluß der Verjährungsfrist auf die Steuerhin- terziehung}

Der Markov-Entscheidungsprozeß, der in den letzten beiden Abschnitten für eine Verjährungsfrist von einem Jahr dargestellt worden ist, soll nun für eine beliebige Verjährungsfrist verallgemeinert werden. Die Annahmen 2 - 9, die oben angeführt sind, werden übernommen. Ziel des Abschnitts ist es festzustellen, wie das Verhalten des Stpfl. von der Verjährungsdauer abhängt.

Bezeichne $v$ die Verjährungsfrist: Eine Steuerhinterziehung, die länger als $v$ Jahre zurückliegt, kann weder zu einer Nachzahlung noch zu einer Strafe führen. Sei $Z=\left\{s, m, e_{1}, e_{2}, \ldots e_{v}\right\}$ der Zustandsraum des Prozesses. In Zustand $s$ gelangt der Stpfl., wenn er in der letzten Periode sein tatsächliches Einkommen deklariert hat. Nach $e_{x}, x=1, \ldots, v-1$, gelangt der Stpfl., wenn er gerade $x$ mal hintereinander erfolgreich hinterzogen hat. Nach $e_{v}$ gelangt er, wenn er mindestens $v$ mal hintereinander erfolgreich Steuern verkürzt hat. In den Zustand $m$ kommt er bei einem Mißerfolg. Wenn der Stpfl. in jedem Zustand hinterzieht, ist die Matrix der 
Übergangswahrscheinlichkeiten $\left(p_{y z}\left[\left(S_{y}\right)\right]\right)$ gleich

$$
\left.\begin{array}{l|ccccccccc} 
& s & m & e_{1} & e_{2} & e_{3} & \cdots & e_{v-2} & e_{v-1} & e_{v} \\
s & 0 & p & q & - & - & & - & - & - \\
m & 0 & p & q & - & - & & - & - & - \\
e_{1} & 0 & p & - & q & - & & - & - & - \\
e_{2} & 0 & p & - & - & q & & - & - & - \\
\vdots & & & & & & & & & \\
e_{v-3} & 0 & p & - & - & - & & q & - & - \\
e_{v-2} & 0 & p & - & - & - & & - & q & - \\
e_{v-1} & 0 & p & - & - & - & & - & - & q \\
e_{v} & 0 & p & - & - & - & & - & - & q
\end{array}\right)
$$

Der Stpfl. kann jedoch in jeder Zeile dieser Matrix $q$ und $p$ durch null und die Null am Anfang der Zeile durch eine Eins ersetzen, indem er in dem betreffenden Zustand sein tatsächliches Einkommen deklariert. Ein Querstrich steht dort, wo kein Übergang möglich ist.

Der Übergang in den Zustand $s$ bringt keinen (laufenden) Ertrag. Bei einem Übergang in einen der Zustände $e_{1}, e_{2}, \ldots e_{v}$ ist der Ertrag $1 \mathrm{DM}$. Der Übergang von $e_{x}, x \leqq v$, nach $m$ führt zu der Strafe

$$
-\alpha-(1+\alpha) \sum_{i=1}^{x} \eta^{-i}
$$

Die rückwirkende Strafe ist zu verzinsen. Die Strafe wird durch die Verjährung begrenzt: Maximal $v$ zurückliegende Jahre können bei der Bemessung der Geldstrafe neben der laufenden Periode berücksichtigt werden ${ }^{3}$.

Für den erwarteten Ertrag der Steuerhinterziehung in den Zuständen $s$ (und $m)$ und $e_{1}, e_{2}, \ldots e_{v}$ werden die folgenden Abkürzungen verwendet:

$$
\begin{array}{ll}
s: & h_{0}=1-p(1+\alpha) \\
e_{1}: & h_{1}=1-p(1+\alpha)\left(1+\eta^{-1}\right) \\
e_{2}: & h_{2}=1-p(1+\alpha)\left(1+\eta^{-1}+\eta^{-2}\right) \\
\cdots & \cdots=\cdots \cdots \\
e_{x}: & h_{x}=1-p(1+\alpha) \sum_{i=0}^{x} \eta^{-i}
\end{array}
$$

$1+\alpha$ wird im folgenden mit $\pi$ abgekürzt.

\footnotetext{
${ }^{3}$ Eine Strafe mit einer Rückwirkung über mehrere Perioden kann schnell das laufende Einkommen des Stpfl. übersteigen. Da er unendlich lange lebt, kann die Finanzbehörde fällige Zahlungen jedoch gegen Zinsen stunden (vgl. §§ 222 und 234 AO). Der Barwert der Strafe ist bei einer Verzinsung mit $\eta$ unabhängig davon, wann sie bezahlt wird.
} 
Aus der Menge aller Politiken, die der Stpfl. verfolgen kann, kommt nur ein Bruchteil in die engere Auswahl, da die optimale Politik stationär ist und man bestimmte Politiken, die von anderen dominiert werden, schnell identifizieren kann. Die $v+1$ Politiken, die übrigen bleiben, sind:

$$
\begin{aligned}
\left(S_{y}\right)^{1} & =(110) \\
\left(S_{y}\right)^{2} & =(1110) \\
\left(S_{y}\right)^{3} & =(11110) \\
\cdots & =\cdots \cdots \\
\left(S_{y}\right)^{v-1} & =(11111 \cdots 10) \\
\left(S_{y}\right)^{v} & =(11111 \cdots 110) \\
\left(S_{y}\right)^{n} & =(11111 \cdots 111)
\end{aligned}
$$

Der Stpfl. wird in einem der Zustände $e_{x}, x \leqq v$, ehrlich oder er ist ein notorischer Hinterzieher. Wenn er in $e_{x}$ sein tatsächliches Einkommen angibt, kann er in die Zustände $e_{x+j}, j \geqq 1$, nicht übergehen, da er wieder in $s$ startet. Er muß sich folglich nicht überlegen, was er in diesen Zuständen tut. Die Vektoren, die für jeden Zustand die Entscheidung des Stpfl. angeben, sind entsprechend verkürzt. Die diskontierten Erträge aller Perioden, die aus den angeführten Entscheidungen resultieren, werden mit $E^{x}(s), x=1, \ldots, v$, und $N^{v}(s)$ bezeichnet. $E^{x}(s)$ ist der Kapitalwert der Steuerhinterziehung, wenn der Stpfl. einmal ehrlich wird, sobald er gerade $x$ mal hintereinander Erfolg hatte. $N^{v}(s)$ ist der diskontierte Ertrag eines notorischen Hinterziehers, wenn die Verjährungsfrist $v$ Jahre beträgt. Diese Erträge können mit Hilfe der entsprechenden Matrix der Übergangswahrscheinlichkeiten und den laufenden Erträgen, die bei den einzelnen Übergängen anfallen, nach dem oben vorgeführten Verfahren berechnet werden. Es ist jeweils ein lineares Gleichungssystem aufzustellen und nach dem Kapitalwert der Steuerhinterziehung in Zustand $s$ aufzulösen.

$N^{1}(s)$ ist oben bereits berechnet worden:

$$
N^{1}(s)=\frac{1-p \pi(1+q)}{1-\eta}
$$

Weiter gilt

$$
N^{2}(s)=\frac{1-p \pi\left(1+q+q^{2}\right)}{1-\eta}
$$

Daraus sehen wir bereits, daß man für eine beliebige endliche Verjährungsfrist folgenden Ausdruck erhält:

$$
N^{\nu}(s)=\frac{1-p \pi \sum_{i=0}^{\nu} q^{i}}{1-\eta}
$$




$$
\begin{aligned}
& =\frac{1-p \pi\left(1-q^{v+1}\right) /(1-q)}{1-\eta} \\
& =\frac{1-(1+\alpha)\left(1-q^{v+1}\right)}{1-\eta}
\end{aligned}
$$

Betrachten wir den Zähler von $N^{v}$ in der ersten Zeile. Verkürzt der Stpfl. seine Steuerschuld in Periode $t$ um $1 \mathrm{DM}$, muß er $v+1$ mal damit rechnen, daB er "erwischt" wird und $\pi \mathrm{DM}$ an die Finanzbehörde zahlen muß. Erst mit Ablauf der Verjährungsfrist - im Jahr $t+v+1-$ muß er nicht mehr damit rechnen, für die Hinterziehung in Periode $t$ belangt zu werden. Da der notorische Hinterzieher nie ehrlich ist, gibt es keine andere Grenze für die Strafe. Der betrachtete Zähler ist der Ertrag, der der Steuerhinterziehung in jeder Periode zuzurechnen ist. Daher hat der Nenner von $N^{v}(s)$ eine besonders einfache Form.

Wie groß ist der Kapitalwert, wenn der Stpfl. in den Zuständen $e_{x}, x \leqq v$, ehrlich wird? $E^{1}(s)$ ist bereits bekannt:

$$
E^{1}(s)=\frac{h_{0}}{(1-\eta)(1+q \eta)}
$$

Die zwei nächsten Erträge sind:

$$
\begin{gathered}
E^{2}(s)=\frac{h_{0}+h_{1} q \eta}{(1-\eta)\left(1+q \eta+q^{2} \eta^{2}\right)} \\
E^{3}(s)=\frac{h_{0}+h_{1} q \eta+h_{2} q^{2} \eta^{2}}{(1-\eta)\left(1+q \eta+q^{2} \eta^{2}+q^{3} \eta^{3}\right)}
\end{gathered}
$$

Daraus folgere ich für den Ertrag bei einem "Stop" der Hinterziehung in Zustand $e_{x}$ :

$$
E^{x}(s)=\frac{\sum_{i=0}^{x-1} h_{i}(q \eta)^{i}}{(1-\eta) \sum_{i=0}^{x}(q \eta)^{i}}
$$

Die Erträge aller in Frage kommenden Politiken sind nun bekannt. Die optimale Politik ist gerade die, die den höchsten Ertrag liefert. Leider ist damit nichts über die Eigenschaften der optimalen Politik gesagt. Eine wichtige Eigenschaft der besten Politik wird erst deutlich, wenn man fragt, wie das Verhalten des Stpfl. von der Verjährungsfrist abhängt. Zu diesem Zweck wird zunächst beschrieben, wie $N^{v}$ von $v$ abhängt ${ }^{4}$. Dann wird gezeigt, wie $E^{x}$ von $x$ abhängt. Anschließend werden beide Überlegungen zusammengenommen und gezeigt, wie sich die optimale Politik mit einer Verlängerung der Verjährungsfrist verändert.

\footnotetext{
${ }^{4}$ Alle Erwartungswerte werden im folgenden auf den Zustand $s$ bezogen.
} 
Abbildung 6.5: Der Ertrag des notorischen Hinterziehers in Abhängigkeit von der Verjährungsfrist

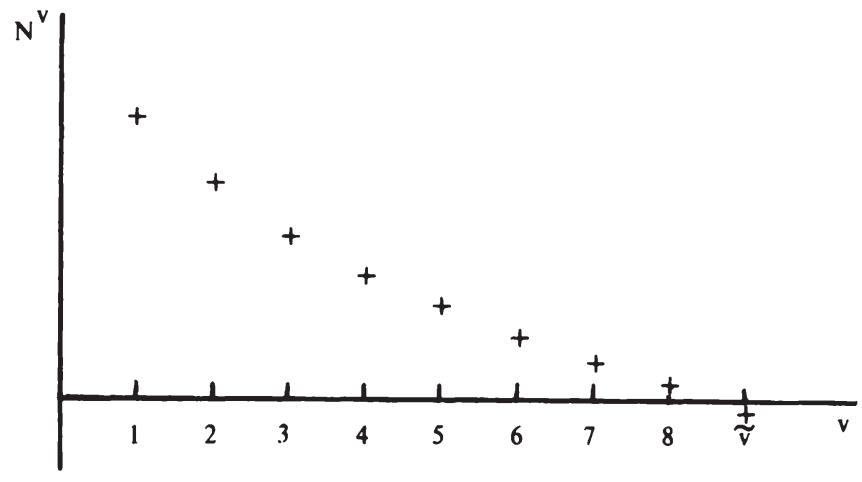

Der Kapitalwert $N^{v}$ ist am größten, wenn es keine rückwirkende Kontrolle gibt $(v=0)$ :

$$
N^{0}=\frac{1-p \pi}{1-\eta}
$$

Ausgehend von $N^{0} \operatorname{sinkt} N^{v}$ mit $v$ :

$$
\Delta N^{v}=N^{v+1}-N^{v}=\frac{\pi q^{v+1}(q-1)}{1-\eta}<0
$$

Die "zweite Ableitung" ist

$$
\Delta N^{v+1}-\Delta N^{v}=\frac{\pi q^{v+1}(q-1)^{2}}{1-\eta}>0
$$

Der Ertrag des notorischen Hinterziehers sinkt mit abnehmender Rate, wenn die Verjährungsfrist steigt. Schließlich wird ein $\tilde{v} \geqq 1$ erreicht, bei dem $N^{v}$ erstmals negativ wird. Der Grenzwert von $N^{v}$ ist bei immer größer werdendem $v$ gleich $-\alpha /(1-\eta)$ : Die Steuerhinterziehung jeder Periode wird irgendwann entdeckt und bestraft. Qualitativ hat $N^{v}$ damit den in Abbildung 6.5 gezeigten Verlauf.

Wie hängt $E^{x}$ von $x$ ab? Den (positiven) Ertrag $E^{x}$ für den kleinsten Wert von $x(x=1)$ kennen wir bereits. Lohnt es sich, später als in Zustand $e_{1}$ ehrlich zu werden? Sei $\Delta E^{x}=E^{x+1}-E^{x}$. Ist diese Differenz positiv, ist es vorteilhaft, 
Abbildung 6.6: Der Ertrag des Hinterziehers, der nach $x$ Erfolgen einmal ehrlich wird

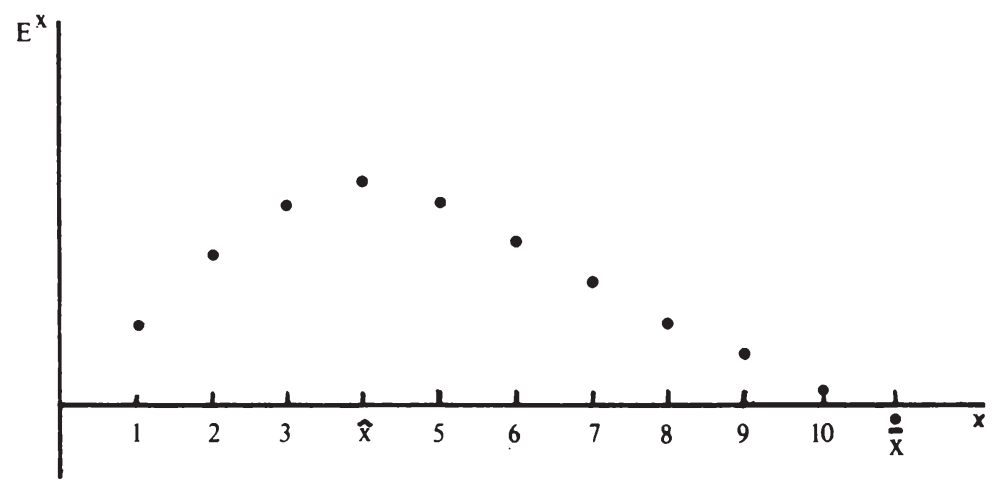

statt in $e_{x}$ erst in Zustand $e_{x+1}$ einmal ehrlich zu werden. Anhang 6.2.3 a zeigt:

$$
\Delta E^{x}=\frac{(q \eta)^{x}\left[1-\left\{p \pi \sum_{i=0}^{x}\left(\eta^{-i} \sum_{j=0}^{i}(q \eta)^{j}\right)\right\}\right]}{(1-\eta) \sum_{i=0}^{x+1}(q \eta)^{i} \sum_{i=0}^{x}(q \eta)^{i}}
$$

Der Nenner von $\Delta E^{x}$ ist positiv. Der Zähler kann für kleine $x$ positiv sein. Es gibt jedoch ein $\widehat{x}$, für das der Zähler erstmals negativ wird, da der Ausdruck in der geschweiften Klammer mit $x$ wächst. Für die "zweite Ableitung" von $E^{x}$, $\Delta E^{x+1}-\Delta E^{x}$, zeigt Anhang 6.2.3 a: Sie ist kleiner null, solange $\Delta E^{x} \geqq 0$ ist. Wenn $\Delta E^{x}<0$ ist, kommt ein Punkt, an dem $\Delta E^{x+1}-\Delta E^{x}$ das Vorzeichen wechselt. Diese zweite Differenz ist dann nicht mehr kleiner null, sondern größer null. Aus (6.10) ergibt sich: Es gibt ein $\bar{x}$, bei dem $E^{x}$ erstmals negativ wird. Schließlich zeigt Anhang 6.2.3 b: Wenn der Stpfl. erst nach sehr vielen Erfolgen einmal ehrlich wird, hat er in etwa denselben Ertrag wie ein notorischer Hinterzieher, da eine sehr lange Erfolgsserie nicht sehr wahrscheinlich ist.

$$
\lim _{x \rightarrow \infty} E^{x}=-\frac{\alpha}{1-\eta}
$$

Einen möglichen Verlauf von $E^{x}$ zeigt Abbildung 6.6. Unter der Annahme, daß die Verjährungsfrist $v$ größer als alle interessanten Abbruchszeitpunkte $x$ ist, 
steigt der Kapitalwert des Stpfl. zunächst, wenn er erst nach einer längeren Erfolgsserie ehrlich wird. Diese Aussage unterstellt, daß $\alpha$ und/oder $p$ genügend klein sind, da sonst schon - anders als in der Abbildung $-E^{2}<E^{1}$ gelten könnte. Schließlich kommt ein Zustand $e_{\hat{x}}$, in dem es sich nicht mehr lohnt, noch einmal das Einkommen zu hinterziehen, falls in der letzten Periode wieder keine Kontrolle stattgefunden hat. Die rückwirkende Strafe ist dann so groß geworden, daß der Stpfl. lieber einmal ehrlich ist und auf diese Weise dafür sorgt, daß seine Vergangenheit "verjährt" ist - das Finanzamt bricht ja die rūckwirkende Kontrolle ab, sobald diese in einer Periode kein Mehrergebnis liefert. Wenn die Verjährungsfrist also nur groß genug ist, erhalten wir das Ergebnis von Allingham/Sandmo (1972, S. 336 f.): Rückwirkende Kontrollen führen dazu, daß es Perioden gibt, in denen die Steuerhinterzieher ehrlich werden.

Bevor die Abbildungen 6.5 und 6.6 zusammengefügt werden können und gezeigt werden kann, wie die Politik des Stpfl. von der Verjährungsfrist abhängt, werden noch zwei Aussagen benötigt, deren Ableitung Anhang 6.2.3 c enthält. Es gilt die Äquivalenz

$$
\text { (i) } \Delta E^{x} \gtreqless 0 \Leftrightarrow E^{x} \lesseqgtr N^{x}
$$

Solange der Ertrag der Hinterziehung steigt (sinkt), wenn der Stpfl. in $e_{x+1}$ statt in $e_{x}$ ehrlich wird, ist sein Ertrag größer (kleiner), wenn die Verjährungsfrist $v$ gerade $x$ Jahre beträgt und der Stpfl. notorisch hinterzieht. Ferner gilt

$$
\text { (ii) } E^{x} \lesseqgtr N^{x} \Leftrightarrow E^{x+1} \lesseqgtr N^{x}
$$

wobei $v$ wieder durch $x$ ersetzt worden ist.

Setzt man unter Berücksichtigung von (i) und (ii) die Abbildungen 6.5 und 6.6 zusammen, ergibt sich die in Abbildung 6.7 gezeigte Konstellation. Solange $\Delta E^{x}$ positiv ist, ist $N^{x}$ größer als $E^{x}$ (und $E^{x+1}$ ). Sobald $\Delta E^{x}$ negativ wird, ist $N^{x}$ kleiner als $E^{x}$ (und $E^{x+1}$ ).

Wie ändert der Stpfl. seine Politik, wenn die Verjährungsfrist angefangen von eins sukzessive erhöht wird? Beträgt die Verjährungsfrist $v$ Jahre, müssen alle Erträge $E^{x}$ mit $x>v$ und alle $N^{x}$ mit $x \neq v$ vernachlässigt werden. Von den verbleibenden Kapitalwerten kann dann unmittelbar der höchste Wert angegeben werden. Mit $v=1$ ist $N^{1}>E^{1}$ der höchste Ertrag. Für $v=2$ finden wir $N^{2}>E^{2}>E^{1}$. Auch für $v=3$ bleibt der Stpfl. noch ein notorischer Hinterzieher. Die Verlängerung der Verjährungsfrist auf vier Jahre führt dazu, daß der Stpfl. nach einer Serie von vier Erfolgen einmal steuerehrlich wird: $E^{4}$ ist größer als $N^{4}$. Jede weitere Verlängerung der Verjährungsfrist ändert das Verhalten des Stpfl. und seinen Ertrag nicht. Für alle Verjährungsfristen $v \geqq 4$ sorgt er nach vier Erfolgen dafür, seine Vergangenheit vergessen zu machen, statt die gesetzliche Verjährung abzuwarten. 
Abbildung 6.7: Die Erträge des notorischen Hinterziehers und des nach $x$ Erfolgen ehrlichen Stpfl.

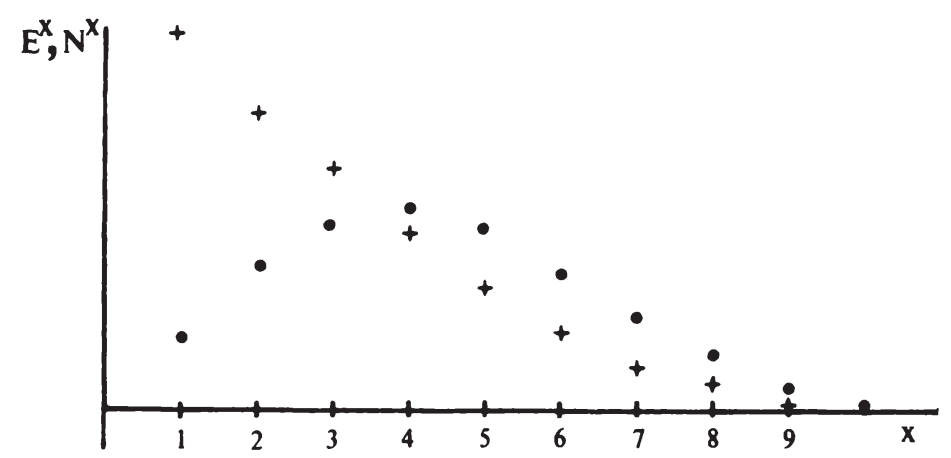

Der Einfluß der Verjährungsfrist auf die Steuerhinterziehung läßt sich allgemein wie folgt beschreiben. Wird die Frist, nach der die Vergangenheit des Steuerhinterziehers keinen Einfluß mehr auf seine heutige Strafe hat, angefangen von einem Jahr sukzessive erhöht, sinkt der diskontierte Ertrag aller Perioden zunächst, da die rückwirkende Strafe immer größer wird; der Stpfl. bleibt jedoch ein notorischer Hinterzieher (sofern $\alpha$ und $p$ nicht zu groß sind). Schließlich ist die Verjährungsfrist so lang geworden, daß es vorteilhafter ist, nach einer bestimmten Anzahl von Erfolgen einmal steuerehrlich zu sein, um die rückwirkende Strafe zu vermeiden. Eine weitere Verlängerung der Verjährungsfrist ändert das Verhalten des Stpfl. und den diskontierten Ertrag der Steuerhinterziehung nicht mehr. Der Stpfl. sorgt selbst für eine "Verjährung" seiner Taten. Die Verlängerung der Verjährungsfrist - eine Form verschärfter Kontrollen und Strafen - ist also zunächst ein wirksames Mittel gegen die Steuerhinterziehung, da sie den Ertrag der Hinterziehung senkt und das erwartete Aufkommen steigert. Da die Finanzbehörde jedoch die rückwirkende Kontrolle beendet, sobald in einer Periode das tatsächliche Einkommen deklariert worden ist, ist eine längere Verjährungsfrist $\mathrm{ab}$ einem bestimmten Punkt nicht weiter in der Lage, das erwartete Aufkommen zu erhöhen.

Am Schluß dieses Abschnitts vergleiche ich die vorangehende Analyse mit dem "dynamic case" von Allingham/Sandmo (1972). Bei Allingham/Sandmo gibt es keine Verjährung, einen risikoaversen Stpfl. und eine Strafe, die von dem hinterzogenen Einkommen abhängt und nicht verzinst wird. Da die Verjährung der zentrale Unterschied ist, gehe ich für den Vergleich von $v \rightarrow \infty$ aus. Allingham/Sandmo 
unterscheiden zwei Typen von Hinterziehern: Ein "myopic individual" sieht nur, daß die heute zu erwartende Strafe von seiner Vergangenheit abhängt. Es kennt mit anderen Worten den Zustand, in dem es sich befindet, und maximiert den Erwartungsnutzen des Konsums des laufenden Einkommens nach Steuer und Strafe. Es sieht nicht, $d a ß$ " $a$ decision to cheat today involves mortaging the future, since the stochastic penalty is in effect delayed" (Allingham/Sandmo, 1972, S. 334). Ein "consistent individual" sieht dagegen neben der Vergangenheit die durch seine heutige Entscheidung beeinflußte Zukunft.

Allingham/Sandmo (1972, S. 335 f.) zeigen, daß der myopische Stpfl. von Periode zu Periode weniger hinterzieht, solange er nicht geprüft wird, und schließlich sein tatsächliches Einkommen deklariert. Es lohnt sich immer weniger, das laufende Einkommen zu hinterziehen, da die durch die Vergangenheit festgelegte rückwirkende Strafe mit der Länge einer Erfogsserie steigt. Dieses Ergebnis erhält man mit einem Unterschied auch mit der in diesem Kapitel verwendeten Strafe: Solange

$$
h_{x}=1-p \pi \sum_{i=0}^{x} \eta^{-i}
$$

positiv ist, hinterzieht der myopische Stpfl. - und zwar infolge der Risikoneutralität jeweils das gesamte Einkommen. Sobald $h_{x}$ erstmals negativ wird, wird er ehrlich.

Im Anschluß an den myopischen Stpfl. untersuchen Allingham/Sandmo (1972, S. 336 f.), in wieweit sich ein konsistenter Stpfl. anders als jener verhält. Ihre "intuitive ideas" sind: (i) Auch ein konsistenter Stpfl. bricht eine Erfolgsserie schließlich ab. (ii) "the consistent individual will always declare more than the myopic individual: it is then indeed 'short-sighted to evade taxes' ". Die vorangehende Analyse stützt beide Punkte. $\Delta E^{x}$ wird ab einem bestimmten $x$ negativ. Eine Fortsetzung der Hinterziehung lohnt sich dann nicht mehr. Vergleicht man den Zähler von $\Delta E^{x}$ und $h_{x}$, folgt: $\Delta E^{x}$ wird eher negativ als $h_{x}$, wenn $p$ und $\pi$ nicht zu groß sind. Der konsistente Stpfl. wird eine Erfogsserie eher als der myopische Stpfl. unterbrechen. Er deklariert also über alle Perioden gerechnet mehr - auch wenn er in bestimmten Zuständen infolge der Risikoneutralität genau wie der myopische Stpfl. sein gesamtes Einkommen hinterzieht.

\subsubsection{Stochastisches Einkommen}

Wenn der Stpfl. ein festes Einkommen hat, ist es auf die Dauer nicht plausibel, daß er in jeder Periode mit einer gegebenen Wahrscheinlichkeit $p<1$ geprüft wird, wenn man gleichzeitig sehr viele Veranlagungsperioden annimmt. Nach einigen Prüfungen "kennt" die Finanzbehörde das feste Einkommen $Y$. Sie könnte dann auf die naheliegende Idee kommen, den Stpfl. nur dann zu prüfen, wenn er nicht genau $Y$ deklariert. Das Modell gewinnt an Plausibilităt, wenn man das feste 
durch ein zufälliges Einkommen ersetzt ${ }^{5}$. Dies soll in diesem Abschnitt für eine sehr einfache Verteilung geschehen. Einen komplizierteren Fall behandele ich nicht, da schon die Analyse dieses einfachen Falls und die Arbeit von Landsberger/Meilijson (1982) zu der Vermutung berechtigen, daß die Einführung eines stochastischen Einkommens den Kern der dynamischen Modelle nicht berührt (siehe dazu auch Abschnitt 6.4).

Das zu versteuernde Einkommen $Y$ des Stpfl. nimmt in jeder Periode mit der Wahrscheinlichkeit $h$ den Wert $Y^{h}$ und mit der Wahrscheinlichkeit $l=1-h$ den Wert 0 an. Die Realisation seines Einkommens in der laufenden Periode kennt der Stpfl. im Gegensatz zu dem Finanzamt, wenn er seine Steuererklärung ausfüllt. Laut Tarif resultiert aus dem Einkommen $Y^{h}$ eine Steuerschuld von 1 DM. Mit einem Einkommen von 0 entsteht keine Steuerschuld. Daher wird der Stpfl. in diesem Fall sein Einkommen wahrheitsgemäß angeben. Mit dem hohen Einkommen $Y^{h}$ besteht jedoch die Versuchung, 1 DM Steuern zu hinterziehen.

Die Verjährungsfrist betrage ein Jahr. Statt der drei Zustände gibt es mit einem zweipunktverteilten Einkommen sechs Zustände $s^{j}, m^{j}, e^{j}, j=l, h$, da der Stpfl. in jedem Zustand ein niedriges oder ein hohes Einkommen haben kann. Die Zustände geben neben der steuerlich relevanten Vergangenheit auch das Einkommen der laufenden Periode an. In den Zuständen, die mit einem $l$ indiziert sind, ist der Stpfl. ehrlich, so daß er in der nächsten Periode in $s^{l}$ oder in $s^{h}$ landet. In $s^{h}$ und $m^{h}$ lohnt sich die Steuerhinterziehung auf jeden Fall. In $e^{h}$ stellt sich der Stpfl. wieder die Frage, ob er nach einem Erfolg einmal sein tatsächliches Einkommen deklarieren sollte. Je nach der Antwort auf diese Frage sind zwei Politiken zu unterscheiden. Die Übergangswahrscheinlichkeiten, die aus ihnen resultieren, enthält die folgende Matrix. In der sechsten Zeile gelten die Wahrscheinlichkeiten in den runden für den periodischen und die Wahrscheinlichkeiten in den eckigen Klammern für den notorischen Hinterzieher. Die Wahrscheinlichkeiten in den Klammern des jeweils anderen Typs sind null. Die nicht eingeklammerten Wahrscheinlichkeiten haben für beide Typen Gültigkeit. Die Erträge, die bei den Übergängen von einem Zustand in den anderen anfallen, entsprechen den in Abschnitt 6.2.1 angegebenen.

\footnotetext{
${ }^{5}$ Wenn man $p$ mit Reinganum/Wilde (1986, S. 742) als die Wahrscheinlichkeit interpretiert, mit der es der Finanzbehörde gelingt, gerichtsverwertbare Beweise für die Steuerhinterziehung zu finden, obwohl sie selbst "weiß", daß der Stpfl. nicht ehrlich ist, sind auch die Modelle mit exogenem Einkommen plausibel.
} 


\begin{tabular}{|c|c|c|c|c|c|c|}
\hline & $s^{l}$ & $s^{h}$ & $m^{l}$ & $m^{h}$ & $e^{l}$ & $e^{h}$ \\
\hline$s^{l}$ & $l$ & $h$ & 0 & 0 & 0 & 0 \\
\hline$s^{h}$ & 0 & 0 & $l p$ & $h p$ & $l q$ & $h q$ \\
\hline$m^{l}$ & $l$ & $h$ & 0 & 0 & 0 & 0 \\
\hline$m^{h}$ & 0 & 0 & $l p$ & $h p$ & $l q$ & $h q$ \\
\hline$e^{l}$ & $l$ & $h$ & 0 & 0 & 0 & 0 \\
\hline$e^{h}$ & $(l)$ & (h) & {$[l p]$} & {$[h p]$} & {$[l q]$} & {$[h q]$} \\
\hline
\end{tabular}

Ich lasse den Stpfl. in Zustand $s^{h}$ starten. Die Kapitalwerte aller Erträge eines notorischen und eines periodischen Hinterziehers $\left(N\left(s^{h}\right)\right.$ und $\left.E\left(s^{h}\right)\right)$ werden aus den zwei entsprechenden Gleichungssystemen berechnet. Dieses lautet für den zuletzt genannten Fall

$$
\begin{gathered}
E\left(s^{h}\right)=E\left(m^{h}\right)=q-p \alpha+q \eta\left[h E\left(e^{h}\right)+l E\left(e^{l}\right)\right]+p \eta\left[h E\left(m^{h}\right)+l E\left(m^{l}\right)\right] \\
E\left(s^{l}\right)=E\left(m^{l}\right)=E\left(e^{l}\right)=E\left(e^{h}\right)=\eta\left[h E\left(s^{h}\right)+l E\left(s^{l}\right)\right]
\end{gathered}
$$

In den Zuständen $s^{h}$ und $m^{h}$ erzielt der Stpfl. einen laufenden Ertrag $q-p \alpha$. Anschließend geht er in einen von vier Zuständen über. In den Zuständen mit einem Index $l$ ist der Stpfl. ehrlich und wartet darauf, in der nächsten Periode nach $s^{h}$ zu kommen. Das gleiche gilt für den Zustand $e^{h}$.

Löst man das Gleichungssystem nach dem Kapitalwert am "Start" in $s^{h}$ auf, ergibt sich

$$
E\left(s^{h}\right)=\frac{(q-p \alpha)(1-\eta(1-h))}{(1-\eta)(1+h \eta q)}
$$

Ich verzichte darauf, das Gleichungssystem wiederzugeben, mit dem der Kapitalwert des notorischen Hinterziehers

$$
N\left(s^{h}\right)=\frac{[q-p \alpha-q p h(1+\alpha)](1-\eta(1-h))}{1-\eta}
$$

berechnet werden kann. Eine Kontrolle der Richtigkeit der Rechnung erhält man, indem man $h=1$ setzt: Es ergeben sich die zwei aus Abschnitt 6.2.2 bekannten Kapitalwerte für den Fall eines festen Einkommens.

Unter welcher Bedingung wird der Stpfl. in Zustand $e^{h}$ steuerehrlich? $E\left(s^{h}\right)$ ist größer als $N\left(s^{h}\right)$, wenn

$$
1-p(1+\alpha)\left(1+q h+\eta^{-1}\right)<0
$$

gilt. Für $h=1$ kennen wir diese Bedingung bereits.

Ich breche die Analyse des Falls, daB der Stpfl. ein stochastisches Einkommen mit den Realisationen 0 und $Y^{h}$ hat, an dieser Stelle ab. Die Botschaft des dynamischen Modells mit rückwirkenden Kontrollen wird durch die Einführung eines 
zufälligen Einkommens nicht berührt: Der Stpfl. hat einen Anreiz, das deklarierte Einkommen in Abhängigkeit von seiner steuerlich relevanten Vergangenheit zu variieren. Die Realisation seines Einkommens ist eine zusätzliche Determinante seiner Entscheidung. Ist es sehr niedrig, lohnt sich seine Hinterziehung nicht. Mit einem hohen Einkommen besteht dagegen der Anreiz, die Steuer zu verkürzen, wenn die rückwirkende Strafe nicht zu schwer wiegt.

Nehmen wir jetzt an, das Einkommen $Y$ sei wie folgt verteilt:

$$
Y= \begin{cases}Y^{h}>Y^{l} & \text { mit der Wahrscheinlichkeit } h \\ Y^{l}>0 & \text { mit der Wahrscheinlichkeit } l=1-h\end{cases}
$$

Wenn auch das niedrige Einkommen positiv ist, wird der Stpfl. es möglicherweise ebenfalls nicht deklarieren. Nach einigen Prüfungen vermutet die Finanzbehörde zu Recht, daß der Stpfl. mindestens $Y^{l}$ verdient. Sie kann daher die gegebene Kontrollwahrscheinlichkeit $p$ durch folgende Regel ersetzen: Deklariert der Stpfl. nicht mindestens $Y^{l}$, wird er mit einer Wahrscheinlichkeit geprüft, die gleich $1 /(1+$ $\alpha)$ ist. Wenn er mindestens $Y^{l}$ angibt, wird er wie gehabt mit $p<1 /(1+\alpha)$ kontrolliert. Der Stpfl. wird nach dieser Änderung der Spielregeln nur den Teil $Y^{h}-Y^{l}$ seines hohen Einkommens hinterziehen - womit wir wieder bei dem zuerst analysierten Fall sind.

Lernt die Finanzbehörde in der gerade beschriebenen Weise aus den Prüfungen vergangener Perioden, ergibt sich eine interessante Beobachtung: Bei gleichem erwarteten Einkommen $h Y^{h}+l Y^{l}$ (und gleichem $h$ ) profitiert der Stpfl. mit der größeren Variation des Einkommens (gemessen an der Differenz $Y^{h}-Y^{l}$ ) langfristig in höherem Maße von den sich bietenden Möglichkeiten zur Steuerhinterziehung als ein Stpfl., dessen Einkommen weniger stark schwankt. Denn mit der Differenz der Steuern $T\left(Y^{h}\right)-T\left(Y^{l}\right)$, die mit $Y^{h}-Y^{l}$ steigt, müssen die Kapitalwerte multipliziert werden, die oben für den speziellen Fall $T\left(Y^{h}\right)-T\left(Y^{l}\right)=1$ angegeben worden sind.

\subsubsection{Der Nettoeffekt rückwirkender Kontrollen}

Lohnt es sich aus der Sicht von Finanzbehörden, die mit ihrem Budget ein möglichst großes Steueraufkommen erzielen wollen, rückwirkende Kontrollen einzuführen? Wenn die rückwirkenden Kontrollen keine zusätzlichen Kosten verursachen, lohnt sich ihre Einführung, da sie den erwarteten Ertrag der Steuerhinterziehung senken. Bei exogenem Einkommen heißt das ja, daß das Steueraufkommen steigt. Verursacht die rückwirkende Kontrolle zusätzliche Kosten, stellt sich die Frage nach dem Nettoeffekt. Dieser Abschnitt untersucht, ob bei gleichen Kosten der Steuerfahndung 
- das statische System der Sicherung des Steuereingangs, in dem nur die letzte Steuererklärung mit der immer gleichen Wahrscheinlichkeit $p_{s}$ geprüft wird,

- oder das dynamische System einer rückwirkenden Kontrolle mit einer Verjährungsfrist von einem Jahr

zu einem höheren Steueraufkommen führt.

Pro Kopf der steuerpflichtigen Bevölkerung, deren Umfang über die Zeit konstant bleibe, verfügt die Finanzbehörde über ein Budget $K$. Die Prüfung der letzten Steuererklärung jedes (identischen) Stpfl. kostet unabhängig von dem Umfang der Hinterziehung $k$. Wird die vorletzte Erklärung gleich mit geprüft, fallen zusätzliche Kosten $\theta k$ an $(\theta>0)$. Bei statischen Kontrollen können in jeder Periode $p_{s}=K / k \%$ der Bevölkerung geprüft werden. Es gelte $p_{s}<1 / \pi=1 /(1+\alpha)$, da sonst nicht hinterzogen wird. Der Steuerausfall pro Kopf und pro Periode beträgt $1-p_{s} \pi$.

Wieviel Stpfl. können bei dynamischen Kontrollen mit einem Budget $K$ geprüft werden, und wie groß ist der entsprechende Aufkommensverlust? Die Antwort auf diese Fragen setzt eine Annahme und eine Vorüberlegung voraus. Die - provisorische - Annahme lautet, daß alle Stpfl. notorische Hinterzieher sind. Unter dieser Voraussetzung sind eine Periode nach dem Start in Zustand $s$ alle Stpfl. in den Zuständen $m$ und $e$. Nach $s$ kehren sie nicht mehr zurück. Langfristig sind $g_{e} \%$ der Stpfl. in $e$ und $g_{m}=1-g_{e} \%$ in $m$, wobei $g_{e}$ sich aus der Gleichung

$$
p g_{e}=q\left(1-g_{e}\right)
$$

bestimmt. $p \%$ der Stpfl. in Zustand $e$ wandern in jeder Periode nach $m . q \%$ der Mitglieder von $m$ gelangen nach $e$, so daß sich $\mathrm{Zu}$ - und Abgänge saldieren. Man kann diesen Zusammenhang auch anders ausdrücken: Die Wahrscheinlichkeit, einen betimmten Stpfl. in $e$ anzutreffen, nachdem seit dem Start des Prozesses $t$ Perioden vergangen sind, konvergiert mit $t \rightarrow \infty$ gegen $g_{e}$. Entsprechend ist $g_{m}$ die stationäre Wahrscheinlichkeit, einen bestimmten Stpfl. in $m$ wiederzufinden. Der folgende Vergleich der statischen und der dynamischen Kontrolle beschränkt sich Landsberger/Meilijson(1982), Greenberg (1984) und Harrington (1988) folgend auf den Gleichgewichtszustand des Prozesses.

Aus (6.11) ergibt sich $g_{e}=q$ und $g_{m}=p$. Da nur Steuersünder in $e$ rückwirkend geprüft werden - in $m$ fand in der Vorperiode schon eine Prüfung statt -, lautet die Budgetgleichung der Finanzbehörde

$$
K=k p\left(1+g_{e} \theta\right)=k p(1+q \theta)
$$

Der Steuerausfall pro Kopf und pro Periode, den die Steuerhinterziehung verursacht, ist gleich

$$
g_{m}(1-p \pi)+g_{e}\left(1-p \pi\left(1+\frac{1}{\eta}\right)\right)=1-p \pi\left(1+\frac{q}{\eta}\right)
$$


Unter welchem Sytem ist bei gleichen Kosten der Fahndung der Steuerausfall am geringsten? Gleiche Kosten entstehen mit $p_{s}=p(1+q \theta)$ : Die Entdeckungswahrscheinlichkeit bei dem dynamischen System ist kleiner als $p_{s}$, da die rückwirkende Kontrolle finanziert werden muß. Setzt man $p_{s}$ in die Ungleichung

$$
1-p_{s} \pi>1-p \pi\left(1+\frac{q}{\eta}\right)
$$

ein und vereinfacht, ergibt sich $\theta<1 / \eta$ als Bedingung für die Überlegenheit dynamischer Kontrollen. Es sei an die Annahme $\eta<1$ erinnert. Die gefundene Bedingung ist also nicht sehr restriktiv: Solange die rückwirkende Kontrolle nicht mehr kostet als die Überprüfung der aktuellen Steuererklärung, lohnt sich ihre Durchführung.

Wir sind von der Voraussetzung ausgegangen, daß die Stpfl. notorisch hinterziehen. Wenn das Budget $K$ jedoch nicht ausreicht, um eine Entdeckungswahrscheinlichkeit $p>p_{C}$ zu finanzieren (siehe Abbildung 6.3), werden die Stpfl. nach einem Erfolg einmal ehrlich, um die Vergangenheit vergessen zu machen. Für diesen Fall ist daher die gerade vorgeführte Rechnung zu wiederholen.

Die stationäre Besetzung der Zustände $s, m$ und $e$ erhält man aus dem Gleichungssystem:

$$
\begin{aligned}
s: & p g_{s}+q g_{s}=g_{e} \\
m: & q g_{m}=p g_{s} \\
e: & g_{e}=q\left(g_{s}+g_{m}\right) \\
& g_{s}+g_{m}+g_{e}=1
\end{aligned}
$$

Die linke Seite der drei ersten Gleichungen enthält die Abgänge aus den einzelnen Zuständen. Die rechte Seite enthält die Zugänge aus dẹn anderen Zuständen. Betrachten wir z.B. die erste Zeile: $p \%$ der Stpfl. aus $s$ gelangt nach $m, q \%$ nach $e$; nach $s$ wechseln alle Mitglieder des Zustands $e$. Die letzte Gleichung des Systems fordert, daß sich die prozentualen Anteile zu eins addieren. Die Lösung des Systems ist schnell gefunden: Aus den letzten beiden Gleichungen erhält man $g_{e}=q /(1+q)$. Aus der ersten Gleichung des System ergibt sich $g_{s}=g_{e}$. Aus der zweiten folgt schließlich $g_{m}=p /(1+q)$.

In $m$ wird kein Stpfl. rückwirkend geprüft. Dies gilt auch für $e$, da dort alle ehrlich sind. Nur in $s$ wird bei einem Teil der Stpfl. rückwirkend kontrolliert: $p$ $\%$ in $s$ werden "erwischt"; $q g_{s}=q g_{e} \%$ von ihnen sind in der Vorperiode nicht geprüft worden und unterliegen daher jetzt einer rückwirkenden Kontrolle - die leider feststellt, daß die betreffenden Stpfl. in der Vorperiode ehrlich waren. Die Budgetgleichung der Behörde lautet damit:

$$
K=k p\left(1+\theta q g_{s}\right)=k p\left(1+\theta \frac{q^{2}}{1+q}\right)
$$


Der durch die Steuerhinterziehung verursachte Verlust ist

$$
\left(g_{s}+g_{m}\right)(1-p \pi)=\frac{1-p \pi}{1+q}
$$

Die statische und die dynamische Kontrolle führen zu gleichen Kosten, wenn

$$
p_{s}=p\left(1+\theta \frac{q^{2}}{1+q}\right)
$$

gilt. Der Steuerausfall ist nun mit der dynamischen Kontrolle bei gleichen Kosten geringer, wenn folgende Bedingung erfüllt ist:

$$
\begin{array}{ll} 
& 1-p \pi\left(1+\theta \frac{q^{2}}{1+q}\right)>\frac{1-p \pi}{1+q} \\
\Leftrightarrow \quad & 1-p \pi(1+q \theta)>0
\end{array}
$$

Sei $p^{*}$ durch $1-\pi p^{*}\left(1+q^{*} \theta\right)=0$ definiert. Mit $p<p^{*}$ sind die rückwirkenden den statischen Kontrollen überlegen, wenn gleichzeitig periodisch hinterzogen wird. $p$ muß mit anderen Worten in dem Intervall $\left(p_{C}, p^{*}\right)$ liegen. Wenn $\theta$ nicht zu groß ist, ist dieses Intervall nicht leer. Mit $\theta \leqq 1$ gilt $p^{*} \geqq p_{B}>p_{C}$ (siehe Abbildung 6.3).

$\mathrm{Ob}$ die Finanzbehörde bei rückwirkenden Kontrollen eine Entdeckungswahrscheinlichkeit festsetzt, die die Stpfl. zu periodischen oder zu notorischen Hinterziehern macht - in jedem Fall lohnt sich die Einführung rückwirkender Kontrollen, wenn $p<p^{*}$ gilt und die zusätzlichen Kosten der Prüfung der vorletzten Steuererklärung kleiner sind als die Kosten, die die Prüfung allein der letzten Steuererklärung verursacht. Wenn es mit anderen Worten "abnehmende Grenzkosten" bei einer Ausweitung der Kontrollen auf die Vergangenheit des Stpfl. gibt, sollten sie von der Finanzbehörde genutzt werden. Überraschenderweise gibt es eine obere Grenze $p^{*}$ für den Bereich der Entdeckungswahrscheinlichkeiten, in dem sich die Einführung rückwirkender Kontrollen lohnt. Da in der Praxis eine "hohe" Kontrollwahrscheinlichkeit jedoch nicht zu erwarten ist, ist die obere Grenze nicht sehr bedeutsam.

Der letzte Teil dieses Abschnitts geht einer Hypothese von Allingham/Sandmo (1972, S. 334) nach: Eine plausible Daumenregel zur Rationierung der für kostenträchtige Prüfungen zur Verfügung stehenden Mittel sei, die rückwirkenden Kontrollen abzubrechen, sobald sie kein zusätzliches Steueraufkommen erbringen. Diese Regel ist einleuchtend, wenn der Stpfl. in der Vergangenheit schon geprüft worden ist oder wenn die Verjährungsfrist überschritten ist. Sollten rückwirkende Kontrollen jedoch auch dann abbrechen, wenn der Stpfl. in einer Periode sein tatsächliches Einkommen deklariert hat?

Zur Beantwortung dieser Frage soll folgender Fall betrachtet werden. Sei $p \in\left(p_{C}, p_{B}\right)$, die Verjährungsfrist ein Jahr, und es sei wie bisher angenommen, daß 
die rückwirkende Kontrolle abbricht, wenn nicht hinterzogen wird. Unter diesen Bedingungen wird der Stpfl. in $e$ ehrlich sein und im übrigen hinterziehen. Wenn die Spielregeln jetzt in der Weise geändert werden, daß die Deklaration des tatsächlichen Einkommens die rückwirkende Kontrolle nicht länger aufhält, wird der Stpfl. zu einem notorischen Hinterzieher, da sich die Steuerehrlichkeit nicht mehr lohnt. Diese Verhaltensänderung erhöht die Kontrollkosten der Finanzbehörde um (siehe (6.12) und (6.14))

$$
k p(1+q \theta)-k p\left(1+\theta \frac{q^{2}}{1+q}\right)=k \theta p \frac{q}{1+q}>0
$$

da nun häufiger rückwirkende Kontrollen stattfinden. Andererseits sinkt der durch die Hinterziehung verursachte Steuerausfall um (siehe (6.13) und (6.15))

$$
1-p \pi\left(1+\frac{q}{\eta}\right)-\frac{1-p \pi}{1+q}=\frac{q}{1+q}\left(1-p \pi\left(1+\frac{q}{\eta}+\frac{1}{\eta}\right)\right)<0
$$

Das Vorzeichen folgt aus $\eta<1$ und der Annahme $p>p_{C} \Leftrightarrow 1-p \pi(1+q+1 / \eta)<0$ (siehe Abschnitt 6.2.2). Kontrollkosten und Steueraufkommen steigen infolge der Regeländerung. Der Nettoeffekt beider Änderungen kann im allgemeinen nicht bestimmt werden. Wenn jedoch die zusätzlichen Kosten einer rückwirkenden Kontrolle $\theta k$ nicht zu klein sind, kann sich die Regel von Allingham/Sandmo, daß rückwirkende Kontrollen abzubrechen sind, wenn nicht hinterzogen wird, als richtig erweisen.

\subsection{Verhaltensabhängige Entdeckungswahrscheinlichkeiten}

In diesem Abschnitt wird das Gegenstück zu den rückwirkenden Kontrollen und Strafen dargestellt (Landsberger/Meilijson, 1982, Greenberg, 1984, Harrington, 1988). Wird ein Steuerpflichtiger heute der Steuerhinterziehung überführt, hat dies nicht die Überprüfung seiner Vergangenheit zur Folge, sondern nur Konsequenzen für die Zukunft: In der nächsten Periode muß er mit einer höheren Entdeckungswahrscheinlichkeit und eventuell mit einer höheren Strafe rechnen. Wird andererseits heute festgestellt, daß das tatsächliche Einkommen der gerade zu Ende gegangenen Periode auch angegeben worden ist, können die Entdeckungswahrscheinlichkeit und die Strafe in der nächsten Periode sinken. Die Steuerpflichtigen werden also je nach ihrem entdeckten aktuellen Verhalten in Gruppen Verdächtiger und weniger Verdächtiger eingeteilt.

Es wird zunächst ein Modell mit zwei Gruppen dargestellt und seine Implikationen für das Verhalten der Steuerpflichtigen untersucht. Abschnitt 6.3.2 diskutiert, was die Erweiterung des Modells um eine dritte Klasse bewirkt, in die 
die Steuerpflichtigen gelangen, die bei zwei Prüfungen hintereinander "erwischt" werden. In Abschnitt 6.3.3 geht es um die Wirkung der Gruppenbildung auf das Steueraufkommen.

\subsubsection{Ein Modell mit zwei Gruppen: Verdächtige und weniger verdächtige Steuerpflichtige}

Die folgende Darstellung stützt sich auf Harrington (1988, S. 32 - 39). Dort wird zwar statt eines Steuerhinterziehers eine Firma betrachtet, die entweder die Kosten $c$ einer Umweltschutzmaßnahme trägt oder die Umwelt verschmutzt und riskiert, mit einer Wahrscheinlichkeit $p$ entdeckt zu werden und eine Strafe $F$ zu zahlen. Harringtons Analyse läßt sich jedoch direkt auf die Steuerhinterziehung übertragen: $c$ bezeichnet dann die fälligen Steuern und $F$ die Strafe inklusive der Steuernachzahlung.

Die Annahmen 2-5, 7 und 9 aus Abschnitt 6.2.1 werden übernommen: Der Stpfl. ist risikoneutral, lebt unendlich lange und diskontiert zukünftige Einkommen mit dem Faktor $\eta<1$. In jeder Periode erhält er das gleiche Einkommen und muß 1 DM Steuern zahlen. Für die hinterzogene Steuer in Zustand $i$ (siehe unten) in Periode $t$ gilt die Beschränkung $0 \leqq S_{i t} \leqq 1$. Infolge der Risikoneutralität kommen jedoch nur $S_{i t}=1$ oder 0 in Frage. Die Sequenz der $S_{i t}, t=0,1,2, \ldots$, legt der Stpfl. so fest, daß der Barwert aller erwarteten Erträge der Steuerhinterziehung möglichst groß ist. Die übrigen Annahmen beschreiben das System der Steuersicherung:

- Die Finanzbehörden ordnen jeden Stpfl. einer von zwei Gruppen zu ( $G_{1}$ und $G_{2}$ ), die sich durch unterschiedliche Entdeckungswahrscheinlichkeiten $p_{i}$ und Strafen $\alpha_{i}$ unterscheiden. Es gilt $p_{2}\left(1+\alpha_{2}\right)>p_{1}\left(1+\alpha_{1}\right)$. Die erwartete Strafe (inklusive der Steuernachzahlung) ist in der Gruppe der Verdächtigen $G_{2}$ größer als in der Gruppe der weniger Verdächtigen $G_{1}$.

- Ein Steuerhinterzieher in $G_{1}$ wandert in Gruppe $G_{2}$, wenn er entdeckt wird. Ein ehrlicher Stpfl. in $G_{2}$ gelangt zurück nach $G_{1}$, wenn seine Ehrlichkeit "entdeckt" wird. Findet keine Prüfung statt, bleibt der Stpfl. in der jeweiligen Gruppe.

Eine Politik legt für jede Periode $t$ und jeden Zustand $i \in\left\{G_{1}, G_{2}\right\}$ fest, ob 1 DM Steuern oder keine Steuer hinterzogen wird. Die optimale Politik ist stationär, da das in Abschnitt 6.2.2 angegebene Theorem auch hier anwendbar ist: $S_{i t}=S_{i}$ für alle $t$. Das Verhalten des Stpfl. hängt nur von dem Zustand $i \mathrm{ab}$, in dem er sich befindet, nicht aber von dem Zeitpunkt, zu dem er sich in diesem Zustand befindet. Da es nur die zwei Zustände $G_{1}$ und $G_{2}$ gibt, gibt es vier stationäre 
Politiken. Sei $\left(S_{i}\right)$ ein Vektor, dessen erste (zweite) Stelle die hinterzogene Steuer in $G_{1}\left(G_{2}\right)$ angibt. Die vier in Frage kommenden stationären Politiken sind $\left(S_{i}\right)=$ (00), (10), (01) und (11). Es wird für sie im folgenden die Notation $S^{00}, S^{10}, S^{01}$ und $S^{11}$ verwendet.

Mit der Annahme $1-p_{1}\left(1+\alpha_{1}\right)>0$ ist $S^{10}$ der Politik $S^{00}$ eines stets ehrlichen Stpfl. vorzuziehen. Zweitens ist $S^{11}$ unter dieser Bedingung $S^{01}$ überlegen (vgl. Harrington, 1988, S. 37). Wir haben es also entweder mit einem notorischen Steuerhinterzieher oder einem Stpfl. zu tun, der in Gruppe 1 hinterzieht und in Gruppe 2 freiwillig Steuern zahlt - in der Hoffnung entdeckt und nach $G_{1}$ transferiert zu werden.

Berechnen wir den diskontierten Ertrag der Steuerhinterziehung, den diese zwei Politiken liefern. Die Matrix der Übergangswahrscheinlichkeiten und die Matrix der laufenden Erträge der Steuerhinterziehung lauten für den periodischen Hinterzieher

$$
\begin{aligned}
& G_{1} \quad G_{2} \\
& \left(p_{i j}\left[S^{10}\right]\right)=\begin{array}{l}
G_{1} \\
G_{2}
\end{array}\left(\begin{array}{ll}
q_{1} & p_{1} \\
p_{2} & q_{2}
\end{array}\right) \\
& \begin{array}{ll}
G_{1} & G_{2}
\end{array} \\
& \left(r_{i j}\left[S^{10}\right]\right)=\begin{array}{l}
G_{1} \\
G_{2}
\end{array}\left(\begin{array}{cc}
1 & -\alpha_{1} \\
0 & 0
\end{array}\right)
\end{aligned}
$$

wobei $q_{i}=\left(1-p_{i}\right)$ ist. Die Kapitalwerte $E^{10}\left(G_{i}\right)$ der Politik $S^{10}$ sind die Lösungen des folgenden Gleichungssystems:

$$
\begin{aligned}
& E^{10}\left(G_{1}\right)=q_{1}-p_{1} \alpha_{1}+\eta q_{1} E^{10}\left(G_{1}\right)+\eta p_{1} E^{10}\left(G_{2}\right) \\
& E^{10}\left(G_{2}\right)=\eta\left(q_{2} E^{10}\left(G_{2}\right)+p_{2} E^{10}\left(G_{1}\right)\right)
\end{aligned}
$$

Löst man nach $E^{10}\left(G_{2}\right)$ auf, erhält man

$$
E^{10}\left(G_{2}\right)=\frac{\eta p_{2}\left(q_{1}-p_{1} \alpha_{1}\right)}{(1-\eta)^{2}+(1-\eta) \eta\left(p_{1}+p_{2}\right)}
$$

Die Übergangswahrscheinlichkeiten und Erträge des notorischen Hinterziehers sind

$$
\begin{aligned}
& G_{1} \quad G_{2} \\
& \left(p_{i j}\left[S^{11}\right]\right)=\begin{array}{l}
G_{1} \\
G_{2}
\end{array}\left(\begin{array}{cc}
q_{1} & p_{1} \\
0 & 1
\end{array}\right) \\
& G_{1} \quad G_{2} \\
& \left(r_{i j}\left[S^{11}\right]\right)=G_{1}\left(\begin{array}{cc}
1 & -\alpha_{1} \\
G_{2} & -\left(q_{2}-p_{2} \alpha_{2}\right)
\end{array}\right)
\end{aligned}
$$


Wenn er einmal nach $G_{2}$ gelangt ist, kommt er nicht wieder zurück nach $G_{1}$, da er nie steuerehrlich ist. Wenn der notorische Hinterzieher sich in $G_{2}$ befindet, kann sein diskontierter Ertrag unmittelbar angegeben werden:

$$
E^{11}\left(G_{2}\right)=\frac{q_{2}-p_{2} \alpha_{2}}{1-\eta}
$$

Ob die Politik $S^{10}$ oder $S^{11}$ vorteilhafter ist, hängt nicht von dem Zustand am Start des Prozesses ab (siehe das in Abschnitt 6.2.2 angegebene Theorem). Man kann daher $E^{10}\left(G_{2}\right)$ und $E^{11}\left(G_{2}\right)$ vergleichen, um die optimale Politik herauszufinden. Das Ergebnis des Vergleichs ist (vgl. Harrington, 1988, S. 37, Lemma 4)

$$
\begin{gathered}
E^{10}\left(G_{2}\right) \gtreqless E^{11}\left(G_{2}\right) \\
\Leftrightarrow \quad \frac{\eta p_{2}\left(p_{2}\left(1+\alpha_{2}\right)-p_{1}\left(1+\alpha_{1}\right)\right)}{1-\left(1-p_{1}\right) \eta} \gtreqless 1-p_{2}\left(1+\alpha_{2}\right)
\end{gathered}
$$

Aus dieser Äquivalenz folgt:

(i) Mit $1-p_{2}\left(1+\alpha_{2}\right) \leqq 0$ ist $S^{\mathbf{1 0}}$ die optimale Politik, da die Differenz der laufenden erwarteten Strafen der Hinterziehung in den zwei Gruppen, die der Zähler der linken Seite von (6.16) enthält, positiv ist.

Die Erklärung ist einfach: Die Steuerhinterziehung in $G_{2}$ verursacht nur Kosten man bleibt in $G_{2}$ und erzielt keinen positiven Ertrag.

(ii) Mit $1-p_{2}\left(1+\alpha_{2}\right)>0$ kann es optimal sein, notorisch zu hinterziehen, muß es aber nicht.

Selbst wenn $q_{2}-p_{2} \alpha_{2}>0$ gilt, kann es - anders als in einem Modell mit über die Zeit konstanten Entdeckungswahrscheinlichkeiten und Strafen - vorteilhaft sein, auf diesen Ertrag zu verzichten, um sich den Behörden bei einer Prüfung als ehrlicher Steuerzahler zu präsentieren und wieder in die Gruppe der weniger Verdächtigen $G_{1}$ eingeordnet zu werden - dort lohnt sich die Steuerhinterziehung dann nämlich um so mehr. "The irony of this model is that ... (taxpayers (R.H.)) with the status of 'good guys' are the ones, that can' afford to cheat, while those with a 'bad guy' reputation comply until moved back into $G_{1}$ " (Harrington, 1988, S. 38 - 39). Wenn die Differenz der laufenden Erträge der Steuerhinterziehung, $1-p_{1}\left(1+\alpha_{1}\right)-\left(1-p_{2}\left(1+\alpha_{1}\right)\right)$, jedoch zu klein ist, lohnt sich eine Rückkehr in $G_{1}$ eventuell nicht.

Unten wird folgende Tatsache eine Rolle spielen: Sinkt $\eta$, wird die linke Seite von (6.16) kleiner und die Rückkehr von $G_{2}$ nach $G_{1}$ weniger attraktiv. 


\subsubsection{Drei Gruppen}

In Greenberg (1984) wird das Modell aus Abschnitt 6.3.1 um eine dritte Gruppe $G_{3}$ erweitert, in die ein Stpfl. gelangt, wenn bei zwei Prüfungen hintereinander festgestellt wird, daß er Steuern verkürzt hat. $G_{3}$ weist zwei Besonderheiten auf:

- Der erwartete Ertrag der Steuerhinterziehung in $G_{3}, 1-p_{3}\left(1+\alpha_{3}\right)$, ist kleinergleich null.

- Aus $G_{3}$ geht kein Weg zurück nach $G_{2}$ und $G_{1}$. Gruppe 3 ist ein absorbierender Zustand.

Die laufenden Erträge der Hinterziehung in $G_{1}$ und $G_{2}$ sind positiv. Es wird jedoch keine Annahme darüber gemacht, welcher Ertrag größer ist ${ }^{6}$.

Eine Konsequenz von Greenbergs Modell kann direkt angegeben werden: Wer einmal in Gruppe 3 gelandet ist, wird nie wieder Steuern hinterziehen, da es sich nicht mehr lohnt. Es bleibt die Frage, wie sich der Stpfl. in den Gruppen $G_{1}$ und $G_{2}$ verhält, wenn ihm in $G_{2}$ ein Übergang nach $G_{3}$ droht. Diese Frage soll im folgenden behandelt werden. Im nächsten Abschnitt geht es dann darum, welchen Vorteil die Einrichtung einer dritten, absorbierenden Gruppe von "äußerst verdächtigen" Stpfl. für die Finanzbehörden haben kann.

In $G_{3}$ wird der Stpfl. immer ehrlich sein. In $G_{1}$ wird er stets hinterziehen, wenn $1-p_{1}\left(1+\alpha_{1}\right)>0$ gilt. Es bleiben die zwei Politiken $S^{100}$ und $S^{110}$. Es stellen sich die gewohnten Fragen: Welchen Ertrag liefern diese zwei Politiken? Welche Politik liefert den höchsten Ertrag? Sollte der Stpfl. also in $G_{2}$ ehrlich werden oder wie in $G_{1}$ sein gesamtes Einkommen hinterziehen?

Die Entscheidung für $S^{100}$ oder $S^{110}$ ist unabhängig davon, ob der Stpfl. in $G_{1}$ oder $G_{2}$ startet. Der Einfachheit halber lasse ich den Stpfl. wieder in $G_{2}$ starten. Den Ertrag von $S^{100}$ kennen wir bereits, da $E^{100}\left(G_{2}\right)=E^{10}\left(G_{2}\right)$ gilt. Mit der Politik $S^{100}$ wandert der Stpfl. - vorausgesetzt, er startet nicht in $G_{3}$ - zwischen $G_{1}$ und $G_{2}$ hin und her. Die dritte Gruppe erreicht er nie. Anders ist dies mit der Politik $S^{110}$. Der Stpfl., der sie verfolgt, wird früher oder später in $G_{3}$ landen. Der Ertrag $S^{110}\left(G_{2}\right)$ kann aus einer einzigen Gleichung berechnet werden.

$$
E^{110}\left(G_{2}\right)=q_{2}\left(1+\eta E^{110}\left(G_{2}\right)\right)-p_{2} \alpha_{2}
$$

Wenn der Stpfl. in $G_{2}$ nicht geprüft wird, spart er 1 DM Steuern, bleibt in $G_{2}$ und kann seine zukünftigen Erträge mit $\eta E^{110}\left(G_{2}\right)$ zusammenfassen. Wird er kontrolliert, zahlt er eine Strafe und gelangt in Gruppe 3, in der er zwangsläufig

\footnotetext{
${ }^{6}$ Durch die Einrichtung einer dritten Gruppe wird folgende Empfehlung umgesetzt: "Wer zweimal (hintereinander) lügt, dem glaubt man nicht, und wenn er auch die Wahrheit spricht".
} 
zu einem ehrlichen Steuerzahler wird. Die Auflösung der Gleichung nach $E^{110}\left(G_{2}\right)$ ergibt

$$
E^{110}\left(G_{2}\right)=\frac{q_{2}-p_{2} \alpha_{2}}{1-\eta q_{2}}
$$

Vergleichen wir diesen Kapitalwert mit $E^{11}\left(G_{2}\right)$ aus Abschnitt 6.3.1, stellt sich $E^{110}\left(G_{2}\right)<E^{11}\left(G_{2}\right)$ heraus. Da der Ertrag des Stpfl, der in $G_{2}$ ehrlich wird, nicht von der Existenz einer dritten Gruppe beeinflußt wird, kommen wir schon zu folgender Feststellung: Die Drohung der Finanzbehörde mit der absorbierenden Gruppe $G_{3}$ ist wirksam. Selbst wenn der Stpfl. in dem Modell mit zwei Gruppen in $G_{2}$ hinterzieht, kann er - mehr können wir bis jetzt nicht sagen - durch die dritte, absorbierende Gruppe dazu gebracht werden, in $G_{2}$ sein tatsächliches Einkommen zu deklarieren. In der Tat ist es ja eine harte Strafe, auf ewig zur Steuerehrlichkeit verurteilt zu sein. Betrachten wir jedoch im folgenden etwas genauer, wie wirksam die Drohung mit dieser Strafe ist.

Es gilt (vgl. Greenberg, 1984, S. 8):

$$
\begin{gathered}
E^{100}\left(G_{i}\right) \gtreqless E^{110}\left(G_{i}\right) \\
\Leftrightarrow \quad \frac{\eta p_{2}\left(1-p_{1}\left(1+\alpha_{1}\right)\right)}{(1-\eta)^{2}+(1-\eta) \eta\left(p_{1}+p_{2}\right)} \gtreqless \frac{1-p_{2}\left(1+\alpha_{2}\right)}{1-\eta q_{2}}
\end{gathered}
$$

Ist der laufende Ertrag der Hinterziehung in $G_{1}$ und $G_{2}$ positiv, kann nicht direkt gesagt werden, welche Seite dieser Ungleichung größer ist. Wenn man aber mit Greenberg (1984, S. 11, siehe auch S. 9) annimmt, daß "individuals ... do not discount the future at all ${ }^{\eta}$, ist $S^{\mathbf{1 0 0}}$ die optimale Politik. Mit $\eta \rightarrow 1$ strebt die linke Seite von (6.17) gegen unendlich, die rechte Seite gegen einen endlichen Wert. Mit einer positiven Wahrscheinlichkeit, in $G_{3}$ zu landen, ist der Ertrag eines notorischen Hinterziehers natürlich begrenzt. Die Politik $S^{100}$ liefert dagegen einen sehr großen Ertrag, wenn $\eta$ gegen eins strebt. Der Stpfl. gelangt aus $G_{2}$ immer wieder in die Gruppe $G_{1}$ zurück, in der er Steuern sparen kann, ohne Gefahr zu laufen, es für immer mit den Finanzbehörden zu verderben.

Wenn $\eta$ nicht sehr nahe bei eins liegt, ist nicht gesagt, daB $S^{110}$ die optimale Politik ist. Leider wird Ungleichung (6.17) ohne weitere Annahmen nicht informativer. Daher soll beispielshalber $p_{1}=p_{2}$ und $\alpha_{1}=\alpha_{2}$ angenommen werden. Sie lautet dann (siehe Anhang 6.3.2):

$$
p_{1}=p_{2} \gtreqless \frac{(1-\eta)(1+\sqrt{5})}{\eta 2} \approx \frac{1-\eta}{\eta} 1,6
$$

$(1-\eta) / \eta$ ist der durch $\eta$ implizit definierte Zinssatz. Wenn also die Wahrscheinlichkeit, von $G_{2}$ nach $G_{3}$ bzw. $G_{1}$ zu gelangen, klein ist relativ zu dem Zinssatz, mit dem der Stpfl. die Zukunft diskontiert, lohnt es sich nicht, in $G_{2}$ ehrlich zu 
werden und lange auf die Rückkehr nach $G_{1}$ zu warten. Das Beispiel zeigt übrigens, daß die Differenz der (positiven) laufenden Erträge in $G_{1}$ und $G_{2}$ nicht wie in dem Modell mit nur zwei Gruppen positiv sein muß, damit der Stpf. nach $G_{1}$ zurückgelangen möchte. Das Modell von Greenberg ist also auch dann interessant, wenn man $1-p_{1}\left(1+\alpha_{1}\right) \leqq 1-p_{2}\left(1+\alpha_{2}\right)$ annimmt.

\subsubsection{Gruppenbildung und Steueraufkommen}

Lohnt es sich aus der Sicht von Finanzbehörden, die mit ihrem Budget ein möglichst großes Steueraufkommen erzielen wollen, die Entdeckungswahrscheinlichkeiten von dem Verhalten der Stpfl. abhängig zu machen? Landsberger/Meilijson (1982) und Greenberg (1984) haben diese Frage für das Modell mit zwei bzw. drei Gruppen untersucht, wobei sie speziell annehmen, daß die Straffunktion in allen Gruppen die gleiche ist. Ihre Antworten sollen im folgenden dargestellt werden. Dabei wird die Unterscheidung je nach zwei oder drei Gruppen zunächst keine Rolle spielen.

Wenn $p_{1}, p_{2}>0$ gilt, die (identischen) Stpfl. in den zwei Systemen mit einer Gruppenbildung in $G_{1}$ hinterziehen und in $G_{2}$ ehrlich sind, und die steuerpflichtige Bevölkerung konstant bleibt, ändern sich die Größen der zwei Gruppen nicht mehr, wenn seit dem Start des Prozesses eine genügend lange Zeit vergangen ist. Unabhängig von der Verteilung der Bevölkerung auf die zwei Gruppen bei der Einführung der verhaltensabhängigen Kontrollen sind langfristig $g$ Prozent der Bevölkerung in $G_{1}$ und $1-g$ Prozent in $G_{2}$, wobei $g$ sich aus der Gleichung

$$
g p_{1}=(1-g) p_{2}
$$

bestimmt. In jeder Periode gehen $p_{1}$ Prozent der Mitglieder von $G_{1}$ nach $G_{2}$, und $p_{2}$ Prozent aus $G_{2}$ gehen zurück nach $G_{1}$, so daß sich $\mathrm{Zu}$ - und Abgänge saldieren. Man kann diesen Zusammenhang auch anders ausdrücken: Die Wahrscheinlichkeit, einen bestimmten Stpfl. in $G_{1}$ anzutreffen, nachdem seit dem Start der Prozesses $t$ Perioden vergangen sind, konvergiert mit $t \rightarrow \infty$ gegen $g=p_{2} /\left(p_{1}+p_{2}\right)$. Entsprechend ist $1-g=p_{1} /\left(p_{1}+p_{2}\right)$ die stationäre Wahrscheinlichkeit, einen bestimmten Stpfl. in $G_{2}$ wiederzufinden (vgl. Landsberger/Meilijson, 1982, S. 339). Wenn man wie Greenberg $(1984$, S. 6) gleich bei der Einführung verhaltensabhängiger Wahrscheinlichkeiten $1-g$ Prozent der Stpfl. der Gruppe $G_{2}$ zuordnet - und den Betreffenden per Bescheid mitteilt, daß sie bis zum "Beweis" des Gegenteils als sehr verdächtigte Steuerzahler eingestuft werden - , wird der Gleichgewichtszustand sogar sofort erreicht, wie Gleichung (6.18) zeigt ${ }^{7}$. Der folgende Vergleich der

\footnotetext{
${ }^{7}$ Beträgt die Wahrscheinlichkeit, einen bestimmten Stpfl. am Start des Prozesses in $G_{1}$ anzutreffen, gerade $g$, spricht man in der Theorie stochastischer Prozesse von einer stationären Anfangsverteilung der Markov-Kette, die die Wanderung eines Stpfl. zwischen den Gruppen $G_{1}$ und $G_{2}$ beschreibt.
} 
dynamischen Systeme der Steuersicherung mit der statischen Kontrolle beschränkt sich auf den Gleichgewichtszustand.

Mit einem Budget $K$ pro Kopf der Bevölkerung können mit statischen Kontrollen $p=K / k$ Prozent der Bevölkerung geprüft werden, wenn $k$ die Kosten je Prüfung sind. Das Budget soll so klein sein, daß $p<1 /(1+\alpha)$ gilt. Andernfalls hätte die Behörde ihren Auftrag, die Steuerhinterziehung zu begrenzen, schon vollends erfüllt (vgl. Landsberger/Meilijson, 1982, S. 343). In den dynamischen Systemen mit verhaltensabhängigen Entdeckungswahrscheinlichkeiten lautet die Budgetbeschränkung der Behörde

$$
K=k\left(g p_{1}+(1-g) p_{2}\right)
$$

wenn alle Stpfl. die Politik $S^{10}$ bzw. $S^{100}$ wählen: $p_{1}$ Prozent der Mitglieder von $G_{1}$ und $p_{2}$ Prozent der Mitglieder von $G_{2}$ werden in jeder Periode geprüft. Werden die Stpfl. nicht, wie angenommen, in $G_{2}$ ehrlich, würde (6.19) sehr schnell verletzt. In dem Modell mit zwei Gruppen landen alle Stpfl. in $G_{2}$, und die Behörde könnte ihre Ankündigung, in $G_{2}$ mit einer höheren Wahrscheinlichkeit als in $G_{1}$ zu prüfen, nicht durchhalten (ebenda, S. 343). In dem Modell mit drei Gruppen würden sich alle Stpfl. schließlich in $G_{3}$ wiederfinden, und die Ankündigung, daß sich die Steuerhinterziehung in $G_{3}$ nicht lohnt $\left(p_{3} \geqq 1 /(1+\alpha)\right)$, könnte aufgrund der begrenzten Mittel der Behörde nicht wahrgemacht werden. Die Wahrscheinlichkeiten $p_{1}$ und $p_{2}$ müssen bei der dynamischen Kontrolle folglich so festgesetzt werden, daß kein Stpfl. in $G_{2}$ hinterzieht. Auf diesen Punkt komme ich unten zurück.

Die statische und die dynamische Kontrolle verursachen gleiche Kosten, wenn $p=g p_{1}+(1-g) p_{2}$ gilt. Substituiert man die stationäre Wahrscheinlichkeit $g$, ergibt sich (Landsberger/Meilijson, 1982, S. 343)

$$
\begin{aligned}
p & =\frac{2 p_{1} p_{2}}{p_{1}+p_{2}} \\
\Leftrightarrow \quad p_{1} & =\left(\frac{2}{p}-\frac{1}{p_{2}}\right)^{-1}
\end{aligned}
$$

Aus der letzten Fassung geht hervor, daß $p_{1}$ nur auf Kosten von $p_{2}$ erhöht werden kann.

Der Rückgang des Steueraufkommens pro Periode und pro Kopf der Bevölkerung, den die Steuerhinterziehung verursacht, ist mit der statischen Kontrolle gleich $1-p(1+\alpha)$ und mit der dynamischen Kontrolle gleich $g\left(1-p_{1}(1+\alpha)\right)$, vorausgesetzt, in $G_{2}$ sind alle Stpfl. ehrlich. Unter welchem System ist der Verlust bei gleichen Kosten der Steuererhebung am geringsten? Setzt man $g=p_{2} /\left(p_{1}+p_{2}\right)$ und (6.20) in die Ungleichung

$$
1-p(1+\alpha) \gtreqless g\left(1-p_{1}(1+\alpha)\right)
$$


ein, erhält man

$$
1-p(1+\alpha) \gtreqless \frac{p}{2 p_{1}}-\frac{p}{2}(1+\alpha)
$$

bzw. $p_{1} \gtreqless p /(2-p(1+\alpha))=\check{p}_{1}$ (vgl. Landsberger/Meilijson, 1982, S. 344). Die kritische Wahrscheinlichkeit $\check{p}_{1}$, bei der beide Systeme zu einem gleich hohen Steuerausfall führen, ist größer null und kleiner eins. Es gibt folglich Wahrscheinlichkeiten $p_{1}>\check{p}_{1}$, die es lohnend machen, die statischen durch dynamische Kontrollen zu ersetzen - wenn man davon ausgehen kann, daß kein Stpfl. in $G_{2}$ hinterzieht. Genau dies ist jedoch eine berechtigte Frage, wie in den zwei vorangehenden Abschnitten gezeigt worden ist. Wenn $p_{1}$ zu groß und das entsprechende $p_{2}$ aus $(6.20)$ zu klein ist, sind die Stpfl. in $G_{2}$ möglicherweise nicht ehrlich, und die dynamische Kontrolle verletzt die Budgetbeschränkung der Finanzbehörde.

Wir müssen nun zwischen den Modellen mit zwei und drei Gruppen unterscheiden. Für die formale Analyse des Modells mit zwei Gruppen sei auf Landsberger/Meilijson (1982, S. 343 - 346) verwiesen. Sie definieren eine kritische Wahrscheinlichkeit $\hat{p}_{1}^{L}(\eta)$, bei der der Stpfl. indifferent zwischen den Politiken $S^{\mathbf{1 0}}$ und $S^{11}$ ist und bei der gleichzeitig die langfristige Budgetbeschränkung (6.20) erfüllt ist. Mit $p_{1}<\hat{p}_{1}^{L}(\eta)$ wird $S^{10}$ gewählt. Wenn die Finanzbehörde eine Entdeckungswahrscheinlichkeit $p_{1}$ aus dem Intervall $\left(\check{p}_{1}, \hat{p}_{1}^{L}(\eta)\right)$ festlegt (und $p_{2}$ aus $(6.20)$ berechnet), lohnt die Einführung verhaltensabhängiger Kontrollen. "Optimal" ist eine Wahrscheinlichkeit $p_{1}$, die sehr nahe links von $\hat{p}_{1}^{L}(\eta)$ liegt, da der Steuerausfall $p /\left(2 p_{1}\right)-(p / 2)(1+\alpha)$ mit $p_{1}$ sinkt. Die entscheidende Frage ist, ob das Intervall $\left(\check{p}_{1}, \hat{p}_{1}^{L}(\eta)\right)$ nicht leer ist. Landsberger/Meilijson (1982, S. 345 f.) zeigen, daß dies für einen Diskontsatz $\eta$ nahe bei eins nicht der Fall ist. Wenn $\eta$ sich von eins entfernt, wird das Intervall jedoch immer kleiner, bis es schließlich leer wird.

In dem Modell mit drei Gruppen kann man ebenfalls eine kritische Grenze $\hat{p}_{1}^{G}(\eta)$ definieren, bei der der Stpfl. nicht weiß, ob er auch in $G_{2}$ hinterziehen soll. Da die Stpfl. in dem Modell mit drei Gruppen eher ehrlich werden als bei zwei Gruppen, gilt $\hat{p}_{1}^{G}(\eta)>\hat{p}_{1}^{L}(\eta)$. Weiter gilt: Das Intervall $\left(\check{p}_{1}^{G}, \hat{p}_{1}^{G}(\eta)\right)$. kann auch dann nicht leer sein, wenn $\check{p}_{1}^{L} \geqq \hat{p}_{1}^{L}$ ist. Mit $\eta \rightarrow 1$ ist die Wirksamkeit von Greenbergs System der Steuerprüfungen natürlich am größten: Es muß dann nur $1 /(1+\alpha)>p_{1}>0$ und $p_{2}>0$ garantiert werden, damit alle Stpfl. die Politik $S^{100}$ verfolgen. Wenn also $p_{1}$ möglichst groß und $p_{2}$ entsprechend sehr klein festgelegt werden, werden die Zahl der Mitglieder von $G_{1}$ und der Steuerausfall, den sie verursachen, sehr klein.

Greenberg (1984, S. 6 und 4) betont die Wirksamkeit seiner Methode, indem er nicht den Steuerausfall, sondern die Anzahl der Steuerhinterzieher betrachtet und sich auf den Fall $\eta \rightarrow 1$ konzentriert. Mit der statischen Kontrolle und $p<1 /(1+\alpha)$ hinterzieht jeder Steuern. In dem Modell mit drei Gruppen kann die Finanzbehörde dagegen den Anteil der Steuerhinterzieher in der Bevölkerung auf 
jeden beliebigen Prozentsatz $\bar{g}>0$ festlegen! Prüft sie in $G_{1}$ mit der Wahrscheinlichkeit $p / 2$ und in $G_{2}$ mit der Wahrscheinlichkeit $\bar{g} p /(1-\bar{g}) 2$, hinterziehen im Gleichgewicht $g=\bar{g}$ Prozent und die "tax authorities remain (well) within their budget constraint” (Greenberg, 1984, S. 9):

$$
\bar{g} \frac{p}{2}+(1-\bar{g}) \frac{\bar{g} p}{(1-\bar{g}) 2}=\bar{g} p \leqq p
$$

Ganz verhindert werden kann die Steuerhinterziehung natürlich nicht, da $1 /(1+$ $\alpha)>p_{1}>0$ und $p_{2}>0$ Voraussetzungen für die Optimalität der Politik $S^{100}$ sind. Die Gruppe $G_{1}$ wird immer einige Mitglieder aufweisen.

Sprechen wir einen wichtigen Punkt noch deutlicher aus: Die Behörde sollte ihr Budget gar nicht voll verausgaben, wenn sie die Zahl der Hinterzieher möglichst klein halten will! Löst $\operatorname{man} \bar{g}=p_{2} /\left(p_{1}+p_{2}\right)$ nach $p_{2}$ auf und setzt $p_{2}=\bar{g} p_{1} /(1-\bar{g})$ in Gleichung (6.19) ein, ergibt sich

$$
\bar{g}=\frac{K}{k 2 p_{1}}
$$

Kann das Ziel $\bar{g}$ durch eine weitere Erhöhung von $p_{1}$ nicht erreicht werden, da $p_{1}$ schon nahe bei $1 /(1+\alpha)$ liegt, gibt die Behörde einfach weniger Geld für die Steuerfahndung und mehr für andere Dinge aus ( $K$ sinkt) oder erhöht die Kosten einer Prüfung durch höhere Spesenabrechnungen der Fahnder ( $k$ steigt), um die Wahrscheinlichkeit einer Prüfung in Gruppe $G_{2}$ zu senken.

Die zwei Modelle mit drei und zwei Gruppen kommen zu der gleichen Empfehlung an die Behörden. Die Entdeckungswahrscheinlichkeit in $G_{1}$ sollte möglichst groß, und die Entdeckungswahrscheinlichkeit in $G_{2}$ möglichst klein festgelegt werden. Ein großer Teil (bei Greenberg der größte Teil) der Bevölkerung befindet sich in $G_{2}$ und muß dort "schmoren": Nach $G_{1}$ kommt ein Stpfl. nur nach langer Zeit zurück ( $p_{2}$ ist klein). Ist er dank seines Wohlverhaltens einmal nach $G_{1}$ gewechselt, lohnt sich die Steuerhinterziehung zwar. Aber die hinterzogenen Steuern muß er mit einer hohen Wahrscheinlichkeit nachzahlen, um anschließend wieder auf die "Durststrecke" der Gruppe 2 zu gelangen. Wird dagegen ein $p_{1}<\check{p}_{1}$ gewählt und ist $p_{2}$ entsprechend groß, führen die verhaltensabhängigen Kontrollen zu einem geringeren Steueraufkommen als die Prüfungen mit einer stets gleich hohen Wahrscheinlichkeit. Die Stpfl. werden in $G_{1}$ selten entdeckt. Wenn dies aber einmal der Fall ist, kommen sie schnell wieder aus $G_{2}$ zurück, weil die durchschnittliche Verweildauer dort kurz ist.

Die Empfehlung, $p_{1}$ auf Kosten von $p_{2}$ zu erhöhen, kann schnell auf eine Grenze treffen, wenn zukünftige Einkommen für die Stpfl. ein geringeres Gewicht als heutige Einkommen haben und sie daher auf die Rückkehr von $G_{2}$ nach $G_{1}$ nicht warten wollen. In dem Modell von Landsberger/Meilijson ist diese Grenze verständlicherweise schneller erreicht als in dem Modell mit drei Gruppen. Aber auch hier 
gibt es sie, so daß die Entdeckungswahrscheinlichkeit $p_{2}$ nicht zu klein gewählt werden kann und die Zahl der Steuerhinterzieher folglich nicht beliebig klein wird. Greenberg (1984, S. 9) schlägt daher vor, den Schrecken der absorbierenden, dritten Gruppe zu erhöhen, indem die gesamte steuerliche Vergangenheit der Stpfl., die $G_{3}$ betreten, aufgerollt wird und rückwirkende Strafen verhängt werden. Die abschreckende Wirkung dieser Maßnahme ist jedoch, wie in Teil 2 dieses Kapitels gezeigt worden ist, begrenzt, wenn die Steuerhinterziehung nach wenigen Jahren verjährt ist. Zudem gibt es einen guten Grund dafür, die Drohung mit der dritten Gruppe nicht zu übertreiben. Die Bürger könnten sie wegen des begrenzten Budgets der Finanzbehörde in eine leere Drohung verwandeln, wenn sie sich einig würden, immer zu hinterziehen. Dann wären nach einiger Zeit alle in $G_{3}$, könnten aber nur mit $p=K / k$ geprüft werden. Greenberg $(1984$, S. 10) bezeichnet es zwar als "unrealistic to assume that individuals form coalitions and decide to 'beat the system' with correlated strategies". Ob dies jedoch auch für sein Heimatland gilt, ist fraglich. Denn die Steuermoral in Israel ist vielleicht ein Grund dafür, daß dort einige der interessantesten Aufsätze über die Steuerhinterziehung geschrieben worden sind.

\subsection{Zusammenfassung und Diskussion}

In dynamischen Modellen der Steuerhinterziehung sind die Strafe und die Entdeckungswahrscheinlichkeit von dem Verhalten des Steuerpflichtigen in der Vergangenheit abhängig. Bei rückwirkenden Kontrollen erhöht die zuvor nicht entdeckte Steuerhinterziehung die Strafe. Bei zeitlich variablen Entdeckungswahrscheinlichkeiten erhöht die entdeckte Hinterziehung die zukünftige Wahrscheinlichkeit einer Steuerprüfung. Wohlverhalten des Steuerpflichtigen - die Deklaration des tatsächlichen Einkommens - führt dagegen dazu, daß Strafe und Entdeckungswahrscheinlichkeit sinken. "In effect, these models work by offering a bribe and threatening a penalty at the same time" (Harrington, 1988, S. 48). Die Reaktion eines Steuerpflichtigen auf dieses Angebot ist nur allzu verständlich. Er wird nach einer bestimmten Anzahl von Jahren, in denen er erfolgreich hinterzogen hat, einmal ehrlich, um eine hohe rückwirkende Strafe zu vermeiden oder in eine Gruppe von weniger verdächtigen Steuerzahlern zu gelangen, wenn die Strafsätze und die Entdeckungswahrscheinlichkeiten nicht so niedrig sind, daß er vorzieht, ein notorischer Steuerhinterzieher zu bleiben. Ist der Stpfl. einmal ehrlich, verzichtet er zwar auf eine mögliche Steuerersparnis in dem betreffenden Jahr, schafft sich dafür aber für die nächsten Jahre eine bessere Ausgangsposition. Diese Politik kann auch dann von Vorteil sein, wenn der laufende Ertrag der Steuerhinterziehung positiv ist. Damit sind die dynamischen Modelle in der Lage, die Steuerpflichtigen in bestimmten 
Jahren (genauer Zuständen) trotz eines unmittelbaren Anreizes zur Hinterziehung zu ehrlichen Steuerzahlern zu machen.

Die "Bestechung" der Steuerpflichtigen hat für die staatlichen Stellen jedoch auch eine Kehrseite. Wenn rückwirkende Kontrollen abgebrochen werden, sobald der Steuerpflichtige in einer Periode sein tatsächliches Einkommen angegeben hat, kann eine Verlängerung der Verjährungsfrist für die Steuerhinterziehung zu einer stumpfen Waffe werden. Der Hinterzieher sorgt dann selbst für die "Verjährung" seiner Taten, bevor ihn die maximale Strafe, die mit der Verjährungsfrist steigt, treffen kann.

Die zwei in diesem Kapitel dargestellten Modelle weisen nicht nur in bezug auf die positive Analyse, sondern auch in normativer Hinsicht ähnliche Eigenschaften auf. Bei gleichen Kosten der Kontrollen führen sie zu einem höheren Steueraufkommen als das statische Modell. Man kann diesen Zusammenhang auch anders ausdrücken: Bei gleichem Aufkommen (bzw. gleichem Aufkommensverlust durch die Steuerhinterziehung) können dynamische Kontrollen mit geringeren Mitteln auskommen als statische Kontrollen, so daß der Staat weniger für die Steuerfahndung und mehr für andere Dinge ausgeben oder Steuern senken kann. Wie eine mögliche Ersparnis von Kontrollkosten verteilt wird, ist also eine zweite Frage ${ }^{8}$.

Auf den letzten Seiten dieses Kapitels sollen drei Annahmen der dargestellten dynamischen Modelle diskutiert werden. Es handelt sich dabei zum einen um die Risikoneutralität der Steuerpflichtigen und ihr in jeder Periode gleich hohes Einkommen. Die dritte Annahme, zu der einige Bemerkungen angebracht sind, lautete: Der Strafsatz, mit dem die hinterzogene Steuer multipliziert wird, um die Geldstrafe zu berechnen, ist so klein, daß die Finanzbehörde mit statischen Kontrollen in keiner Periode ehrliche Steuerzahler erreichen kann.

Risikoneutralität ist sicher eine sehr spezielle Annahme. Es ist daher wünschenswert, die dynamischen Modelle für risikoaverse Steuerpflichtige zu verallgemeinern. Dies ist in der Literatur zum Teil schon geschehen. Landsberger/Meilijson (1982, S. 346 - 349) zeigen, daß in dem Modell mit zwei Gruppen verdächtiger und weniger verdächtiger Steuerpflichtiger bei Risikoaversion ebenfalls ein Anreiz besteht, in der zuerst genannten Gruppe $\left(G_{2}\right)$ ehrlich zu werden. Weiter zeigen sie, daß (zumindest) für ein "kleines" Budget der Behörde (eine kleine Entdeckungswahrscheinlichkeit bei statischen Kontrollen) die dynamische Kontrolle bei gleichen Kosten ein höheres Aufkommen liefern kann. Daß statische Kontrollen bei Risikoaversion auch für ein "mittelgroßes" Budget schlechter abschneiden als das von

\footnotetext{
${ }^{8}$ Die Entdeckungswahrscheinlichkeit kann statt von der Vergangenheit des Steuerpflichtigen von dem deklarierten Einkommen der neuesten Steuererklärung abhängig gemacht werden (siehe Abschnitt 2.2). Reinganum/Wilde (1986) zeigen, daB sich dies für ein nettoeinnahmenmaximierendes Finanzamt lohnen kann, wenn es die Verteilung des Einkommens kennt, das der Steuerpflichtige verdient.
} 
Landsberger/Meilijson vorgeschlagene System der Steuersicherung, ist zwar anzunehmen, bisher aber nicht untersucht worden. Für das Modell mit drei Gruppen kann Greenberg (1984) entnommen werden, daß sich mit einer Nutzenfunktion des Steuerpflichtigen

$$
\sum_{t=0}^{\infty} \eta^{t} U\left(C_{t}\right)
$$

(mit $C_{t}$ als Einkommen nach Steuer und Strafe in Periode $t$ ) im Fall $\eta \rightarrow 1$ für $U^{\prime \prime}\left(C_{t}\right)<0$ entsprechende Ergebnisse wie bei einem risikoneutralen Steuerpflichtigen ergeben. Bei rückwirkenden Kontrollen haben Allingham/Sandmo (1972) folgendes Resultat erhalten: Innerhalb einer Serie von Jahren, in denen die Steuerhinterziehung nicht entdeckt wird, deklariert ein risikoaverser Steuerzahler von Periode zu Periode ein höheres Einkommen, da die zu erwartende Strafe steigt und dies einen risikoaversen im Gegensatz zu einem risikoneutralen Steuerpflichtigeren davon abhält, stets das gesamte Einkommen zu hinterziehen. Wird der Steuerpflichtige entdeckt oder hat er selbst seine Erfolgsserie abgebrochen, beginnt er im nächsten Jahr wieder mit einem kleinen deklarierten Einkommen.

Was ändert die Risikoaversion allgemein gesprochen in den dynamischen Modellen? Der Steuerpflichtige wird nicht unbedingt sein gesamtes Einkommen hinterziehen, wenn er überhaupt Steuern verkürzt. Eine innere Lösung kann vorzuziehen sein. In dem Modell mit rückwirkenden Kontrollen ist ein weiterer Effekt zu erwarten: Ich vermute, daß ein risikoaverser Steuerpflichtiger eine Erfogsserie eher abbricht als ein risikoneutraler Entscheidungsträger, da die rückwirkende Strafe für ihn schwerer wiegt. Der zentrale Punkt der dynamischen Modelle wird bei Risikoaversion erhalten, wenn nicht gar noch stärker betont werden: Die Steuerpflichtigen haben einen Anreiz, sich nicht in jeder Periode (jedem Zustand) gleich zu verhalten, sondern ihre Deklarationsentscheidungen in Abhängigkeit von ihrer Geschichte zu variieren.

Es ist in diesem Kapitel angenommen worden (mit der Ausnahme von Abschnitt 6.2.4), daß der Steuerpflichtige in jeder Periode ein gleich hohes Einkommen verdient. Die Modelle mit wiederholter Veranlagung sind mit dieser Annahme aber auf die Dauer nicht plausibel. Die Finanzbehörde wird nach einigen Prüfungen merken, daß der Steuerpflichtige ein festes Einkommen $Y$ hat, und sich die Frage stellen, ob sie ihn in Zukunft nicht automatisch prüfen sollte, wenn er nicht mindestens $Y$ angibt.

Diesem Einwand kann man begegnen, indem man von einem endogenen oder stochastischen Einkommen ausgeht, so daß es für die Behörde schwieriger wird, aus den vorliegenden Akten auf das zukünftige Einkommen des Steuerpflichtigen zu schließen. Die Berücksichtigung eines endogenen Einkommens in dynamischen Modellen der Steuerhinterziehung ist eine Aufgabe für die zukünftige Forschung. Ein stochastisches Einkommen ist zum Teil berücksichtigt worden. Greenberg (1984, S. 
3 und 4) ist dies besonders leicht gefallen: Das aktuelle Einkommen soll zwar eins aus einer Menge vieler möglicher Einkommen sein. Da die Steuerpflichtigen bei ihm aber des Glaubens sind, sie werden in Zukunft gerade das Einkommen erhalten, das sie in diesem Jahr verdient haben, unterscheidet sich Greenbergs Modell analytisch in nichts von einem Modell mit festem Einkommen. Überzeugender ist die Annahme, daß der Steuerpflichtige eine Vorstellung über die Verteilung seiner zukünftigen Einkommen hat. Landsberger/Meilijson (1982) gehen von unabhängig identisch und kontinuierlich verteilten Einkommen in den einzelnen Perioden aus. Das aktuelle Einkommen kennt der Steuerpflichtige natürlich schon. Es sei mit $Y_{i}$ bezeichnet, wenn der Steuerpflichtige sich in Gruppe $i$ befindet $(i=1,2)$. Landsberger/Meilijson zeigen, daß es für jede Gruppe ein kritisches Einkommen $Y_{i}^{*}$ gibt, bei dem der Steuerpflichtige nicht weiß, ob er in der betreffenden Gruppe ehrlich sein sollte oder nicht. Mit $Y_{1}<Y_{1}^{*}$ lohnt es sich nicht, in Gruppe $1 \mathrm{zu}$ hinterziehen. Die mögliche Steuerersparnis ist zu gering, um eine Versetzung nach Gruppe $2 \mathrm{zu}$ riskieren. Der Steuerpflichtige wartet lieber darauf, daß er in der nằchsten Periode ein höheres Einkommen hat. In Gruppe 2 verzichtet der Steuerpflichtige mit $Y_{2}>Y_{2}^{*}$ auf die Chance, in Gruppe 1 zurückzukehren. Der laufende Ertrag ist im Vergleich zu den in Zukunft zu erwartenden Erträgen dann so groß, daß auf eine Versetzung nach Gruppe 1 noch gewartet werden kann. Mit einem stochastischen Einkommen trifft der Steuerpflichtige in den zwei Gruppen nicht jeweils immer dieselbe Entscheidung. Er macht sie neben der Gruppenzugehörigkeit auch von dem aktuellen Einkommen abhängig. Diese Eigenschaft hatte auch das Modell mit rückwirkenden Kontrollen in Abschnitt 6.2.4, in dem das Einkommen des Steuerpflichtigen zweipunktverteilt war.

Kommen wir zu dem wirklich kritischen Punkt der dynamischen Modelle. Er betrifft ihre normative Bedeutung. Mit einem beschränkten Budget der Finanzbehörde und einem vorgegebenen, hinreichend kleinen Strafsatz können die dynamischen Kontrollen den durch die Steuerhinterziehung verursachten Rückgang des Steueraufkommens verringern. Die genannten Restriktionen mögen für viele Finanzbehörden durchaus realistisch sein. Wenn sie ihrem Auftrag gerecht werden wollen, die Steuerhinterziehung zu begrenzen, hat die Frage, welche Methode der Steuersicherung bei gleichen Kosten das höchste Aufkommen liefert, eine gewisse Bedeutung. Es fragt sich aber, warum die den Finanzbehörden übergeordneten staatlichen Instanzen diesen nicht die Arbeit erleichtern und für die Steuerhinterziehung einen so hohen Strafsatz festlegen, daß sich diese Aktivität auch bei verschwindend geringen (konstanten) Entdeckungswahrscheinlichkeiten nicht mehr lohnt. Mit einer sehr kleinen konstanten Entdeckungswahrscheinlichkeit würde der Einsatz dynamischer Kontrollen allerdings an Interesse verlieren: Wegen sehr kleiner Vorteile - ein etwas höheres Aufkommen oder etwas geringere Kosten - lohnt sich der Aufwand nicht. 
Auf die gestellte Frage findet man in der Literatur zur Steuerhinterziehung nur bei Harrington (1988, S. 31 f.) eine kurze Antwort, deren Kernsatz lautet: "Severe but rarely-imposed penalties might seem capricious and unfair". Damit stellt sich allerdings gleich die nächste Frage. Warum sollen drakonische Strafen (genauer Strafsätze) ungerecht sein? Zwei Gründe gibt Harrington selbst am Schluß seines Aufsatzes an - was nicht heißt, daß es nicht weitere gibt:

- Nicht alle Steuerpflichtigen verhalten sich stets "rational". Gesetze werden nicht nur absichtlich, sondern auch versehentlich verletzt - ohne daß die Gerichte jedoch wissen, aus welchem Grund ein Verstoß zustandegekommen ist (vgl. Rubinstein, 1979, S. 406 f.).

- Das im Rahmen einer steuerlichen Prüfung festgestellte Einkommen entspricht nicht unbedingt dem tatsächlichen Einkommen. Die Finanzbehörde stellt es nur mit einer Abweichung nach oben oder unten fest, so daß ein Hinterzieher trotz Prüfung ohne Strafe bleiben kann und ein ehrlicher Steuerzahler bei einer Abweichung nach oben belangt wird ${ }^{9}$.

Ein Versehen kann jedem unterlaufen, der nicht stets auf alles Acht geben kann oder möchte ${ }^{10}$. Es äußerst hart zu bestrafen, wird daher nicht auf verbreitete Zustimmung stoßen. Für Fehler der Finanzbehörden zu büßen, werden die Steuerzahler ebenfalls als ungerecht ansehen. Der Staat steht daher in einem statischen Modell vor einem Dilemma: Hohe Strafen können ungerecht sein, geringe Strafen aber führen zu einer verbreiteten Mißachtung der Gesetze (vgl. Rubinstein, 1979, S. 406 f.).

Die dynamischen Modelle der Steuerhinterziehung bieten einen Ausweg aus diesem Dilemma an. Sie ersetzen den hohen Strafsatz durch andere Formen der

\footnotetext{
${ }^{9}$ Diese Komplikation berücksichtigen Scotchmer/Slemrod (1989) in dem Grundmodell der Steuerhinterziehung. Sie ist relevant für die Selbstveranlagung, bei der der Steuerpflichtige selbst das zu versteuernde Einkommen (die Steuerbemessungsgrundlage) berechnet. Bei der Veranlagung durch die Finanzbehörde dagegen übermittelt der Steuerpflichtige dieser einen Teil der relevanten Tatbestände (Einkünfte, Abzüge, Familienstand, etc.). Die Behörde berechnet dann das zu versteuernde Einkommen. Bei der Abgabe seiner Steuererklärung kennt der Steuerpflichtige diese Größe nicht genau, wenn z.B. bei bestimmten Abzügen nicht vorhersagbar ist, ob sie anerkannt werden (das Streichen der Abzüge führt aber zu keiner Strafe). Das hinterzogene Einkommen kennt der Steuerpflichtige im Gegensatz zu dem zu versteuernden Einkommen jedoch, wenn er steuerpflichtige Einkünfte nicht deklariert. Zumindest bei proportionalem Tarif hängt die Strafe damit anders als bei Scotchmer/Slemrod (1989, S. 20) nicht davon ab, mit welcher Präzision (welchem Fehler nach oben und unten) die Behörde das zu versteuernde Einkommen feststellt. Der Ansatz von Scotchmer/Slemrod ist daher bei einer Veranlagung durch die Behörden weniger relevant.

${ }^{10}$ Die Allokation von Aufmerksamkeit kann überlegt sein. Insofern passieren auch rationalen Entscheidungsträgern Versehen.
} 
Strafe: Eine Kumulation der Strafen oder eine Prüfung mit einer höheren Wahrscheinlichkeit. Beide scheinen gesellschaftlich akzeptabel zu sein, zumal wenn der Steuerpflichtige wiederholt hinterzogen hat. Der Widerspruch ist nur, daß die Gründe für die Beschränkung der Strafsätze nicht berücksichtigt werden. Die Steuerpflichtigen sind auch in den dynamischen Modellen stets rational, die Behörde arbeitet fehlerlos. Ohne diese Voraussetzungen würden die dynamischen Kontrollen in ihrer in diesem Kapitel dargestellten Form ihren Vorteil gegenüber statischen Kontrollen verlieren. In dem Modell von Greenberg z.B. würden alle Steuerpflichtigen schließlich in der absorbierenden, dritten Gruppe landen - und die Drohung, daB sich die Hinterziehung dort nicht lohnt, könnte wegen der begrenzten Mittel der Steuerfahndung nicht wahrgemacht werden. Stellt das Finanzamt das tatsächliche Einkommen nur mit einem Fehler (nach oben oder unten) fest, lohnt es sich vielleicht nicht mehr, in bestimmten Zuständen ehrlich zu werden: Wenn man ehrlich ist, muß man eventuell doch eine Strafe zahlen, und wenn man hinterzieht, kommt man trotzdem davon.

Was immer die Gründe für mäßig hohe Strafsätze sein mögen - sie sollten in den dynamischen Modellen Berücksichtigung finden, wenn diese Empfehlungen für die Organisation der Steuerfahndung geben wollen. Die oben angegebenen Gründe könnten z.B. dazu führen, im Rahmen rückwirkender Kontrollen die Steuerhinterziehung in den ersten Jahren geringer als die Taten in späteren Perioden einer längeren entdeckten Erfolgsserie zu bestrafen, da eine Erfolgsserie um so weniger auf Versehen der Steuerzahler und Fehler der Behörden zurückzuführen sein wird, je länger sie ist. Die Anzahl der Gruppen, die bei variablen Entdeckungswahrscheinlichkeiten vor eine absorbierende Gruppe geschaltet werden, könnte erhöht werden, so daß es unwahrscheinlich ist, diese nur infolge von Fehlern und unbeabsichtigten Gesetzesverstößen zu erreichen. Rubinstein (1979, S. 407) folgend würde der Hinterzieher nur dann (hart) bestraft, "if his long-run record is 'unreasonably' bad". Konzessionen dieser Art würden die Überlegenheit dynamischer Kontrollen sicher vermindern. Ich vermute aber, daß es sich dennoch auszahlt, Strafen und Entdeckungswahrscheinlichkeiten von der Geschichte eines Steuerpflichtigen abhängig zu machen, wenn die Begrenzung der Steuerhinterziehung als ein wichtiges Ziel der Finanzpolitik anzusehen ist. 


\section{Zusammenfassung: Steuerhinterziehung und Finanzpolitik}

\section{Die Modelle}

Die vorliegende Arbeit hat Modelle eines Steuerpflichtigen untersucht, der versucht, einen Teil seiner Steuerschuld zu hinterziehen. Es handelte sich stets um einen sehr versierten Steuerhinterzieher: Über alle für ihn bedeutsamen Rahmenbedingungen ist er informiert. Er kennt nicht nur das Steuerrecht, sondern auch die Wahrscheinlichkeit einer steuerlichen Prüfung und die zu erwartenden Strafen für die Steuerhinterziehung; seine Entscheidungen über die Ersparnis oder das Arbeitsangebot macht er weitblickend davon abhängig, in wieweit sich die Hinterziehung seines Einkommens lohnt. Als weniger versiert wurden die Finanzbehörden dargestellt. In den statischen Modellen prüfen sie den Steuerpflichtigen mit einer gegebenen Wahrscheinlichkeit (wenn man die Entdeckungswahrscheinlichkeit als objektive Größe interpretiert) und machen die Geldstrafe nur von dem Steuervergehen in der laufenden Periode abhängig. Es wurden aber auch Modelle behandelt, in denen die Kontrollen und Strafen von dem Verhalten des Steuerhinterziehers in der Vergangenheit abhängig sind, so daß dieser ein dynamisches Optimierungsproblem lösen muß.

Die Arbeit hat gezeigt, daß sich der Bereich der positiven Theorie, der sich mit statischen Modellen befaßt, auf einen Zustand der Konsolidierung zubewegt. Die vorhandenen dynamischen Modelle dagegen "are a beginning, not the end" (Cowell, 1986, S. 197). Die effiziente Auswertung von Informationen über die Steuerpflichtigen, die insbesondere früheren Veranlagungen entnommen werden können, ist ein vielversprechendes Thema. Wenn Kontrollen und Strafen von dem Verhalten des potentiellen Hinterziehers abhängig gemacht werden, ist jedoch folgendes zu beachten: Die angekündigte Verhaltensweise der Finanzbehörde muß glaubwürdig sein. Ist sie das nicht - etwa weil die Behörde schon einmal ihre Strategie geändert hat -, ist die Unterstellung der statischen Modelle, der Hinterzieher 
kalkuliere mit einer gegebenen subjektiven Wahrscheinlichkeit, vielleicht eine gute Näherung.

\section{Strafen und Kontrollen oder Steuerpolitik?}

Die Ergebnisse der Arbeit werden im folgenden nicht kapitelweise, sondern im Querschnitt zusammengefaßt. Ich nehme dabei auf die drei Fragen bezug, die in der Einleitung gestellt worden sind (siehe a), b) und c) in der Aufgabenstellung). Die Quintessenz der dargestellten Modelle wird allgemein formuliert - auch wenn jedes (noch so allgemeine) Modell im Grunde eine Beispiel ist.

Steuern werden in einem statischen Modell genau dann hinterzogen, wenn der erwartete Ertrag der Hinterziehung des laufenden Einkommens positiv ist. In einem dynamischen Modell ist diese Bedingung nicht hinreichend: Ein Steuerhinterzieher wird in bestimmten Perioden ehrlich, wenn er sich dadurch in der Zukunft eine bessere Ausgangsposition verschaffen kann.

Mit wachsenden Einkommen der Steuerpflichtigen wird die Steuerehrlichkeit - gemessen an der hinterzogenen Steuer oder dem hinterzogenen Einkommen abnehmen. Diese Aussage bestätigt sich in vielen Modellvarianten, wenn man von einer abnehmenden absoluten Risikoaversion ausgehen kann. Gesichert ist dieser Zusammenhang jedoch keineswegs. Wenn die Geldstrafe progressiv in dem Sinne ist, daß Steuerpflichtige mit einem höheren Nettoeinkommen eine höhere Strafe pro DM hinterzogener Steuern zahlen, ist z.B. schon nicht gesagt, daß höhere Einkommen zu großen Teilen hinterzogen werden.

Welcher Einfluß geht von der Finanzpolitik auf die Steuerhinterziehung aus? Die Antwort auf diese zentrale Frage hat die Steuerpolitik auf der einen, Strafen und Kontrollen auf der anderen Seite zu unterscheiden. Für Strafen und Kontrollen gilt in vielen Modellvarianten: Sie sind geeignete Mittel, um den Steuerbetrug einzudämmen. Sowohl in den statischen als auch in den dynamischen Modellen haben sie die gewünschte Wirkung - zumindest solange man von einem exogenen Einkommen des Steuerpflichtigen ausgeht. Zusätzlich kann zum Teil gesagt werden, welche Form von Kontrollen und Strafen am wirksamsten ist. Es lohnt sich z.B., Kontrollen und Strafen von der Vergangenheit des Hinterziehers abhängig zu machen: Bei unverändertem Steueraufkommen (Ertrag der Steuerhinterziehung für den Steuerpflichtigen) können sie gegenüber dem statischen System der Steuersicherung Kontrollkosten sparen. Bei einem endogenen Einkommen sind bezüglich der Wirksamkeit von Kontrollen und Strafen Abstriche zu machen, wenn in der Ausgangssituation das gesamte Einkommen hinterzogen wird, d.h. wenn der Hinterzieher die Ecklösung wählt. Die Erklärung für die Wirksamkeit von Kontrollen und Strafen ist einfach: Es wurde ein Steuerpflichtiger angenommen, der (fast) aus- 
schließlich an dem Erwartungsnutzen seines Nettoeinkommens interessiert ist und daher auf Änderungen des durch Kontrollen und Strafen direkt beeinflußten Ertrages der Steuerhinterziehung in gewünschter Weise reagiert. Da Steuerpflichtige ohne Steuermoral im Zusammenhang mit der Steuerhinterziehung die wichtigste Personengruppe sind, ist die Wirksamkeit von Kontrollen und Strafen ein wichtiges Ergebnis.

Die Steuerpolitik war in den dargestellten Modellen auf drei Ebenen präsent: Betrachtet wurden Änderungen des Einkommensteuertarifs, eine Quellensteuer und der Ersatz des Einkommens durch den Konsum als Bemessungsgrundlage der direkten Besteuerung. Eine Erhöhung des marginalen Einkommensteuersatzes hat einen negativen Einkommenseffekt auf die hinterzogene Steuer, wenn man von einer abnehmenden absoluten Risikoaversion ausgeht: Das sichere Nettoeinkommen des Steuerpflichtigen sinkt, so daß er weniger geneigt ist, durch eine unrichtige Steuererklärung eine unsichere Position gegenüber der Finanzbehörde aufzubauen. In vielen Modellen waren jedoch gegenläufige, für einen positiven Zusammenhang zwischen Steuerbelastung und -hinterziehung sprechende Effekte zu finden. Sie stellen sich z.B. ein, wenn die mit dem Nettoeinkommen steigende Geldstrafe nach deutschem Recht verhängt wird, man eine Ansteckung zur Steuerhinterziehung zwischen den Steuerpflichtigen unterstellt, die Hinterzieher sehen, daß sie öffentliche Leistungen kürzen, oder ein endogenes Einkommen und gleichzeitig eine Ecklösung angenommen werden. Der Nettoeffekt eines höheren Steuersatzes ist im allgemeinen jedoch nicht bestimmbar.

Die Wirkung einer Quellensteuer ist vorhersagbar, wenn der Steuerpflichtige nicht sein gesamtes Einkommen hinterzieht. Die zentrale Größe für einen Hinterzieher ist die hinterzogene Steuer: Sie bestimmt, um welchen Betrag sein Konsum durch eine unrichtige Steuererklärung nach oben oder - wenn er "erwischt" wird in wieweit der Konsum nach unten von dem sicheren Nettoeinkommen abweicht. Die Steuer, die ihm an der Quelle abgezogen wird, kann der Steuerpflichtige nicht mehr verkürzen. Die Quellensteuer senkt daher die Steuer, die maximal hinterzogen werden kann, wenn man davon ausgeht, daß maximal das tatsächliche Einkommen bzw. bestimmte Teile des Einkommens (Zinserträge etwa) hinterzogen werden können. Ist der Satz der Quellensteuer nicht zu groß - beträgt sie z.B. nur $10 \%$-, betrifft den Hinterzieher diese Ānderung der Nebenbedingung seines Entscheidungsproblems nicht. Die Steuer an der Quelle gleicht er durch ein höheres hinterzogenes Einkommen aus, so daß sich das Aufkommen der Quellensteuer und die aus der Veranlagung zur Einkommensteuer resultierende Zahlung saldieren. Wenn der Steuerpflichtige in der Ausgangssituation die Ecklösung wählt, ist ihm diese Form der Umgehung der Quellensteuer versperrt. Mit einem endogenen Zinseinkommen kann er einer Quellensteuer auf die Zinserträge aller Anlagen nur ausweichen, wenn er weniger spart. Das Reaktion eines Steuerpflichtigen, der alle 
Zinsen hinterzieht, auf eine Erhöhung oder Einführung einer Quellensteuer kann jedoch nicht vorhergesagt werden.

Als dritte Variante der Steuerpolitik ist der Ersatz einer Einkommensteuer durch eine direkte Konsumsteuer analysiert worden. Unter einer Einkommensteuer behaupten die Sparer, sie erzielten keine Zinserträge, wenn sie davon augehen können, daß die Finanzbehörden das "Bankgeheimnis" respektieren, d.h. die Sparkonten privater Haushalte nicht ohne Verdacht aufdecken. Eine Konsumsteuer könnte dagegen mit dem Bankgeheimnis leben, solange den Finanzbehörden nur die Quellen der Ersparnisse bekannt werden. Zu hohe deklarierte Ersparnisse vermindern zwar zunächst die Konsumsteuerschuld eines Haushalts. In späteren Perioden muß er aber einen um so höheren Konsum versteuern, wenn er nicht durch die Inkonsistenz seiner Steuererklärungen auffallen und eine Prüfung provozieren will. Mit einem progressiven Konsumsteuertarif wird ein rationaler Steuerpflichtiger durch eine mehr oder weniger den Tatsachen entsprechende Deklaration seiner Ersparnis die marginale Steuerbelastung in den verschieden Perioden angleichen - ein Effekt, den man aus der Perspektive der Lebenszyklusmodelle eines Sparers nur als wünschenswert bezeichnen kann.

Der Einfluß der Steuerpolitik auf die Steuerhinterziehung läßt sich nur schwer in einem Satz zusammenfassen. Zu unterschiedlich sind die Auswirkungen der einzelnen Instrumente. Die Änderung des Einkommensteuertarifs und die Erhebung einer Quellensteuer haben zu überraschenden Ergebnissen geführt. Eine bestimmte Tendenz in bezug auf die Steuerhinterziehung ist nicht erkennbar. Daher ist man versucht zu sagen, daß von der Steuerpolitik nicht erwartet werden kann, die Steuerhinterziehung einzudämmen, und daß die Finanzpolitik sich auf zwar unpopuläre, aber wirksame Kontrollen und Strafen verlassen sollte. Nur bezüglich der Wahl der Bemessungsgrundlage der direkten Besteuerung ist eine Ausnahme von diesem negativen Urteil festzuhalten: Eine Kosumsteuer kann in Verbindung mit einer Prüfung der Konsistenz von Steuererklärungen die - unterstellten - negativen Wirkungen der Steuerhinterziehung in erwünschte Bahnen lenken.

\section{In wieweit soll man die Steuerhinterziehung eindämmen?}

Es gibt wirksame Mittel, um die Steuerhinterziehung zu begrenzen. Sind die Strafe und die Entdeckungswahrscheinlichkeit nur groß genug, läßt sie sich theoretisch sogar zum Verschwinden bringen, und es stellt sich die Farge, ob die Finanzpolitik von dieser Möglichkeit nicht Gebrauch machen sollte. Dieses normative Problem ist in dieser Arbeit nicht behandelt worden. Dennoch sollen kurze Hinweise zu der wichtigen Frage, wie weit der Fiskus bei der Verfolgung der Steuerhinterziehung 
gehen sollte, den Schluß dieser Arbeit bilden.

Hohe Strafen für die Steuerhinterziehung dürften von vielen als ungerecht empfunden werden. Statt Exempel zu statuieren die Entdeckungswahrscheinlichkeit zu erhöhen, kostet jedoch Geld. Es wäre daher naheliegend, die Entdeckungswahrscheinlichkeit so lange zu erhöhen, bis die Grenzkosten der Steuerfahndung gleich dem marginalen Steueraufkommen sind, das durch zusätzliche Kontrollen aufgebracht wird. Folgt man dieser Regel, läßt sich zeigen, daß die Reduktion der Steuerhinterziehung auf Null nicht wünschenswert ist, wenn die marginalen Kosten einer höheren Entdeckungswahrscheinlichkeit positiv sind (Baldry, 1984). Die angegebene Regel zur Bestimmung des Umfangs von Kontrollen ist jedoch wenig überzeugend, wie Shoup (1969, S. 433) ausführt: Die Erhebung von Steuern kann nicht mit einer Güter produzierenden Unternehmung verglichen werden, die die Produktion ausweitet, bis die Grenzkosten gleich den Grenzerlösen sind. Die Erhebung von Steuern stellt keine Produktion dar, sondern eine Umverteilung zwischen verschiedenen Gruppen von Steuerpflichtigen und/oder zwischen privaten und staatlichen Ausgaben.

Eine überzeugendere Regel zur Bestimmung des Umfangs von Kontrollen ist, die Grenzkosten der Kontrollen und die marginale Verringerung aller durch die Steuerhinterziehung verursachten Wohlfahrtsverluste anzugleichen (Sandmo, 1981, Slemrod/Yitzhaki, 1987). Das Problem bei dieser Regel ist die Identifizierung und Quantifikation der angesprochenen Wohlfahrtsverluste. Drei Arten werden in der Literatur genannt: Die Steuerhinterziehung führt (i) ex post zu einer ungleichmäßigen Besteuerung (Cowell, 1989), (ii) zu einer Fehlallokation von Ressourcen (Virmani, 1990), und (iii) sie setzt die (risikoaversen) Steuerpflichtigen der Unsicherheit eventueller Prüfungen aus, die diese - bei gleicher erwarteter Steuerbelastung lieber nicht tragen würden (Slemrod/Yitzhaki, 1987). Neben Wohlfahrtsverlusten sind jedoch auch Wohlfahrtsgewinne durch die Steuerhinterziehung denkbar. Sie kann, wie in Kapitel 5 gezeigt worden ist, zu einer gleichmäßigeren Besteuerung beitragen. Sie kann zu einer Verbesserung der Allokation führen (Wiegard, 1984). Schließlich ist die These vertreten worden, daß risikoaverse Steuerpflichtige (mit endogenem Einkommen) es vorziehen, bei gleichem erwarteten Steueraufkommen einen Anreiz zur Steuerhinterziehung zu erhalten, statt mit einem erwarteten Ertrag der Steuerhinterziehung von Null rechnen zu müssen (Weiss, 1976, dessen Ergebnis von Yitzhaki, 1987, jedoch nicht bestätigt werden konnte).

Die theoretische Erfassung der Wohlfahrtswirkungen der Steuerhinterziehung steht im Gegensatz zu der positiven Theorie der Steuerhinterziehung noch am Anfang. Man darf gespannt sein, in wieweit die Wohlfahrtsverluste die Wohlfahrtsgewinne der Steuerhinterziehung übersteigen. Eins sollte bei dieser Debatte jedoch nicht aus den Augen verloren werden: Die gesellschaftliche Bewertung der Steuerhinterziehung ist keine Konstante. Sie ändert sich von Zeit zu Zeit, so daß 
die Finanzpolitik im mehr oder weniger großem zeitlichen Abstand aufgerufen ist, das Ausmaß der Steuerhinterziehung zu beeinflussen. Es ist dann wichtig, Vorstellungen über die Wirkung der finanzpolitischen Instrumente zu haben, die die Entscheidungen eines Steuerhinterziehers berühren. 


\section{Anhang}

Die Anhänge sind nach den Kapiteln und Abschnitten nummeriert, auf die sie sich beziehen. Die Reihenfolge der Rechnungen richtet sich nach dem Gang der Argumentation im Text.

\section{Anhang 3.3.1}

$$
\begin{aligned}
\frac{\partial C^{*}}{\partial p}= & \Delta^{-1} R t(1+\alpha) q W^{\prime}(G)\left(W^{\prime}(G) R_{a}(P)-W^{\prime}(P) R_{a}(G)\right) \gtreqless 0 \\
\frac{\partial A}{\partial p}= & -\operatorname{tr} \frac{\partial C^{*}}{\partial p}+(1+\alpha) t H^{*}-(q-p \alpha) t \frac{\partial H^{*}}{\partial p} \\
= & -\operatorname{tr}\left[\tilde{\Delta}^{-1} R t(1+\alpha) q W^{\prime}(G)\left(W^{\prime}(G) R_{a}(P)-W^{\prime}(P) R_{a}(G)\right)\right] \\
& +(1+\alpha) t H^{*} \\
& -(q-p \alpha) t\left[\tilde { \Delta } ^ { - 1 } \left\{V^{\prime \prime}(C)\left(W^{\prime}(G)+W^{\prime}(P) \alpha\right)\right.\right. \\
& \left.\left.+R^{2}\left(q W^{\prime \prime}(G) W^{\prime}(P)+p W^{\prime \prime}(P) W^{\prime}(G)(1+\alpha)\right)\right\}\right] \gtreqless 0
\end{aligned}
$$

Wegen $D^{*}=r\left(l_{1}-C^{*}\right)-H^{*}$ gilt:

$$
\begin{aligned}
\frac{\partial D^{*}}{\partial p}= & -r \frac{\partial C^{*}}{\partial p}-\frac{\partial H^{*}}{\partial p} \\
= & -\tilde{\Delta}^{-1}\left[V^{\prime \prime}(C)\left(W^{\prime}(G)+W^{\prime}(P) \alpha\right)\right. \\
& +R(1+\alpha)\left\{(R-r t \alpha) p W^{\prime \prime}(P) W^{\prime}(G)\right. \\
& \left.\left.+(1+r) W^{\prime}(P) q W^{\prime \prime}(G)\right\}\right]>0
\end{aligned}
$$

wenn die schwache Bedingung $R-r t \alpha>0 \Leftrightarrow 1+r(1-t(1+\alpha))>0$ erfüllt ist.

\section{Anhang 3.3.2}

$$
\frac{\partial H^{*}}{\partial a}=\tilde{\Delta}^{-1} V^{\prime \prime}(C) q W^{\prime}(G)\left(R_{a}(G)-R_{a}(P)\right)>0
$$




$$
\frac{\partial C^{*}}{\partial a}=\tilde{\Delta}^{-1} R t q W^{\prime \prime}(G) p W^{\prime \prime}(P)(1+\alpha)^{2}>0
$$

Mit

$$
\tilde{\Delta}^{-1}=\Delta^{-1} / t=V^{\prime \prime}(C) E_{H H} / t+R^{2} t q W^{\prime \prime}(G) p W^{\prime \prime}(P)(1+\alpha)^{2}
$$

folgt $\partial C^{*} / \partial a<1 / R$.

$$
\begin{aligned}
\frac{\partial H^{+}}{\partial r}= & \frac{\partial H^{*}}{\partial r}+\frac{\partial a^{+}}{\partial r} \frac{\partial H^{*}}{\partial r} \\
= & -\tilde{\Delta}^{-1}(1-t) q W^{\prime}(G)\left(R_{a}(G)-R_{a}(P)\right) \\
& {\left[-V^{\prime \prime}(C)\left(l_{1}-C\right)+R\left(q W^{\prime}(G)+p W^{\prime}(P)\right)\right] } \\
& -\tilde{\Delta}^{-1}(1-t)\left(l_{1}-C\right) V^{\prime \prime}(C) q W^{\prime}(G)\left(R_{a}(G)-R_{a}(P)\right) \\
= & -\tilde{\Delta}^{-1}(1-t) q W^{\prime}(G)\left(R_{a}(G)-R_{a}(P)\right) \\
& R\left(q W^{\prime}(G)+p W^{\prime}(P)\right)>0
\end{aligned}
$$

Der Substitutionseffekt $\partial C^{+} / \partial r>0$ kann mit demselben Verfahren abgeleitet werden.

\section{Anhang 3.4}

$$
\begin{gathered}
\frac{\partial C^{*}}{\partial \alpha}=-\Delta^{-1} \operatorname{trp}\left[W^{\prime}(P)+(1+r(1-t(1+\alpha))) W^{\prime \prime}(P)\left(l_{1}-C\right)\right] \gtreqless 0 \\
\frac{\partial C^{*}}{\partial t}=-\Delta^{-1}\left[-(1+r) q W^{\prime \prime}(G)\left(-w_{2}-(1+r) w_{1}\right)\right. \\
+r(1+\alpha) p W^{\prime}(P) \\
-(1+r(1-t(1+\alpha))) p W^{\prime \prime}(P) \\
\left.\left(-w_{2}-(1+r(1-t(1+\alpha))) w_{1}-r(1+\alpha)\left(l_{1}-C\right)\right)\right] \gtreqless 0 \\
\left.\frac{\partial C^{*}}{\partial \alpha}\right|_{q-p \alpha=k}=\frac{\partial C^{*}}{\partial \alpha}+\frac{\partial C^{*}}{\partial p} \frac{\partial p}{\partial \alpha} \\
=-\Delta^{-1}\left[p t r(1+r(1-t(1+\alpha))) W^{\prime \prime}(P)\left(l_{1}-C\right)\right. \\
\left.-(1+r) \frac{p}{1+\alpha}\left(W^{\prime}(G)-W^{\prime}(P)\right)\right] \gtreqless 0 \\
\frac{\partial H^{*}}{\partial r}=\frac{\partial r\left(l_{1}-C^{*}\right)}{\partial r}=\left(l_{1}-C^{*}\right) \frac{\Delta}{\Delta}-r \frac{\partial C^{*}}{\partial r}
\end{gathered}
$$




$$
\begin{aligned}
= & \Delta^{-1}\left[( l _ { 1 } - C ) \left\{V^{\prime \prime}(C)+q(1+r) W^{\prime \prime}(G)\right.\right. \\
& \left.+p(1+r(1-t(1+\alpha))) W^{\prime \prime}(P)\right\} \\
& \left.-r q W^{\prime}(G)-r p(1-t(1+\alpha)) W^{\prime}(P)\right]>0
\end{aligned}
$$

wenn die (hinreichende) Bedingung $1-t(1+\alpha) \geqq 0$ erfüllt ist.

\section{Anhang 3.5}

Dieser Anhang enthält noch eine Ergebnisformeln zu Tabelle 3.1 in Abschnitt 3.5. Die in der Tabelle angegebenen unbestimmten Ergebnisse werden nicht weiter dokumentiert, da sie für einige Parameteränderungen schon exemplarisch abgeleitet worden sind.

Bei einer inneren Lösung ändert sich $S^{*}=t H^{*}$ wie folgt:

$$
\frac{\partial S^{*}}{\partial t}=\frac{-\left(E_{C C} E_{S t}-E_{C t} E_{S C}\right)}{E_{C C} E_{S S}-E_{C H}^{2}}
$$

Der Nenner ist positiv. Der Zähler ist gleich

$$
V^{\prime \prime}(C) q W^{\prime}(G)\left(R_{a}(G)-R_{a}(P)\right)\left(-w_{2}-R w_{1}-r\left(l_{1}-C\right)\right)<0
$$

Damit ist auch

$$
\frac{\partial S^{*}}{\partial t}=H^{*}+t \frac{\partial H^{*}}{\partial t}<0
$$

Daraus folgt sofort $\partial H^{*} / \partial t<0$.

\section{Anhang 3.7}

$$
\begin{aligned}
\frac{\partial S^{*}}{\partial p}= & \Delta^{-1}\left[\left\{V^{\prime \prime}\left(C^{*}\right)+\left(1+r N^{\prime}\right)^{2}\left(q W^{\prime \prime}(G)+p W^{\prime \prime}(P)\right)\right.\right. \\
& \left.\left.+r^{2} N^{\prime \prime}\left(q W^{\prime}(G)+p W^{\prime}(P)\right)\right\}\left(W^{\prime}(G)+W^{\prime}(P) \alpha\right)\right] \\
& \left.+\left(1+r N^{\prime}\right)^{2}\left(W^{\prime}(G) p W^{\prime \prime}(P)+W^{\prime}(P) q W^{\prime \prime}(G)\right)(1+\alpha)\right]<0 \\
\frac{\partial S^{*}}{\partial \alpha}=- & -\Delta^{-1}\left[\left\{V^{\prime \prime}\left(C^{*}\right)+r^{2} N^{\prime \prime}\left(q W^{\prime}(G)+p W^{\prime}(P)\right)\right\}\left(-p W^{\prime}(P)+p W^{\prime \prime}(P) \alpha S\right)\right. \\
& +\left(1+r N^{\prime}\right)^{2}\left\{-p W^{\prime}(P)\left(q W^{\prime \prime}(G)+p W^{\prime \prime}(P)\right)\right. \\
& \left.\left.+p W^{\prime \prime}(P) S q W^{\prime \prime}(G)(1+\alpha)\right\}\right]<0
\end{aligned}
$$




\section{Anhang 5.3.1}

\section{Steuerbemessungsgrundlagen inklusive und exklusive der Steuern selbst bei progressivem Tarif}

In der Bemessungsgrundlage der Einkommensteuer ist die Steuer selbst enthalten. Ein Stpfl. kann das zu versteuernde Einkommen nicht um die später zu zahlende Steuer vermindern. Eine direkte Konsumsteuer hat dagegen laut ihrem Namen eine Bemessungsgrundlage, in der die zu zahlende Steuer nicht enthalten ist. Nun ist bereits bekannt, daß die Wahl zwischen einer Bemessungsgrundlage inklusive und exklusive der Steuer unter einem proportionalen Tarif gleichgültig ist. Stimmen der durchschnittliche und der marginale Steuersatz stets überein, kann man durch eine geeignete Wahl der Steuersätze eine identische Belastung bei beiden Bemessungsgrundlagen erreichen. Dies sei für eine direkte Konsumsteuer kurz gezeigt. Es gilt natürlich in gleicher Weise für eine proportionale Einkommensteuer.

Ist $Y$ das Einkommen und $S$ die Ersparnis eines Stpfl., so ergibt sich sein Konsum $C$ unter einer Konsumsteuer auf der Basis exklusive der Steuern selbst mit dem Satz $t_{e}$ aus der Gleichung

$$
\begin{gathered}
C=Y-S-t_{e} C \\
\Leftrightarrow \quad C=\frac{1}{1+t_{e}}(Y-S)=Y-S-\frac{t_{e}}{1+t_{e}}(Y-S)
\end{gathered}
$$

Man kan also auch das Einkommen abzüglich der Ersparnis $Y-S$, von dem der Stpfl. seinen Konsum und seine Steuerzahlungen bestreiten muß, als Bemessungsgrundlage einer proportionalen Steuer mit dem Satz

$$
t_{i}=\frac{t_{e}}{1+t_{e}}
$$

wählen, ohne daß der Steuerzahler gegenüber der Situation mit der steuerexklusiven Basis schlechter gestellt wäre (Meade, 1978, S. 28 f.). ( $C$ und $S$ können auch als deklarierte statt tatsächliche Größen interpretiert werden).

Im folgenden geht es um die Frage, ob auch bei progressiven Steuerbetragsfunktionen die Entscheidung zwischen steuerin- und exklusiven Bemessungsgrundlagen gleichgültig ist. Die Antwort auf diese Frage ist positiv. Dieses Ergebnis erlaubt es, in der Analyse von Steuerwirkungen mit der Basis zu arbeiten, die am einfachsten zu handhaben ist. Die folgenden zwei Abschnitte dieses Anhangs behandeln jeweils den indirekt und den direkt progressiven Tarif.

\section{Indirekt progressiver Tarif}

Ein indirekt progressiver Tarif hat einen konstanten marginalen und einen steigenden durchschnittlichen Steuersatz. Aus der indirekt progressiven Konsumsteuer 
mit dem Freibetrag $f_{e}$ und der Steuerermäßigung $a_{e}$

$$
K(C)=t_{e}\left(C-f_{e}\right)-a_{e}
$$

läßt sich explizit eine Steuer mit dem gleichen Aufkommen auf der Basis des Einkommens abzüglich der Ersparnis ableiten. Wir gehen von der Gleichung

$$
C+t_{e}\left(C-f_{e}\right)-a_{e}=Y-S
$$

aus und lösen sie nach $C$ auf.

$$
\begin{gathered}
C=Y-S-\frac{t_{e}}{1+t_{e}}\left(Y-S-f_{e}\right)+\left(1-\frac{t_{e}}{1+t_{e}}\right) a_{e} \\
\Leftrightarrow \quad C=Y-S-t_{i}\left(Y-S-f_{i}\right)+a_{i}
\end{gathered}
$$

wobei

$$
t_{i}=\frac{t_{e}}{1+t_{e}}
$$

$a_{i}=\left(1-t_{i}\right) a_{e}$ und $f_{i}=f_{e}$ der Steuersatz, die Steuerermäßigung und der Freibetrag des steuerinklusiven Tarifs sind.

\section{Direkt progressiver Tarif}

Der direkt progressive Tarif zeichnet sich durch steigende marginale und durchschnittliche Steuersätze aus. Betrachten wir den Tarif $K(C)$ mit den Eigenschaften

$$
\begin{gathered}
\infty>K(C)>0 \\
\infty>K^{\prime}(C)>0 \\
K^{\prime \prime}(C)>0
\end{gathered}
$$

für den Bereich $C \geqq z$ und

$$
K(C)_{\mid C<z}=0
$$

$K^{\prime}(C)<\infty$ bedeutet, daß ein zusätzlicher Konsum nicht unermeßlich hohe zusätzliche Konsumsteuern verursacht bzw. daß zusätzliches Einkommen - gesetzt den Fall, es wird nicht gespart - zu einem Teil konsumiert werden kann und nicht voll vom Fiskus vereinnahmt wird. Belastet wird ein Konsum $C \geqq z$.

Welche Eigenschaften hat ein direkt progressiver Tarif $T(Y-S)$ auf der steuerinklusiven Basis $Y-S$, der bei gegebenem Einkommen und gegebener Ersparnis stets das gleiche Aufkommen liefert wie $K(C)$ ? Wir gehen von der Gleichung $C+K(C)=Y-S=Q$ aus und fordern $T(Q)=K(C)$. Einsetzen ergibt

$$
T(C+K(C))=K(C)
$$


Durch die Differenzierung dieser Identität gelangt man zu Aussagen über die Eigenschaften der Steuerfunktion $T(Q)$.

$$
\begin{array}{r}
T^{\prime}(C+K(C))\left(1+K^{\prime}(C)\right)=K^{\prime}(C) \\
\Leftrightarrow \quad T^{\prime}=\frac{K^{\prime}}{1+K^{\prime}}>0,<1
\end{array}
$$

$\mathrm{DaB}$ der marginale Steuersatz kleiner als eins ist, ergibt sich aus $K^{\prime}<\infty$. Wenn die Steuer also bei einer steuerexklusiven Basis nicht konfiskatorisch ist, so ist sie dies auch bei der steuerinklusiven Bemessungsgrundlage nicht. Die zweite Ableitung von Gleichung (*) erlaubt eine Aussage über $T^{\prime \prime}$ :

$$
\begin{gathered}
T^{\prime \prime}\left(1+K^{\prime}\right)^{2}+T^{\prime} K^{\prime \prime}=K^{\prime \prime} \\
\Leftrightarrow \quad T^{\prime \prime}=\frac{\left(1-T^{\prime}\right) K^{\prime \prime}}{\left(1+K^{\prime}\right)^{2}}=\frac{K^{\prime \prime}}{\left(1+K^{\prime}\right)^{3}}>0
\end{gathered}
$$

Aus dem progressivem Verlauf des Tarifs auf der steuerexklusiven Basis folgt, daß auch der belastungsgleiche steuerinklusive Tarif progressiv ist.

Auch die Umkehrung dieser Aussage gilt. Durch Differenzieren von $K(C)=$ $K(Q-T(Q))=T(Q)$ erhalten wir

$$
K^{\prime}=\frac{T^{\prime}}{1-T^{\prime}}>0
$$

und

$$
K^{\prime \prime}=\frac{\left(1+K^{\prime}\right) T^{\prime \prime}}{\left(1-T^{\prime}\right)^{3}}=\frac{T^{\prime \prime}}{\left(1-T^{\prime}\right)^{3}}>0
$$

Schließlich soll noch festgestellt werden, ob beide Steuerfunktionen denselben Bereich freistellen. $K(C)_{\mid C<z}=0$ bedeutet $C=Q$ wenn $C<z$. Also gilt auch $Q=C$, wenn $Q<z$, und folglich $T(Q)_{\mid Q<z}=0$.

Wir haben die zwei nichtlinearen belastungsgleichen Tarife untersucht und gesehen, daß sie entsprechende Eigenschaften haben. Ein Beispiel soll den Schluß dieses Anhangs bilden. Sei $T(Q)=Q-a Q^{b}$ mit $0<a, b<1$ der steuerexklusive Tarif für den Bereich $Q \geqq 1$ (mit $Q<1$ kann diese Steuerfunktion regressiv sein). Es gilt

$$
\begin{aligned}
C & =Q-T(Q)=a Q^{b} \\
\Leftrightarrow \quad\left(\frac{1}{a} C\right)^{1 / b} & =Q
\end{aligned}
$$

Der steuerexklusive Tarif $K(C)$, der $K(C)=T(Q)$ erfüllt, ist schnell gefunden:

$$
K(C)=T(Q)=Q-a Q^{b}=\left(\frac{1}{a} C\right)^{1 / b}-C
$$

Er gilt für den Bereich $C \geqq 1$. Die Überführung eines in funktionaler Form vorliegenden Tarifs in die belastungsgleiche Steuerbetragsfunktion mit der alternativen Bemessungsgrundlage ist jedoch nicht immer so einfach wie in diesem Beispiel. 


\section{Anhang 5.3.2}

Der Vorteil $V I P_{2}$ der interperiodischen Durchschnittsbildung des Konsums - gemessen in Einheiten des Konsums in Periode 2 - ist gleich dem zusätzlichen $C_{2}$ bei gleichem $C_{1}$ unter beiden Regimes.

$$
V I P_{2}=w_{2}+(1+r)\left(w_{1}-C_{1}\right)-(2+r) T\left(\frac{L_{2}}{2+r}\right)-\widetilde{C}_{2}
$$

Dabei ist $\widetilde{C}_{\boldsymbol{i}}$ der Konsum ohne die Durchschnittsbildung. $\widetilde{S}$ sei die Ersparnis ohne Progressionsausgleich. Definitionsgemäß ist

$$
\tilde{C}_{2}=w_{2}+(1+r) \tilde{S}+T\left(w_{2}+(1+r) \tilde{S}\right)
$$

Laut Annahme gilt

$$
C_{1}=\widetilde{C}_{1}=w_{1}-\widetilde{S}-T\left(w_{1}-\tilde{S}\right)
$$

Setzt man die zwei letzten Gleichungen in $V I P_{2}$ ein, ergibt sich

$$
V I P_{2}=(1+r) T\left(w_{1}-\tilde{S}\right)+T\left(w_{2}+(1+r) \tilde{S}\right)-(2+r) T\left(\frac{L_{2}}{2+r}\right)
$$

Ausgedrückt in Einheiten von $C_{1}$ ist der Vorteil des Progressionsausgleichs

$$
V I P_{1}=\frac{1}{1+r} V I P_{2}
$$

In Abbildung 2 ist z.B. die Strecke $D B$ gleich $(1+r)^{-1}$ mal der Strecke $A B$.

\section{Anhang 5.4.2}

Das Problem

$$
\begin{array}{cl}
\max _{S, D} & u U\left(C_{1}, C_{2}\right)+(1-u) U\left(C_{1}, 0\right) \\
\text { u.d.N. } & C_{1}=w_{1}-S-T\left(w_{1}-D\right) \\
& C_{2}=w_{2}+(1+r) S-T\left(w_{2}+(1+r) D\right)
\end{array}
$$

mit $D=S+F$ als deklarierte Ersparnis führt zu den Optimumbedingungen

$$
\begin{gathered}
-u U_{1}\left(C_{1}, C_{2}\right)-(1-u) U_{1}\left(C_{1}, 0\right)+u U_{2}\left(C_{1}, C_{2}\right)(1+r)=0 \\
\left(u U_{1}\left(C_{1}, C_{2}\right)+(1-u) U_{1}\left(C_{1}, 0\right)\right) T^{\prime}\left(B_{1}\right)-u U_{2}\left(C_{1}, C_{2}\right)(1+r) T^{\prime}\left(B_{2}\right)=0
\end{gathered}
$$

Man beachte, daß im Todesfall $d C_{2}=0$ vorgegeben ist und daher die Ableitung der Nutzenfunktion nach $C_{2}$ in diesem Zustand nicht auftaucht. Dividiert man die zweite durch die erste Bedingung, ergibt sich $T^{\prime}\left(B_{1}\right)=T^{\prime}\left(B_{2}\right)$. 


\section{Anhang 6.2.3 a}

$$
\begin{aligned}
\Delta E^{x} & =E^{x+1}-E^{x} \\
& =\frac{\sum_{i=0}^{x} h_{i}(q \eta)^{i}}{(1-\eta) \sum_{i=0}^{x+1}(q \eta)^{i}}-\frac{\sum_{i=0}^{x-1} h_{i}(q \eta)^{i}}{(1-\eta) \sum_{i=0}^{x}(q \eta)^{i}} \\
& =\frac{\sum_{i=0}^{x} h_{i}(q \eta)^{i} \sum_{i=0}^{x}(q \eta)^{i}-\sum_{i=0}^{x-1} h_{i}(q \eta)^{i} \sum_{i=0}^{x+1}(q \eta)^{i}}{(1-\eta) \sum_{i=0}^{x+1}(q \eta)^{i} \sum_{i=0}^{x}(q \eta)^{i}}
\end{aligned}
$$

Der Nenner dieses Ausdrucks ist positiv. Den Zähler kann man wie folgt schreiben:

$$
\begin{aligned}
& \sum_{i=0}^{x-1} h_{i}(q \eta)^{i}\left[\sum_{i=0}^{x}(q \eta)^{i}-\sum_{i=0}^{x+1}(q \eta)^{i}\right]+h_{x}(q \eta)^{x} \sum_{i=0}^{x}(q \eta)^{i} \\
= & h_{0}\left[(q \eta)^{x}-\left((q \eta)^{x}+(q \eta)^{x+1}\right)\right] \\
& +h_{1} q \eta\left[(q \eta)^{x-1}+(q \eta)^{x}-\left((q \eta)^{x-1}+(q \eta)^{x}+(q \eta)^{x+1}\right)\right] \\
& +\cdots \cdots \\
& +h_{x-1}(q \eta)^{x-1}\left[\sum_{i=1}^{x}(q \eta)^{i}-\sum_{i=1}^{x+1}(q \eta)^{i}\right] \\
& +h_{x}(q \eta)^{x} \sum_{i=0}^{x}(q \eta)^{i} \\
= & (q \eta)^{x}\left\{h_{0}+\left(h_{1}-h_{0}\right)(1+q \eta)+\ldots+\left(h_{x}-h_{x-1}\right) \sum_{i=0}^{x}(q \eta)^{i}\right\}
\end{aligned}
$$

Wegen $h_{x}-h_{x-1}=-p \pi \eta^{-x}$ nimmt der Zähler von $\Delta E^{x}$ folgende im Text angegebene Form an:

$$
(q \eta)^{x}\left[1-p \pi \sum_{i=0}^{x} \eta^{-i} \sum_{j=0}^{i}(q \eta)^{j}\right]
$$

Es soll nun die zweite Differenz $\Delta E^{x+1}-\Delta E^{x}$ berechnet werden:

$$
\begin{gathered}
\Delta E^{x+1}-\Delta E^{x}= \\
\frac{(q \eta)^{x+1}\left[1-p \pi \sum_{i=0}^{x+1} \eta^{-i} \sum_{j=0}^{i}(q \eta)^{j}\right]}{(1-\eta) \sum_{i=0}^{x+2}(q \eta)^{i} \sum_{i=0}^{x+1}(q \eta)^{i}}-\frac{(q \eta)^{x}\left[1-p \pi \sum_{i=0}^{x} \eta^{-i} \sum_{j=0}^{i}(q \eta)^{j}\right]}{(1-\eta) \sum_{i=0}^{x+1}(q \eta)^{i} \sum_{i=0}^{x}(q \eta)^{i}}
\end{gathered}
$$




$$
\begin{gathered}
=\left[(1-\eta) \sum_{i=0}^{x+2}(q \eta)^{i} \sum_{i=0}^{x+1}(q \eta)^{i} \sum_{i=0}^{x}(q \eta)^{i}\right]^{-1} \times \\
(q \eta)^{x}\left[\sum_{i=1}^{x+1}(q \eta)^{i}\left\{1-p \pi \sum_{i=0}^{x+1} \eta^{-i} \sum_{j=0}^{i}(q \eta)^{j}\right\}-\sum_{i=0}^{x+2}(q \eta)^{i}\left\{1-p \pi \sum_{i=0}^{x} \eta^{-i} \sum_{j=0}^{i}(q \eta)^{j}\right\}\right]
\end{gathered}
$$

In dem Zähler dieses Ausdrucks heben sich bestimmte Terme auf, so daß er folgende Form annimmt:

$$
(q \eta)^{x}\left[-\sum_{i=1}^{x+1}(q \eta)^{i}\left\{p \pi \eta^{-(x+1)} \sum_{j=0}^{x+1}(q \eta)^{j}\right\}-\left(1+(q \eta)^{x+2}\right)\left\{1-p \pi \sum_{i=0}^{x} \eta^{-i} \sum_{j=0}^{i}(q \eta)^{j}\right\}\right]
$$

Man sieht nun: $\Delta E^{x+1}-\Delta E^{x}$ ist negativ, solange $\Delta E^{x}$ positiv ist.

\section{Anhang 6.2.3 b}

Den Kapitalwert des Hinterziehers, der nach $x$ Erfolgen ehrlich wird, kann man wie folgt entwickeln, wenn man $h_{i}$ substituiert:

$$
\begin{aligned}
E^{x} & =\frac{\sum_{i=0}^{x-1} h_{i}(q \eta)^{i}}{(1-\eta) \sum_{i=0}^{x}(q \eta)^{i}} \\
& =\frac{\sum_{i=0}^{x-1}(q \eta)^{i}\left(1-p \pi \sum_{j=0}^{i} \eta^{-j}\right)}{(1-\eta) \sum_{i=0}^{x}(q \eta)^{i}} \\
& =\frac{\frac{1-(q \eta)^{x}}{1-q \eta}-p \pi \sum_{i=0}^{x-1}(q \eta)^{i} \frac{1-\eta^{-(i+1)}}{1-\eta^{-1}}}{(1-\eta) \frac{1-(q \eta)^{x+1}}{1-q \eta}} \\
= & \frac{\frac{1-(q \eta)^{x}}{1-q \eta}-\frac{p \pi}{1-\eta^{-1}}\left[\frac{1-(q \eta)^{x}}{1-q \eta}-\frac{1-q^{x}}{\eta(1-q)}\right.}{(1-\eta) \frac{1-(q \eta)^{x+1}}{1-q \eta}}
\end{aligned}
$$

Mit $x \rightarrow \infty$ ergibt sich:

$$
\lim _{x \rightarrow \infty} E^{x}=\frac{1-\frac{p \pi \eta}{\eta-1}\left(1-\frac{1-q \eta}{\eta(1-q)}\right)}{1-\eta}
$$




$$
\begin{aligned}
& =\frac{1-\frac{\pi}{\eta-1}(\eta-1)}{1-\eta} \\
& =\frac{-\alpha}{1-\eta}
\end{aligned}
$$

\section{Anhang 6.2.3 c}

Gezeigt wird (i) $\Delta E^{x} \gtreqless 0 \Leftrightarrow E^{x} \lesseqgtr N^{x}$. Das Vorzeichen von $\Delta E^{x}$ bestimmt der Zähler von Gleichung (*) aus Anhang 6.2.3 a. Vergleichen wir nun $E^{x}$ und $N^{x}$. Dazu wird $N^{x}$ zunächst in eine andere Form gebracht:

$$
\begin{aligned}
N^{x} & =\frac{1-p \pi \sum_{i=0}^{x} q^{i}}{1-\eta} \\
& =\frac{1-p \pi-p \pi \sum_{i=1}^{x} \eta^{-i}(q \eta)^{i}}{1-\eta} \\
& =\frac{1-p \pi+\sum_{i=1}^{x}\left(h_{i}-h_{i-1}\right)(q \eta)^{i}}{1-\eta} \\
& =\frac{\sum_{i=0}^{x} h_{i}(q \eta)^{i}-\sum_{i=1}^{x} h_{i-1}(q \eta)^{i}}{1-\eta}
\end{aligned}
$$

Dabei ist die Beziehung $h_{i}-h_{i-1}=-p \pi \eta^{-i}$ ausgenutzt worden.

Es gilt nun

$$
\begin{gathered}
E^{x} \lesseqgtr N^{x} \\
\Leftrightarrow \frac{\sum_{i=0}^{x-1} h_{i}(q \eta)^{i}}{(1-\eta) \sum_{i=0}^{x}(q \eta)^{i}} \lesseqgtr \frac{\sum_{i=0}^{x} h_{i}(q \eta)^{i}-\sum_{i=1}^{x} h_{i-1}(q \eta)^{i}}{1-\eta} \\
\Leftrightarrow 0 \lesseqgtr \sum_{i=0}^{x} h_{i}(q \eta)^{i} \sum_{i=0}^{x}(q \eta)^{i}-\sum_{i=1}^{x} h_{i-1}(q \eta)^{i} \sum_{i=0}^{x}(q \eta)^{i}-\sum_{i=0}^{x-1} h_{i}(q \eta)^{i}
\end{gathered}
$$

Die zwei letzten Ausdrücke auf der rechten Seite dieser Ungleichung kann man zusammenfassen, so daß die Ungleichung folgendes Aussehen annimmt:

$$
0 \lesseqgtr \sum_{i=0}^{x} h_{i}(q \eta)^{i} \sum_{i=0}^{x}(q \eta)^{i}-q \eta \sum_{i=0}^{x-1} h_{i}(q \eta)^{i} \sum_{i=0}^{x}(q \eta)^{i}-\sum_{i=0}^{x-1} h_{i}(q \eta)^{i}
$$




$$
\begin{aligned}
& \Leftrightarrow 0 \lesseqgtr \sum_{i=0}^{x} h_{i}(q \eta)^{i} \sum_{i=0}^{x}(q \eta)^{i}-\sum_{i=0}^{x-1} h_{i}(q \eta)^{i}\left[\sum_{i=1}^{x+1}(q \eta)^{i}+1\right] \\
& \Leftrightarrow 0 \lesseqgtr \sum_{i=0}^{x} h_{i}(q \eta)^{i} \sum_{i=0}^{x}(q \eta)^{i}-\sum_{i=0}^{x-1} h_{i}(q \eta)^{i} \sum_{i=0}^{x+1}(q \eta)^{i}
\end{aligned}
$$

Vergleicht man (**) und den Zähler von (*) aus Anhang 6.2.3 a, folgt die behauptete Äquivalenz (i).

Es wird jetzt gezeigt, daß auch die Beziehung (ii) $E^{x} \lesseqgtr N^{x} \Leftrightarrow E^{x+1} \lesseqgtr N^{x}$ gilt. Dazu vergleichen wir $E^{x+1}$ und $N^{x}$ :

$$
\begin{aligned}
& E^{x+1} \lesseqgtr N^{x} \\
\Leftrightarrow & \frac{\sum_{i=0}^{x} h_{i}(q \eta)^{i}}{(1-\eta) \sum_{i=0}^{x+1}(q \eta)^{i}} \lesseqgtr \frac{\sum_{i=0}^{x} h_{i}(q \eta)^{i}-\sum_{i=1}^{x} h_{i-1}(q \eta)^{i}}{1-\eta} \\
\Leftrightarrow & 0 \lesseqgtr \sum_{i=0}^{x} h_{i}(q \eta)^{i} \sum_{i=0}^{x+1}(q \eta)^{i}-\sum_{i=1}^{x} h_{i-1}(q \eta)^{i} \sum_{i=0}^{x+1}(q \eta)^{i}-\sum_{i=0}^{x} h_{i}(q \eta)^{i} \\
\Leftrightarrow & 0 \lesseqgtr \sum_{i=0}^{x} h_{i}(q \eta)^{i} \sum_{i=1}^{x+1}(q \eta)^{i}-q \eta \sum_{i=0}^{x-1} h_{i}(q \eta)^{i} \sum_{i=0}^{x+1}(q \eta)^{i} \\
\Leftrightarrow & 0 \lesseqgtr \sum_{i=0}^{x} h_{i}(q \eta)^{i} \sum_{i=0}^{x}(q \eta)^{i}-\sum_{i=0}^{x-1} h_{i}(q \eta)^{i} \sum_{i=0}^{x+1}(q \eta)^{i}
\end{aligned}
$$

Diese Ungleichung entspricht der Ungleichung (**), so daß die Äquivalenz (ii) folgt.

\section{Anhang 6.3.2}

Mit $p_{1}=p_{2}=p, \alpha_{1}=\alpha_{2}=\alpha$ und $1-p(1+\alpha)>0$ ergibt sich aus $(6.17)$ die Ungleichung

$$
p^{2}-p \frac{1-\eta}{\eta} \gtreqless\left(\frac{1-\eta}{\eta}\right)^{2}
$$

Die quadratische Ergänzung ergibt

$$
\left(p-\frac{1-\eta}{2 \eta}\right)^{2} \gtreqless\left(\frac{1-\eta}{2 \eta}\right)^{2}+\left(\frac{1-\eta}{\eta}\right)^{2}=\frac{5(1-\eta)^{2}}{4 \eta^{2}}
$$

Ziehen wir auf beiden Seiten die Wurzel:

$$
p \gtreqless \frac{1-\eta}{2 \eta} \pm \frac{5^{1 / 2}(1-\eta)}{2 \eta}
$$


Da eine Wahrscheinlichkeit nicht negativ ist, kann die negative Wurzel vernachlässigt werden. Damit ist

$$
p \gtreqless \frac{(1-\eta)\left(1+5^{1 / 2}\right)}{\eta 2}
$$

die Bedingung für $E^{100} \gtreqless E^{110}$. 


\section{Symbolverzeichnis (Auswahl)}

$A$ erwartetes Steueraufkommen

$B$ Steuerbemessungsgrundlage

C Konsum

$D$ deklariertes Einkommen

$E \quad$ Erwartungswertoperator

$E \quad$ Barwert der Erträge der periodischen Steuerhinterziehung (Kapitel 6)

$F \quad$ fiktive Ersparnis

$G \quad$ Nettoeinkommen des Stpfl., wenn er nicht entdeckt wird

$G_{i} \quad$ Gruppe i (Kapitel 6)

$H$ hinterzogenes Einkommen

K Budget der Finanzbehörde

$L \quad$ Lagrangefunktion

$N$ Barwert der Erträge der notorischen Steuerhinterziehung (Kapitel 6)

$P \quad$ Nettoeinkommen des Stpfl., wenn er entdeckt wird

$R_{a} \quad$ absolute Risikoaversion

$R=1+r(1-t)$

$S$ hinterzogene Steuer

$S$ Ersparnis (Kapitel 5)

$T \quad$ Steuertarif

$U \quad$ Nutzenfunktion 
$Y \quad$ Bruttoeinkommen des Stpfl.

a Steuerermäßigung

b Quellensteuersatz

$h \quad$ Arbeitszeit

$h_{i} \quad$ erwarteter Ertrag der Steuerhinterziehung (Kapitel 6)

$k \quad$ Kosten der Prüfung der letzten Steuererklärung

$l_{i} \quad$ exogenes Einkommen nach Steuer

p Entdeckungswahrscheinlichkeit

$q \quad=1-p$

$r \quad$ Zinssatz

$s$ exogene Ersparnis

$t$ Steuersatz

$t$ Periode (Kapitel 6)

$v \quad$ Verjährungsfrist für die Steuerhinterziehung

$w_{i} \quad$ exogenes Einkommen

$\alpha \quad$ Parameter der Strafe $\Phi_{1}$

$\beta \quad$ Parameter der Strafe $\Phi_{2}$

$\gamma \quad$ Parameter der Strafe $\Phi_{3}$

$\eta$ Diskontfaktor des Stpfl.

$\theta k \quad$ zusätzliche Kosten einer rückwirkenden Kontrolle

$\lambda$ Lagrange-Multiplikator

$\mu \quad$ Lagrange-Multiplikator

$\pi \quad=1+\alpha$ 


\section{Literaturverzeichnis}

Allingham, M.G. und A. Sandmo, 1972, Income tax evasion - a theoretical analysis, Journal of Public Economics 1, S. 323 - 338.

Andersen, P., 1977, Tax evasion and labor supply, Scandinavian Journal of Economics 79, S. 375 - 383.

Arrow, K.J., 1970, Essays in the theory of risk bearing, Amsterdam.

Atkinson, A.B. und J.E. Stiglitz, 1980, Lectures on public economics, London u.a.O.

Baldry, J.C., 1979, Tax evasion and labour supply, Economic Letters 3, S. 53 -56 .

Baldry, J.C., 1984, The enforcement of income tax laws: efficiency implications, Economic Record 60, S. 156 - 159.

Bamberg, G. und W.F. Richter, 1988, Risk-taking under progressive taxation - three partial effects, in: W. Eichhorn (Hrsg.), Measurement in economics, Heidelberg, S. $479-497$.

Baumol, W.J., 1972, Economic theory and operations analysis, Englewood Cliffs (1. Aufl. 1961).

Bilsdorfer, P., 1989, Die Informationsquellen und -wege der Finanzverwaltung: Wege und Methoden der Auskunftserlangung im In- und Ausland zum Zwecke der Besteuerung, Bielefeld (2. Aufl.).

Bradford, D.F. und U.S. Treasury Tax Policy Staff, 1984, Blueprints for basic tax reform, Washington (2. Aufl.; zuerst publiziert als U.S. Treasury Department, 1977).

Bradford, D.F., 1986, Untangling the income tax, Cambridge (Ma.) und London.

Braulke, M. und H. Sauermann, 1986, Die Firma im Halbschatten, Finanzarchiv N.F. 44, S. $297-305$.

Bundesgerichtshof, 1989, Urteil vom 20.6.1989 VIII R 82/86, in: Bundessteuerblatt 39 , S. $836-846$. 
Bundesministerium der Finanzen, 1986, Besteuerung der Kapitaleinkünfte, Bericht des BMF für den Rechnungsprüfungsausschuß des Deutschen Bundestages, in: Presse- und Informationsamt der Bundesregierung (Hrsg.), Aktuelle Beiträge zur Wirtschafts- und Finanzpolitik 42.

Bundesrechnungshof, 1985, Bemerkungen des Bundesrechnungshofes 1985 zur Haushalts- und Wirtschaftsführung, Bundestagsdrucksache 10/4367.

Cameron, S., 1988, The economics of crime deterrence: a survey of theory and evidence, Kyklos 41, S. $301-323$.

Christiansen, V., 1980, Two comments on tax evasion, Journal of Public Economics 13 , S. $389-393$.

Clotfelter, C.T., 1983, Tax evasion and tax rates: an analysis of individual returns, Review of Economics and Statistics 65, S. 363 - 373.

Cook, P.J., 1972, A "oneline" proof of the Slutsky equation, American Economic Review 42, S. 139.

Cowell, F.A., 1985, Tax evasion with labour income, Journal of Public Economics 26, S. $19-34$.

Cowell, F.A., 1987, The economic analysis of tax evasion, in: J. Hey und P. Lambert (Hrsg.), Surveys in the Economics of Uncertainty, London, S. 173 $-203$.

Cowell, F.A., 1989, Honesty is sometimes the best policy, European Economic Review 33, S. $605-617$.

Cowell, F.A. und J.P.F. Gordon, 1988, Unwillingness to pay - tax evasion and public good provision, Journal of Public Economics 36, S. 305 - 321.

Cross, R. und G.K. Shaw, 1982, On the economics of tax aversion, Public Finance 37, S. $36-47$.

Dardanoni, V., 1988, Optimal choices under uncertainty: the case of twoargument utility functions, Economic Journal 89, S. 429 - 450.

Deutsche Bundesbank, 1989, Geschäftsbericht der Deutschen Bundesbank für das Jahr 1988, Frankfurt.

Dreher E. und H. Tröndle, 1986, Strafgesetzbuch - Kurzkommentar, 43. Aufl., München.

Drèze, J.H. und F. Modigliani, 1972, Consumption decisions under uncertainty, Journal of Economic Theory 5, S. $308-335$.

Falkinger, J., 1988, Tax evasion and equity: a theoretical analysis, Public Finance 23, S. $388-395$. 
Feldmann, J. und J.A. Kay, 1981, Tax avoidance, in: P. Burrows und C.G. Veljanovski (Hrsg.), The Economic Approach to Law, London u.a.O., S. 320 $-333$.

Franzen, K., B. Gast und E. Samson, 1985, Steuerstrafrecht, München (3. Aufl.).

Frost, M., 1986, Steuerhinterziehung - eine modelltheoretische Analyse, Diplomarbeit, Hagen.

Gaertner, W. und A. Wenig (Hrsg.), 1985, The economics of the shadow economy, Berlin u.a.O.

Geiger, H., 1989, Die Abschaffung der Quellensteuer war dringend notwendig, Wirtschaftsdienst 69 , S. $332-334$.

Gordon, J.P.F., 1989, Individual morality and reputation costs as deterrents to tax evasion, European Economic Review 33, S. 797 - 805.

Gottlieb, D., 1985, Tax evasion and the prisoner's dilemma, Mathematical Social Sciences 10, S. $81-89$.

Graetz, M.J., 1980, Expenditure tax design, in: J.E. Pechmann (Hrsg.), What should be taxed: Income or Expenditure?, Washington, S. $161-276$.

Greenberg, J., 1984, Avoiding tax avoidance: a (repeated) game - theoretic approach, Journal of Economic Theory 32, S. 1 - 13.

Gutmann, P.M., 1977, The subterranean economy, Financial Analysts Journal, Nov./Dez., S. $26-27,34$.

Harrington, W., 1988, Enforcement leverage when penalties are restricted, Journal of Public Economics 37, S. 29 - 53.

Hatta, T. und R.J. Willke, 1982, Mosak's equality and the theory of duality, International Economic Review 23, S. 361 - 364.

Hagedorn, R., 1989, Witholding and non-withheld tax evasion: a comment, unveröffentlichtes Manuskript, Hagen.

Heyman, D.P. und M.J. Sobel, 1984, Stochastic models in operations research II - stochastic optimization, New York u.a.O.

Jakobsson, U., 1976, On the measurement of the degree of progression, Journal of Public Economics 5, S. $161-168$.

Kaldor, N., 1955, An expenditure tax, London. 
Kaldor, N., 1956, Indian tax reform, in: N. Kaldor, Reports on Taxation, Collected Economic Essays, Bd. 7, Duckworth und London, 1980, S. 31 $-108$.

Kay, J.A., 1980, The anatomy of tax avoidance, in: D. Collard, R. Lecomber und M. Slater (Hrsg.), Income Distribution: The Limits to Redestribution, Bristol, S. 135 - 149.

Keeney, R.L., 1973, Risk independence and multiattributed utility functions, Econometrica 41, S. 27 - 34.

Kelley, P.L., 1975, Is an expenditure tax feasibel, National Tax Journal 23, S. $237-253$.

Kohlas, J., 1877, Stochastische Methoden des Operations Research, Stuttgart.

Koskela, E., 1983, A note on progression, penalty schemes and tax evasion, Journal of Public Economics 22, S. 127 - 133.

Kottke, K., 1989, Was die Bank dem Fiskus mitteilen muB, Frankfurter Allgemeine Zeitung Nr. 123 vom 29.6.1989 (Blick durch die Wirtschaft), S. 7.

Krischausky, D., 1989, Besteuerung der Zinseinkünfte privater Haushalte, Dissertation rer. pol. Köln, Bergisch-Gladbach.

Kühn, R., H. Kutter und R. Hofmann, 1987, Abgabenordnung, Stuttgart (15. Aufl.).

Landsberger, M. und I. Meilijson, 1982, Incentive generating state dependent penalty system, Journal of Public Economics 19, S. 333 - 352.

Lodin, S.O., 1978, Progressive expenditure tax - an alternative?, Stockholm (1. Aufl. 1972).

Machina, M., 1989, Comparative statics and non-expected utility preferences, Journal of Economic Theory 47, S. $393-405$.

Marelli, M., 1984, On indirect tax evasion, Journal of Public Economics 25, S. $181-196$.

Marelli, M., 1987, The economic analysis of tax evasion: empirical aspects, in: J. Hey und P. Lambert (Hrsg.), Surveys in the Economics of Uncertainty, London, S. $204-228$.

Marelli, M. und R. Martina, 1988, Tax evasion and strategic behaviour of firms, Journal of Public Economics 37, S. 55 - 69.

Meade-Kommission, 1980, The structure and reform of direct taxation. Report of a commitee chaired by Professor J.E. Meade (Institute for Fiscal Studies), London u.a.O. 
Mieszkowski, P., 1980, The advisability and feasibility of an expenditure tax system, in: H.J. Aaron und M.J. Boskin (Hrsg.), The Economics of Taxation, Washington, S. $179-201$.

Mill, J.S., 1848, Principles of political economy, London (Nachdruck 1987, Fairfield).

Mitschke, J., 1980, Lebenseinkommensbesteuerung durch interperiodischen Progressionsausgleich, Steuer und Wirtschaft 2, S. $122-134$.

Mitschke, J., 1985, Steuer- und Transferordnung aus einem Guß: Entwurf und Neugestaltung der direkten Steuern und Sozialtransfers in der Bundesrepublik Deutschland, Baden-Baden.

Mossin, J., 1968, Taxation and risk-taking - an expected utility approach, Economica 35, S. $74-82$.

Mösbauer, H., 1989, Steuerstraf- und Steuerordnungswidrigkeitenrecht, München und Wien.

Nikaido, H., 1968, Convex structures and economic theory, New York.

Norton, D.A.G., 1988, On the economic theory of smuggling, Economica 55, S. $107-118$.

Peffekoven, R., 1980, Persönliche allgemeine Ausgabensteuer, Handwörterbuch der Finanzwissenschaft 2 , Tübingen (3. Aufl.), S. 417 - 452.

Pencavel, J.H., 1979, A note on income tax evasion, labor supply and nonlinear tax schedules, Journal of Public Economics 12, S. 115 - 124.

Pigou, A.C., 1928, A study in public finance, London (zitiert nach dem Nachdruck der 3. Aufl. von 1947 in 1962, London u.a.O.).

Pollak, H., 1989, Gestaltungs- und Folgeprobleme progressiver Ausgabensteuertarife, Referat auf dem Kongreß "Konsumorientierte Neuordnung des Steuersystems", Juni 1989, Heidelberg.

Poterba, J.M., 1987, Tax evasion and capital gains taxation, American Economic Review 77, P\&P, S. 234 - 239.

Pratt, J.W., 1964, Risk aversion in the small and in the large, Econometrica 32 , S. $122-136$.

Pyle, D.J., 1983, The economics of crime and law enforcement, London und Basingstoke.

Reinganum, J.F. und L.L. Wilde, 1986, Equilibrium verification and reporting policies in a model of tax compliance, International Economic Review 27, S. $739-760$. 
Rickard, J.A., A.M. Russel und T.D. Howroyd, 1982, A tax evasion model with allowance for retroactive penalties, Economic Record 58, S. 379 385 .

Rothschild, M. und J.E. Stiglitz, 1970, Increasing risk I: a definition, Journal of Economic Theory 2, S. 225 - 243.

Rothschild, M. und J.E. Stiglitz, 1971, Increasing risk II: its economic consequences, Journal of Economic Theory 3, S. 66 - 84.

Rubinstein, A., 1979, An optimal conviction policy for offenses that may have been committed by accident, in: S.J. Brams, A. Schotter und G. Schwödiauer (Hrsg.), Applied Game Theory, Würzburg und Wien, S. 406 - 413.

Rürup, B., 1989, Rücknahme der Quellensteuer - Eine Fehlentscheidung, Wirtschaftsdienst 69 , S. $334-337$.

Sandford, C.T., 1980, Tax compliance costs, evasion and avoidance, in:

D. Collard, R. Lecomber und M. Slater (Hrsg.), Income Distribution: The Limits to Redistribution, Bristol, S. 150 - 165.

Sandmo, A., 1969, Capital risk, consumption, and portfolio choice, Econometrica 37 , S. $586-599$.

Sandmo, A., 1974, Two-period models of consumption under uncertainty: A survey, in: J. Drèze (Hrsg.), Allocation under Uncertainty - Equilibrium and Optimality, London, S. $24-35$.

Sandmo, A., 1981, Income tax evasion, labour supply, and the equity-efficiency tradeoff, Journal of Public Economics 16, S. 265 - 288.

Sandmo, A., 1985, The effects of taxation on savings and risk-taking, in: A.J. Auerbach und M. Feldstein (Hrsg.), Handbook of Public Economics 1, Amsterdam, S. 265 - 311.

Schlicht, E., 1985, The shadow economy and morals: a note, in: W. Gaertner und A. Wenig (Hrsg.), The Economics of the Shadow Economy, Berlin u.a.O., S. $265-271$.

Schneider, D., 1987, Die Steuerreform und ihre Finanzierung in ihren Folgen für die Unternehmensfinanzierung mit Risikokapital, Der Betrieb 40, S. 2529 -2535 .

Scotchmer, S. und J. Slemrod, 1989, Randomness in tax enforcement, Journal of Public Economics 38, S. 17 - 32.

Schneeloch, D., 1986, Besteuerung und betriebliche Steuerpolitik, Bd. 1, München.

Schoemaker, P.J.H., 1982, The expected utility model: its variants, purposes, evidence and limitations, Journal of Economic Literature 20, S. 529 - 563. 
Seidel, C., 1989, Administration problems of an expenditure tax, Referat auf dem Kongreß "Konsumorientierte Neuordnung des Steuersystems", Juni 1989, Heidelberg.

Silberberg, E., 1978, The structure of economics - a mathematical analysis, New York u.a.O.

Shoup, C.S., 1969, Public finance, Chicago.

Skinner, J. und J. Slemrod, 1985, An economic perspective on tax evasion, National Tax Journal 38, S. 345 - 353.

Slemrod, J., 1985, An empirical test for tax evasion, The Review of Economics and Statistics 57, S. $232-238$.

Slemrod, J. und S. Yitzhaki, 1987, The optimal size of a tax collection agency, Scandinavian Journal of Economics 89, S. 183 - 192.

Spicer, M.W. und S.B. Lundstedt, 1976, Understanding tax evasion, Public Finance 31, S. 295 - 305.

Srinivasan, T.N., 1973, Tax evasion: a model, Journal of Public Economics 2, S. $339-346$.

Statistisches Bundesamt, 1989, Statistisches Jahrbuch für die Bundesrepublik Deutschland 1989, Wiesbaden.

Stiglitz, J.E., 1969, The effects of income, wealth and capital gains taxation on risk-taking, Quaterly Journal of Economics 83, S. 262 - 283.

Stiglitz, J.E., 1983, Some aspects of the taxation of capital gains, Journal of Public Economics 21, S. 257 - 294.

Stiglitz, J.E., 1986, Economics of the public sector, New York und London.

Strange, S., 1986, Casino capitalism, London.

Streck, M., 1986, Die Steuerfahndung, Köln.

Thirlwall, A.P., 1987, Nicholas Kaldor, Brighton.

Tillmann, G., 1990, Zur Belastungswirkung von Quellensteuer und Kontrollmitteilungen auf unterschiedliche Einkommensgruppen, Finanzarchiv (erscheint demnächst).

Tipke, K. und H.W. Kruse, 1988, Abgabenordnung-Kommentar, 12. Aufl., Köln.

Tipke, K., 1989, Lehren aus der Steuerreform, Steuer und Wirtschaft N.F. 19, S. $291-310$.

Tullock, G., 1974, Does punishment deter crime?, Public Interest 36, S. 103 111. 
Varian, H., 1984, Microeconomic analysis, New York und London (2. Aufl.).

Virmani, A., 1989, Indirect tax evasion and production efficiency, Journal of Public Economics 39, S. 223 - 237.

Walter, I., 1985, Secret money - the world of international financial secrecy, London.

Watson, H., 1985, Tax evasion and labor markets, Journal of Public Economics 27, S. $231-246$.

Weiss, L., 1976, The desirability of cheating incentives and randomness in the optimal income tax, Journal of Political Economy 84, S. 1343 - 1352.

Wenger, E., 1985, Lebenszeitbezogene Gleichmäßigkeit als Leitidee der Abschnittsbesteuerung, Finanzarchiv N.F. 43, S. 307 - 327.

Wenig, A., 1988, The shadow economy in the Federal Republic of Germany, Commission of the European Communities, Directorate General of Employment, Social Affairs, and Education (Hrsg.), V/1784/88 - EN, Brüssel.

Wiegard, W., 1984, Schwarzarbeit und Besteuerung, in: W. Schäfer (Hrsg.), Schattenökonomie - Theoretische Grundlagen und wirtschaftspolitische Konsequenzen, Göttingen, S. 122 - 156.

Wiegard, W., 1987, Was brachte - oder bringt - die Optimalsteuertheorie?, in: B. Rahmann und O. Roloff (Hrsg.), Beschäftigungspolitik zwischen Abgabenwiderstand und Ausgabenwachstum, Regensburg, S. 99 - 137.

Yaari, M.E., 1969, Some remarks on measures of risk aversion and on their uses, Journal of Economic Theory 1, S. $315-329$.

Yaniv, G., 1988, Withholding and non-withheld tax evasion, Journal of Public Economics 35, S. 183 - 204.

Yitzhaki, S., 1974, A note on 'Income tax evasion - a theoretical analysis', Journal of Public Economics 3, S. 201 - 202.

Yitzhaki, S., 1987, On the excess burden of tax evasion, Public Finance Quarterly 15 , S. $123-137$. 


\section{FINANZWISENSCHAFTLICHE SCHRIFTEN}

Band 1 Werner Steden: Finanzpolitik und Einkommensverteilung. Ein Wachstums- und Konjunkturmodell der Bundesrepublik Deutschland. 1979.

Band 2 Rainer Hagemann: Kommunale Finanzplanung im foderativen Staat. 1976.

Band 3 Klaus Scherer: Maßstäbe zur Beurteilung von konjunkturellen Wirkungen des offentlichen Haushalts. 1977.

Band 4 Brita Steinbach: "Formula Flexibility" - Kritische Analyse und Vergleich mit diskretionârer Konjunkturpolitik. 1977.

Band 5 Hans-Georg Petersen: Personelle Einkommensbesteuenung und Inflation. Elne theoretisch-empirische Analyse der Lohn- und veranlagten Einkommensteuer in der Bundesrepublik Deutschland. 1977.

Band 6 Friedemann Tetsch: Raumwirkungen des Finanzsystems der Bundesrepublik Deutschland. Eine Untersuchung der Auswirkungen der Finanzreform von 1969 auf die Einnahmenposition der untergeordneten Gebletskorperschaften und ihrer regionalpolitischen Zieladăquanz. 1978.

Band 7 Wilhelm Pfahler: Normative Theorie der fiskalischen Besteuerung. Ein methodologischer und theoretischer Beitrag zur Integration der normativen Besteuenungstheorie in der Wohlfahrtstheorie. 1978.

Band 8 Wolfgang Wiegard: Optimale Schattenpreise und Produktionsprogramme fur offentliche Unternehmen. Second-Best Modelle im finanzwirtschaftlichen Staatsbereich. 1978.

Band 9 Hans P. Fischer: Die Finanzierung des Umweltschutzes im Rahmen einer rationalen Umweltpolitik. 1978.

Band 10 Rainer Paulenz: Der Einsatz finanzpolitischer Instrumente in der Forschungs- und Entwicklungspolitik. 1978.

Band 11 Hans-Joachim Hauser: Verteilungswirkungen der Staatsverschuldung. Eine kreislauftheoretische Inzidenzbetrachtung. 1979.

Band 12 Gunnar Schwarting: Kommunale Investitionen. Theoretische und empirische Untersuchungen der Bestimmungsgründe kommunaler Investitionstâtigkeit in NordrheinWestfalen 1965-1972. 1979.

Band 13 Hans-Joachim Conrad: Stadt-Umland-Wanderung und Finanzwirtschaft der Kernstadte. Amerikanische Erfahrungen, grundsătzliche Zusammenhânge und eine Fallstudie für das Ballungsgebiet Frankfurt am Main. 1980.

Band 14 Cay Folkers: Vermógensverteilung und staatliche Aktivităt. Zur Theorie distributiver Prozesse im Interventionsstaat. 1981.

Band 15 Helmut Fischer: US-amerikanische Exportförderung durch die DISC-Gesetzgebung. 1981.

Band 16 Günter Ott: Einkommensumverteilungen in der gesetzlichen Krankenversicherung. Eine quantitative Analyse. 1981.

Band 17 Johann Hermann von Oehsen: Optimale Besteuerung. (Optimal Taxation). 1982.

Band 18 Richard Kossler: Sozialversicherungsprinzip und Staatszuschússe in der gesetzlichen Rentenversicherung. 1982.

Band 19 Hinrich Steffen: Zum Handlungs- und Entscheidungssplelraum der kommunalen Investitionspolitik in der Bundesrepublik Deutschland. 1983.

Band 20 Manfred Scheuer: Wirkungen einer Auslandeverschuldung des Staates bei flexiblen Wechselkursen. 1983.

Band 21 Christian Schiller: Staatsausgaben und crowding-out-Effekte. Zur Effizienz einer Finanzpolitik keynesianischer Provenienz. 1983.

Band 22 Hannelore Weck: Schattenwirtschaft: Elne Moglichkelt zur Einschrânkung der offentlichen Verwaltung? Eine ökonomische Analyse. 1983. 
Band 23 Wolfgang Schmitt: Steuern als Mittel der Einkommenspolitik. Eine Ergânzung der Stabilitătspolitik? 1984.

Band 24 Wolfgang Laux: Erhōhung staatswirtschaftlicher Effizienz durch budgetăre Selbstbeschrânkung? Zur Idee einer verfassungsmaßßig verankerten Ausgabengrenze. 1984.

Band 25 Brita Steinbach-van der Veen: Steuerinzidenz. Methodologische Grundlagen und empirisch-statistische Probleme von Landerstudien. 1985.

Band 26 Albert Peters: Okonomische Kriterien für eine Aufgabenverteilung in der Marktwirtschaft. Eine deskriptive und normative Betrachtung für den Allokationsbereich. 1985.

Band 27 Achim Zeidler: Möglichkeiten zur Fortsetzung der Gemeindefinanzreform. Eine theoretische und empirische Analyse. 1985.

Band 28 Peter Bartsch: Zur Theorie der lângerfristigen Wirkungen 'expansiver' Fiskalpolitik. Eine dynamische Analyse unter besonderer Berücksichtigung der staatlichen Budgetbeschrânkung und ausgewăhlter Möglichkeiten der ôffentlichen Defizittinanzierung. 1986.

Band 29 Konrad Beiwinkel: Wehrgerechtigkeit als finanzpolitisches Verteilungsproblem. Móglichkeiten einer Kompensation von Wehrungerechtigkeit durch monetäre Transfers. 1986.

Band 30 Wolfgang Kitterer: Effizienz- und Verteilungswirkungen des Steuersystems. 1986.

Band 31 Heinz Dieter Hessler: Theorie und Politik der Personalsteuern. Eine Kritik ihrer Einkommens- und Vermogensbegriffe. 1987.

Band 32 Wolfgang Scherf: Die beschăftigungspolitische und fiskalische Problematik der Arbeitgeberbeitrăge zur Rentenversicherung. Eine Auseinandersetzung mit der Kritik an der lohnbezogenen Beitragsbemessung. 1987.

Band 33 Andreas Măstle: Die Steuerunion. Probleme der Harmonisienung spezifischer Gütersteuern. 1987.

Band 34 Gônter Ott: Internationale Verteilungswirkungen im Finanzausgleich der Europäischen Gemeinschaften. 1987.

Band 35 Heinz Haller: Zur Frage der zweckmaßioen Gestalt gemeindlicher Steuern. Ein Diskussionsbeitrag zur Gemeindesteverreform. 1987.

Band 36 Thomas Kuhn: Schlüsselzuweisungen und fiskalische Ungleichheit. Eine theoretische Analyse der Verteilung von Schlússelzuweisungen an Kommunen. 1988.

Band 37 Walter Hahn: Steuerpolitische Willensbildungsprozesse in der Europäischen Gemeinschaft. Das Beispiel der Umsatzssteuer-Harmonisienung. 1988.

Band 38 Ulrike Hardt: Kommunale Finanzkraft. Die Problematlk oiner objektiven Bestimmung kommunaler Einnahmemóglichkelten in der gemeindlichen Haushaltsplanung und im kommunalen Finanzausgleich. 1988.

Band 39 Jochen Michaelis: Optimale Finanzpolitik im Modell Oberlappender Generationen. 1989.

Band 40 Bernd Raffelhüschen: Anreizwirkungen der sozialen Alterssicherung. Eine dynamische Simulationsanalyse. 1989.

Band 41 Berend Diekmann: Die Anleihe- und Darlehenstransaktionen der Europäischen Gemeinschaften. 1990.

Band 42 Helmut Kaiser: Konsumnachfrage, Arbeitsangebot und optimale Haushaltsbesteuerung. Theoretische Ergebnisse und mikrookonometrische Simulation für die Bundesrepublik Deutschland. 1990.

Band 43 Rüdiger von Kleist: Das Gramm-Rudman-Hollings-Gesetz. Ein gescheiterter Versuch der Haushaltskonsolidierung. 1991.

Band 44 Rolf Hagedorn: Steuerhinterziehung und Finanzpolitik. Ein theoretischer Beitrag unter besonderer Beruckssichtigung der Hinterzlehung von Zinsertragen. 1991. 


\section{Christian Suhr}

\section{Rechtsgut der Steuerhinterziehung und Steuerverkürzung im Festsetzungsverfahren}

Frankfurt/M., Bern, New York, Paris, 1989. XII, 193 S.

Europäische Hochschulschriften: Reihe 2, Rechtswissenschaft.

Bd. 868

ISBN 3-631-42110-9

br./lam. DM 55.--/sFr. 50.--

Der Erfolg der Steuerhinterziehung, die Steuerverkürzung, bereitet in Theorie und Praxis erhebliche Schwierigkeiten. Bedeutsame Einzelfragen, wie die Einordnung des "Kompensationsverbots", die Abgrenzung der Hinterziehung auf Zeit und auf Dauer, die Beurteilung der Vermögensteuerhinterziehung, die Berechnung von Hinterziehungszinsen sowie die Bestimmung des Tatobjekts sind umstritten. Der Verfasser entwickelt für diese und weitere Fragen ein einheitliches Lösungskonzept, das von einer detaillierten Untersuchung des Schutzgegenstands (= Rechtsgut) der Steuerhinterziehung ausgeht und zahlreiche Parallelen zu den Vermögensdelikten des Kernstrafrechts zieht.

Aus dem Inhalt: Rechtsgut, Tatobjekt und Erfolgsunrecht der Steuerhinterziehung - Steuerhinterziehung auf Zeit und auf Dauer - Kompensationsverbot - Hinterziehung von Vermögensteuer - Hinterziehungszinsen

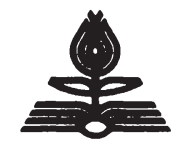

Verlag Peter Lang Frankfurt a.M. Bern $\cdot$ New York $\cdot$ Paris Auslieferung: Verlag Peter Lang AG, Jupiterstr. 15, CH-3000 Bern 15 Telefon (004131) 321122, Telex pela ch 912 651, Telefax (004131) 321131 
Rolf Hagedorn - 978-3-631-75230-2

Downloaded from PubFactory at 01/11/2019 06:51:51AM

via free access 
Rolf Hagedorn - 978-3-631-75230-2

Downloaded from PubFactory at 01/11/2019 06:51:51AM

via free access 
Rolf Hagedorn - 978-3-631-75230-2

Downloaded from PubFactory at 01/11/2019 06:51:51AM

via free access 